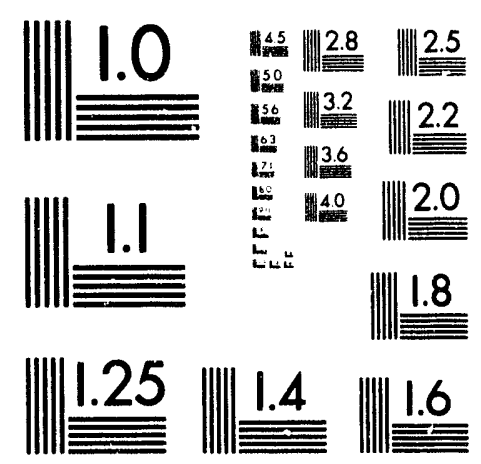



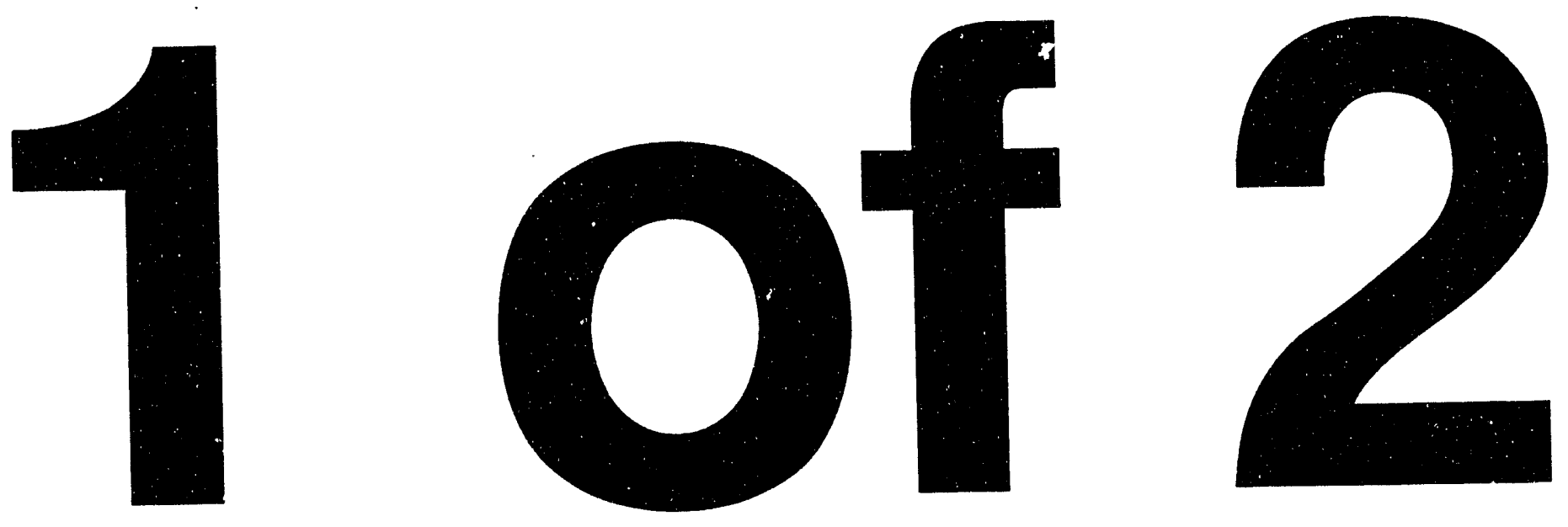


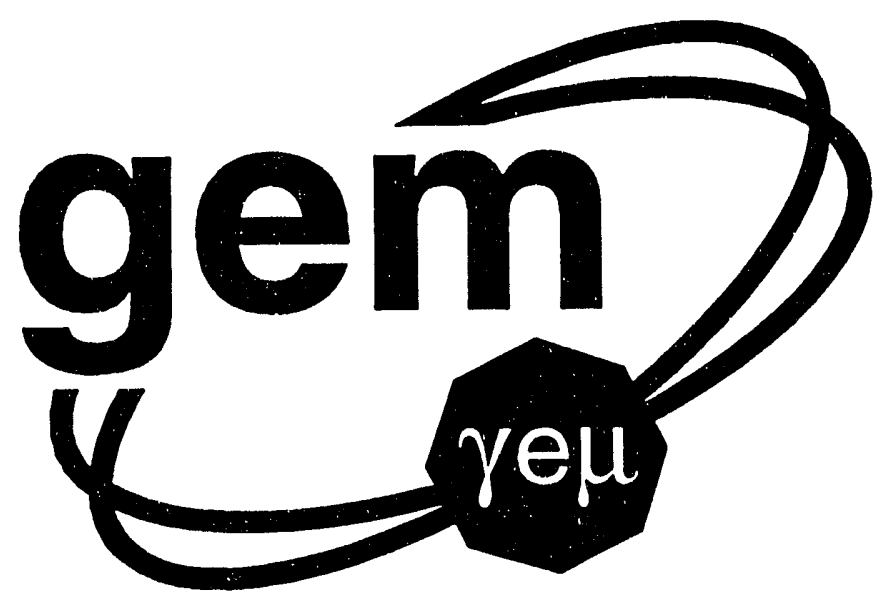

\section{The RDT-RPC Technology Option for GEM}

Boston University, Brown University, C.S. Draper Laboratories, Indiana University, Institute for Theoretical and Experimental Physics - (ITEP) Moscow, Joint Institute for Nuclear Research, Lawrence Livermore National Laboratory, LeCroy Corporation, Louisiana State University, Massachusetts Institute of Technology, Michigan State University, Moscow State University, Superconducting Super Collider Laboratory, University of Rome \& Instituto Nazionale di Fisica Nucleare, University of Tennessee

January 29, 1993

Abstract:

A muon system for the GEM SSCL detector based on Round Drift Tubes (RDT) has many attractive features. The Pt-trigger and beam crossing tag for the RDT tracking system can be accomplished by either a PWC system, similar but less costly in design than Cathode Strip Chambers, or by a promising new type of RPC chamber. Given the economic construction of these chamber technologies a system can be envisioned with both good momentum reconstruction performance and pattern recognition capabilities. Fullscale chamber prototypes based on the RDT and RPC technologies have been constructed and tested successfully at the Texas Test Rig. Spatial resolutions have been measured in two designs of RDT chambers which meet or exceed the baseline resolution requirement of $100 \mu \mathrm{m}$ per chamber layer. 


\title{
The RDT-RPC Technology Option for GEM
}

\author{
Boston University, Brown University, Draper Laboratories, Indiana University, \\ ITEP-Moscow, JINR-Dubna, Lawrence Livermore National Laboratory, \\ LeCroy Corporation, Louisianna State University, \\ Massachusetts Institute of Technology, Michigan State University, \\ Moscow State University, Superconducting Super Collider Laboratory, \\ University of Rome \& Instituto Nazionale di Fisica Nucleare, \\ University of Tennessee
}

January 29, 1993

\section{Abstract:}

A muon system for the GEM SSCL detector based on Round Drift Tubes (RDT) has many attractive features. The Pt-trigger and beam crossing tag for the RDT tracking system can be accomplished by either a PWC system, similar but less costly in design than Cathode Strip Chambers, or by a promising new type of RPC chamber. Given the economic construction of these chamber technologies a system can be envisioned with both good momentum reconstruction performance and pattern recognition capabilities. Full-scale chamber prototypes based on the RDT and RPC technologies have been constructed and tested successfully at the Texas Test Rig. Spatial resolutions have been measured in two designs of RDT chambers which meet or exceed the baseline resolution requirement of $100 \mu \mathrm{m}$ per chamber layer.

\section{DISCLAIMER}

\footnotetext{
This report was prepared as an account of work sponsored by an agency of the United States Government. Neither the United States Gnvernment nor any agency thereof, nor any of their employees, makes any warranty, express or implied, or assumes any legal liability or responsibility for the accuracy, completeness, or usefulness of any information, apparatus, product, or process disclosed, or represents that its use would not infringe privately owned rights. Reference herein to any specific commercial product, process, or service by trade name, trademark, manufacturer, or otherwise does not necessarily constitute or imply its endorsement, recommendation, or favoring by the United States Government or any agency thereof. The views and opinions of authors expressed herein do not necessarily state or reflect those of the United States Government or any agency thereof.
} 


\section{Table of Contents}

(1) Introduction ...................................................................................................... 3

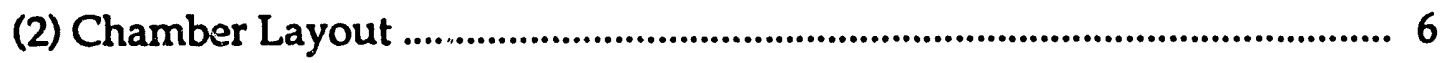

(3) System Parameters ……................................................................................... 17

(4.0) Design of the Round Drift Tube Chambers .............................................. 23

(4.1) RDT Stereo

(5) RPC Design .......................................................................................... 43

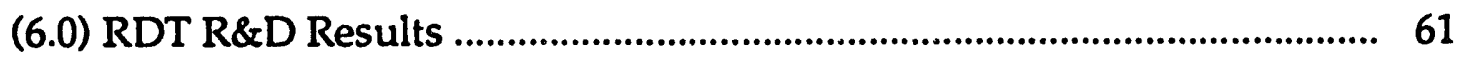

(6.1) Factors Affecting Precision .......................................................................... 99

(6.2) RPC R\&D Results............................................................................... 116

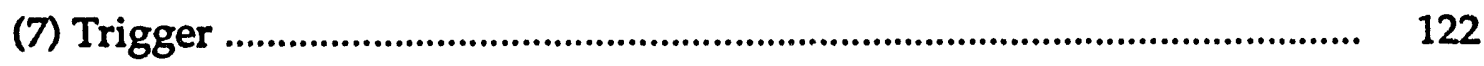

(8) System Performance ................................................................................. 145

(8.1) Trigger Performance .................................................................................. 145

(8.2) Momentum Reconstruction ......................................................................... 149

Appendix A: RPC Construction and Costs .................................................. 159

Appendix B: Details of Accidental Calculation ............................................... 206

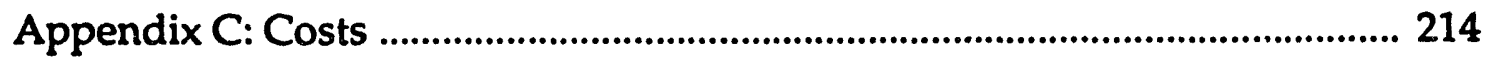

Appendix D: RDT System with a PWC ToTrigger ........................................ 216

Appendix E: RDT Stand-alone Trigger ........................................................... 221 


\section{(1.0) Introduction:}

The muon system described in this report is based on RDTs for precision tracking and RPCs (or PWCs) for triggering. Drift tubes, $2.54 \mathrm{~cm}$ in diameter, are configured in each of three superlayers with a multiplicity of 8:10:8. Pairs of anode wires of the even (odd) tubes of a given layer in a superlayer are tied together through a delay line. The time difference yields a correlated $X-Y$ measurement. The $Y$ resolution (along the wire) by this method is about $10 \mathrm{~cm}$ RMS. In each superlayer there will be $2 \mathrm{U}$ and $2 \mathrm{Y}$ chamber planes oriented in small angle $(4 \mathrm{mr})$ stereo. The other layers in a superlayer will have either 4 or 6 axial tubes [parallel (perpendicular) to the axis of the solenoid for barrel (endcaps)]. The small-angle stereo layers in a superlayer will contribute almost equally to the momentum resolution as the axial layers, while also providing redundant non-bend plane coordinate measurements. It is proposed that both the barrel and endcaps have this 8:10:8 small-angle stereo configuration.

The trigger of the RDT system would be either Resistive Plate Counters (RPCs) or Proportional Wire Chambers (PWCs). The RPC solution was adapted at the onset of the GEM R\&D Program because of its many attractive features. At this time the RPC technology has not demonstrated the rate performance needed for application in the endcaps at $\mathrm{L}=10^{33} / \mathrm{cm}^{2} \mathrm{sec}$ given the uncertainties of the neutron fluence there. However the RDT technology has sufficient strong points not to discard it completely as a concept, and to propose a substitute for the RPCs. In fact one can envision a conservative muon system based on RDT tracking with a PWCs acting as a trigger. Preliminary estimates indicate that such a system would be both inexpensive, straightforward, and have the appropriate performance characteristics.

To maintain a simplicity of design, all the RDT and RPC (PWC) chambers in the proposed detector will be rectangular. Perhaps surprisingly, rectangular chambers, which are a natural deployment for the barrel region, also offer several attractive features for the endcaps and none of putative drawbacks there. First, we find that the momentum and trigger resolutions meet the baseline specification in the endcaps with this rectangular chamber design. Second, roughly $1 / 3$ of the chamber area is overlapped and hence a muon penetrating this region will traverse twice the usual number of tubes where the resolution at high momentum and pattern recognition will be improved and the low momentum resolution not significantly degraded. Third, the alignment paths in both the barrel and endcaps are located through small cracks between chamber planes allowing chambers within a sector as well as chambers of different sectors to be tied together while maintaining almost full solid angle coverage.

The trigger function will be performed with three layers of RPCs mounted in each of the superlayers. (PWCs could also be deployed for this purpose.) In a trigger system based on RPCs each chamber in a superlayer will furnish a bend plane $(X)$ and nonbend plane $(Y)$ signal for the Level 1 trigger. There would be three chambers per superlayer. A 2/3 coincidence requirement of the RPC layers in each superlayer will 
be imposed to yield an efficient trigger. All three superlayers of RPCs are in turn placed in coincidence. A similar system could be envisioned based on PWCs.

The following is a list of advantages of the technologies proposed:

\section{RDT Features for Momentum Reconstruction:}

- Proven technology - used by several groups at SLAC, CDF, E706-FNAL, AMY-TRISTAN, and several groups at CERN.

- Can be operated in either the proportional or limited streamer mode.

- Can be pressurized to improve resolution: $50 \mu \mathrm{m}$ has been obtained at $4.5 \mathrm{~atm}$.

- Full-scale prototypes have been constructed by the GEM Muon group which have demonstrated the baseline resolution.

- $\quad$ RDTs can be used to trigger the muon system - adding redundancy to the system in a stand-alone mode or with an external T0 signal.

- A RDT chamber forms a laminated tube structure which is both rigid and light weight. Hence the chamber need be supported only at its ends, making the support structure and alignment path layout practical.

- Layer resolution independent of the angle of incidence.

- Inherent cylindrical symmetry makes Lorentz angle correction straightforward.

\section{Trigger:}

\section{Based on RPC}

- Intrinsically fast device - measured jitter at low rates $<2 \mathrm{~ns}$.

- $\quad$ Easy to construct - light weight laminated structure with both $X$ and $Y$ readout possible for each chamber gap.

- $\quad$ Large pulses - typically $0.5 \mathrm{~V}$ in $50 \Omega$.

- $X-Y$ readout for each chamber gap.

Based on PWC

- Principle of operation can be modeled so that characteristics can be designed. 
- A narrow gap chamber ( $2.5 \mathrm{~mm}$ wire spacing) will be intrinsically fast.

- Can be instrumented to give T0 from the anode wires, and a bend plane measurement for a Pt-dependent signal. (CSC design without precision.)

The muon chambers of the GEM detector will have to operate in a hostile environment. Not only must the system discriminate against low momentum charged particles, but also operate gracefully in a large fluence of neutrons. For these reasons the pattern recognition and rate capabilities of the system are as important as the single track momentum resolution. A priori, the muon system must be redundant - having as many space points and cross check as is economically feasible.

The following table lists the information provided by this system.

Table 1.1

Information per Incident Muon Track

Bend plane Non-bend plane

\begin{tabular}{|c|c|c|c|c|c|}
\hline & Trigger & Tracking & Trigger & $\begin{array}{l}\text { Tracking } \\
\text { time }\end{array}$ & stereo \\
\hline \multicolumn{6}{|c|}{ 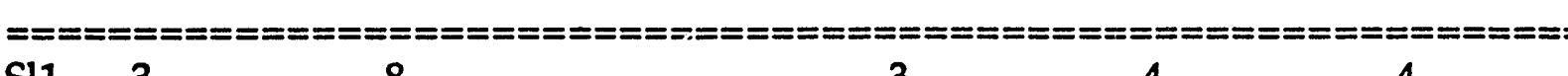 } \\
\hline $\mathrm{S} 12$ & 3 & 10 & 3 & 6 & 4 \\
\hline $\mathrm{S} 13$ & 3 & 8 & 3 & 4 & 4 \\
\hline
\end{tabular}

We note that there are a total of 35 measurement points in each of the bend and nonbend planes for a muon track. Such a high redundancy will be quite useful in making the proposed system operational at the expected high background to signal environment. 


\section{(2.0) Chamber Layout}

A sectional view of the RDT chambers in a quadrant of the GEM detector is shown in Fig. 2.1. Both the barrel and endcap regions are divided into 16 equal segments in the $\phi$ dimension. These segments are subdivided into projective towers consisting of 3 superlayers of $8+10+8$ planes. Each superlayer has 4 layers of small angle stereo tubes $(2 U+2 V)$ in addition to the $4+6+4$ axial tubes.

In the barrel region, 3 chambers are required to cover the half-length in the middle and outer superlayers, while two chambers are sufficient in the inner superlayer. The cracks associated with these divisions are arranged to accommodate the projective alignment scheme and to maximize the acceptance high quality tracks. An axial view of a barrel quadrant is shown in Fig. 2.2. To allow penetration for support structure and alignment paths, the middle superlayer in the barrel consist of 6 chambers that are alternately long-narrow or short-wide as shown in Fig. 2.3. The details of the $\phi$ and $\theta$ sections are shown in Figs. 2.4 and 2.5, respectively. The barrel layers overlap in the $\phi$ dimension due to the beveled chamber edges, as shown in the drawing labeled Section A. Section D shows the crack in $\theta$ due to the chamber end plate and plugs $(5 \mathrm{~cm})$, plus a gap of $5 \mathrm{~cm}$ for cables, connectors, support structure, etc. Sections $B$ and $C$ show the larger gaps that exist only at the chamber corners for the alignment paths. The number, channel count, location, size and weight of the chambers in the barrel regions are specified in Table 2.1.

In principle there can be an alignment path at each corner of each of the chambers in the barrel, a total of 24 paths per segment. However, we believe that it should be possible to design a fixture which would tie together the corners of adjacent chambers within a segment. This would allow these corners to be referenced by a single alignment path and reduce the number of paths to 12 per segment.

In the endcap regions, there are two projective sections in the $\theta$ dimension. Since there is a larger radial separation in this region, the chambers are allowed to overlap in both $\theta$ and $\phi$. This permits the use of rectangular chambers, identical in design to the barrel chambers, as shown in Fig. 2.6. Rectangular chambers in the endcap segments have two other advantages. Alignment paths along the $16^{\circ}$ projection can be easily accommodated in triangular shaped cracks that exist where four chambers meet. The chambers also have a substantial overlap area that will help with local and global alignment. There are a total of 9 alignment paths per endcap segment. Table 2.2 specifies the number, channel count, location, size and weight of the chambers in the endcaps.

The RPC (or PWC) layout exactly matches the RDT chambers. Since the RPC's are expected to be light and flexible, they could be attached to the surface of the RDT's. The estimated acceptance of the RDT/RPC system is listed in Table 2.3. The chamber ends and alignment paths add up to a $6 \%$ loss of solid angle which is equal to .09 
units of $\eta$ (excluding the region which is adjacent to the inaccessible forward direction). 


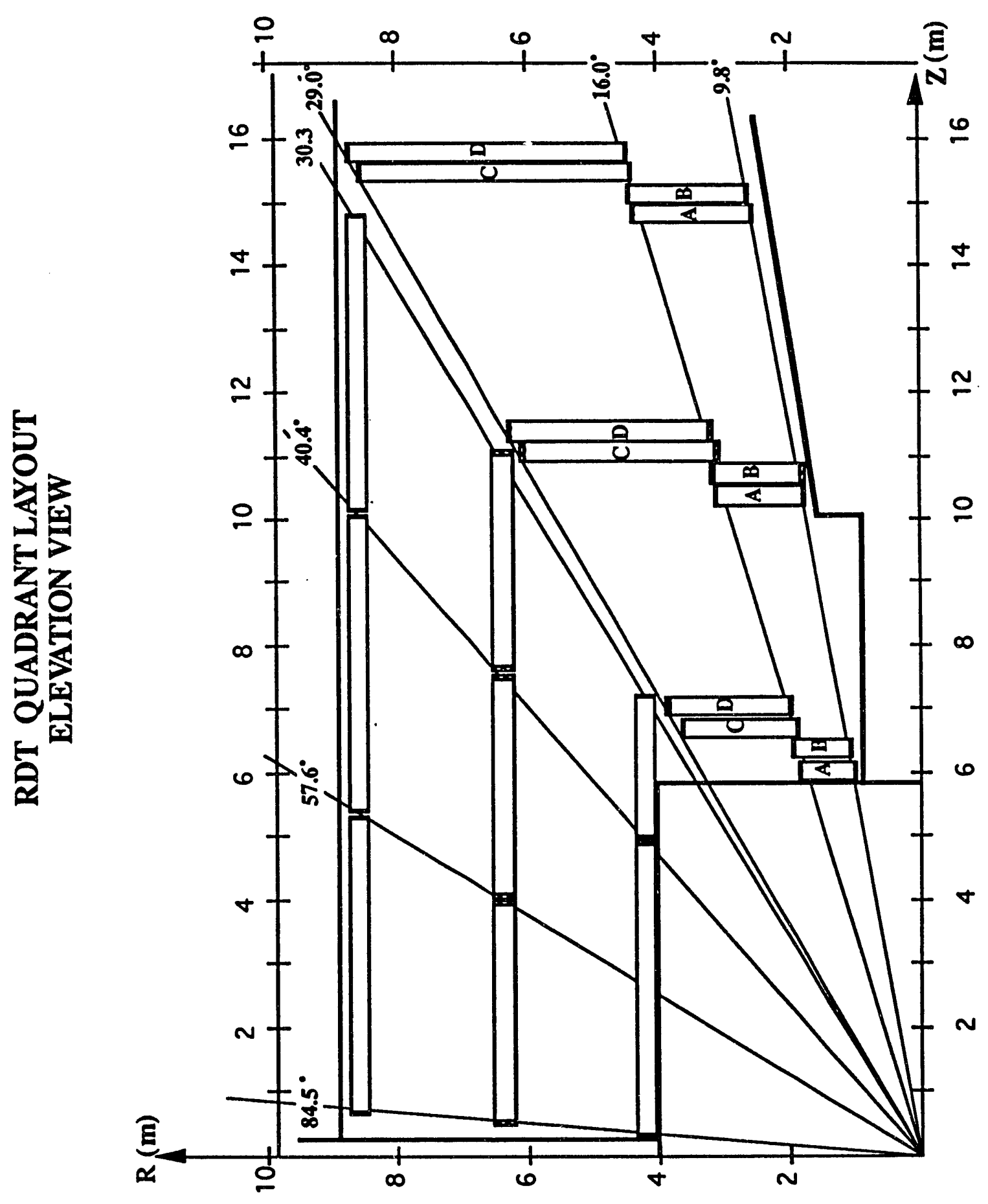

Fig. 2.1 


\section{RDT BARREL QUADRANT AXIAL VIEW}

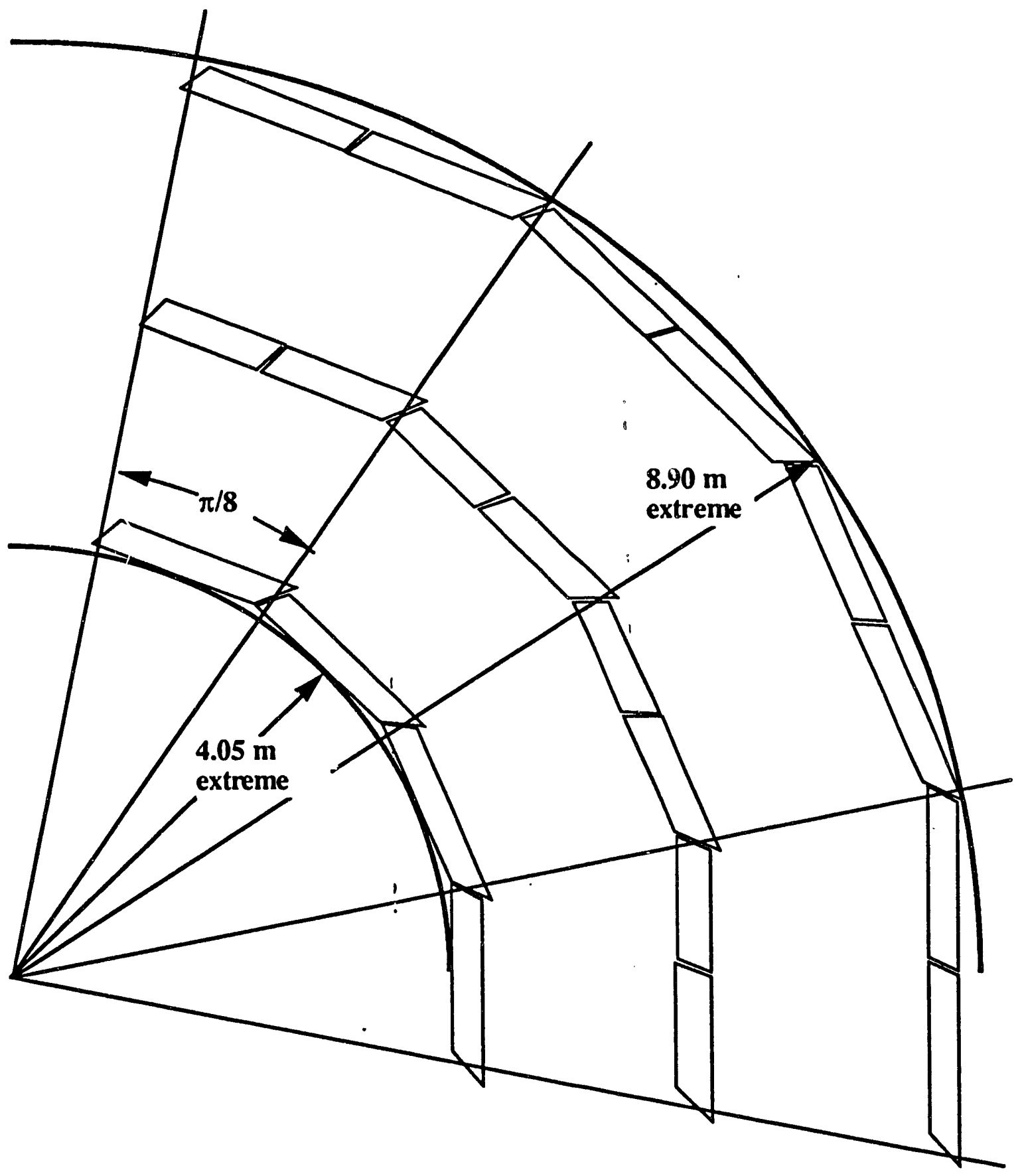




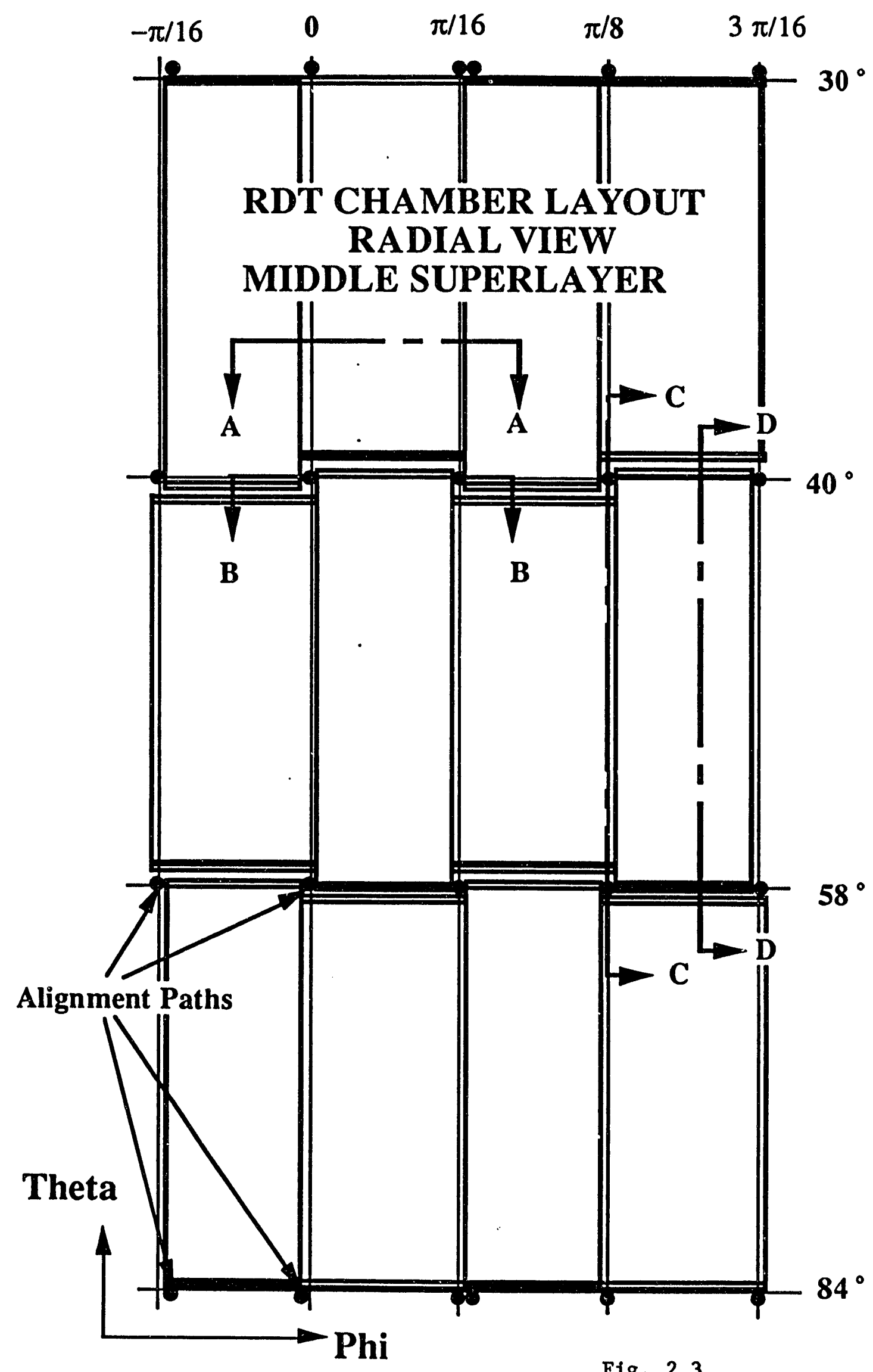

Fig. 2.3 


\section{RDT BARREL, MIDDLE SUPERLAYER PHI OVERLAP DETAIL}

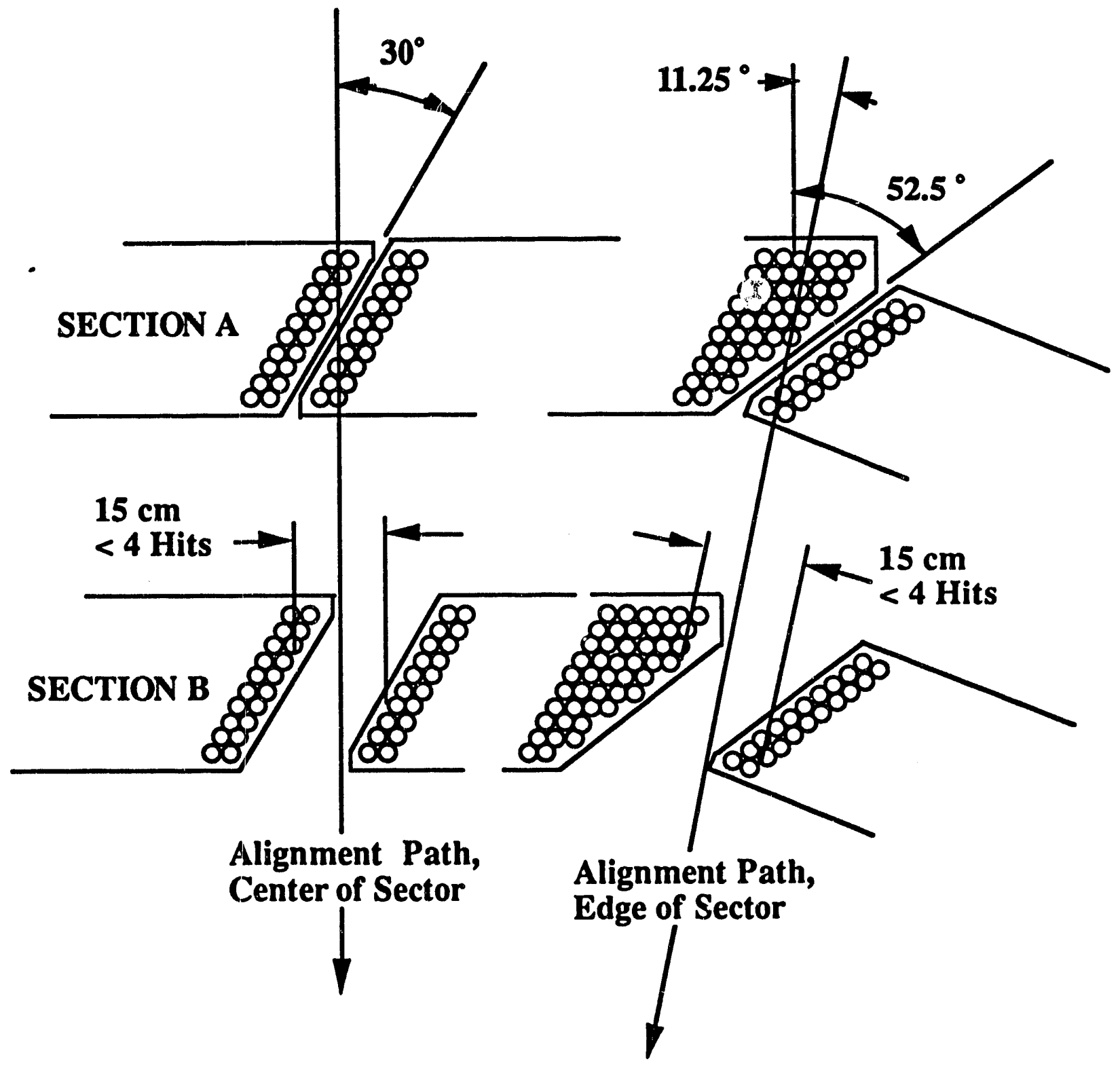

INTERACTION POINT

Fig. 2.4 


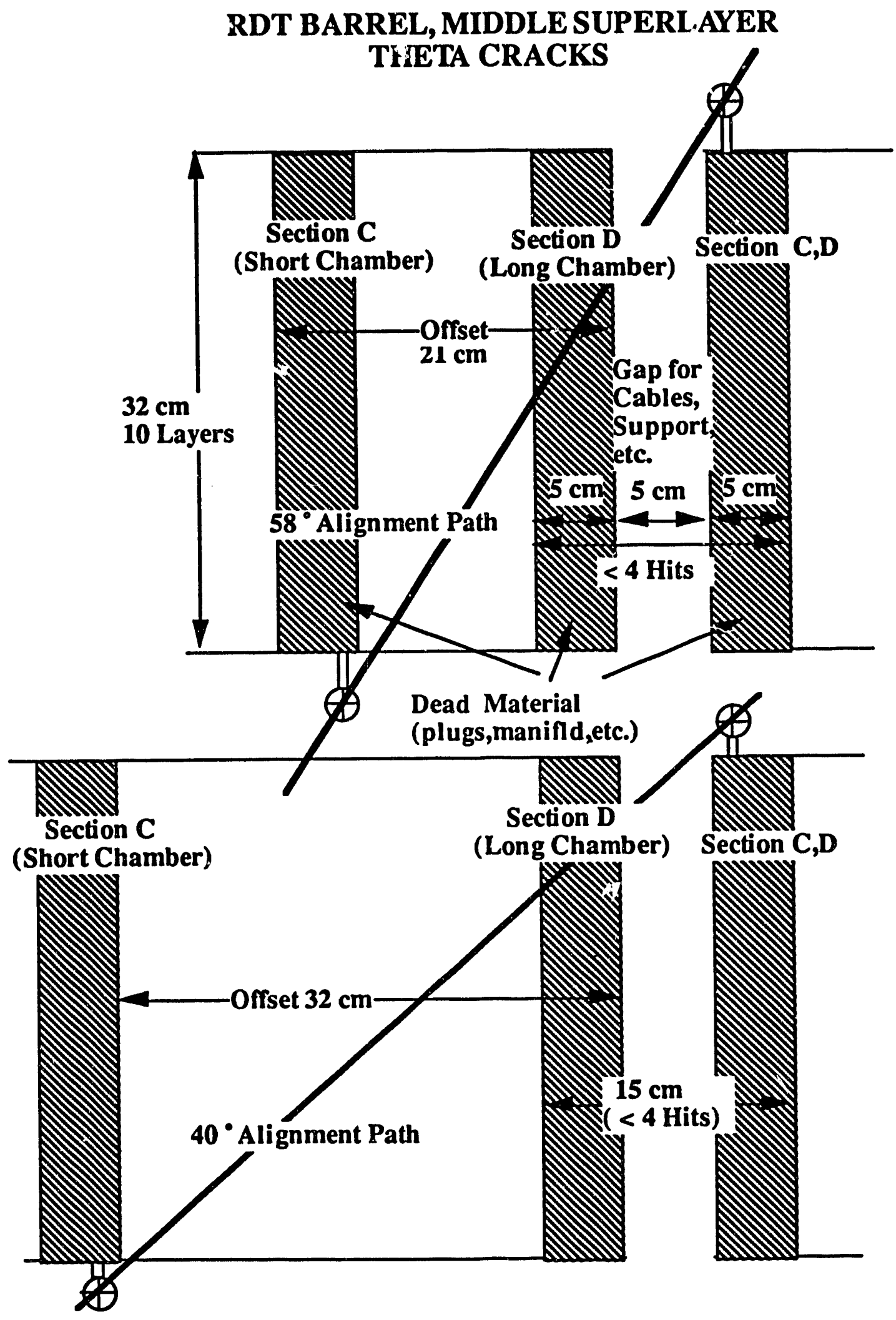

Fig. 2.5 


\section{GEM BARREL RDT CHAMBER SPECS 16 SECTORS; 8+10+8; 25 mm Dia.}

INPUT PARAMETERS:

Theta angular coverage :

Tube Material, density:

Tube Wall: (mm)

$\begin{array}{rr}84.5 & 30.3 \\ \text { Alum. } & 2.7 \\ 0.30 & \\ 19.05 & 2.7 \\ 6.40 & 0.25 \\ 16 & \end{array}$

Plate Thickness (mm), density:

Case Thickness (mm), density:

\# of Segments:

2.7

2.7

0.25

VARIABLES:

Radius (m):

Chambers/Theta

Chambers/segmnt

\# of Layers:

Tube Dia: (mm)

4.2

2

12

10

$25.4 \quad 25.4$

8.6 TOTAL

3

2

8

26

25.4

RESULTS:

Tube Length: (m)

\section{4}

512

Tubes/Chamber:

Avg. width: (m)

1.67

Chamb. thick.: (m)

0.28

Chambers/Half:

Chambers Total

Tubes/Half:

32

64

Tubes Total

16384

32768

3.4

496

4.6

528

1.26

1.69

0.32

96

0.28

192

47616

95232

96

192

50688

101376

$209 \mathrm{~km}$

Weight/chamber (lb)

Tubes:

247

Manifold plates:

Plugs:

Case:

Electronics,cable:

Total/Chamber:

Weight/Segment:

Weight/Half:

Weight Total
105

57

50

256

715

1429

22867

45733
243

92

55

41

248

679

4075

65208

130415
85 ton

169 ton
348

106

59

68

264

844

5066

81051

162102
224

448

114688

229376 


\section{RDT ENDCAP LAYOUT PROJECTIVE VIEW}

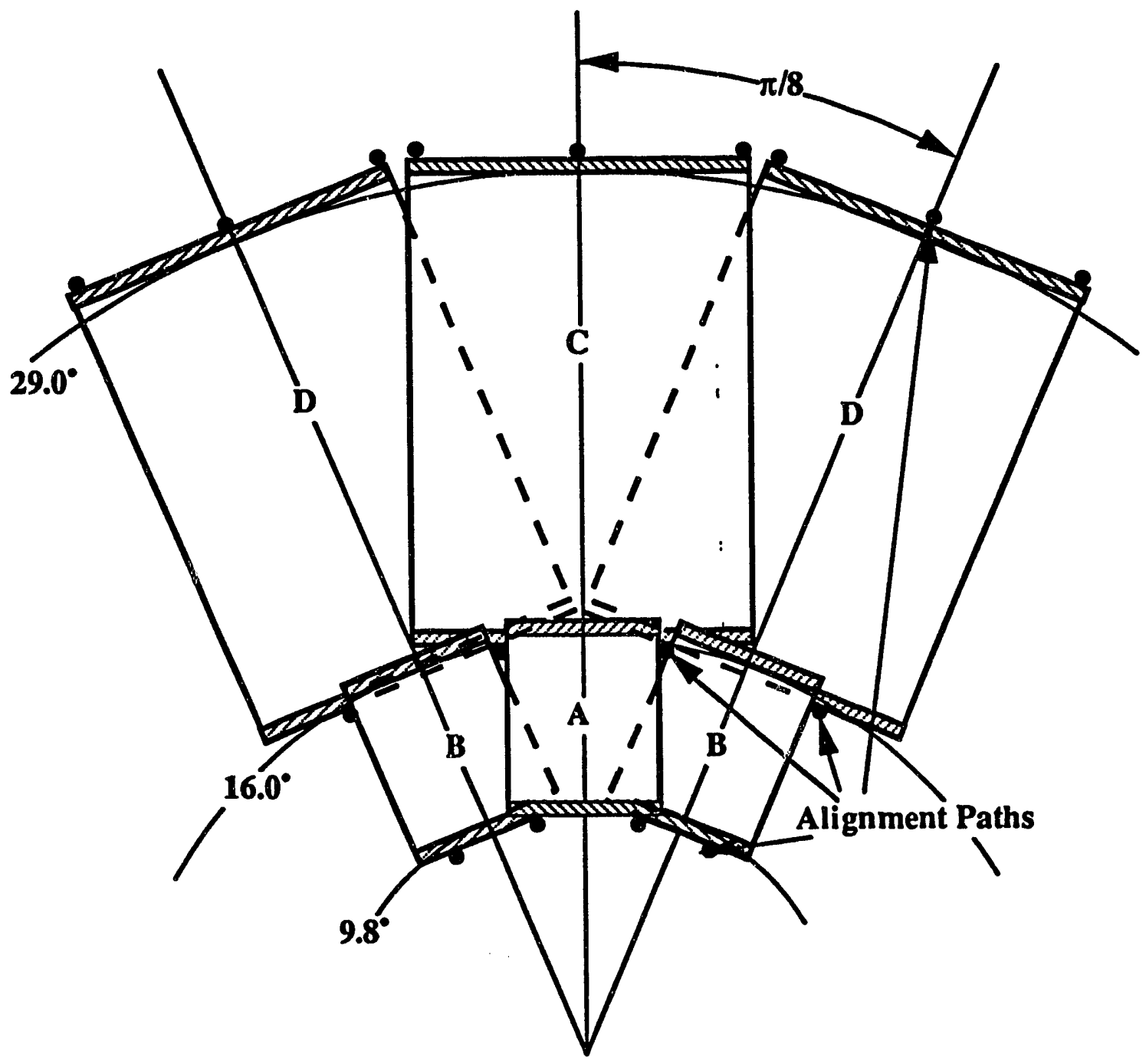

Fig. 2.6 


\section{GEM END CAP RDT CHAMBERS 16 SECTORS; 8-10-8; 25 mm Dia.}

INPUT PARAMETERS:

Tube Material, density:

Tube Wall: (mm)

M. Plate Thickness (mm), de

Case Thickness (mm), densil

\# of Sectors:

VARIABLES:

Avg. Z (m):

Inner Theta

Outer Theta

Chambers/segmnt:

\# of Layers:

\subsection{5}

\section{8}

16.0

1

8

25.4
Alum.

0.30

19.05

6.40

16
2.7

2.7

0.25

6.85

Tube Dia: (mm)

\section{RESULTS:}

Tube Length: (m)

Tubes/Chamber:

Chamb. width: (m)

\section{0}

29.0

1

8

25.4

0.7

224

0.71

0.28

Chambers/Half:

Chambers Total

Tubes/Half:

Tubes Total

16

32

3584

7168

\section{8}

464

1.51

0.28

16

32

7424

14848

11.20
16.0
29.0
10
25.4

25.4
10.60

9.8
16.0

1

10

25.4
Weight/chamber (lb)

Tubes:
Manifold plates:
Plugs:
Case:
Electronics,cable:
Total/Chamber:
Weight/Half:
Weight Total

$\begin{array}{rrrrrr}23 & 121 & 409 & 80 & 636 & 128 \\ 45 & 95 & 179 & 88 & 215 & 107 \\ 25 & 52 & 107 & 52 & 120 & 59 \\ 6 & 26 & 64 & 16 & 116 & 27 \\ 112 & 232 & 480 & 232 & 536 & 264 \\ 211 & 525 & 1240 & 467 & 1624 & 586 \\ 3372 & 8407 & 19844 & 7475 & 25976 & 9374 \\ 6744 & 16814 & 39689 & 14951 & 51953 & 18748\end{array}$

$\frac{15.60}{16.0}$

29.0

1

8

25.4 26

8

25.4

9.8

16.0

1

8

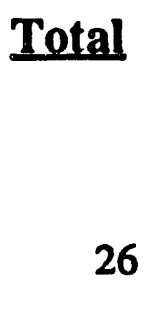

$314 \mathrm{~km}$ $\begin{array}{lll}464 & 1072 & 528\end{array}$

1.71

0.28

$0.32 \quad 0.28$

16

32

17152

34304

96
192
59392
118784

16

32

8448

16896 


\section{RDT MUONACCEPTANCE LOSSES}

\begin{tabular}{|c|c|c|c|c|c|c|c|}
\hline SOURCE & $\begin{array}{c}\text { Crack Size } \\
\text { (mm) }\end{array}$ & $\begin{array}{l}\mathbf{R}, \mathrm{Z} \\
\text { (mm) }\end{array}$ & $\begin{array}{c}\Theta \\
\text { (degree) }\end{array}$ & $\begin{array}{c}\Delta(\Theta) \\
\text { (radian) }\end{array}$ & n & $\Phi / 2 \pi$ & $\begin{array}{l}\text { LOSS } \\
(\% 4 \pi)\end{array}$ \\
\hline $\begin{array}{l}\text { Central Membrane } \\
\text { Barrel-Endcap } \\
\text { Forward Region }\end{array}$ & 250 & 4000 & $\begin{array}{c}86.4 \\
30.0 \\
8.7\end{array}$ & $\begin{array}{l}.0623 \\
.0262 \\
.1518\end{array}$ & $\begin{array}{r}.063 \\
1.317 \\
2.576 \\
\text { Bas }\end{array}$ & $\begin{array}{l}1.00 \\
1.00 \\
1.00 \\
\text { sss }\end{array}$ & $\begin{array}{l}6.2 \% \\
1.3 \% \\
2.3 \% \\
9.8 \%\end{array}$ \\
\hline $\begin{array}{l}\text { Chamber End } \\
\text { Chamber Ends } \\
\text { Alignment Path } \\
\text { Chamber Ends } \\
\text { Alignment Path } \\
\text { Alignment Path } \\
\text { Chamber End }\end{array}$ & $\begin{array}{l}100 \\
150 \\
360 \\
150 \\
470 \\
125 \\
100\end{array}$ & $\begin{array}{l}4000 \\
6000 \\
6400 \\
.000 \\
6400 \\
6000 \\
6000\end{array}$ & $\begin{array}{l}84.5 \\
57.6 \\
57.6 \\
40.4 \\
40.4 \\
16.0 \\
9.8\end{array}$ & $\begin{array}{l}.0248 \\
.0178 \\
.0401 \\
.0158 \\
.0308 \\
.0193 \\
.0162\end{array}$ & $\begin{array}{c}.096 \\
.598 \\
.598 \\
1.000 \\
1.000 \\
1.962 \\
2.457 \\
\text { Additi }\end{array}$ & $\begin{array}{l}1.00 \\
1.00 \\
0.13 \\
1.00 \\
0.13 \\
0.04 \\
1.00 \\
\text { Loss }\end{array}$ & $\begin{array}{l}2.5 \% \\
1.5 \% \\
0.4 \% \\
1.0 \% \\
0.3 \% \\
0.0 \% \\
0.3 \% \\
6.0 \%\end{array}$ \\
\hline
\end{tabular}

Table 2.3 
-

$\odot$

-

0

○

0

c

C

$i_{j}$ 


\section{(3.0) Summary of System Parameters}

The GEM Muon System is based on a large and open solenoidal magnet with an air flux return. Three superlayers of muon tracking chambers will be deployed between the calorimeter and magnet cryostat to reconstruct the sagitta in the magnetic field. Iron flux concentrators will be placed along the central axis of the magnet to create a radial component of the B-field, thereby increasing the bend power at small angles. Muon trajectories will be reconstructed using Round Drift Tubes (RDTs) from $|\eta|=$ 0 to 2.5, over most of the azimuth. The design goals for the momentum resolution of the system are for $\mathrm{Pt}=500 \mathrm{GeV} / \mathrm{c}, \delta \mathrm{Pt} / \mathrm{Pt}=5 \%$ at $|\eta|=0$ and $=13 \%$ for $|\eta|=2.5$.

The magnet will furnish a field at the IP of $0.8 \mathrm{~T}$. It will consist of a superconducting coil of $9.5 \mathrm{~m}$. radius constructed in two segments, each with a coil length of approximately $14.25 \mathrm{~m}$. The coil segments will be separated by $1.5 \mathrm{~m}$. The inner cryostat radius is $9.0 \mathrm{~m}$. and constrains the outer radius of the barrel muon chambers. The steel of the flux concentrator will be located in a cone of half-angle 9.40, (nonprojective angle) extending from $10 \mathrm{~m}$. to $18 \mathrm{~m}$. from the IP.

The resolution of the reconstructed muon momentum will be limited by the chamber resolution, the alignment precision within a superlayer and between superlayers, the bending power of the magnet (BL2), and "unreconstructable" fluctuations of the energy loss in the calorimeter. For the barrel region the lever arm, $L$, at $90^{\circ}$ will be 4.4 $\mathrm{m}$., and in the endcaps equal to, or larger than, $8.6 \mathrm{~m}$. (plus angle factors). Multiple layers of chambers will be required with exacting spatial resolutions (of order 100 $\mu \mathrm{m} /$ detector element) and systematic alignment (of order $25 \mu \mathrm{m}$ ) between superlayers to reach the desired momentum resolution. 


\section{(3.1) Physics Goals:}

(1) Provide muon identification - track penetrating 12 to $14 \lambda$ of calorimeter

(2) Charge assignment of muons

(3) Pt trigger

(4) Beam crossing time marker

(5) Muon momentum determination from a few $\mathrm{GeV} / \mathrm{c}$ to a few $\mathrm{TeV} / \mathrm{c}$. Design goal: $\delta P_{t} / P_{t}=5 \%(13 \%)$ at $|\eta|=0(2.5)$, for $P_{t}=500 \mathrm{GeV} / \mathrm{c}$.

\section{(3.2) System Parameters:}

\section{(3.2.1) Barrel Region:}

\section{Geometry:}

Rmagnet (inner radius of cryostat)

B-field at the IP

Rcalorimeter (outer radius of calorimeter)

Calorimeter thickness at $|\eta|=0$

Assumed calorimeter material

Number of sectors in $\phi$

Location of neutron shield ( $20 \mathrm{~cm}$ B-Poly)

Chamber stay-clear (neutron shield or magnet cryostat) $10 \mathrm{~cm}$

\section{RDT Chamber Parameters:}

(a) Tracking - bend plane:

Spatial resolutions (baseline assumption):

Chamber single-layer resolution

Internal chamber alignment

Superlayer-to-superlayer alignment

Tube wall thickness

Radiation length/tracking chamber layer

Tube diameter

Chamber layers/SuperLayer (SL1:SL2:S13)

Stereo configuration:

$$
\begin{aligned}
& \text { SL1 } \\
& \text { SL2 } \\
& \text { SL3 }
\end{aligned}
$$

Angle of stereo $(\alpha)$

Radii of barrel superlayer midplanes:

Barrel lever arm
$9.0 \mathrm{~m}$

$0.8 \mathrm{~T}$

$3.70 \mathrm{~m}$

$11 \lambda$

$\mathrm{Pb} / \mathrm{Cu} / \mathrm{Sc} / \mathrm{Kr}$

16

$3.70 \mathrm{~m}<\mathrm{R}<3.90 \mathrm{~m}$
$<100 \mu \mathrm{m}$ (RMS)

$<50 \mu \mathrm{m}$

$<25 \mu \mathrm{m}$

$300 \mu \mathrm{m}$

$1.14 \%$ (Al-tubes)

$2.54 \mathrm{~cm}$

$8: 10: 8$

4 axial-2 U-2V

6 axial-2 U-2V

4 axial-2 U-2V

$4 \mathrm{mr}$

SL $1=4.2 \mathrm{~m}$

SL $2=6.4 \mathrm{~m}$

$\mathrm{SL} 3=8.6 \mathrm{~m}$

4.4 to $4.5 \mathrm{~m}$ 
Half-lengths of superlayers along (Z) (approx.)

SL $1=7.0 \mathrm{~m}$

SL $2=10.8 \mathrm{~m}$

SL $3=14.4 \mathrm{~m}$

$\phi$ Widths of sectors (approx.)

SL $1=1.67 \mathrm{~m}$

SL $2=2.55 \mathrm{~m}$

SL $3=3.42 \mathrm{~m}$

Coverage:

Polar angle: (gaps not included)

$29.6^{\circ}<\theta<85.8^{\circ}$

Pseudo-rapidity:

Azimuthal angle: (goal)

$0.074<|\eta|<1.33$

$94 \%$

Number of alignment paths

768

Number of drift tubes in barrel:

229,376

(b) Tracking - nonbend plane: (RPC)

Number of RPC gaps/superlayer

Information/RPC gap

3

$X$ and $Y$

Radial locations of RPCs (chamber midplanes) (approx.)

SL $1=4.35 \mathrm{~m}$

SL $2=6.55 \mathrm{~m}$

SL $3=8.45 \mathrm{~m}$

RPC Radiation length/superlayer (2 gaps)

Chamber thickness for 2 gap package

$1.66 \%$

$3.3 \mathrm{~cm}$

Nonbend plane RPC segment widths:

Nonbend plane electronic channel count:

$\mathrm{SL} 1=3.9 \mathrm{~cm}$

$\mathrm{SL2}=6.5 \mathrm{~cm}$

$\mathrm{SL} 3=8.9 \mathrm{~cm}$

(c) Tracking-nonbend plane:(RDT)

Number of RDT stereo layers/superlayer

15,936

SL1: 4 axial - 2U-2V

SL2: 6 axial $-2 \mathrm{U}-2 \mathrm{~V}$

SL3: 4 axial - 2U-2V

Angle of stereo

Information per superlayer from stereo

$4 \mathrm{mr}$

Resolution $/ Y$ measurement

$2 Y$

$1.8 \mathrm{~cm}$ RMS

(d) Triggering: Resistive Plate Chambers (RPC)

Measurements - Bend plane:

Segment width

$1.3 \mathrm{~cm}$

Number of electronic trigger channels:

25,056

Time jitter of chamber

$<1.5 \mathrm{~ns}$

Time jitter of TO determination

$<8.3 \mathrm{~ns}$ 
(e) Triggering: Round Drift Tubes (RDT)

Bend plane segment size

Non-bend plane segment (by timing)

$1.25 \mathrm{~cm}$

$10 \mathrm{~cm}$ RMS

\section{(3.2.2) Endcap region:}

\section{Geometry:}

B-field at IP

$0.8 \mathrm{~T}$

Length of magnet cryostat

$15 \mathrm{~m}$

Z-separation of coils

$1.5 \mathrm{~m}$

Flux concentrator (FC):

Zfront

$10.0 \mathrm{~m}$

Zrear

OD-half angle of steel

ID

$18.0 \mathrm{~m}$

$9.4^{\circ \cdot}$ non-projective

$1.2 \mathrm{~m}$

Zcalorimeter (half-length of calorimeter along Z)

$5.70 \mathrm{~m}$

Calorimeter thickness at $|\eta|=\mathbf{2 . 5}$

$<17 \chi$

$16.5 \mathrm{~m}$

Number of sectors in $\phi$

16

Location of neutron shield

$5.70 \mathrm{~m}<Z<5.90 \mathrm{~m}$

Chamber stayclear

$10 \mathrm{~cm}$

Chamber Parameters (RDT):

Spatial resolutions (baseline assumption):

Chamber single-layer resolution ,

Internal chamber alignment

Superlayer-to-superlayer alignment

Tube wall thickness

Radiation length/tracking chamber layer

Tube diameter

Chamber layers/SuperLayer (SL1:SL2:S13)

$<100 \mu \mathrm{m}$ (RMS)

$<50 \mathrm{~mm}$

$<25 \mu \mathrm{m}$

$300 \mu \mathrm{m}$

$1.14 \%$ (Al-tubes)

$2.54 \mathrm{~cm}$

8:10:8

Stereo configuration:

SL1

SL2

SL3

Angle of stereo

Polar angle regions:

Channel count:

Number of RDT channels

Nonbend plane: Small angle stereo

Coverage:

Polar angle:

Pseudo-rapidity: $9.75^{\circ}<\theta<29^{\circ}$

$1.35<|\eta|<2.46$

4 axial-2 U-2V

6 axial-2 U-2V

4 axial-2 U-2V

$4 \mathrm{mir}$

$9.75^{\circ}<\theta<16^{\circ}$

$16^{\circ}<\theta<29^{\circ}$

118,784 
Azimuthal angle:

(a) Forward angle region: $9.75^{\circ}<\theta<16^{\circ}(1.96<|\eta|<2.46)$

Chamber configuration: segmented in $\phi$ :

\section{Segment A:}

$\mathrm{Z}$ location of chambers:

SL $1=6.05 \mathrm{~m}$

SL $2=10.6 \mathrm{~m}$

SL $3=15.4 \mathrm{~m}$

Segment B:

$\mathrm{Z}$ location of chambers:

SL $1=6.45 \mathrm{~m}$

SL $2=11.0 \mathrm{~m}$

SL $3=15.8 \mathrm{~m}$

(b) Outer angle region: $16^{\circ}<\theta<30^{\circ}(1.32<|\eta|<1.96)$

\section{Segment $C_{\text {: }}$}

Average $Z$ location of chambers:

SL $1=6.65 \mathrm{~m}$

SL $2=11.2 \mathrm{~m}$

SL $3=15.85 \mathrm{~m}$

Segment D:

Average $Z$ location of chambers:

SL $1=7.05 \mathrm{~m}$

SL $2=11.6 \mathrm{~m}$

SL $3=16.25 \mathrm{~m}$

Triggering: Resistive Plate Chambers (RPC)

Bend plane:

$\begin{array}{lc}\text { Segment width } 9.75^{\circ}<\theta<16^{\circ} & (1.96<|\eta|<2.46) \\ \text { Segment width } 16^{\circ}<\theta<29^{\circ} & (1.32<|\eta|<1.96)\end{array}$

$0.44 \mathrm{~cm}$

$0.60 \mathrm{~cm}$

Number of trigger channels:

71,104

Nonbend plane:

Segment width $9.75^{\circ}<\theta<16^{\circ}(1.96<|\eta|<2.46)$

SL1

$3.8 \mathrm{~cm}$

SL2

$6.5 \mathrm{~cm}$

SL3

$9.3 \mathrm{~cm}$

Segment width $16^{\circ}<\theta<30^{\circ}(1.32<|\eta|<1.96)$

SL1

$3.8 \mathrm{~cm}$

SL. 2

$6.5 \mathrm{~cm}$

SL3

$9.3 \mathrm{~cm}$

Number of nonbend plane trigger channels:

6,336

Time jitter of chamber

$<1.5 \mathrm{~ns}$ 
Note that a backup trigger based on RDTs is envisioned.

\section{(3.2.3) Grand Totals:}

Number of RDT tubes(Channels) 


\section{(4.0) Design of Round Drift Tube Chambers}

\section{RDT Design Goals and Characteristics}

The RDT design goais are to provide:

- Precise placement of wires.

- Reliable, gas tight seals for possible pressurization.

- Epoxy-bonded tubes creating a strong mechanical unit.

- Simple assembly procedures requiring a minimum of technical skill.

- A high-level of safety features for gas-flexiblity.

The Drift-tube technology and the RDT concept in particular emphasize the following characteristics:

- A high-level of redundancy is possible with a reasonable number of electronics channels at a practical cost.

- The wires are physically and electronically isolated from each other, wire breakage will cause the loss of only that tube.

- The symmetry of the round tubes will result in a drift time which can be simply and accurately corrected for the Lorentz angle effects.

- The laminated tube structure leads to a very strong and yet light package allowing large chamber structures and a minimum of alignment components.

- Projective alignment paths can be incorporated in a natural way with a minimum of acceptance loss.

- Resolution below $100 \mu \mathrm{m}$ in both the LS and Proportional modes and a demonstrated resolution improvement path through pressurization.

Two prototypes were delivered to the Texas Test Rig at the SSCL, one constructed by the Dubna group and another by the MSU group. The two detectors have been tested jointly and have achieved similar results. The mechanical construction of the MSU prototype is recognized as having several very desirable features and has been adopted by both groups. The components for a second chamber of this design have been sent to Dubna. The electronics for the MSU prototype are standard, off-the-shelf, amplifier/discriminator cards. The Dubna prototype emphasized low-noise amplifiers and has been shown to have superior performance. Elements of the Dubna design will be used to optimize the electronics for the GEM detector. 


\section{Drift Tube Design and Construction Details}

\section{A Drift-Tube Design Considerations}

The end plugs and gas manifolds are the most critical components in the design of the MSU RDT. The plugs must seal the end of the tube at up to 4 atmospheres of pressure while providing a passage for the flow of gas through the tube; they must insulate the high voltage on the anode wire from the tube and provide for electrical contact between the conductive surface of the tube and the grounded gas manifold; they must center the tube on the wire with moderate precision (to within $250 \mu \mathrm{m}$ ) and allow for the highly accurate relative positicning of the wires (to within $25 \mu \mathrm{m}$ ). The design uses standard parts, such as O-rings, wherever possible and takes advantage of modern mass production techniques, such as injection molding or automatic screw machines and our own magnetic crimping device. One important feature of the precision manifold plate design is that there are no other contributions to the systematic error in the wire placement.

\section{B. Precision Gas Manifolds}

Drawings of the gas manifold are shown in Figs. 4.0.1-4.0.2. One should note that the specification in Fig. 4.0.2 for the hole placement is a "true position" specification. The tolerance of $0.005 \mathrm{~cm}$ means that the true position of the hole centers in the manifold must lie within a circle $0.005 \mathrm{~cm}$ in diameter. We found that a local machine shop was able to hold only $.01 \mathrm{~cm}$ tolerances in producing our prototype pláies which results in a $20 \mu \mathrm{m}$ RMS on the systematic hole placement. While these plates are already within the GEM systematic error limits, we believe that alternative procedures exist for production of the manifold plates that will be more accurate and less expensive than these plates.

\section{Tube End Plug}

A section drawing of the region of the chamber near the tube ends is shown in Fig. 4.0.3. The aluminum tube is terminated by a two part plug whose details are shown in Figs. 4.0.4-4.0.6 and machined from glass filled (15\%) Delrin. We have deterinined that production of an injection-molded part is feasible and, based upon a firm quotation, will be much less costly but may require the use of a different material. The large plug part (Fig. 4.0.4) has a O-ring which insures a good gas seal on the inner surface of the aluminum tube. This plug part is crimped into the tube end by a magnetic crimping machine which was constructed at MSU specifically for this purpose. The compressive force on the tube walls needed for the crimp is produced by the interaction of a rapidly changing magnetic field (created by discharging a capacitor through a copper coil wrapped around the tube) with the induced current. The tube can be crimped in a highly repeatable fashion rith this device. The plug has been shaped so that the tube, once crimped, holds the plug firmly and the tube is wrapped neatly over the plug. This allows easy electrical contact from the tube wall to the manifold plate through a phosphor-bronze spring. 
The second part of the plug, Figs. 4.0.5 and 4.0.6, looks like a hollow bolt. Three small O-rings make up the gas seal. The threads on the bolt need not be precise as the first O-ring (the one closest to the threads) seals the bolt into the plug. The remaining two O-rings on the bolt seal above and below the gas channels in the manifold plate. Gas flows through a hole in the bolt into the tube and is removed by an identical plug at the opposite end of the tube. The assembly procedure for the chumber is greatly simplified by this two part plug: the manifold plates can be rigidly mounted on the assembly table at the correct separation, the pre-plugged tubes placed between the manifold plates and the bolt then inserted through the manifold plate into the plug.

D. Wire Location:

The wire is located in the manifold plate by a precisely drilled jewel (accurate in diameter to a few microns) which itself is positioned accurately on the centerline of the bolt near its head. At this location on the shaft of the bolt is a spherical surface designed so that the bolt fits precisely in the center of a manifold plate hole but can still change its angle somewhat to adjust to small imperfections in the bolt and plug manufacture (particularly in the threads) while not disturbing the precise centering of the jewel. The parts of the wire location system are shown in Fig. 4.0.7.

To wire a tube, the $50 \mu \mathrm{m}$ tungsten wire is attached to thin stiff rod which is then passed through the hollow of the bolts in both ends. The tungsten wire is removed from the rod and threaded throuzh the jewel hole in both pins and the pins are then pressed into the bolt heads. The wire is tensioned, held firmly by a high tensile strength silver alloy solder and then epoxy is injected into a small hole which gas-seals the pin and provides a second mechanical hold on the wire. The solder we use (Sn62) has also been recommended for its lower gold amalgamation properties.

We have made some measurements on the accuracy of the jewel hole placement in the center of the manifold plate holes. This was done by a inserting a plug in a spare manifold plate and focusing a microscope on the center of the $50 \mu \mathrm{m}$ hole in the jewel. The plug was then slowly rotated in the hole. The center of the hole in the jewel moved in a circle whose diameter was recorded. Without moving the microscope a series of other plug and jewel combinations were inserted in the same hole and similar measurements made on them. The data are shown in Fig. 4.0.8, where we have chosen a rand $m$ rotation for each jewel while the error in the concentricity is taken from the measurements. The RMS displacement of the jewel hole center along the manifold plate ( $x$-direction) from the nominal hole center is $12 \mu \mathrm{m}$. This will be a random position error and will add (in quadrature) a negligible amount to the $100 \mu \mathrm{m}$ resolution expected for the drift distance measurements. 


\section{E. Costs:}

The costs are summarized in Tables 4.0.2, 4.0.3, 4.0.4. The major labor cost is in inserting the wire in the tubes, and the major material costs are in the tubes. 


\section{Table 4.0.1}

\section{GEM RDT Design Parameters:}

No. of Chambers

Barrel 448

End Caps $\quad 192$

Total

640

No. of tubes

Barrel

229,000

End Caps

119,000

Total

348,000

Tube Material

Aluminum

Tube Diameter

$25.4 \mathrm{~mm}$

Tube Wall Thickness

$300 \mu \mathrm{m}$

Wire Diameter

$100 \mu \mathrm{m}$

Wire Tension

Operating Pressure

$1.2 \mathrm{kgm}(\mathrm{g})$

1-4 ATM

Gas Volume

$$
\text { Barrel }
$$

$460 \mathrm{kl}$

End Caps

$160 \mathrm{kl}$

Total

$620 \mathrm{kl}$

Detector Area

Barrel

End Caps

Total

$23,000 \mathrm{~m}^{2}$

$8,000 \mathrm{~m}^{2}$

$31,000 \mathrm{~m}^{2}$

Tolerances

o(wire-fiducial), random

$<50 \mu \mathrm{m}$

$<25 \mu \mathrm{m}$

o(wire-fiducial), systematic

$<100 \mu \mathrm{m}$

$\sigma$ (wire-tube axis@end of tube)

$<250 \mu \mathrm{m}$

$\sigma$ (tube sagitta)

$\sigma($ tube diameter)

$<100 \mu \mathrm{m}$

o(wire tension)

$<100 \mathrm{gm}$ (g)

Change in tube length ( $4.7 \mathrm{~m}$ tube)

$$
\text { (2) } \Delta(P)=3 \text { ATM }
$$

$0.4 \mu \mathrm{m}$

(1) $\Delta(\mathrm{T})=10^{\circ} \mathrm{C}$

$1.1 \mu \mathrm{m}$

Est. Operating Voltage (LS mode)

(1) 1 ATM

Max. Drift Time

Radiation Length/Layer

Tube

Gas(AR @ 4 ATM)

$0.04 \%$

Wire

$0.04 \%$ 
Total

Radiation Length (10 Layer Chamber)

Track Rate Limits (5\% Occupancy)

Barrel Region (Avg. $0.1<\eta<1.3$ )

Endcap Region (Avg. $1.3<\eta<2.0$ )

Endcap Region (Avg. $2.0<\eta<2.5$ )

Max. Wire Sag (4.7 $\mathrm{m}$ tube)

@ V (gravity only)

(1) $10 \mathrm{kV}$

Tube Capacitance

Characteristic Impedance
$1.14 \%$

$11.5 \%$

$120 \mathrm{~Hz} / \mathrm{cm}^{2}$

$135 \mathrm{~Hz} / \mathrm{cm}^{2}$

$330 \mathrm{~Hz} / \mathrm{cm}^{2}$

$370 \mu \mathrm{m}$

$420 \mu \mathrm{m}$

$10 \mathrm{pf} / \mathrm{m}$

$330 \Omega$ 
RDT AVERAGE CHAMBER ASSEMBLY:

16 SECTOR, 8+10+8; $25 \mathrm{~mm}$ DIA

\section{Tubes}

\begin{tabular}{|c|c|c|c|c|}
\hline Operation & & Persons & $\begin{array}{l}\text { Unit Time } \\
\text { (hrs) }\end{array}$ & $\begin{array}{l}\text { Total } \\
\text { Manhrs. }\end{array}$ \\
\hline Inspect tubes & & 2 & 0.02 & 21.8 \\
\hline Cut tubes to kneth & & 2 & 0.02 & 21.8 \\
\hline Debur tubes & & 1 & 0.02 & 10.9 \\
\hline Clean tubes & & 1 & 0.02 & 10.9 \\
\hline Flair tube ends & & 2 & 0.01 & 10.9 \\
\hline Inspect plugs & & 1 & 0.01 & 10.9 \\
\hline Put O-rings onto plugs & & 1 & 0.01 & 10.9 \\
\hline Crimp plugs into tubes & & 2 & 0.02 & 435 \\
\hline Preariure test tubes & & 1 & 0.05 & 272 \\
\hline Inspect Manifold Plates & & 2 & 2 & 4.0 \\
\hline Assemble Manifold.Plates & & 2 & 5 & 20.0 \\
\hline Setup Assembly Table & & 2 & $\mathbf{8}$ & 16.0 \\
\hline Assemble tubes into Manifold & & 2 & 0.02 & 21.8 \\
\hline Insert Wres into tubes & & 2 & 0.1 & 1088 \\
\hline Seal plug ends & & 1 & 0.02 & 109 \\
\hline Install gas fittings & & 1 & 1 & 1.0 \\
\hline Pressure test assembly & & 2 & 8 & 16.0 \\
\hline HV test & 1 & 2 & 0.02 & 218 \\
\hline Test wire tension & & 2 & 0.02 & 21.8 \\
\hline Ascemble Case & : & 2 & 10 & 20.0 \\
\hline Prepare Readout Boards & & 1 & 1 & 17.0 \\
\hline Test Readout Boards & & 1 & 0.5 & 8.5 \\
\hline Assemble Powrer Buses & & 2 & 4 & 8.0 \\
\hline Assemble R.O. Boards & $!$ & 1 & 1 & 17.0 \\
\hline Assemble HV Boards & & 1 & 1 & 17.0 \\
\hline Final testing & & 2 & 8 & 16.0 \\
\hline Package for Shipping & & 2 & 8 & 16.0 \\
\hline Total & & & & $\overline{530}$ \\
\hline Time/Tube & & & & 0.76 \\
\hline Time/Chamber & & & & 117 \\
\hline
\end{tabular}


BARREL RDT COST ESTIMATE $8+10+8 ; 25$ MM TUBES

NUMBER OF CHAMBERS:

NUMBER OF CRANNELS:

ETEM

TUBES (Avg. $3.7 \mathrm{~m}$ )

PLUGS

WIRE GUIDES

WIRE (m)

MANTHOLD PLATES

CASE

R.O. BOARDS

TEST/ASSEMBLY EQUIP.

\begin{tabular}{rrrr} 
REQ'D (K) & COST(S) & NOTE & TOTAL(SK) \\
229 & 26.12 & 1 & 5992 \\
459 & 1.70 & 1 & 780 \\
459 & 1.50 & 1 & 688 \\
1147 & 0.20 & 2 & 229 \\
0.896 & 2500 & 2 & 2240 \\
0.448 & 300 & 2 & 134 \\
7.168 & 50 & 3 & 358 \\
& & 3 & 200 \\
\cline { 3 - 3 } TOTAL COMPONENTS & & & 10622
\end{tabular}

228 25 4 5702

TOTAL PARTS + LABOR

NOTES:

1. BASED ON ESTIMATE FROM SOURCE

2. BASED ON PROTOTYPE, NO ALLOWANCE FOR QUANTITY DISCOUNT OR ALTERNATE METHODS

3. ESTMATE

4. ESTIMATE (AVg. 512 tubes/chamber $₫ .76 \mathrm{hrs} /$ tube $+120 \mathrm{hrs} /$ chamber) 


\section{END CAP RDT COST ESTIMATE}

\section{8+10+8; 25 MM TUBES}

NUMBER OF CHAMBERS:

NUMBER OF CHANNELS:

THOM

TUBES (Avg. $2.7 \mathrm{~m}$ )

PLUGS

WIRE GUIDES

WIRE (m)

MANIFOLD PLATES

CASE

R.O. BOARDS

TEST/ASSEMBLY EQUIP.

\begin{tabular}{rrrr} 
BOQD (K) & COST(S) & NOTE & TOTAL(SK) \\
118.8 & 19.06 & 1 & 2264 \\
237.6 & 1.70 & 1 & 404 \\
237.6 & 1.50 & 1 & 356 \\
475.1 & 0.20 & 2 & 95 \\
0.384 & 2500 & 2 & 960 \\
0.192 & 300 & 2 & 58 \\
3.712 & 50 & 3 & 186 \\
. & & 3 & 200 \\
\hline & & 3523
\end{tabular}

113

25

4

2830

TOTAL PARTS + LABOR

NOTES:

1. BASED ON ESTIMATE FROM SOURCE

2. BASED UN PROTOTYPE, NO ALLOWANCE FOR QUANTITY DISCOUNT OR ALTERNATE METHODS

3. ESTIMATE

4. FSTIMATT (Avg. 618 tubes/chamber @. $76 \mathrm{hrs} /$ tube $+120 \mathrm{hrs} /$ chamber) 
SECTION DET AIL
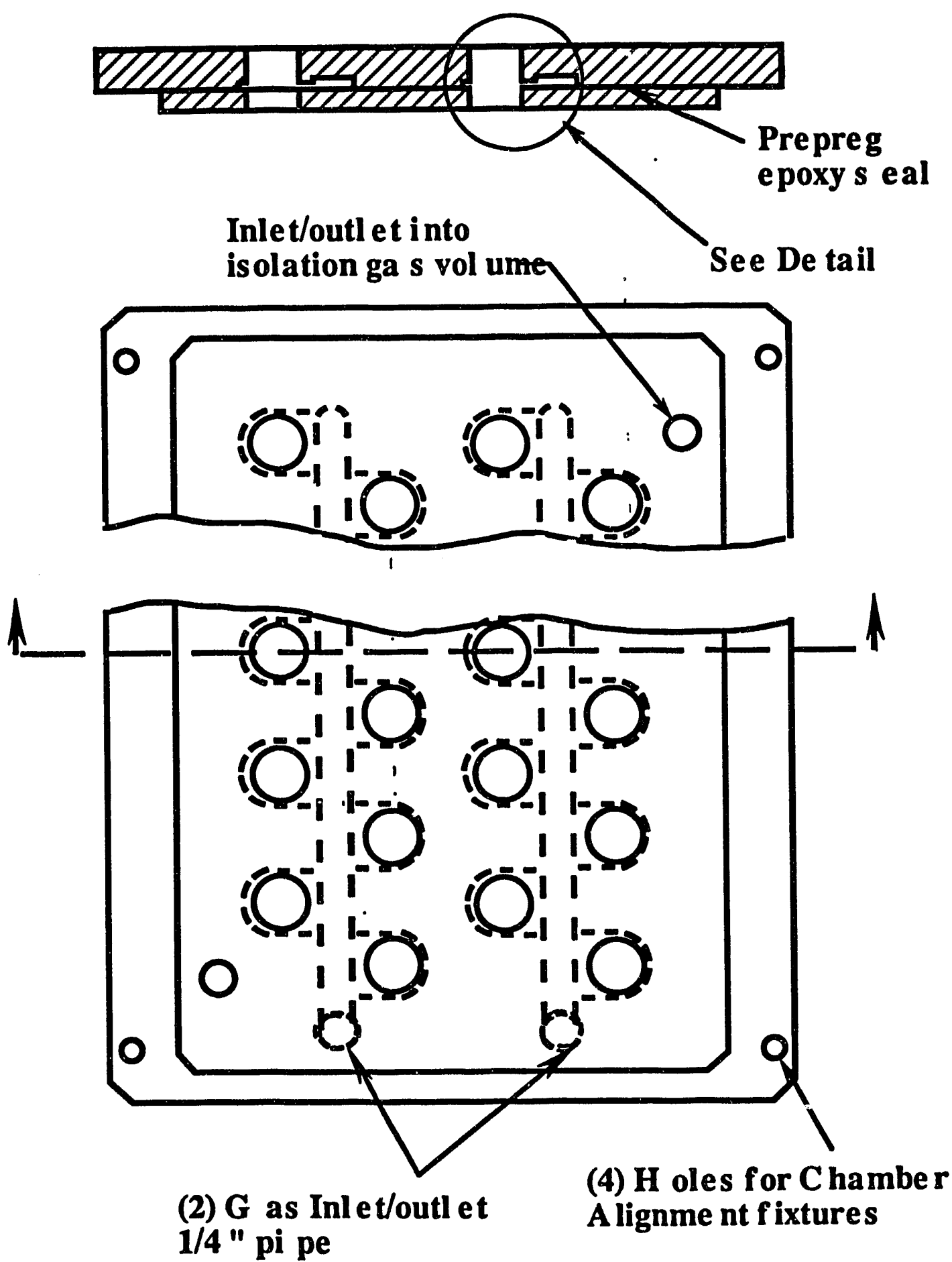

Fig. 4.0.1 


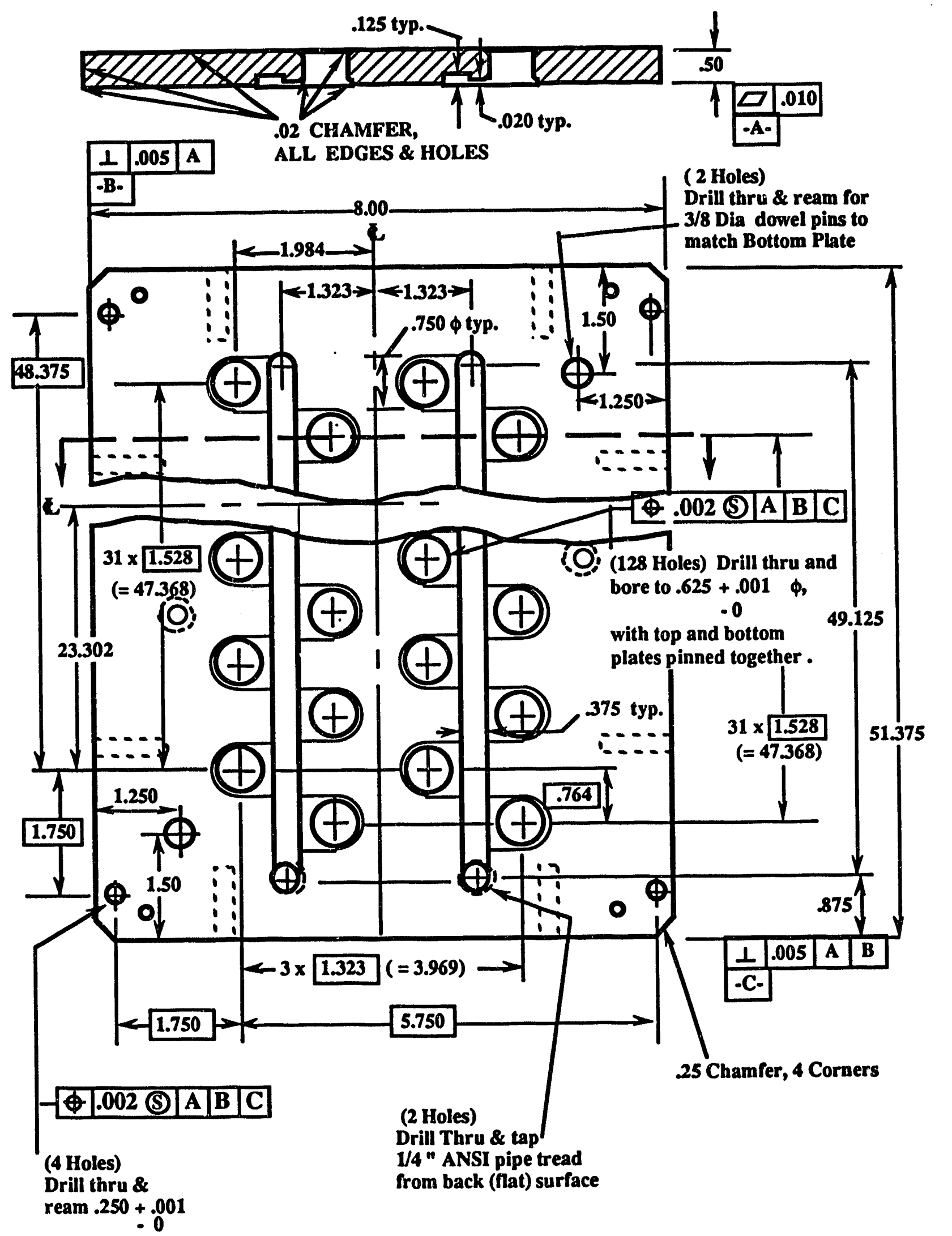

Fig. 4.0 .2 


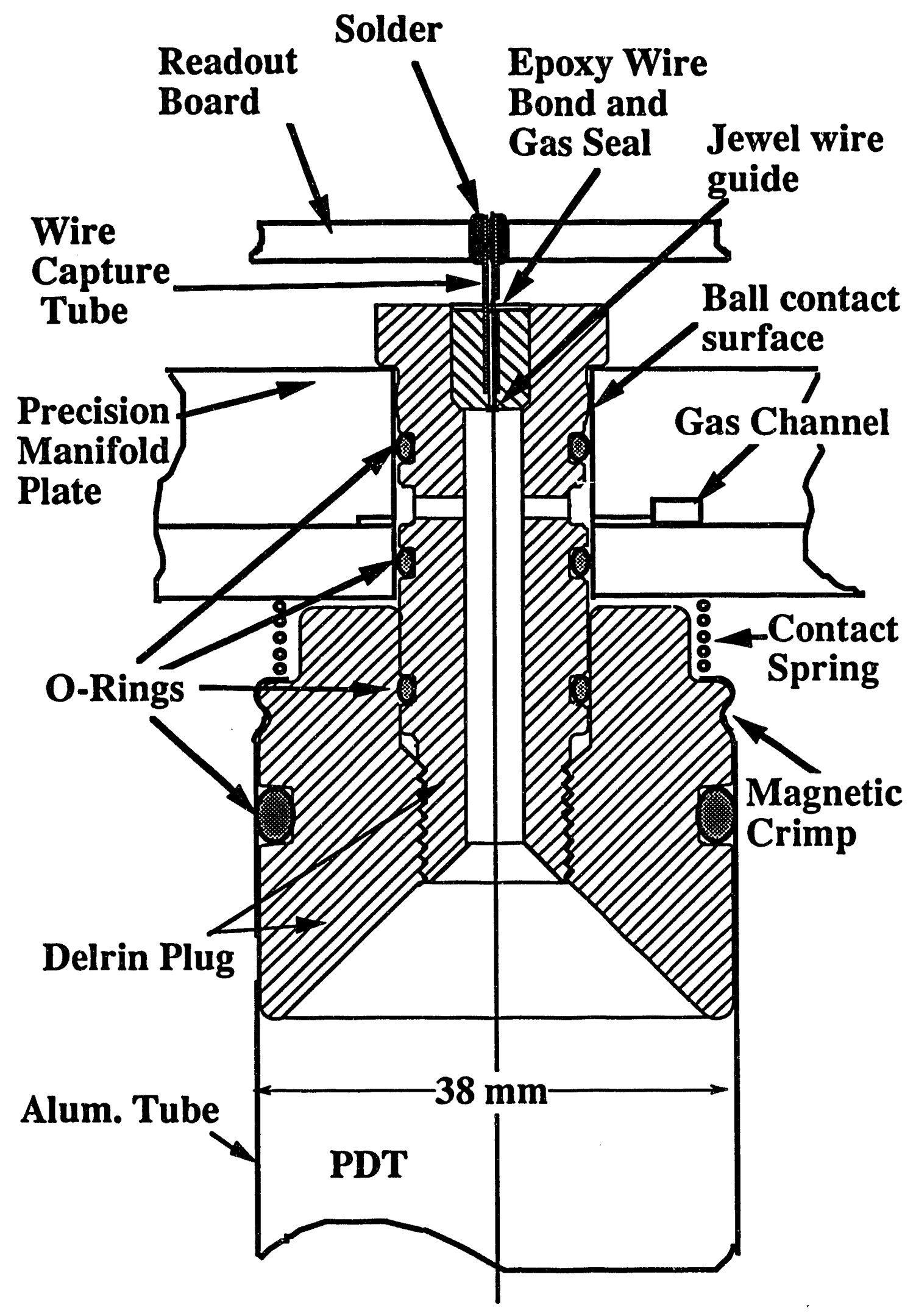

Fig. 4.0 .3 


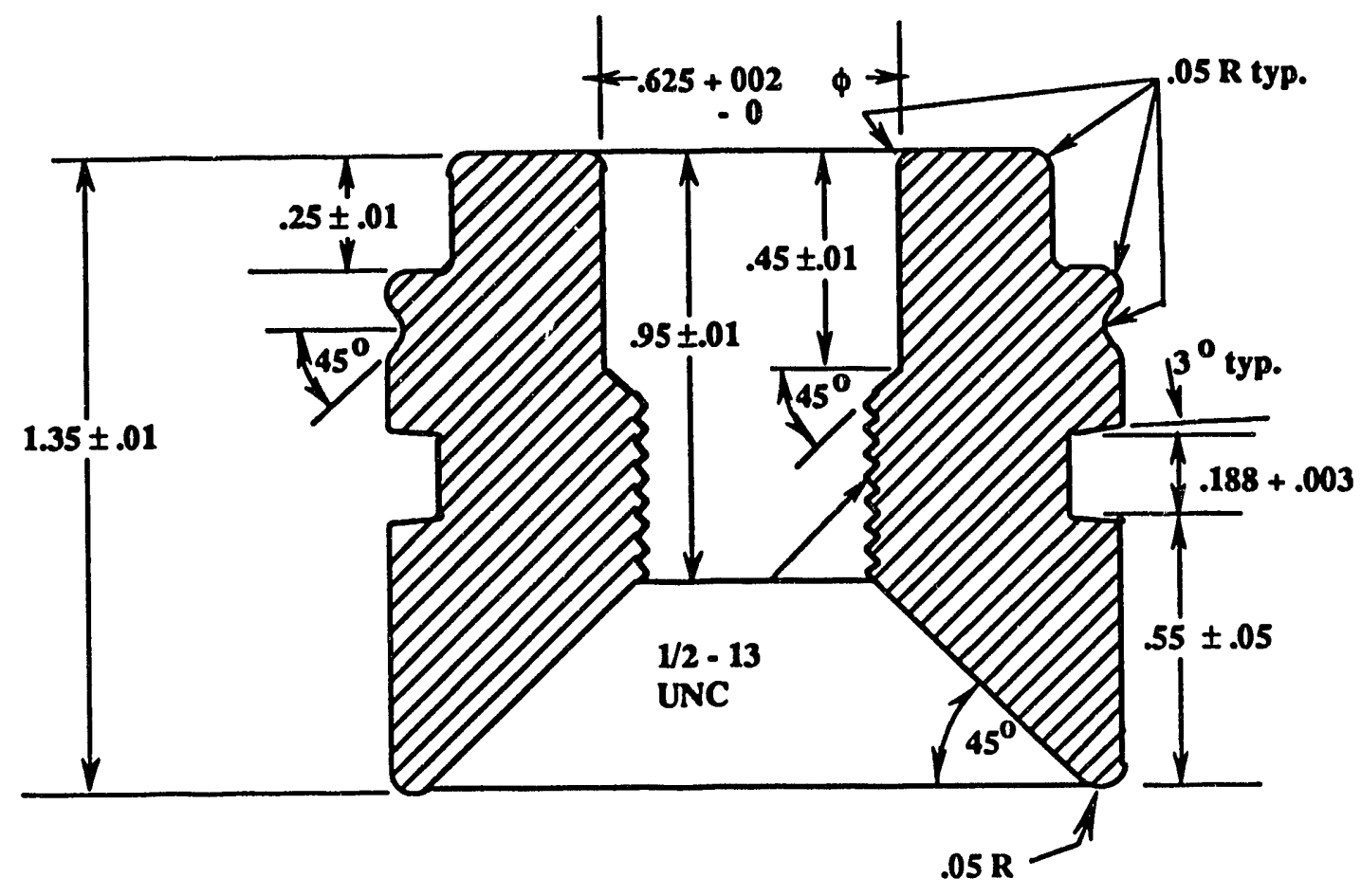

Fig. 4.0 .4 


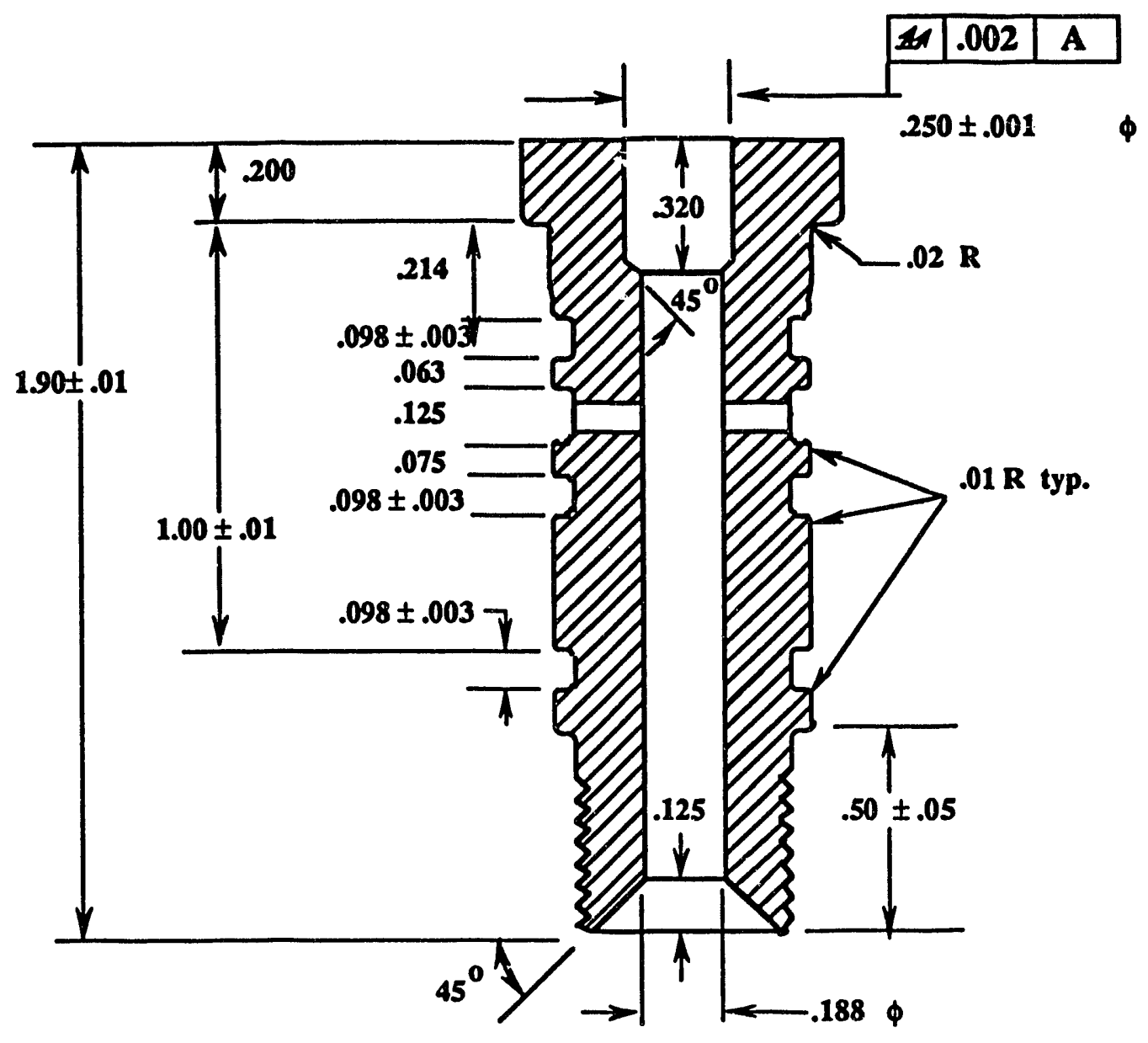

Fig. $4 \cdot 0.5$ 

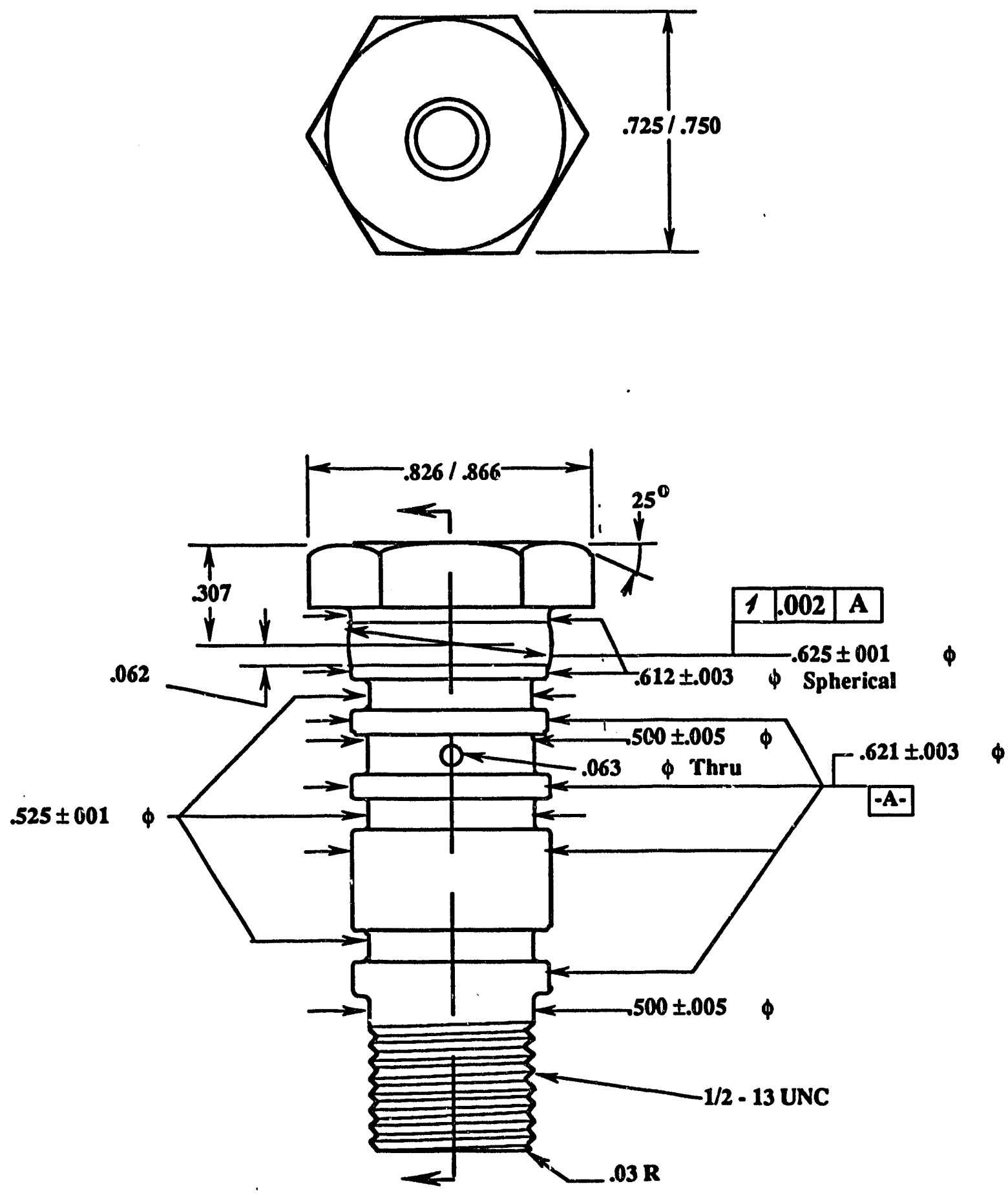

Fig. 4.0 .6 


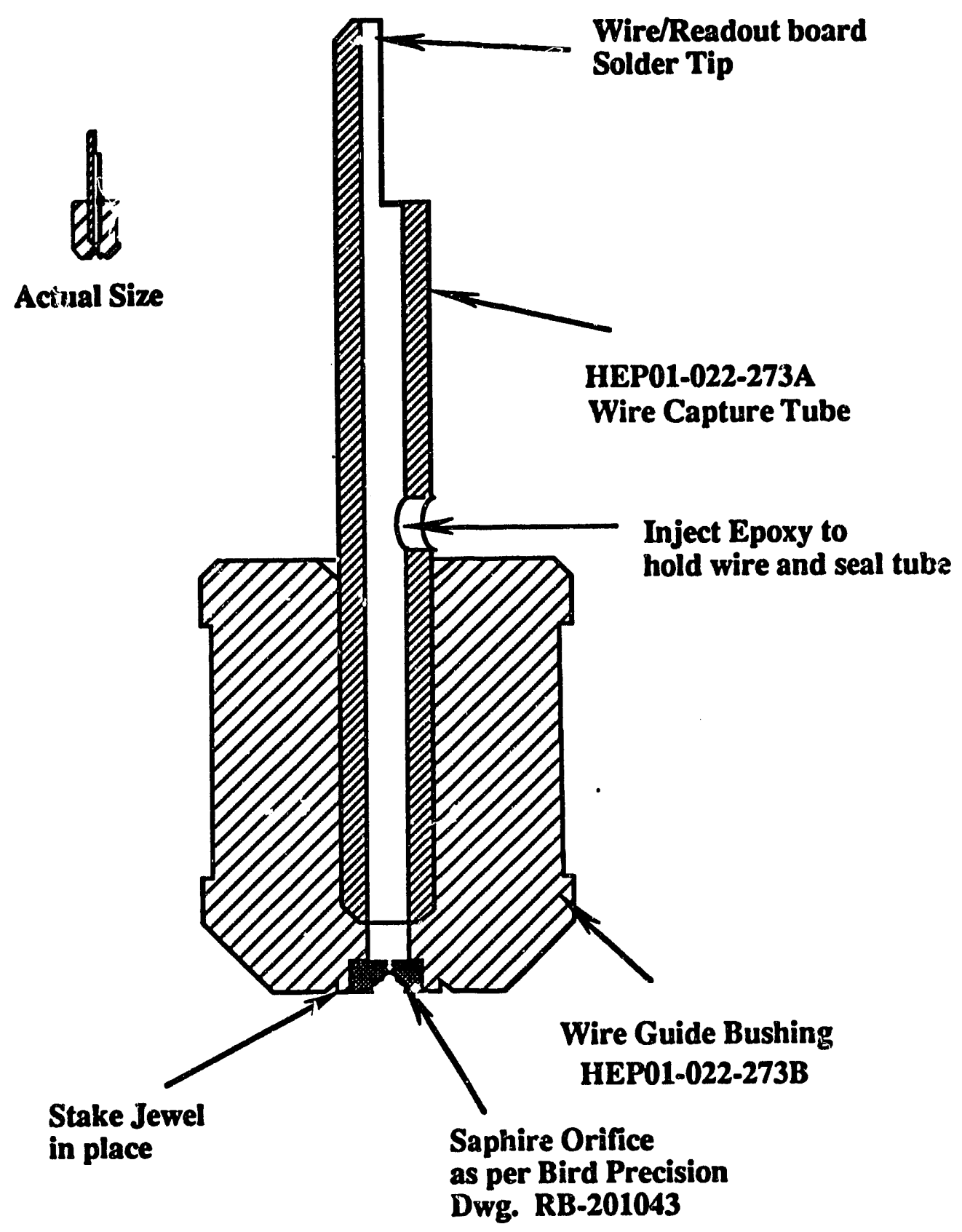

c

Fiร. 4.0 .7 

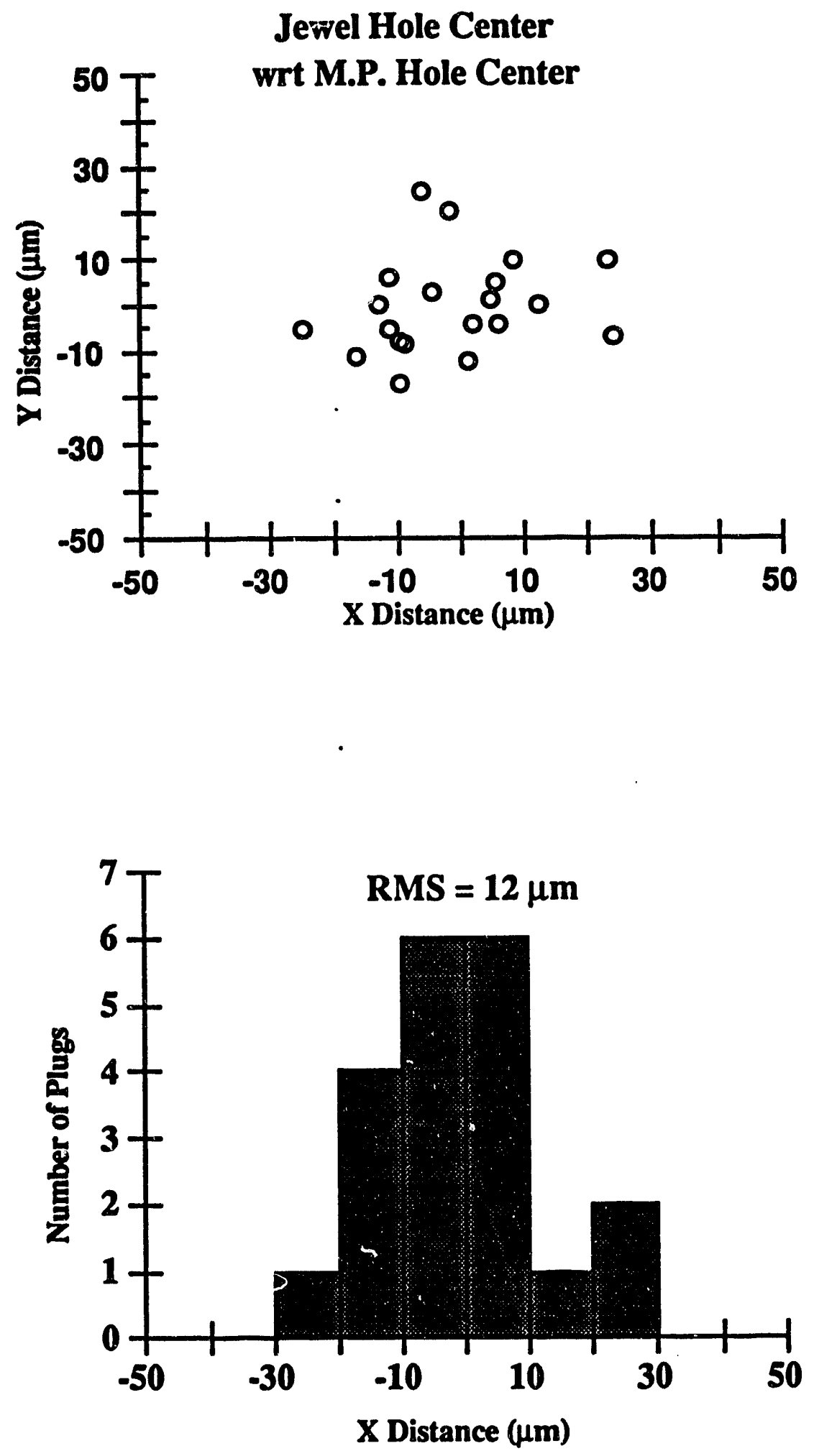

Fig. 4.0 .8 


\section{(4.1) RDT Stereo Views}

\section{Tracks from Stereoscopic Measurements:}

In a sector a local coordinate system is defined with the z-axis parallel to the beam, which is the direction of the wires in $x$-planes (axial), and with the $x$-axis in the plane of and perpendicular to the wires. Tracks are defined by the parameters: $x(r), \theta(r), x_{0}$, and $z_{0}(x$ and $z$ at $r=0)$, where the magnetic field is assumed to have only small components in the $x$, and $r$ directions. For tracks of reasonable momentum the path along the helical trajectory is short enough to consider $\theta(r)=$ constant where the constant is called the $\theta$-parameter of the track. Using the $x$-coordinate from the projection of the track determined from the $X$ planes and the stereo measurements from a $U-U^{\prime}$ or a $U-V$ pair of planes the $z$-coordinate of the track as it passed through those planes can be determined as shown in Figure 4.1.1.

a)

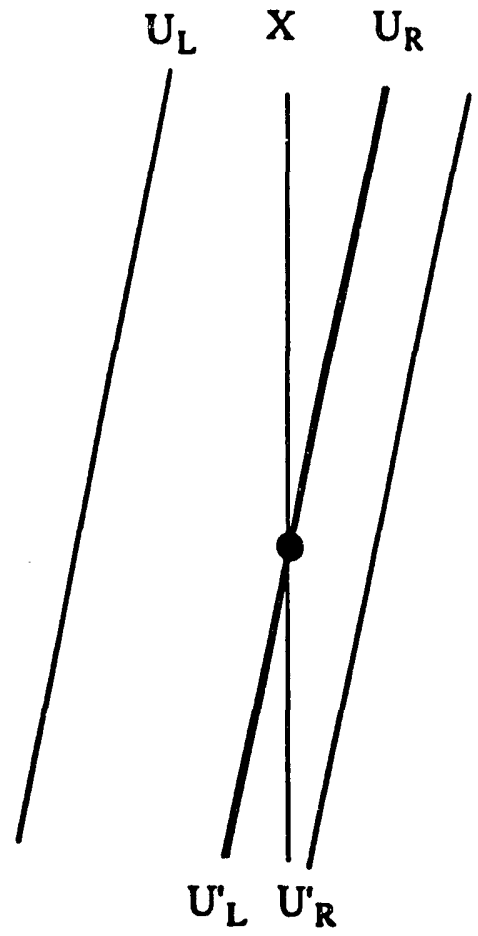

b) $\mathrm{X}-\mathrm{U}-\mathrm{V}$
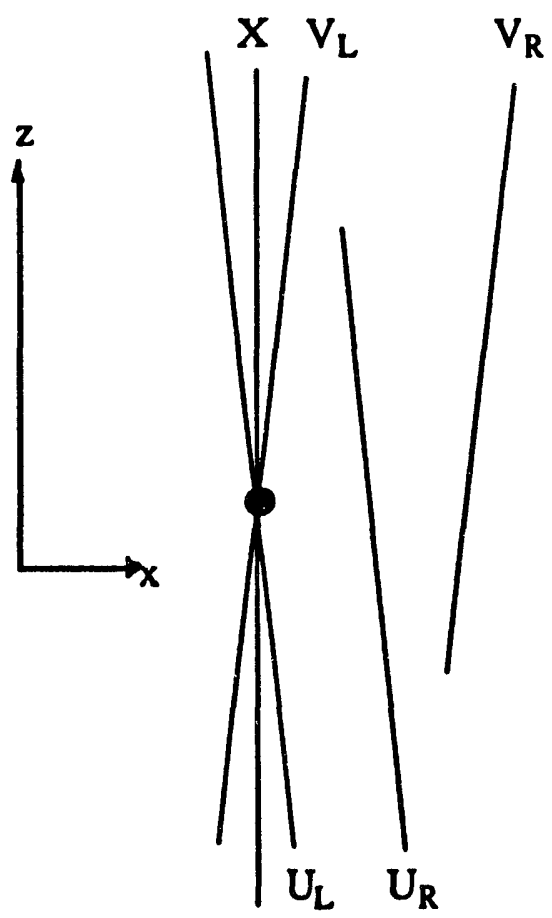

C

a) The $z$-coord. determined from the $x$-coord. and staggered $U-U$ ' drift tubes. b) The z-coord. determined from the $x$-coord. and a $U-V$ pair of drift tubes.

Fig. 4.1.1 


\section{How many planes in total?}

The RDT baseline has the planes arranged in an 8-10-8 layout in an 4-6-4 arrangement of axially oriented wires while the small angle stereo layers are 4-44 with a UU'-VV' quartet at each superlayer. The $z_{0}$ and $\theta$-parameters can be determined either by generating space points in each superlayer or by reconstructing the track in any two of the three views (the axial and the two stereo views) and combining the results. The momentum resolution is optimized by a multi-parameter fit for the momentum, $x_{0}$ and $z_{0}$, and $\theta$-parameters. The resolution obtained in this way from the 26 measurements along a track is nearly be indistinguishable from a purely axial system. If the number of planes must be reduced (cost) a system with only two views (reducing the number of stereo planes to 2 at each superlayer (but adding back 2 axial planes at superlayer 1 ) in an 8-8-6 would be only marginally worse in resolution due to the relative lack of stereo ambiguities in a small angle system, however, the redundancy would suffer considerably, making the system less robust at high luminosities.

\section{What Stereo Angle?}

A plane positioned so that its wires are at a small stereo angle $\psi=4 \mathrm{mr}$ with respect to the axial wires will yield a $z$-measurement with a resolution of $\sigma_{z}=\sigma / \psi$ $=2.5 \mathrm{~cm}$, where $\sigma$ is the drift distance resolution (the $x$-coordinate is obtained accurately from the axial wires). A $4 \mathrm{mr}$ stereo angle implies that the tubes displace by less than one tube diameter in the longest chambers. With 4 stereo planes at each station the resolution in $\mathrm{z}$ at each superlayer would be better than $1 \mathrm{~cm}$ after using the constraints of a straight line in the $\theta$-view. Larger stereo angles should not be necessary since these resolutions are already much better than the requirements of the GEM muon system. At smaller stereo angles the zcoordinate resolution would be worsened but fewer stereo measurements would be subject to $1-r$ ambiguities as shown in Figure 4.1.2. 


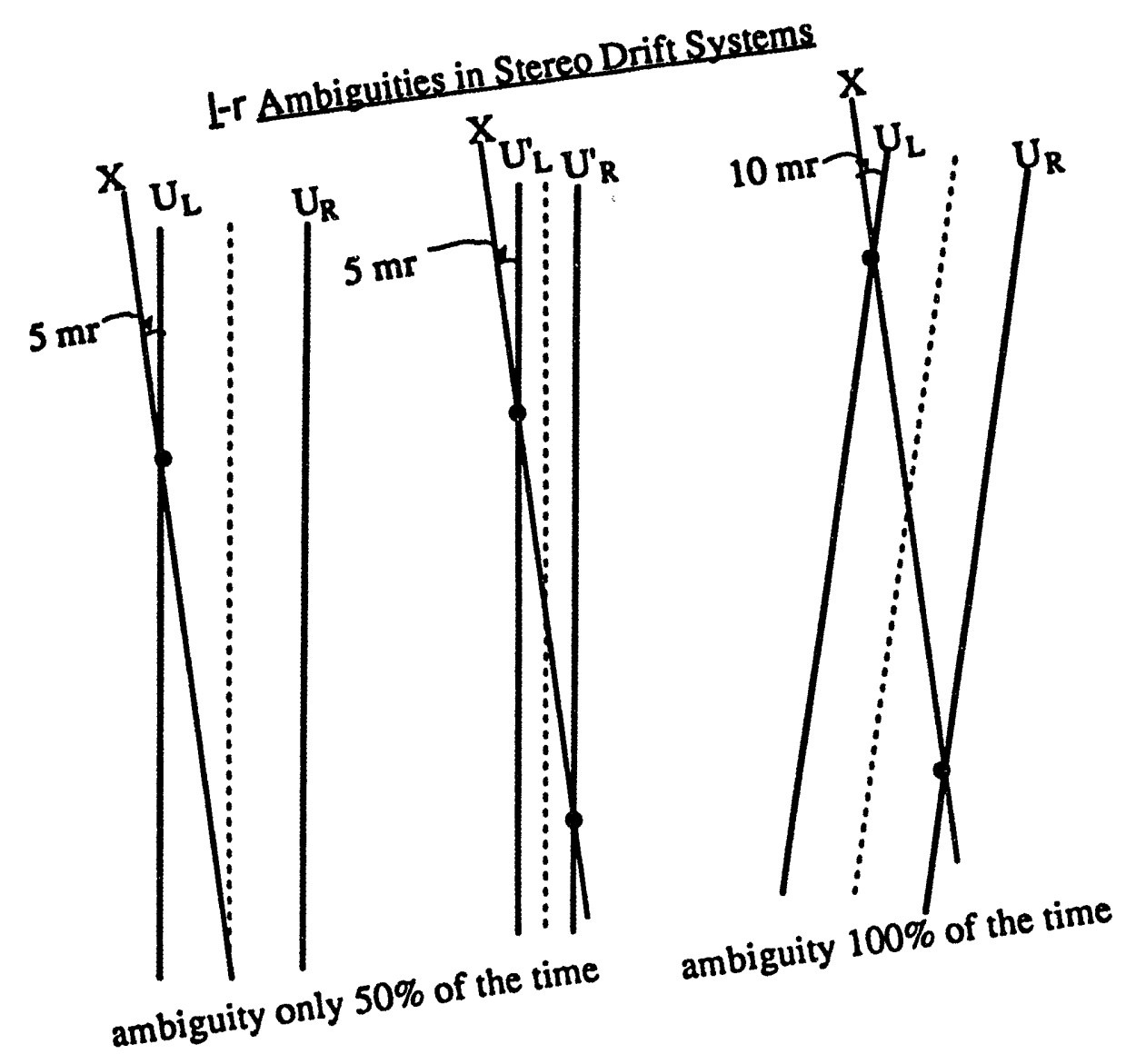

mall angle stereo (1/2 tube width over the full
$50 \%$ of the time Small angleads to l-r ambiguities only $50 \%$ of the the width over while a stereo angle twice as lambiguities $100 \%$ of the the full length) time

F1g. 4.1 .2 


\section{(5.0) RPC Technology}

Resistive Plate Chambers (RPCs) have many properties which make them an ideal technology for triggering a large muon system. The RPCs are intrinsically fast, easy to construct and operate, and inexpensive to build. The readout can be accomplished by pickup strips which inductively detect the avalanche induced by the muon upon traversal of the $2 \mathrm{~mm}$ chamber gas gap. We have demonstrated that RPCs have the required time and space resolution and can be operated simultaneously with the RDTs - validating our interest in this technology. The present difficulty is that the chambers have rate limitations which make them an uncertain choice for the high rate environment at GEM - especially for application in the endcaps. More R\&D is needed to really be certair that they will meet the baseline requirements for GEM.

The following is a brief description of the RPC technology. More details can be found in the Appendix A.

\section{A. Design}

\section{General Design Concept}

The current standard (Italian) design is two meters long by one meter wide with a sensitive gas gap of 2 millimeters. There are no wires or conductors anywhere in the sensitive region. The walls of the chamber - in contact with the gas of the sensitive volume - consists of a semi-conductor (Bakelite, for example) with a bulk resistance of about $10^{11} \Omega$ centimeters. The dimensional tolerances are modest. The thickness of the Bakelite plate is to first order not relevant for the proper operation of the RPC. The combined flatness and spacing requirements between the plates are \pm 200 microns. This requirement is trivial to maintain over arbitrarily large areas by using PVC spacers $2 \mathrm{~mm}$ thick and $11 \mathrm{~mm}$ in diameter spaced on a $10 \mathrm{~cm}$ grid. An ionizing particle passing through the sensitive volume ( $2 \mathrm{~m} \times 1 \mathrm{~m} \times 2 \mathrm{~mm}$ ) breaks down the gap and creates an electrical discharge. This discharge is very fast (a few nanoseconds). The discharge is capacitively coupled to pick-up strips which are located outside the semiconductor walls of the gas cell.

When the strip is terminated with a $50 \Omega$ load the voltage pulses across the termination are typically 0.5 volts high with a rise time of $2-3$ ns. A typical full width at half height (FWHM) is about 10 ns and the measured rise time jitter is less than 1.4 ns. Figure 5.1 is a schematic of the "standard" Italian RPC.

RPCs are used to perform the fast triggering necessary to identify the SSC beam bunch crossing associated with a particular physics event, as well as the muon momentum trigger (Level 1 trigger) for identifying muons of sufficient momentum. In the barrel region of the GEM Detector, bunch crossing identification is performed using straight line fits of tracks registered on projective strips oriented in the $\theta$ 
direction (non-bend plane), with widths of $3.9,6.5$, and $8.9 \mathrm{~cm}$, for inner, middle, and outer super-layers, respectively. Muon momentum measurements are made using strips of $1.3 \mathrm{~cm}$ width oriented along the $z$ direction (bend plane). The strip widths are chosen to provide the necessary precision for track identification. In the case of the bunch-crossing identification, the wide strips are all that is necessary to identify a straight line track. In the case of the muon momen. $\mathrm{dm}$ identification, $1.3 \mathrm{~cm}$ strips are sufficient to provide sagitta measurements of the necessary precision to identify muons of particular momenta, for example, $>10 \mathrm{GeV} / \mathrm{c}$, or $>50 \mathrm{GeV} / \mathrm{c}$. This momentum identification is based on the measurement of the muon passage through a set of strips in the three super-layers and the strip displacement from a straight line as projected from the origin through the outer super-layer strip. The RPC strip widths in the end cap region are slightly different than in the barrel and the operation of the RPC trigger for both the barrel and end cap is described in greater detail in a later section.

Reading out an RPC is a simple matter of defecting the induced charge on the cathode strips, identifying the time of arrival of the charge, and the spatial coordinates of the strip. The RPC operation (spark chamber) implies that it is essentially a digital device. The critical conditions for RPC operation are 1) stability of materials used in the RPC, 2) stability and uniformity of the gas supplied to the RPC , 3) uniformity of the gas gap within the RPC that gives rise to the avalanche breakdown and the subsequent induced pulse on the pick-up strips, and 4) saturated rate capability in large particle fluxes, related to the bulk resistivity of the semiconducting material used for the resistive plates. The RPC system, being a somewhat less mechanically precise system than the proposed drift tube systems, uses much simpler manufacturing methods than the precision drift tube technologies being considered for the GEM Muon System.

\section{High Rate RPC for GEM Muon Trigger System}

The conditions in the GEM Detector environment demand that RPCs be capable of operating at higher rates than currently available with the standard Bakelite design. This is because there will be a large background of neutrons and charged particles in the barrel and end-cap regions of the detector. Neutron fluxes of about $10^{4} \mathrm{~Hz} / \mathrm{cm}^{2}$ are expected, for example, in the barrel region first super-layer, with about a factor of 10 higher rate in the forward direction, at a luminosity of $10^{33}$ (not taking into account the possible addition of neutron shielding which could reduce the neutron flux by an additional factor of 20-30). Bakelite RPCs have been measured to have an efficiency for neutrons of about $0.5 \%$, which implies an RPC singles rate due to neutrons of about $50-500 \mathrm{~Hz} / \mathrm{cm}^{2}$. Bakelite RPCs also have a measured rate capability at $97 \%$ plateaued efficiency of about $50-100 \mathrm{~Hz} / \mathrm{cm}^{2}$. Therefore, it is desired that RPCs used in the GEM Detector have higher rate capability by a factor of $10-100$. Our R\&D program indicates that this rate capability is now understood to be solely a function of the bulk resistivity of the resistive plate material. 
Fig. 5.2 shows a SPICE model of a single layer RPC. The resistive plates are modeled as a resistor and capacitor in parallel, with the resistor determined by the measured bulk resistivity of the material and the thickness. The gas gap is modeled as a simple capacitor. A number of materials were studied for use in RPCs. Our model can be used to predict the dynamic behavior of the RPC when a spark discharge occurs in the gas gap. Fig. 5.3 shows that the model predicts a characteristic "recovery" time, associated with the re-establishment of the electric field in the plate after the discharge occurs, which varies widely for different materials. Note that the curves in Fig. 5.3 show the recovery time of a region around the spark discharge in the RPC and do not represent the signal pulse shape out of the RPC. We arbitrarily define the recovery time as the time between the $10 \%$ and $90 \%$ amplitude points on the curves shown in Fig. 5.3.

This recovery time is directly related to the saturated rate capability of the RPC as discussed below. The following is a simple model that lets one deduce the rate of a counter at a fixed efficiency given the saturation counting rate. This model usually underestimates the rate for high efficiencies.

Assume:

$$
\begin{aligned}
& \mathrm{Rs}=\text { saturation rate } \\
& \operatorname{Re}=\text { rate at a fixed efficiency } E \\
& \operatorname{Re}=\operatorname{Rs}(1-\mathrm{E})
\end{aligned}
$$

This equation is trivial to derive but it is only an approximation as it assumes a linear relation between saturation counting rate and the period of inefficiency of the counter. Note that this equation predicts that there is no rate for which a counter can be $100 \%$ efficient, which is true as every counter has to have a dead time and regardless of rate there is a non-zero probability to have two random counts within this dead time. If one assumes $\mathrm{E}=0.95$, which is the number that is normally used for this equation, then for $\mathrm{Rs}=20,000 \mathrm{~Hz}$ we can assume that the counter will operate at $1,000 \mathrm{~Hz}$. Because of the approximation previously mentioned, this derived rate is actually an underestimate of the real rate.

Fig. 5.4 shows the results for saturated rate measurements for different resistive materials compared to the inverse of the recovery time. The measured rates are seen to be proportional to the calculated time response of the material, indicating that we can understand the operation of the RPC based on this simple model and the knowledge of the bulk resistivity of the material. From Fig. 5.4 we see that two special plastics loaded with conducting polymer exhibit very high saturated rate capability compared to glasses and Bakelite, with rates approaching $15 \mathrm{kHz} / \mathrm{cm}^{2}$. One plastic is an ABS-based plastic and the other is a PVC-based plastic. We have measured the dielectric strength of the ABS plastic tc be superior to the PVC plastic. A number of RPCs constructed from ABS plastic have been built and tested (the largest being $1.2 \mathrm{~m} \times 2.4 \mathrm{~m}$ ). 
R\&D results (detailed in an Appendix) from measurements performed on a number of different RPCs indicate that plastic RPCs operate identically to Bakelite RPCs with regards to pulse width, pulse height, and rise-time jitter. In addition, noise measurements have been made and the best chambers exhibit a noise rate of about $0.5 \mathrm{~Hz} / \mathrm{cm}^{2}$ for a $15 \mathrm{mV}$ discriminator threshold. This noise rate is achieved for plastic sheet without any special surface preparation, as compared to Bakelite chambers, which require a special coating of linseed oil on the inner surfaces in order to operate. The noise rate of $0.5 \mathrm{~Hz} / \mathrm{cm}^{2}$ is roughly a factor of two worse than the best Bakelite/linseed oil chambers and is about 20 times the cosmic ray rate.

The ABS plastic RPC appears to exhibit some variability in operation associated with initial turn-on and subsequent voltage changes. This variability is manifested as an increase in noise rate and exhibits a characteristic settling time of the order of hundreds of minutes. Once the RPC is stabilized the noise rate is reduced to the value quoted above. Another characteristic of the ABS plastic is a variability in saturated count rate associated with turn-on and subsequent voltage changes. This variability appears to be associated with a change in the bulk resistivity of this particular plastic and is possibly due to polarization effects. For example, it is observed that the saturated rate capability of RPCs constructed from ABS plastic is reduced from about $10 \mathrm{kHz} / \mathrm{cm}^{2}$ to about $1 \mathrm{kHz} / \mathrm{cm}^{2}$ over a period of a few hours. Also, the noise rate drops to a final value of about $0.5 \mathrm{~Hz} / \mathrm{cm}^{2}$. Once the $\mathrm{RPC}$ reaches this point it is stable, as shown by measurements of an RPC under constant voltage over a 6-day period.

There are a number of other semiconducting plastics that will be studied for use for high rate RPCs and it is expected that the variability discussed above will be overcome with the choice of the proper plastic. In addition, plastics with even lower bulk resistivities (in the range of $10^{8} \mathrm{ohm}-\mathrm{cm}$ ) have been identified. Based on our understanding of RPC operation, these lower resistivity plastics will provide RPC operation with correspondingly higher saturated rate capability - by as much as a factor of ten better than ABS plastics. In this event, sufficient rate capability for GEM will be insured even with small (less than a factor of ten) changes in resistivity. We will next describe the construction of RPCs based on these new plastic materials.

\section{B. RPC Construction}

\section{Manufacturirig}

Our manufacturing philosophy is to build as large a chamber as possible for each super-layer following the same dimensions and lay-outs as for the RDT tracking chambers. The materials used in the individual chambers should be able to be assembled into a self-supporting structure that first and foremost maintains the gas gap dimensions with a tolerance of about $\pm 5 \%$, or, in the case of a $2 \mathrm{~mm}$ gas gap, \pm $100 \mu \mathrm{m}$. Similar dimensional tolerances are necessary for the distance between the pick-up strip electrodes and their ground plane, in order to control the strip 
impedance. The gas gap is maintained by gluing $2 \mathrm{~mm}$ thick, $1 \mathrm{~cm}$ diameter disk spacers every $10 \mathrm{~cm}$ or so in between the laminated sheets. The strip-electrode-toground plane gap is maintained by using a rigid foam sheet to form the proper impedance transmission line. Because of this design flexibility the RPC can actually be deformed substantially from a flat plane and still operate correctly.

Similarly, the tolerance associated with the placement of the pick-up strips is defined by the desired measurements of timing (bunch crossing) and momentum (sagitta). This tolerance as we see it implies strips to be located also to about $\pm 500 \mu \mathrm{m}$, a not too difficult tolerance to achieve using standard commercially available techniques for metallization of mylar sheets.

Materials stability is an issue for long term performance of the RPCs. Plastics degrade over time due to out-gassing of plasticizers, interaction with ambient UV light, temperature fluctuations, radiation, and, in the case of RPCs, uniformity and stability of the bulk resistivity of the resistive plates and interaction of the plates with spark discharges. Proper materials choices and design will help to insure the long term stability of the RPCs. We can benefit from the experience of other RPC systems that show long term (years) operation of RPCs without degradation, albeit under lower rate conditions than that expected at the SSC. Aging tests are easily performed in R\&D to determine the proper long term operation of RPC materials.

Fig. 5.5 shows a blow-up of a two-gap RPC design. The spacers produce about a 1\% dead area; however, the size and spacing of these spacers has not been optimized and should be studied using finite element-analysis to assess the minimal configuration required to maintain a gas gap to the required $2 \mathrm{~mm} \pm 0.1 \mathrm{~mm}$. The two-gap design we have chosen eliminates this $1 \%$ dead area by having two gas gaps with the spacers in one gap staggered with respect to the spacers in the second gap. This double gap construction also has the virtue of providing practically $100 \%$ redundancy for the RPC system. We are proposing to build a three-gap RPC stack, i.e., three independent RPCs within a single chamber. The three RPCs can be used to perform a two-out-of-three coincidence with a very tight timing window of about 20 ns by virtue of the inherent speed of the RPC. This provides a very low accidental trigger rate due to neutrons and charged particles conspiring to mimic a trigger. A discussion of the trigger will be presented in a later section. In a three-gap RPC system, spacers would be staggered in such a way as to allow clear passage of a muon in at least two of the three gas gaps.

The laminated plates consist of the resistive plastic coated on one side with a thin layer of graphite paint, commonly known as Aquadag. This coating serves as an electrically transparent high voltage electrode. A thin insulating mylar sheet with pick-up strips of aluminum or copper deposited on one side (available commercially) is bonded to the Aquadag-coated side of the resistive plastic sheet. Thus the high voltage electrode is insulated from the pick-up strips by the thickness of the mylar. A rigid low-density foam board (Last-A-Foam, density $0.029 \mathrm{~g} / \mathrm{cm}^{3}$ ) is used as a mechanical stiffener as well as a dielectric for the transmission line formed by the 
pick-up strips and a thin foil ground plane placed on the opposite side of the foam. The foam is glued to the mylar and a thin metal foil of aluminum is glued on the opposite side. For $1.3 \mathrm{~cm}$ strips, such as those used in the bend plane in the barrel chambers, $5 \mathrm{~mm}$ thick foam is used to form a $50 \mathrm{ohm}$ transmission line for each strip. This laminate process is repeated to form the four plates necessary for a two-layer RPC. Once the four plates are glued together with the spacers the laminate is quite rigid. For example, a single layer $1.2 \mathrm{~m} \times 2.4 \mathrm{~m}$ RPC supported at its four corners sags about $1 \mathrm{~cm}$ at its center. A double layer RPC is expected sag even less.

\section{Precision requirements}

There are no precision requirements necessary for the RPC assembly. Standard tolerances on thicknesses of plastic sheet and other commercially available laminate materials are sufficient for the proper operation of the RPCs. The insulating disk spacers can be turned out using computerized machining with the necessary height tolerance of $\pm 100 \mu \mathrm{m}( \pm 4$ mils) without difficulty. Pick-up strips can be laid out by commercial methods provided by the manufacturer of metallized mylars. Standard manufacturing processes are capable of providing the necessary dimensional tolerance of $\pm 100 \mu \mathrm{m}$.

Table 5.1 lists the breakdown of radiation lengths for the various materials in a three-gap RPC. A three-gap RPC of this construction has about $1.7 \%$ of a radiation length or about $0.55 \%$ per RPC layer. 
Table 5.1

GEM RPC Radiation Lengths

Areal Mass: $\quad$ projected thickness $=0.55 \% \mathrm{X}_{\mathrm{o}} /$ layer

Material Thickness Rad. Length (cm) \% Rad. Length

$\begin{array}{llll}\text { Al } & 0.005^{\prime \prime} & 8.9 & 0.14 \\ \text { Foa.n } & 5 \mathrm{~mm} & 900 & 0.03 \\ \text { Mylar } & 0.005^{\prime \prime} & 28.7 & 0.04 \\ \text { ABS plastic } & 0.020^{\prime \prime} & 34.4 & 0.15 \\ \text { Gas } & 2 \mathrm{~mm} & \text { Large } & 0 \\ \text { ABS plastic } & 0.020^{\prime \prime} & 34.4 & 0.15 \\ \text { Mylar } & 0.005^{\prime \prime} & 28.7 & 0.04 \\ \text { Foam } & 5 \mathrm{~mm} & 900 & 0.03 \\ \text { Al } & 0.001^{\prime \prime} & 8.9 & 0.03 \\ \text { Foam } & 5 \mathrm{~mm} & 900 & 0.03 \\ \text { Mylar } & 0.005^{\prime \prime} & 28.7 & 0.04 \\ \text { ABS plastic } & 0.020^{\prime \prime} & 34.4 & 0.15 \\ \text { Gas } & 2 \mathrm{~mm} & \text { Large } & 0 \\ \text { ABS plastic } & 0.020^{\prime \prime} & 34.4 & 0.15 \\ \text { Mylar } & 0.005^{\prime \prime} & 28.7 & 0.04 \\ \text { Foanh } & 5 \mathrm{~mm} & 900 & 0.03 \\ \text { Al } & 0.001^{\prime \prime} & 8.9 & 0.03 \\ \text { Foam } & 5 \mathrm{~mm} & 900 & 0.03 \\ \text { Mylar } & 0.005^{\prime \prime} & 28.7 & 0.04 \\ \text { ABS plastic } & 0.020^{\prime \prime} & 34.4 & 0.15 \\ \text { Gas } & 2 \mathrm{~mm} & \text { Large } & 0 \\ \text { ABS plastic } & 0.020^{\prime \prime} & 34.4 & 0.15 \\ \text { Mylar } & 0.005^{\prime \prime} & 28.7 & 0.04 \\ \text { Foam } & 5 \mathrm{~mm} & 900 & 0.03 \\ \text { Al } & 0.005^{\prime \prime} & 8.9 & 0.14\end{array}$

Total:

$1.66 \%$

Tables 5.2 and 5.3, GEM RPC Barrel Specifications and GEM RPC End-cap Specifications, contains, among many other items, the detailed breakdown of the weights. We have assumed $0.020^{\prime \prime}(500 \mu \mathrm{m})$ thick ABS plastic sheet. Note that from Table 5.2 one can calculate that the weights of the individual barrel chambers are 99 $\mathrm{lbs}, 120 \mathrm{lbs}$, and $227 \mathrm{lbs}$ for nominal radii of $4.0,6.0$, and $8.0 \mathrm{~m}$ (inner, middle and outer super-layers), respectively. Hence any chamber can be easily handled by four technicians (one for each corner) without the use of cranes or special hoisting apparatus. 
GEM RPC Barrel Specifications

Nominal Radius (m):

\# of chambers

\# of RPC layers/chamber

Width $(\mathrm{cm})$

Length $(\mathrm{cm})$

Total Length

\# Bend-plane strips:

\# Non-bend-plane strips:

Width of bend-plane strips $(\mathrm{cm})$ :

Width of non-bend-plane strips $(\mathrm{cm})$ :

Total number of channels - bend plane

Total number of channels - non-bend-plane

Total number of channels -
4.0

96

3

167.0

240.0

720.0

114

166

1.3

3.9
6.0

8.0

96

3

342.0

480.0

1440.0

253

166

1.3

8.9

Weights (lbs):

ABS Plastic:

Aluminum perimeter frame:

Connectors, brackets:

1160

2855

963

TOTAL/Super-layer

9561

11542 


\section{Table 5.3}

GEM RPC End-cap Specifications

Nominal Z(m):

\# of chambers

\# of RPC layers/chamber

Width $(\mathrm{cm})$ (16 each)

(16 each)

(16 each)

(16 each)

Length $(\mathrm{cm})$ (16 each)

(16 each)

(16 each)

(16 each)

\# Bend-plane strips (30 degrees):

\# Bend-plane strips (16 degrees):

\# Non-bend-plane strips (30 degrees):

\# Non-bend-plane strips (16 degrees):

Width of bend-plane strips

(30 degrees) $(\mathrm{cm})$ :

Width of bend-plane strips

(16 degrees) (cm):

Width of non-bend-plane strips

(16 and 30 degrees) $(\mathrm{cm})$ :

Total number of channels - bend plane

Total number of channels - non-bend plane

Total number of channels - both end-caps

Weights (lbs):

ABS Plastic:

Aluminum perimeter frame:

Cable:

Connectors, brackets:

TOTAL/Super-layer
6.0

64

3

150

140

70

75

190

180

71

76

238

186

40

26

0.6

0.44

3.8

$\begin{array}{ll}6.5 & 9.3\end{array}$

$\begin{array}{ll}6.5 & 9.3\end{array}$

71,104

6,336

77,440

16.0

64

3

370

370

180

190

470

460

180

190

583

486

40

26

0.6

$\begin{array}{ll}0.44 & 0.44\end{array}$

987

415

152

459

2013
2600

582

253

1129

4564
5046

911

381

2292

8630

\section{TOTAL WEIGHT BOTH END CAPS (TONS)}

\subsection{TONS}

The chamber widths and lengths vary $-167 \mathrm{~cm} \times 240 \mathrm{~cm}, 255 \mathrm{~cm} \times 360 \mathrm{~cm}$, and 342 $\mathrm{cm} \times 480 \mathrm{~cm}$, for inner, middle, and outer super-layer chambers, respectively in the barrel region. In the end cap region, there are two sets of chambers for coverage from theta $=9.75-16$ degrees and from 16-30 degrees. The smallest end-cap chamber has dimensions of about $70 \mathrm{~cm} \times 70 \mathrm{~cm}$, and the largest end cap chamber has dimensions of $367 \mathrm{~cm} \times 472 \mathrm{~cm}$. These dimensions are identical to the dimensions of the corresponding RDT tracking chambers. 
In summary, in the barrel there are 96 inner sector chambers, 96 middle sector chambers and 96 outer sector chambers with a total projected surface area of about 2,743 - juare meters. In the end cap there are 192 chambers grouped into 3 families of sizes distributed over the three super-layers. The total projected surface area is about 1,078 square meters.

\section{Failure tolerance}

As stated previously, the RPC is a very rugged reliable device without ser ie wires or regions of high fields. However, failures can occur. In case of a failure onr half of the double RPC would fail. Because of this possitility, we operate each RI'C gap as a separate :hamber. The loss of one RPC gap means a decrease of efficiency of $1 \%$. This is negligible from any practical point of view. Therefore we can state that the RPC system is, for practical purposes, $10 \%$ redundant and that any single failure has a negligible effect on the practical efficiency of the system.

Another way of looking at this situation is to suppose one gap fails per year. This is more than two orders of magnitude higher failure rate than is indicated by our current experience. Under this assumption one can show that the probability of two gaps failing in the same RPC chamber in ten years of operation is iess than $10 \%$.

\section{Cost Uncertainties}

The major cost uncertainty at this time is the structure cost, because the RPC may be incorporated into the drift tube structure and utilize its interface to the superstructure. In addition, the cost of machining and fabrication is somewhat uncertain. Also, electronics costs are not included in this review and estimates vary from about $\$ 11 /$ channel to about $\$ 35 /$ channel depending on the source of the electronics and whether the fabrication is in the US or overseas. Cost of materials is more certain, given the standard nature of the materials involved. Cost estimates for materials have been based on manufacturer's quotes or LLNL stock book prices for fittings, connectors, plastics, glues, etc. Details of costs have been presented in a number of reviews over the past year and can be found in various Cost Review proceedings.

In summary, it appears that a complete RPC system can be built for a less than $\$(5$ to 10$) M$. This cost can perhaps be further reduced by a simplification of the structural requirements if the RPCs are incorporated in the drift tube support system.

H. Summary

We summarize this report by reiterating a number of important facts about RPCs: 
A. They are the only Muon System technology currently made by industry. The standard sized RPC is one meter by two meters. In principle sizes much larger than this can be built.

B. They have the following pulse characteristics:

1. Pulse height about 0.5 volts without amplification into $50 \mathrm{ohms}$.

2. Puise rise time less than 5 nanoseconds.

3. Pulse width about 10 nanoseconds.

4. Measured rise time jitter less than 1.4 nanoseconds (no other Muon System technology has this speed).

C. Lifetime is greater than $16.4 \times 10^{7}$ pulses per square centimeter with no loss of efficiency within the $3 \%$ measuring error. This is equivalent to 8.2 standard SSC years (at luminosity $10^{33}$ and $2 \times 10^{7}$ seconds per year - the expected neutron flux is $2 \mathrm{~Hz}$ per square centimeter.)

D. Commercial RPCs have demonstrated essentially $100 \%$ efficiency at about $100 \mathrm{~Hz}$ per square centimeter using standard Bakelite resistive plates.

E. RPC prototypes built with new lower resistivity materials have demonstrated counting rates of about $20,000 \mathrm{~Hz}$ per square centimeter for short periods of time - in agreement with theoretical models based on bulk resistivity alone - prior to an observed increase in bulk resistivity that reduces the counting rate capability with a time constant on the order of hours. This indicates that with the proper choice of materials, i.e., with materials that exhibit stable resistive properties, we can expect RPCs with rate capabilities a factor of 1-100 higher, with an added increase in rate capability achievable by utilizing thinner materidls.

F. A number of candidate plastics have been identified and are under study, some with bulk resistivities of about $10^{9} \mathrm{ohm}-\mathrm{cm}$, which is about a factor of 100 lower than the ABS plastics currently used.

G. Refinement of the RPC design has led to an extremely low mass system with minimal muon scattering cross section of about $0.6 \% \mathrm{X}_{0} /$ layer.

H. A series of cost reviews carried out over the past year indicate that the cost of the complete RPC system for GEM is about $\$ 5 \mathrm{M}$ including the cost of the electronics.

Based on the R\&D we have performed, along with a detailed analysis of the cost of constructing a full RPC trigger system for GEM, we feel that the combination 
of pressurized duift tubes (PDTs) for precision tracking coupled to RPCs for fast Level 1 triggering has the least risk, the highest muon resolution, the best beam crossing timing accuracy, and the lowest cost of all competing technologies.

\section{General References}

1. G. Battistoni, et al., "Plastic Spark Counters with PVC Electrodes," Nucl. Inst. Meth. A270, p. 190, 1988.

2. R. Cardarelli, et al., "Progress in Resistive Plate Counters," Nud. Inst. Meth. A263, p. 20, 1988.

3. M. Della Negra, et al, "Study of Muon Triggers and Momentum Reconstruction in a Strong Magnetic Field for a Muon Detector at LHC," CERN/DRDC/90-36 DRDC/P7, August 30, 1990.

4. M. Anelli, et al., "Glass Electrode Spark Counters," Nucl. Inst. Meth. A300, p. $572,1991$.

5. "An Expression of Interest to Construct a Major SSC Detector," B. Barish, W. Willis, Co-spokespersons, pps. 3-38 - 3-40, July, 1991, unpublished.

6. C. Bacci, et al., "Muon Tracking and Hadron Punchthrough Measurements using Resistive Plate Chambers," Preprint ROM2F/91/18, July 22, 1991.

7. A. Bohrer, et al., "Status Report of the RD5 Experiment," CERN/DRDC/91-53, January 13, 1992.

8. M. Widgoff, et al., "Resistive Plate Chamber Technology Review" GEM-TN92-206, October 6, 1992.

J. Figure Captions

5.1. Sketch of Italian (single layer design) Resistive Plate Chamber.

5.2. Equivalent circuit of the RPC used in SPICE to predict the operating characteristics of the RPC. The RPC is modeled as a capacitor anci a resistor for each plate coupled via a capacitor, corresponding to the gas gap. The calculated RPC characteristics include recovery time, which is directly correlated to the saturated counting rate capability of the RPC.

5.3. SPICE calculation results for different RPC recovery times. In this figure we compare a number of different materials: Bakelite, glass, and plastics.

5.4. Comparison of SPICE calculated RPC rate capability with measured rates for a number of different RPCs. The RPCs plotted are, in order of increasing rate, the MIT glass, the LLNL glass, the Italian Bakelite, the Abstat-M310, 
and the MiTech-411. The linear fit to the points is also indicated. The rate capability is defined as the inverse of the half-height time of the curves in Fig. 5.3.

5.5. Schematic layout of materials in the proposed two layer RPC for the GEM Muon System. See Table 5.1 for the corresponding radiation length fractions. 


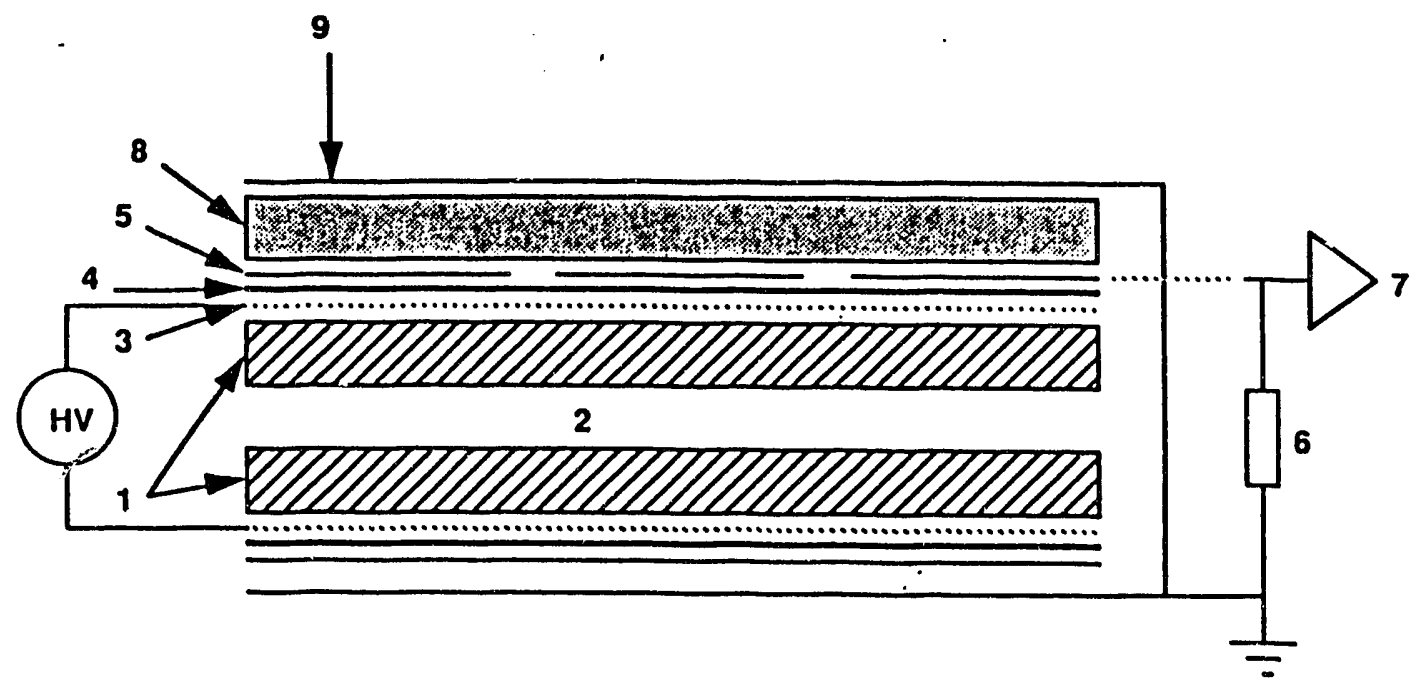

1 Bakelite plate

2 gas

3 graphite coating

4 Insulating foil

5 aluminum strips

6 line termination

7 discriminator

8 foam

9 aluminum ground plane

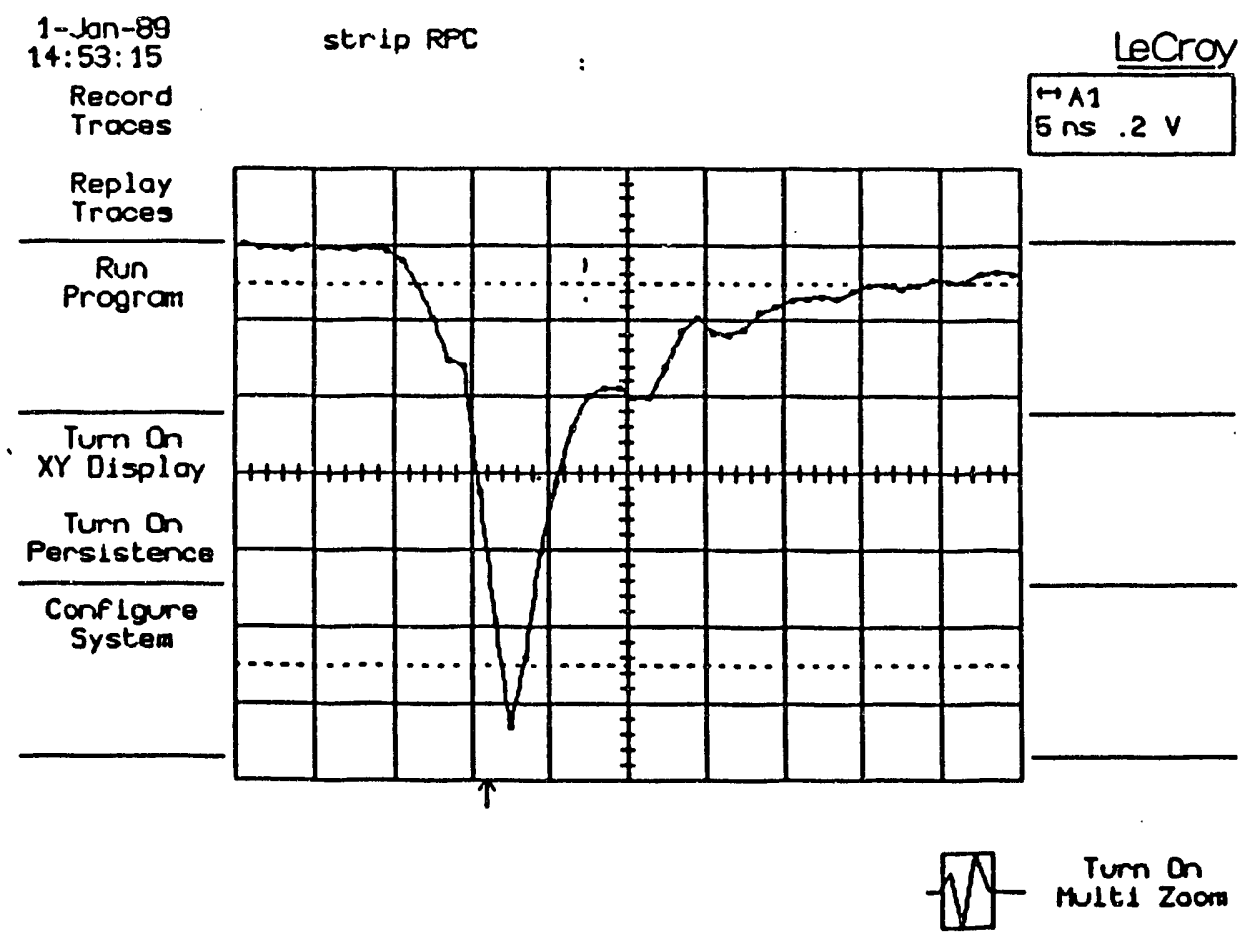

C

Fig. 5.1 

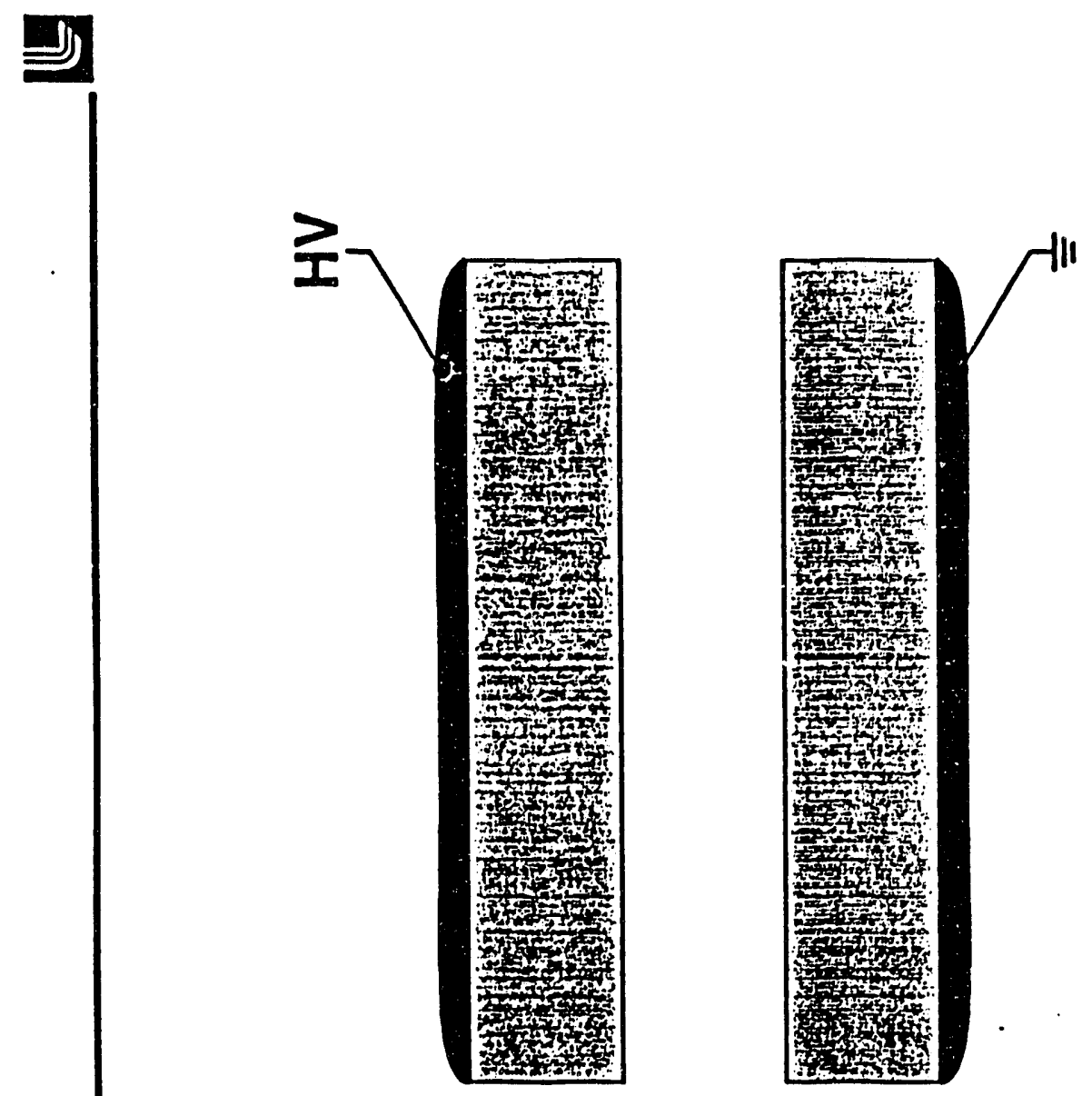

ก

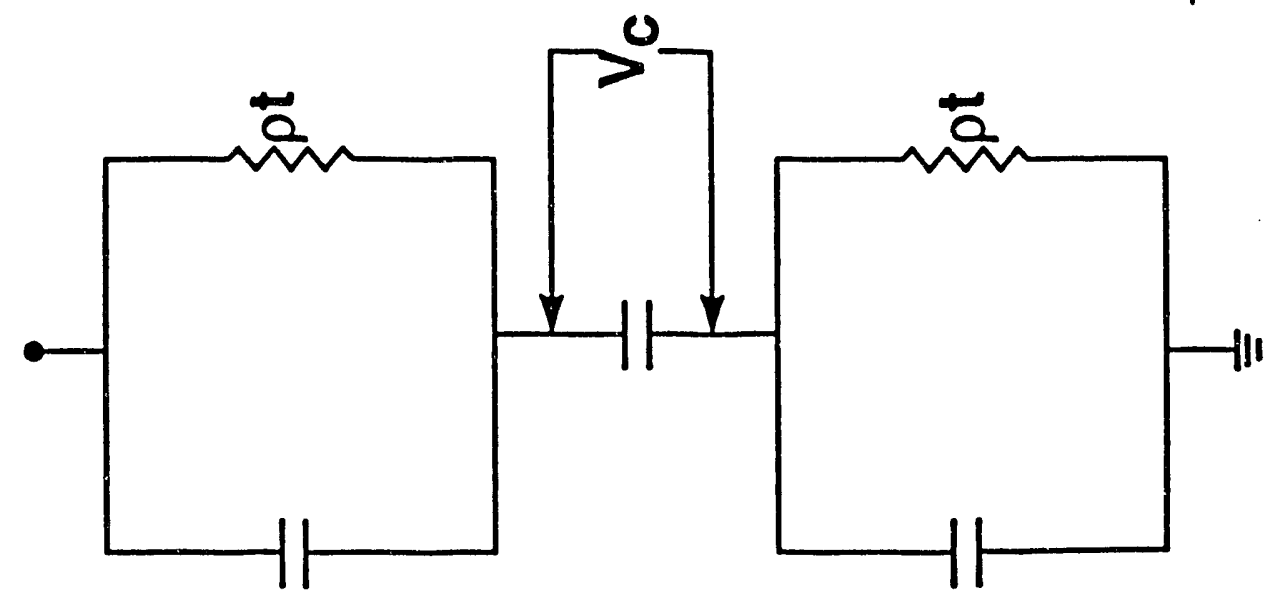



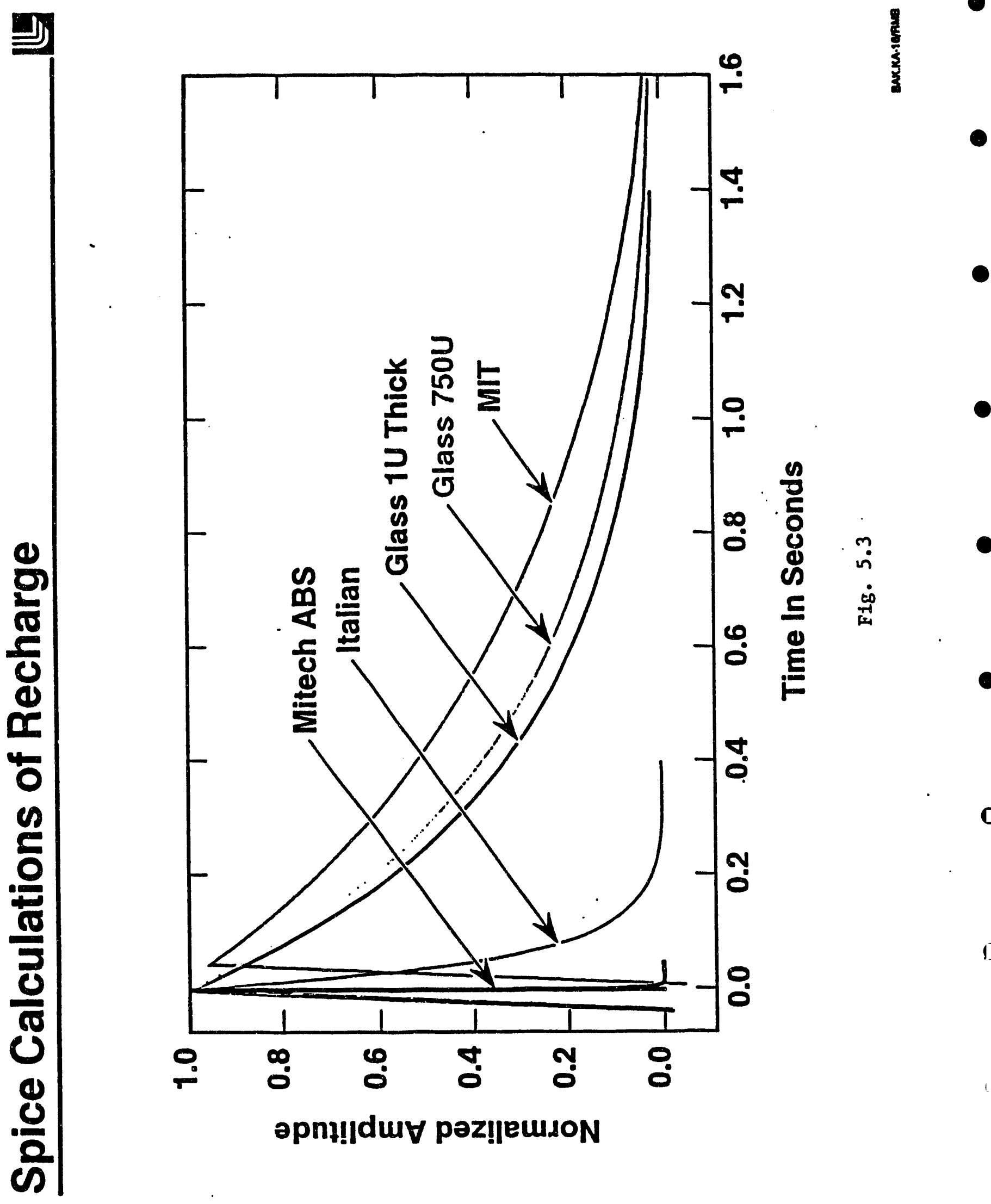

0 

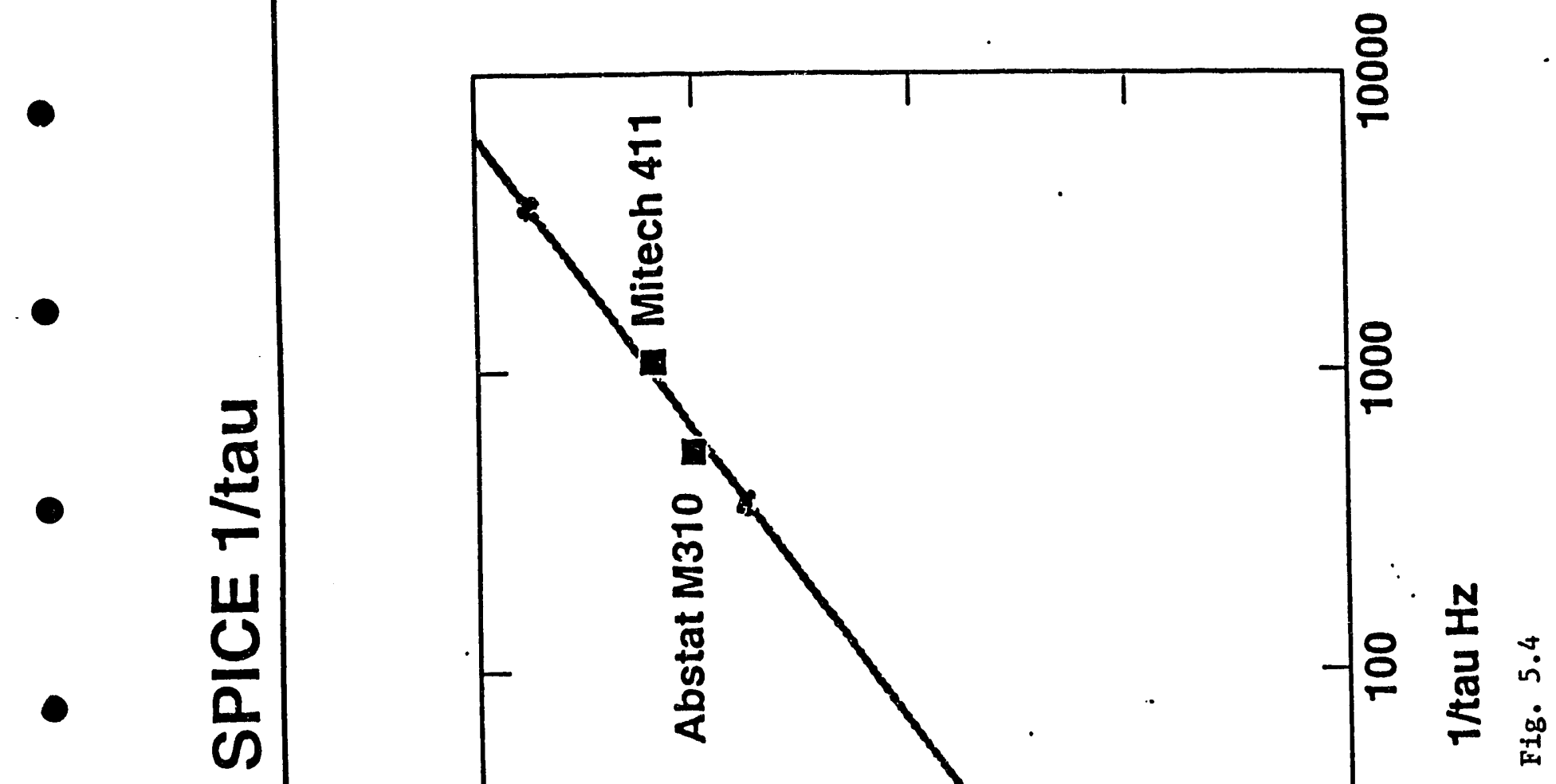

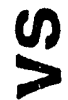

(1)

$+$

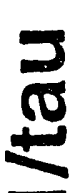

U

$\frac{0}{0}$

\%

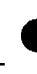

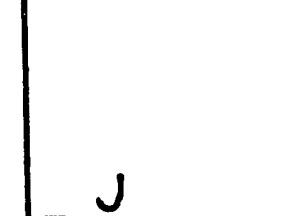

$\frac{\text { D }}{\text { के }}$

ఫ్ర

त्ञ

$\frac{1}{2}$

$-\frac{2}{2}$

ะั)

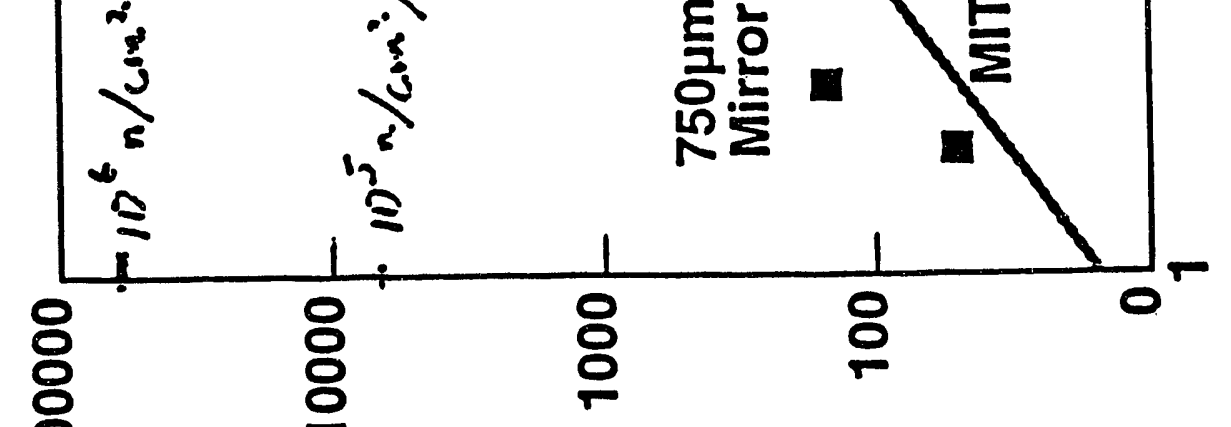

ஸึ 


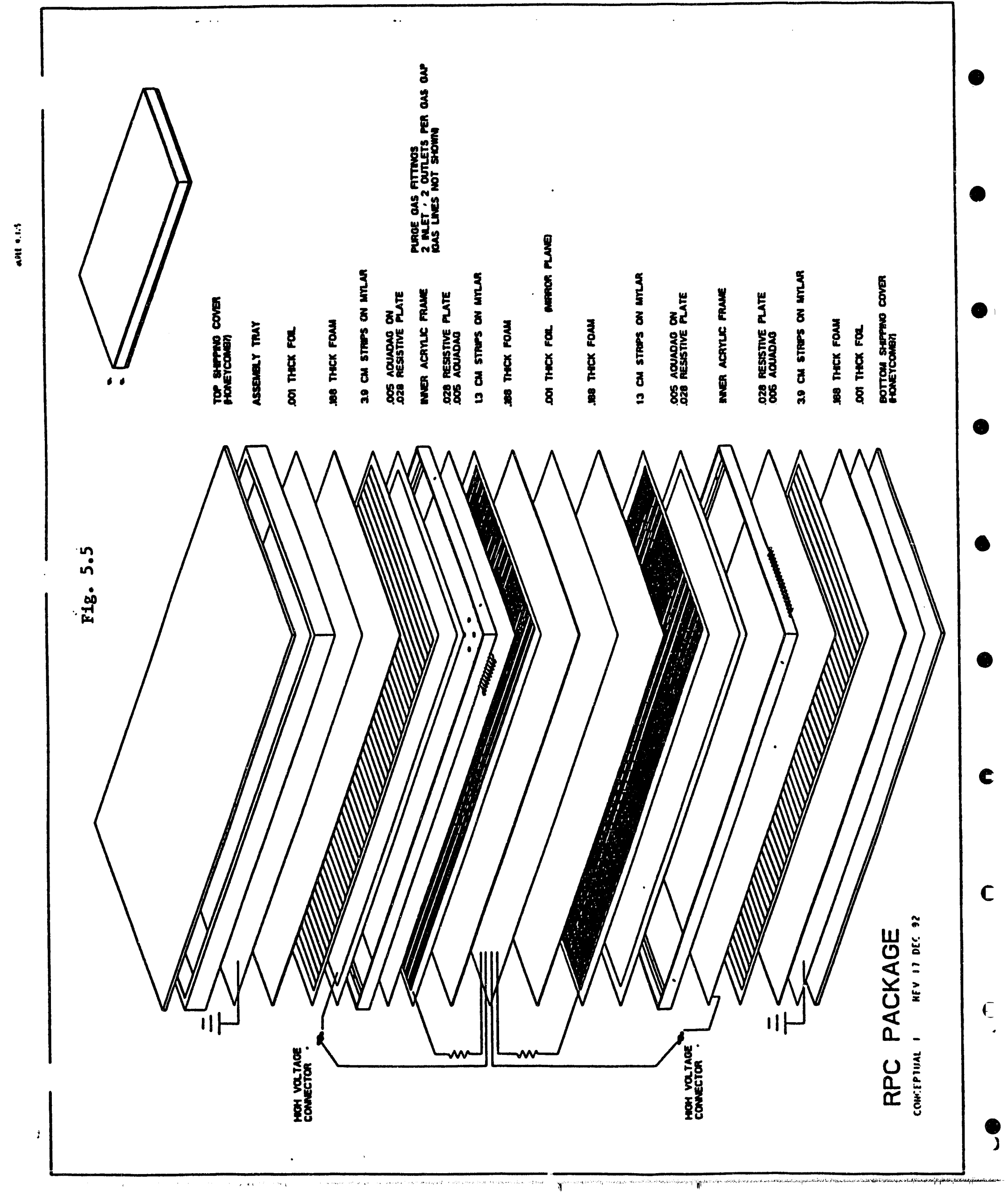




\section{(6.0) R\&D Program}

An active R\&D program was carried out to determine the properties the RDT and RPC technologies. The program had two facets: (1) to understand the basic characteristics of the technologies, and (2) to certify the design concepts by building and operating full-scale prototypes. In the course of this program a variety of chambers were constructed - and tested with cosmic rays at the SSCL.

\section{(6.1) Tests of Large Scale RDT Prototypes}

Three large scale prototypes based on drift technology were constructed and tested at TTR:

- Dubna RDT Chamber.

- MSU RDT Chamber

- MIT LSDT Chamber.

These prototypes were designed to answer the crucial questions related to a performance of large detectors based on drift technology and to justify that the performance claimed in the baseline is achievable. It should be also noted that the tests are still underway and the results presented here must be treated as preliminary - more data are expected to follow.

\section{Description of Prototypes}

\section{Dubna RDT Chamber:}

This prototype consists of four staggered by half tube diameter planes of stainless steal tubes ( $3 \mathrm{~cm}$ diameter $4 \mathrm{~m}$ long tubes with wall thickness of $.5 \mathrm{~mm}$ ); Fig. 6.1.1(a). Actually, two separate chambers $(4 \mathrm{~m}$ long and $0.5 \mathrm{~m}$ wide) are precisely joined to make the $1 \mathrm{~m}$ wide prototype. Each of these halves has 64 tubes so that the prototype consists of 128 tubes. Tubes are glued to each other without any special tolerances (however; certain stiffness of the structure is absolutely necessary to assure rigid wire support). Wire alignment was achieved by using high precision combs during chamber manufacturing (these combs, rather than the tube end-plugs, were determining wire position while they were soldered, as shown in Fig. 6.1.2; details may be found elsewhere [1]). Anode wires $(50 \mu \mathrm{m}$, Au plated $W)$ are stretched with tension of $300 \mathrm{~g}$. Signals are amplified by low noise fast amplifiers mounted directly on tubes (the amplifiers allowed to set a threshold as low as $1 \mathrm{~mA}$, signal rise time being around $10 \mathrm{~ns}$ ), then, after going through $\sim 16 \mathrm{~m}$ long coaxial cables, they are discriminated and after additional $30 \mathrm{~m}$ flat cables fed to 12 bit $0.2 \mathrm{~ns}$ bin width TDC's (Philips7186H) . Chamber design allowed to use gas mixtures under high pressure up to 5-8 atm. 


\section{MSU RDT Chamber:}

The MSU Prototype is a $4 \mathrm{~m}$ long $1.2 \mathrm{~m}$ wide chamber made of 4 staggered (by half tube diameter) planes of $\mathrm{Al} 3.8 \mathrm{~cm}$ diameter tubes with a wall thickness of $300 \mu \mathrm{m}$; Fig. 6.1.1(b). Total number of tubes is 128. Anode wires are the same as in Dubna RDT's, however; the alignment is achieved by a different method. The wires are fixed in the precise holes made in the chamber end-plates -- see Fig. 6.1.3. The accuracy of these end-plates was measured (Fig. 6.1.4) and found to satisfy the requirements. The tubes were glued to each other during manufacturing the prototype. This makes the prototype be very stiff. Details of the chamber design may be found elsewhere [2]. The chamber can be operated in both proportional and streamer modes. Drift times are measured by LeCroy 2277 . TDC's operated in common stop mode (1 ns bin width, $50 \mu \mathrm{s}$ depth, 16 hits per channel capability). Signals (can be amplified if the chamber operates in proportional mode) are discriminated and after not very long flat cable fed in TDC's. The chamber can operate at high pressure as well. So far, the chamber have been tested in streamer mode at $1 \mathrm{~atm}$.

\section{MIT LSDT Chamber:}

This prototype is essentially different since it is assembled of U-shaped $10 \mathrm{mil} \mathrm{Al}$ profiles so that a drift cell has a square geometry; Fig. 6.1.1(c). Although, this design is not considered any more, many of the results obtained on this chamber are independent of cell geometry and provide important information to be discussed later on. The chamber is $1 \mathrm{~m}$ wide and $4 \mathrm{~m}$ long. Four profiles make four planes of square tubes with $2.5 \mathrm{~cm}$ cell size. Planes are staggered by half cell size. $100 \mu \mathrm{m}$ wires (Au plated W, $1.2 \mathrm{~kg}$ tension) are supported each $2 \mathrm{~m}$ by precisely machined Mycalax bridges. Details of the design are in [3]. The chamber was tested in streamer mode only. The signals (no amplification) are shaped by discriminators mounted on the chamber and drift time digitization is performed by LeCroy 2277 TDC operated in common start mode ( $200 \mathrm{ft}$ of flat cable between TDC and discriminators). The anode wires are connected in pairs at the rear end so that signal propagates in both directions and measured twice. The time difference provides the coordinate along the wire. Only 64 wires were actually instrumented with electronics.

\section{Single layer resolution}

The single layer resolution is a combination of an intrinsic resolution (determined by ionization density, diffusion and mode of operation: streamer vs. proportional), uniformity of gas through out the volume of chamber, alignment of wires in the chamber, wire droop (due to gravity and electrostatic forces) and, also, may include some multiple scattering.

Enormous amount of studies (both calculations and tests) devoted to high precision drift detectors have been done during recent decades. The intrinsic spatial resolution under $100 \mu \mathrm{m}$ (even with rather simple electronics and at $1 \mathrm{~atm}$ gas pressure) has been demonstrated in many different drift detectors. Some of these results are shown 
in Fig.6.1.5 [4]. Also, it should be noted that recent calculations have shown that the spatial resolution in proportional mode should not be much worse than that in streamer mode (degradation occurs only in the vicinity of the anode wire - Fig.6.1.6 [5]).

Thus, the goal of R\&D was to demonstrate that one can keep the good drift readout resolution in chambers of the proposed scale. The following is a summary of the most important results obtained by now.

\section{Dubna RDT's}

Just one half of the prototype has been analyzed by the time being, i.e. 64 wires. Fig.7 shows resolution achieved in a even smaller part of the prototype ( $6 * 4$ wires) during preliminary tests outside of TTR. One can see that the resolution of $100 \mu \mathrm{m}$ has been achieved both in proportional and streamer modes.

The two tables given in Fig.6.1.7 shows that a certain misplacement of planes has been found and, therefore, can be corrected for (it is absolutely reproducible!). The fact that the planes turned out to be misaligned is not surprising for this prototype as one can see from the way it was assembled. Fig.6.1.8 shows the residuals for 4 planes (now 32 wires per plane). One can clearly see asymmetrical tails which turned out to be a manifestation of incoherent wire misalignment. Fig.6.1.9(a) makes this fact obvious. All these observations resulted in the effort to understand if it is possible to extract geometrical corrections from the data. A standard software procedure has been formalized: first, plane-to-plane shifts should be corrected; then, individual wire shifts (see Fig.6.1.9(a) and Fig.6.1.9(b)) are to be taken into account; after that, one can look at plane rotations and correct on them (rotations are easily can be found by looking at residuals vs. track position along the wires -- Fig.6.1.10); and at the next and last step some small residual rotations of individual wires may be reconstructed. After this procedure, one gets number of small geometrical corrections which after having been applied make possible to reach the limit of intrinsic resolution, but over the large area of a chamber. The resolution of $85 \propto \mathrm{m}$ with obvious bumps and humps have transformed in symmetrical peak with $s=67 \mu \mathrm{m}$ (at 1 atm!). To make sure that these corrections are really geometrical, they have been applied for analysis of data obtained many days later (different gas mixture, pressure also varied from 1 to 5 atm). Fig.6.1.11 shows that resolution of $50 \mu \mathrm{m}$ has bieen easily achieved at $4.8 \mathrm{~atm}$ and no misalignment is visible at this level. The improvement of resolution with a pressure increase is presented in Fig.6.1.12.

Further analysis in this direction is expected to be done. It should finally prove that this procedure does not result in systematic errors.

The resolution is generally uniform across the chamber (no significant variations of resolution vs angles $\theta, \varphi$ and, also, along the wires have been found). The last confirms that a signal propagates along $4 \mathrm{~m}$ wires without significant distortion. 


\section{MSU RDT's}

So far, this prototype has not been analyzed so thoroughly as the Dubna one. Obviously, to achieve a high resolution, one need to go through with calibrations. Unfortunately, this prototype does not provide a build-in calibration for electronic channels (hopefully, this problem will be overcome in the nearest future). It should be noted that despite the absence of proper calibration, the resolution for this prototype has been shown to be around $100 \mu \mathrm{m}$ (Fig.6.1.13).

However, the already available information is important and complimentary to the Dubna prototype results. The real future muon chambers are now assumed to be constructed in the way this prototype had been made. Therefore, information about accuracy of wire placement in this prototype is essential. To determine wire positions, one really does not need to have accurate calibrations of electronics and delays. These errors would make residual distributions wider, but they can not shift mean values. One can see in Fig. 6.1.13 that there is no plane shifts in this prototype. Fig.6.1.14 shows that wires are located in places they are supposed to be and Fig.6.1.15 shows that there are also no plane rotations with respect to each other. Thus, it is proven that this design has very high intrinsic mechanical accuracy.

\section{MIT LSDT's}

This prototype has demonstrated a high spatial resolution (Fig.6.1.16) and has not required any geometrical corrections on wire placement (i.e. similar results might be expected from MSU chamber after proper calibration of electronics). Fig.6.1.17 shows resolution vs. distance from a track to a wire. It is worth to show that these results are in agreement with truly intrinsic resolution which was obtained on a smaller chamber in high energy muon beam (Fig.6.1.18). One can see that resolutions close to a wire is essentially the same and determined by distance between clusters. Then, the small chamber actually had a flat resolution of $50-55 \mu \mathrm{m}$ up to $12 \mathrm{~mm}$ away from a wire (maximum drift distance). However, the large scale prototype has a limit of 70 $\propto \mathrm{m}$. When wire misalignment as measured during assembly (25 $\mu \mathrm{m} ;$ Fig.6.1.19) and $.6 \mathrm{~ns}$ intrinsic TDC time resolution (effectively $30 \mu \mathrm{m}$ contribution) are taken into account, the expected value would be $50+30+25 \sim 65 \mu \mathrm{m}$. Some degradation of the resolution beyond $10 \mathrm{~mm}$ drift distances is apparently connected with a square cell geometry.

A summary of resolution dependence on track inclinations and position along a wire is presented in Fig.6.1.20. No significant dependence has been observed.

One more interesting result is presented in Fig.6.1.21. This figure shows an achievable accuracy of track localization along the wire as calculated from time difference between signals at a connected pair of wires. One can see that this technique allows to get about $10 \mathrm{~cm}$ accuracy (the tails come from TTR coordinate 
predictions). This says that $8 \mathrm{~m}$ propagation of a signal along a wire does not result in significant signal distortions. Since this coordinate measured from the same wire, it provides a space-point measurement of a track in a single plane what significantly increases a pattern recognition capability.

\section{Conclusions:}

1. A $25 \mu \mathrm{m}$ accuracy mechanical alignment of wires within a drift chamber has been demonstrated.

2. A spatial resolution of $50-100 \mu \mathrm{m}$ is easily achievable through out the whole chamber - independent of the incident angle.

3. Non-flammable gas mixtures are available.

4. In case of unexpected misalignment there is possibly a way to find the geometrical corrections to restore the good resolution (some further studies are still needed).

5. Resolution of $10 \mathrm{~cm}$ along a wire is achievable by using a time difference technique.

\section{References:}

1. S. Movchan, in GEM TN-92-241

2. C. Bromberg, GEM TN-92-20:5

3. R. Sumner and et al., GEM TN-92-203

4. e.g. a) Filatova and et al., NIM 143 (1977) 17

b) MIT group, "Tests of a LSDT chamber at .5 TeV muon beam", to be published

c) Dubna group, (Tests of LSDT's at CERN beam presented by I.Golutvin, in GEM TN-92-218

5. A.Korytov, "One More Time about Spatial Resolution in Drift Chambers", to be published.

\section{Figure Captions:}

Fig. 6.1.1(a) Dubna Prototype Schematic.

Fig. 6.1.1(b) MSU Prototype Schematic.

Fig. 6.1.1(c) MIT Prototype Schematic.

Fig. 6.1.2 Concept of wire alignment in the Dubna Prototype: 
(a) general view -- wires are supported by precise combs;

(b) wires do not touch end pl ags during soldering.

Fig. 6.1.3 Concept of wire alignment in the MSU Prototype: precision is determined by an accurately machined manifold plate and a tube end plug.

Fig. 6.1.4 Mechanical accuracy achieved in the manifold plate.

Fig. 6.1.5(a) LSDT ?* +ial resolution [4b];

Fig. 6.1 5(b) LSDT -ratial resolution [4b] - non-flammable gas, 1 atm;

Fig. 6...j(c) Drift Readout resolution [4a];

Fig. 6.1.5(d) LSDT spatial resolution [4c] - non-flammable gas, 3 atm.

Fig. 6.1.6. MC simulation of drift readout resolution with a fixed threshold: triggering on the first electron (streamer mnde) is desirable but not necessary.

Fig. 6.1.7. First results from the Dubna Prototype: planes are misplaced but it is correctable in off-line.

Fig. 6.1.8 Dubna Prototype:

Residuals for all four planes - some asymmetry is obvious.

Fig. 6.1.9 Dubna $P_{1}$ 'otype:

(a) incoherent wire misplacements are responsible for bumps and humps of residual distribution (Fig.6.1.8);

(b) wire positions have been corrected.

Fig. 6.1.10. Dubna Prototype:

Small rotations of planes could be also corrected.

Fig. 6.1.11. Dubna Prototype:

When all found geometrical corrections have been applied, the resolution at $4-5 \mathrm{~atm}$ is about $50 \mu \mathrm{m}$. (Notice that the corrections have been found from data obtained several days before in a different gas at $1 \mathrm{~atm}$.)

Fig. 6.1.12. Dubna Prototype: Resolution vs. Pressure.

Fig. 6.1.13. MSU Prototype:

No systematic plane shifts have been discovered (mean values of residuals are of the order of $1 \mathrm{~mm}$ ).

Fig. 6.1.14. MSU Prototype:

All wires in places where they are supposed to be. 
Fig. 6.1.15. MSU Prototype: No plane rotations.

Fig. 6.1.16. MIT Prototype:

Resolution is $78 \mu \mathrm{m}$ (all planes, no geometrical corrections!).

Fig. 6.1.17. MIT Prototype:

Resolution vs distance from a wire $(\sim 70 \mu \mathrm{m}$ at plateau).

Fig. 6.1.18. MIT Prototype:

Intrinsic resolution as measured in a smaller prototype at

Fermilab [4b] $(\sim 50 \mu \mathrm{m}$ at plateau).

Fig. 6.1.19. MIT Prototype:

Wire misalignment as measured during assembly of the Prototype (RMS $25 \mu \mathrm{m}$ ).

Fig. 6.1.20(a) MIT Prototype: No wire misplacements.

Fig. 6.1.20(b) MIT Prototype:

Resolution vs coordinate along the chamber: no changes.

Fig. 6.1.20(c) MIT Prototype: Resolution vs q angle: no changes.

Fig. 6.1.20(d) MIT Prototype: Resolution vs j angle: no changes.

Fig. 6.1.21. MIT Prototype:

Resolution along a wire as obtained by time difference technique $(\mathrm{s} \sim 10 \mathrm{~cm})$. 

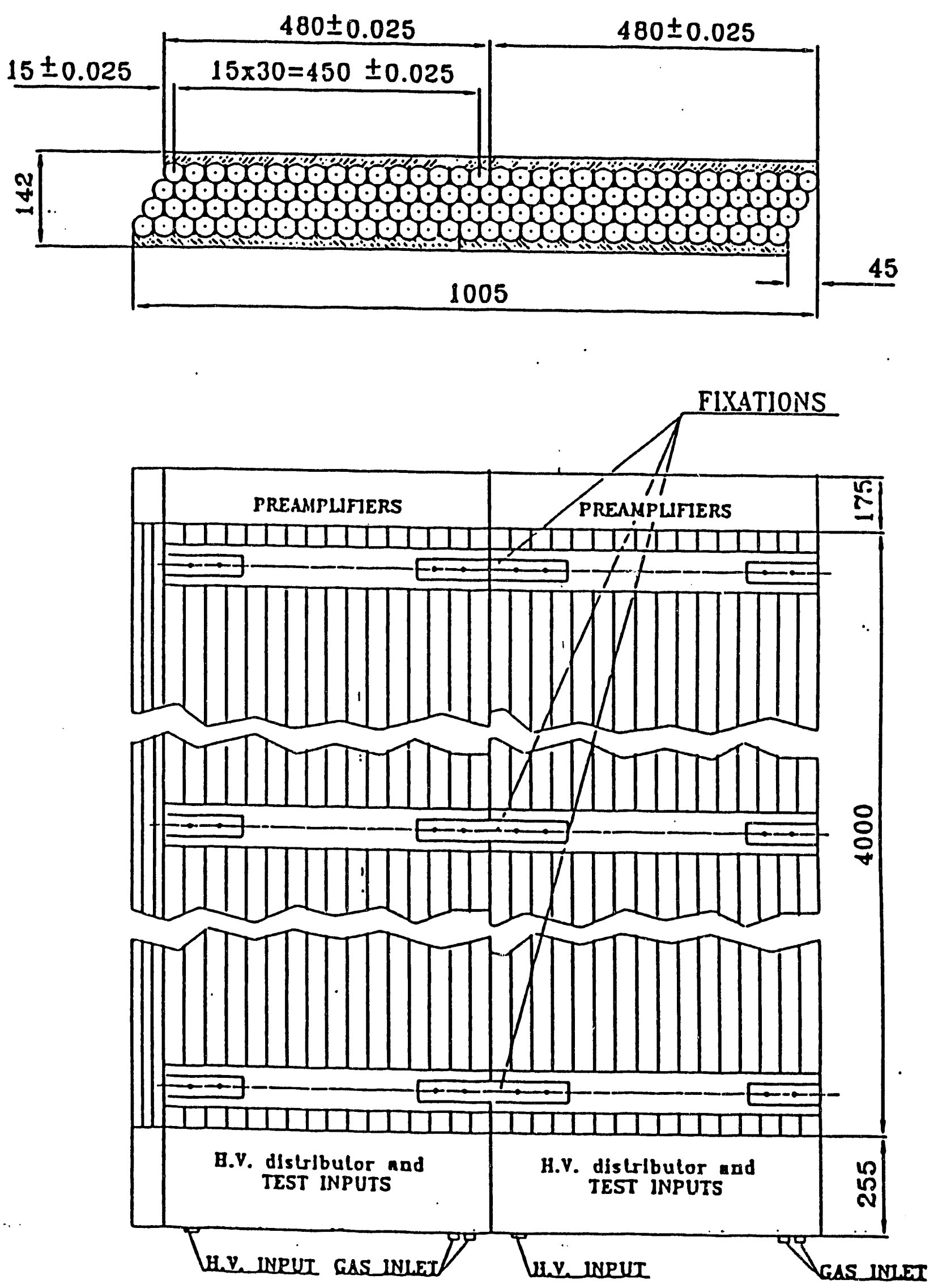

F1g. 6.1.1a 
Alignment Holes

Line of sight through each comer to far end

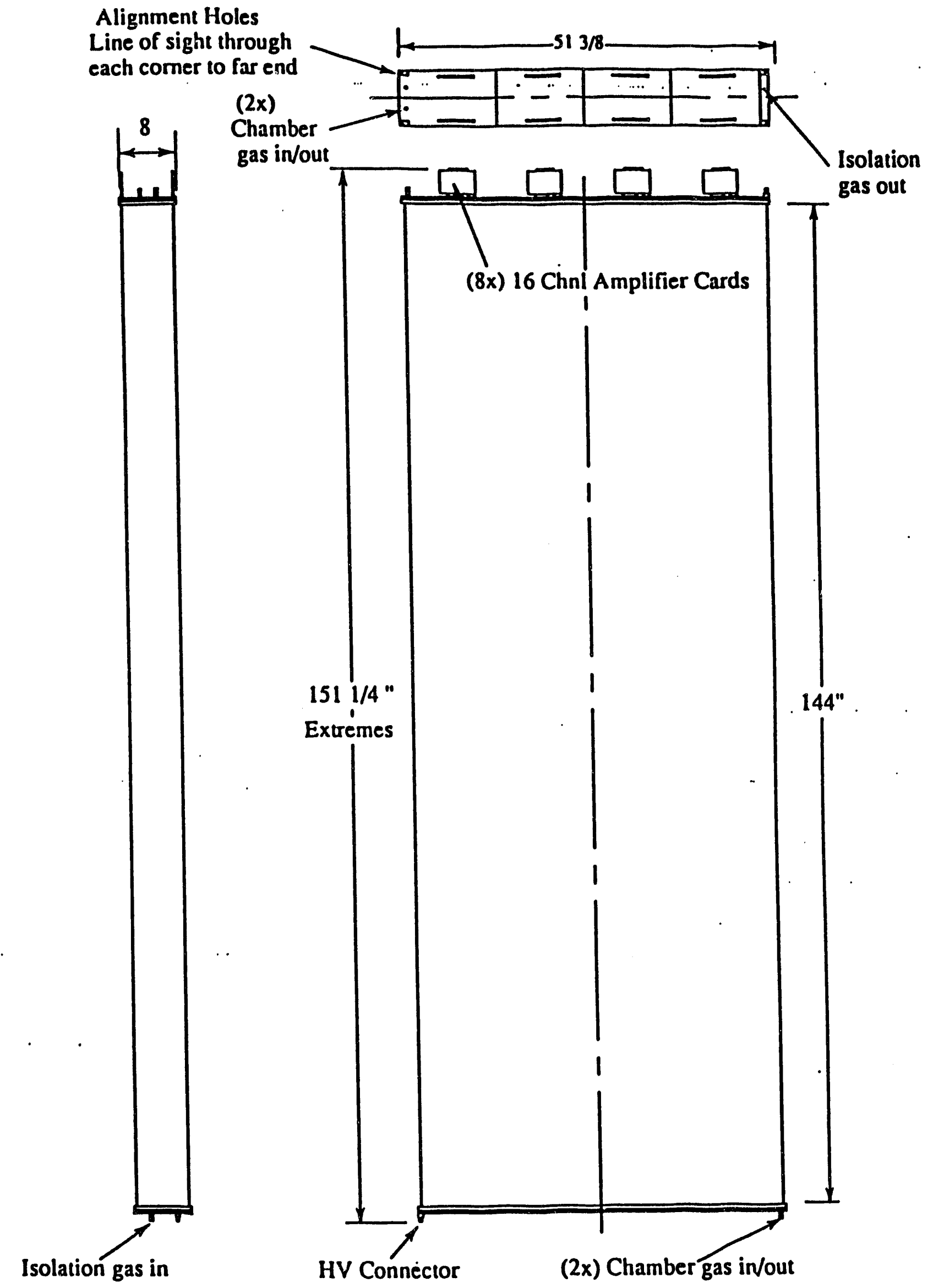

Isolation gas in

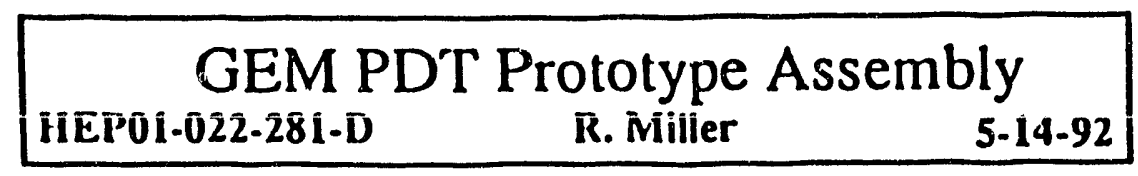

Fig. 6.1.1b 


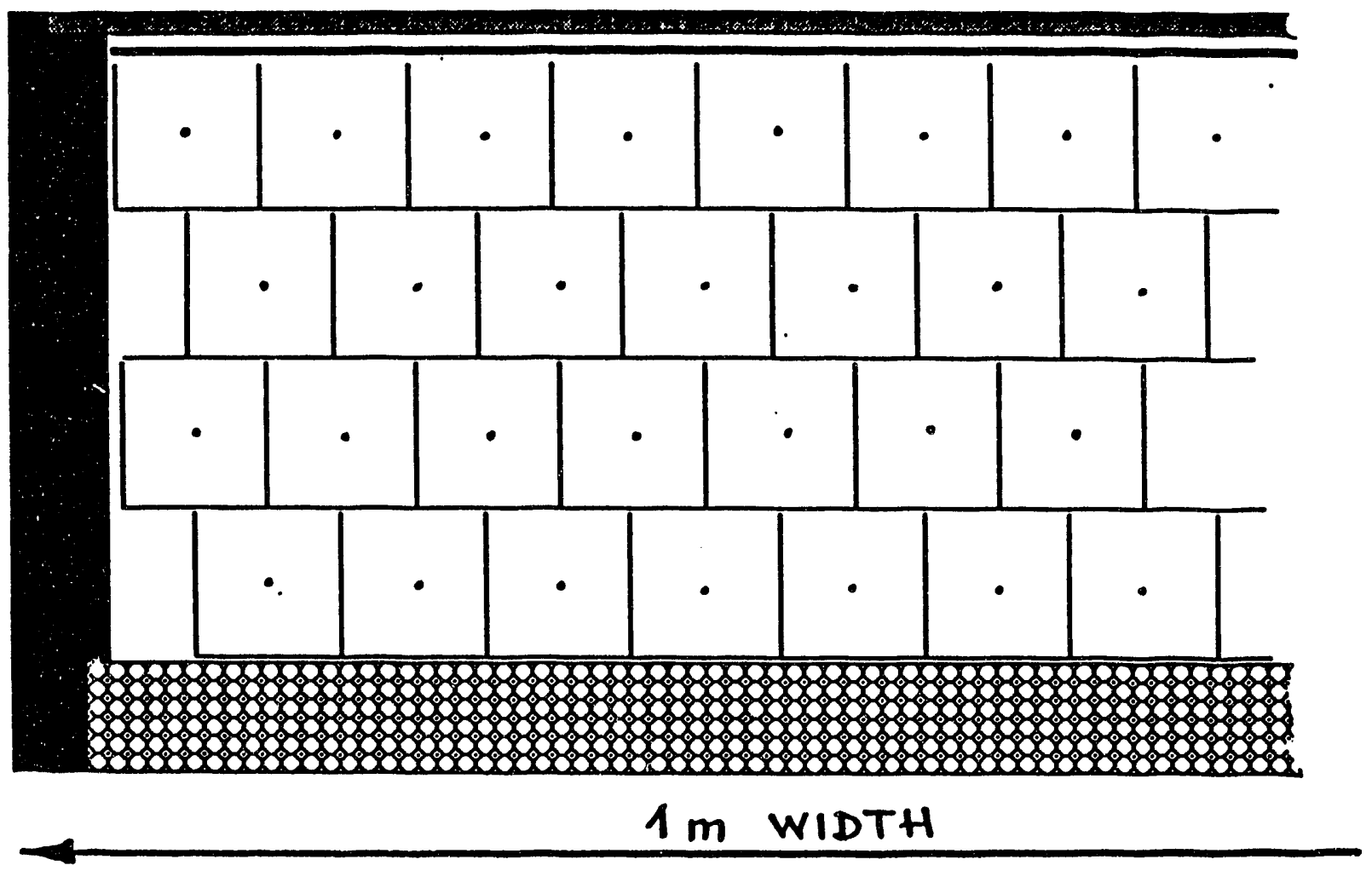

6

C

c

F1g. 6.1.1c 

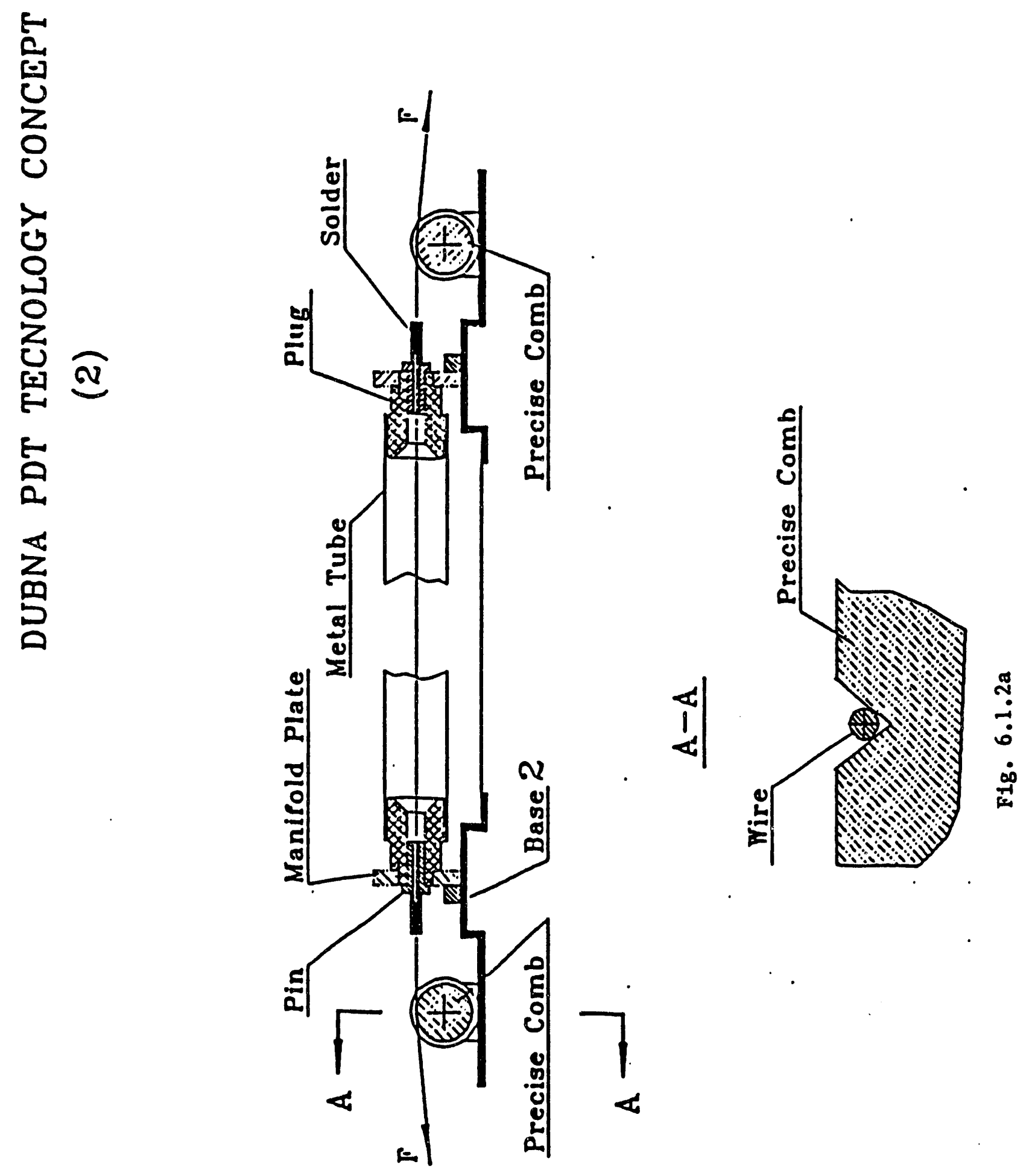


\section{DUBNA TECNOLOGY CONCEPT}

(3)

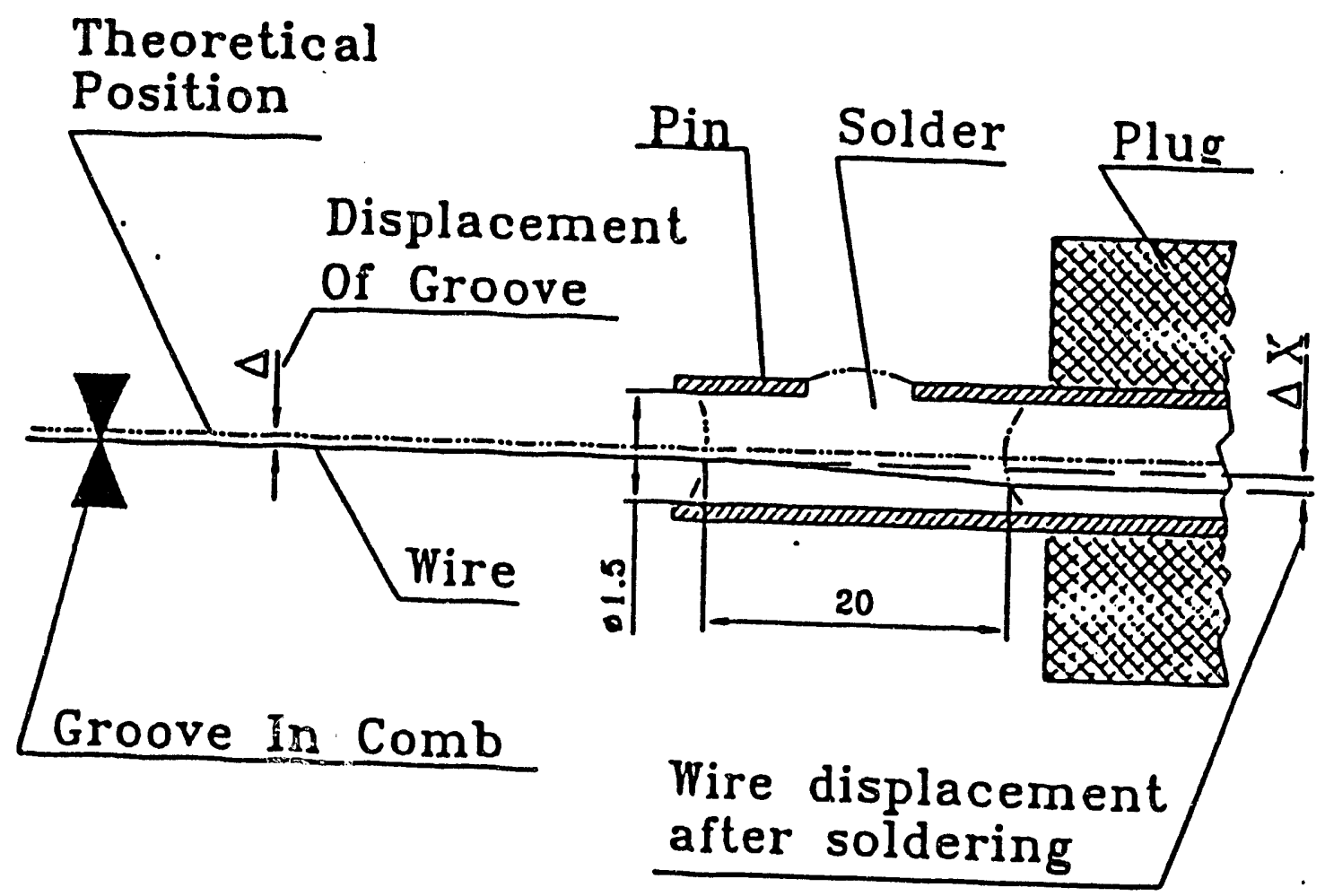

Fig. $6.1 .2 b$ 


\section{Solder.}

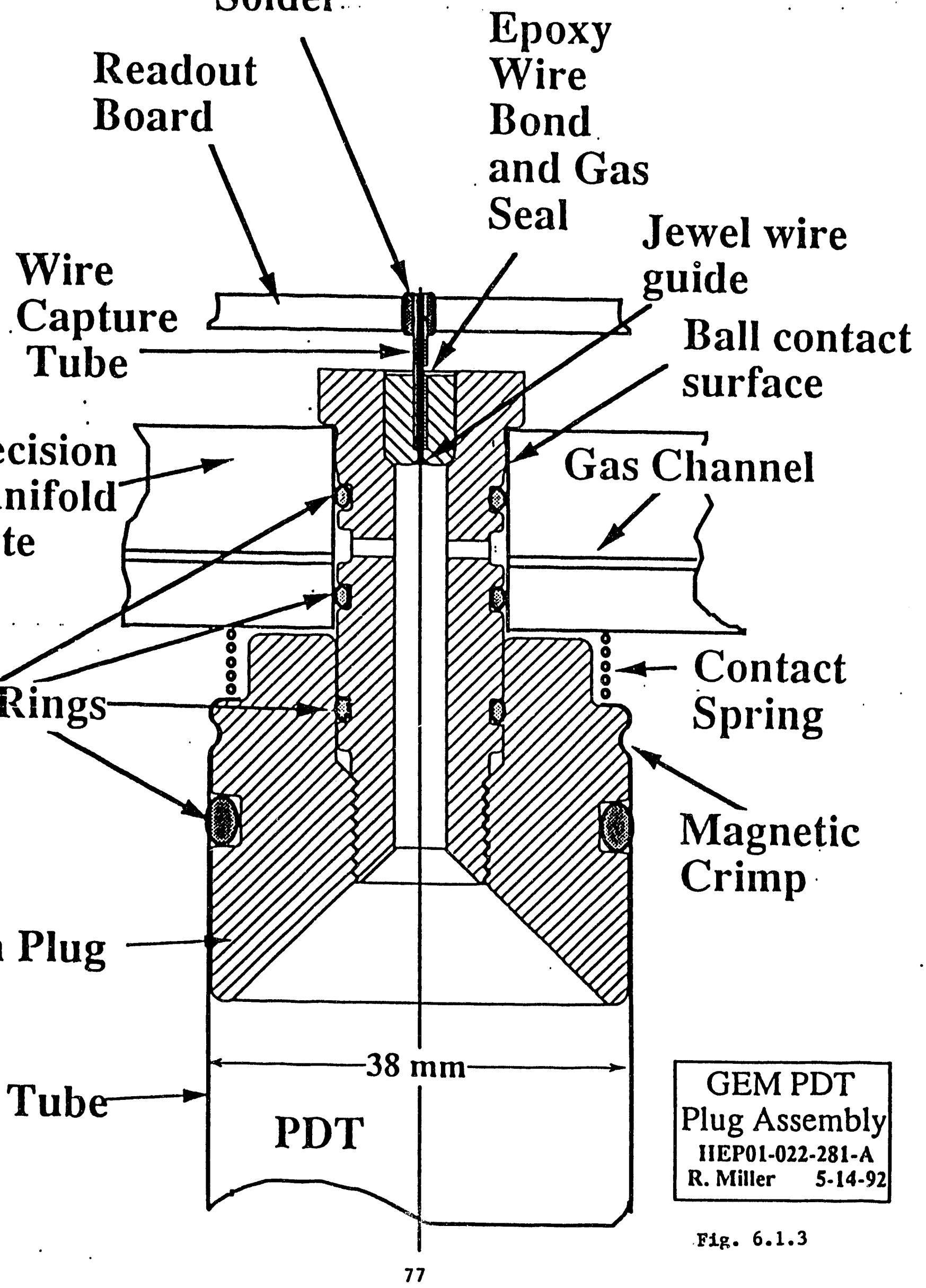



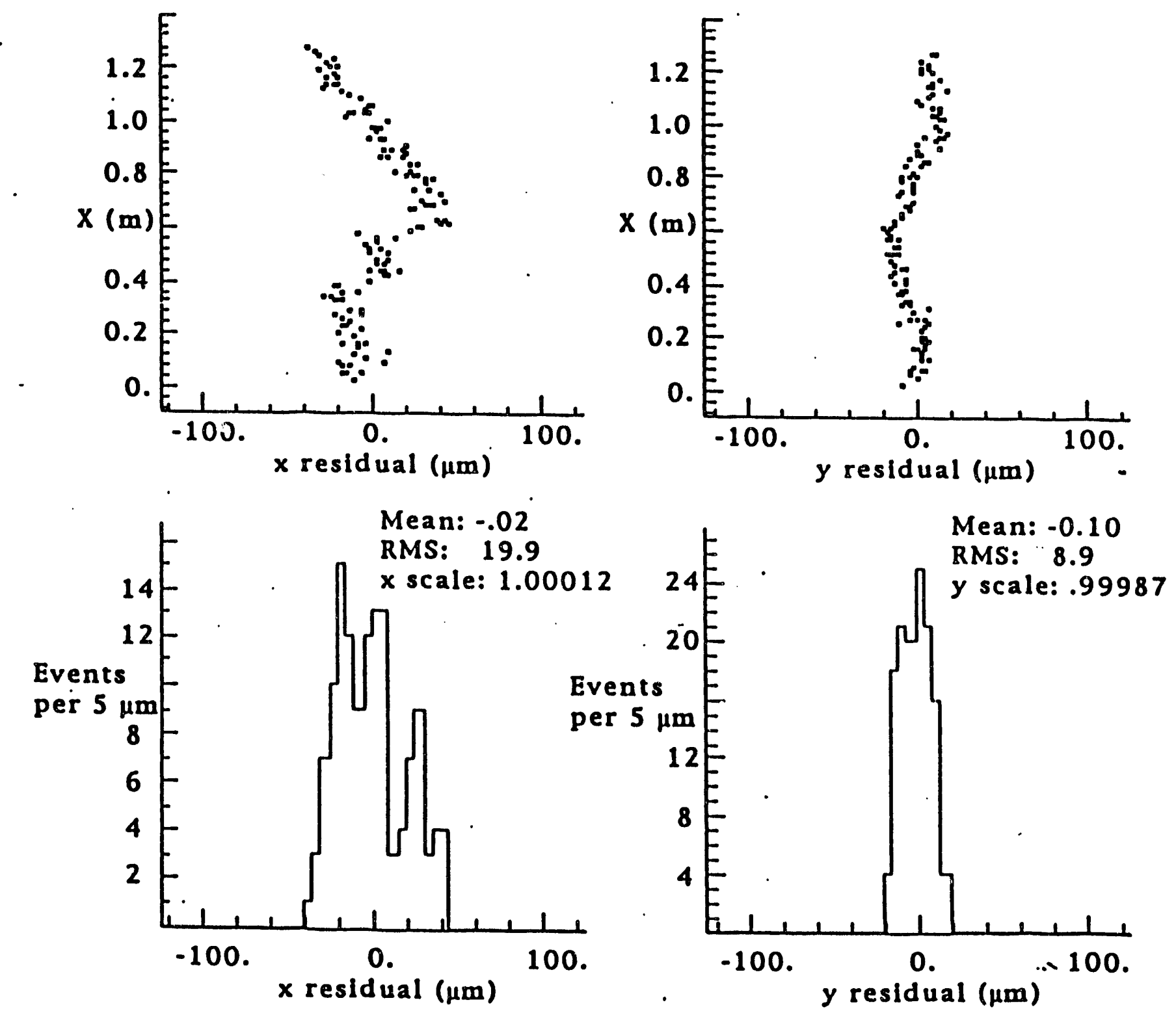

Fig. 6.1 .4 


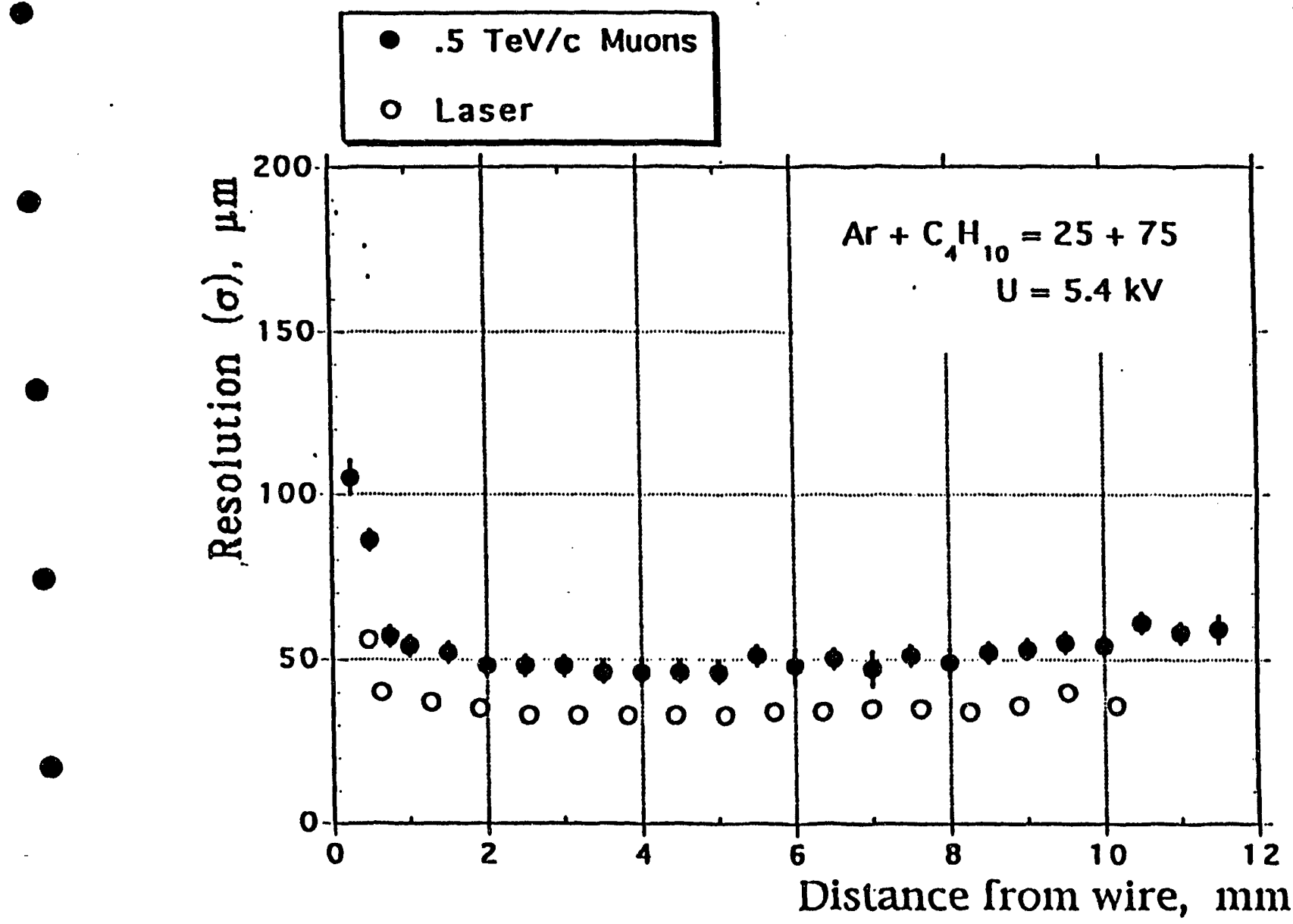

Fig. $6.1 .5 a$ 


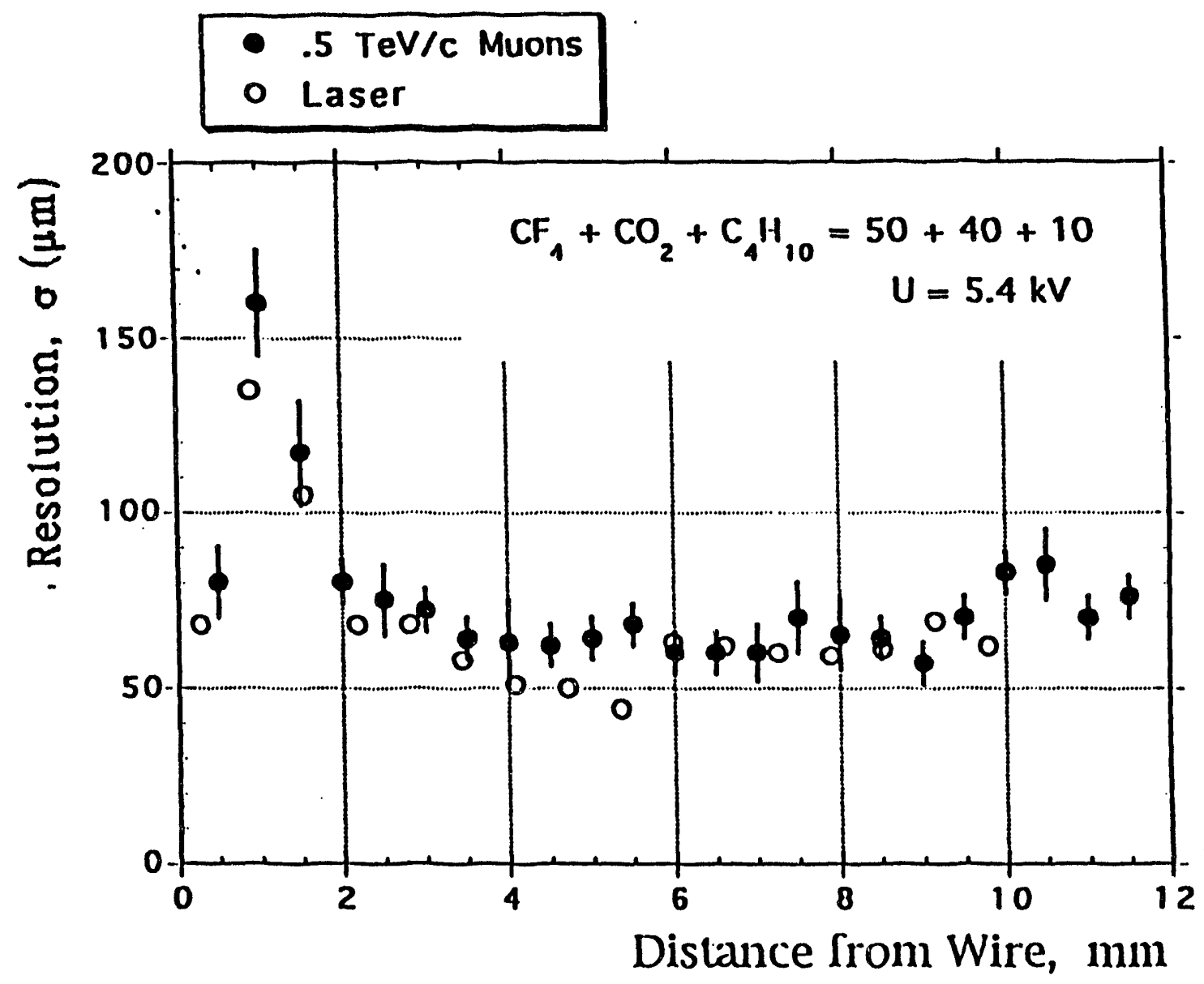

c

Fig. $6.1 .5 b$ 
NIM $143(1977) 17$

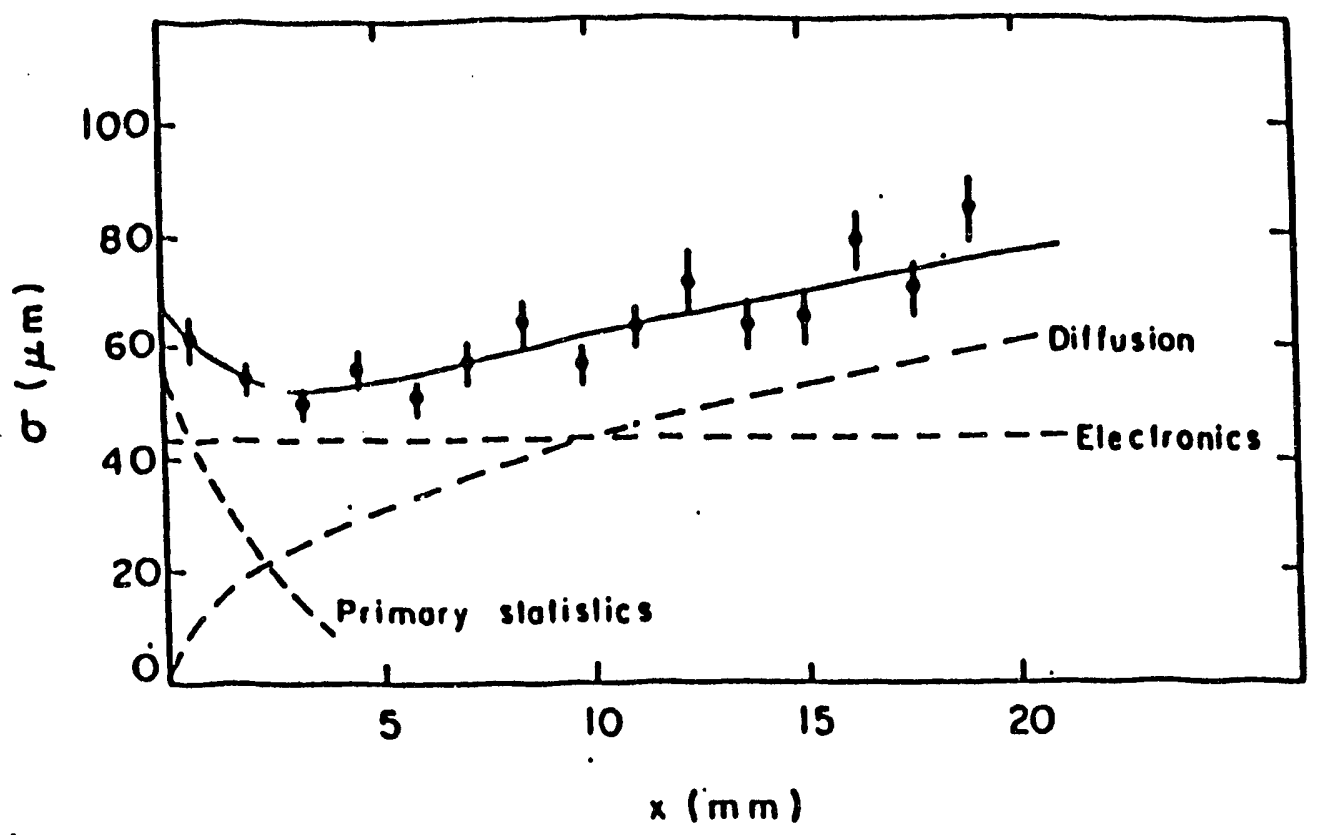

Fig. $6.1 .5 c$ 


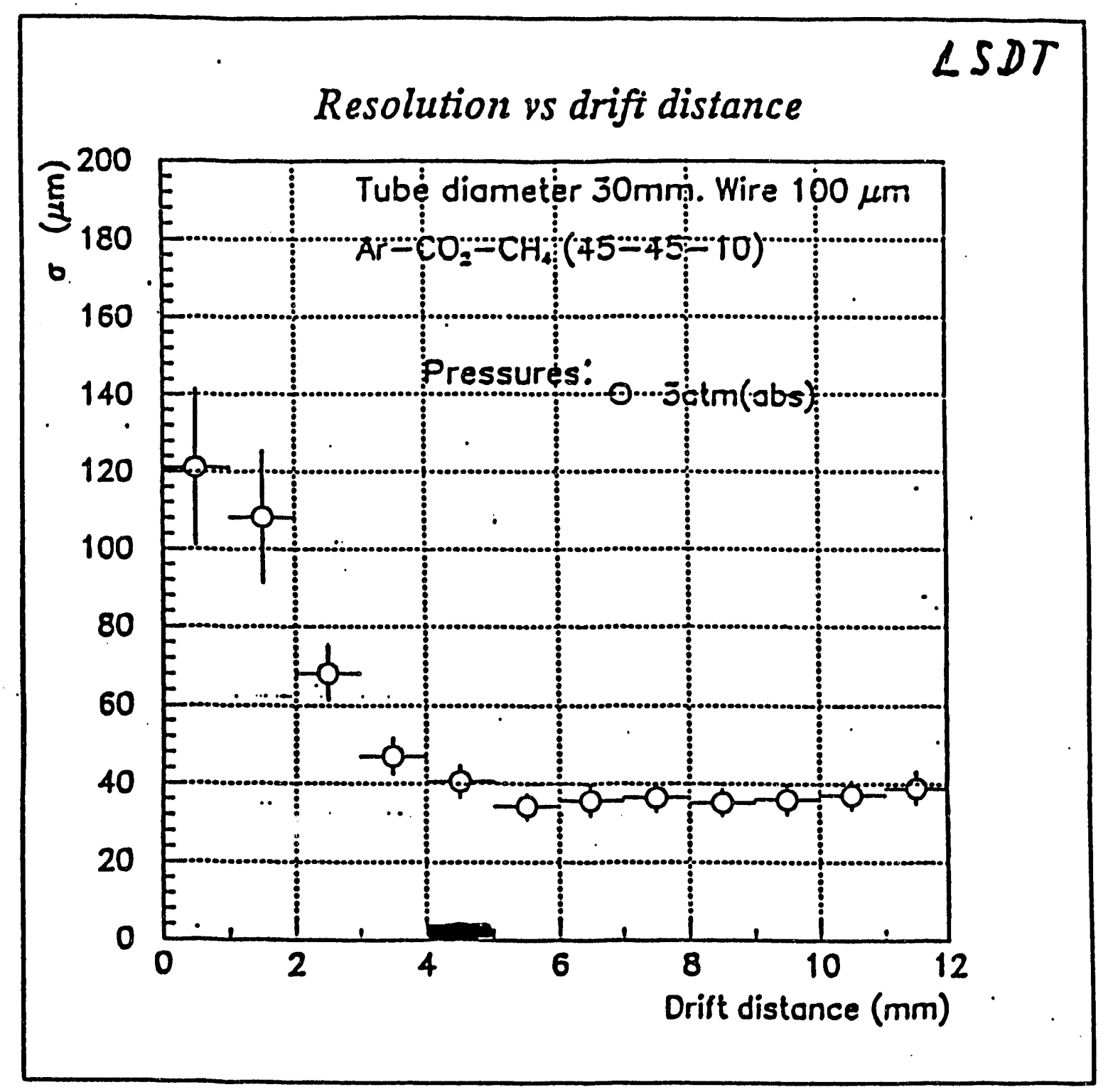

C

Fig. $6.1 .5 d$ 
Drift Chamber Resolution $\left(\sigma_{\mathrm{dIr}}=100 \mu \mathrm{m} /(\mathrm{cm})^{1 / 2} ; \lambda=250 \mu \mathrm{m}\right)$

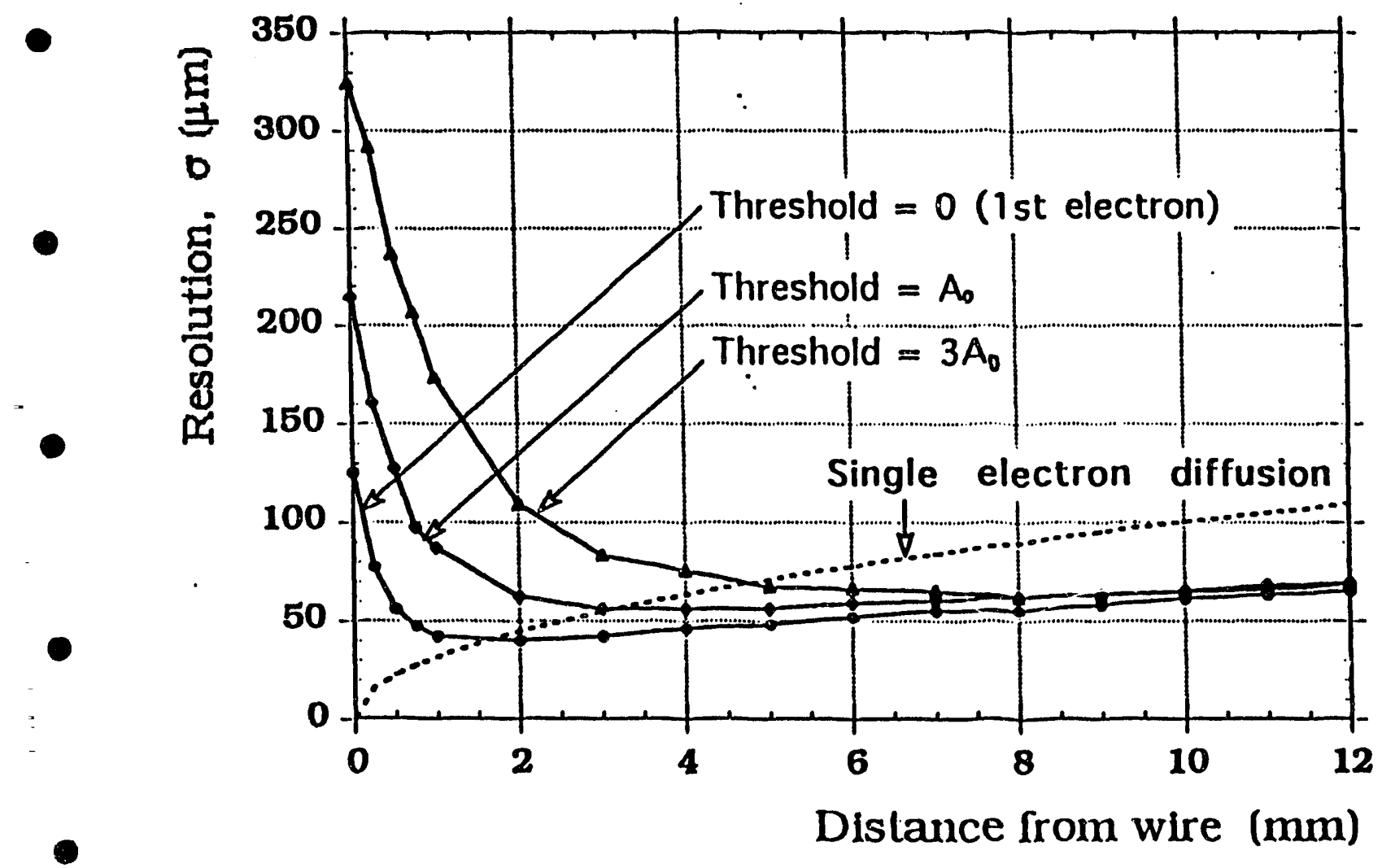

Fig. 6.1 .6 


$$
\mathrm{Ar}-\mathrm{C}_{2} \mathrm{H}_{6}(50 \% 50)
$$

PROP. MODE

\begin{tabular}{|c|c|c|c|c|c|c}
\hline$U_{A} \mathrm{kV}$ & $P_{\text {alm }}$ & $\Delta$ & 2 & 3 & 4 & $1 \ldots 4$ \\
\hline 3.1 & 1 & $(+20)$ & $(-47)$ & $(+0)$ & $(+10)$ & 110 \\
\hline 4.3 & 2 & $(12)$ & $(-52)$ & $(+10)$ & $(+10)$ & 92 \\
\hline 5.9 & 4 & $(+36)$ & $(-50)$ & $(+0)$ & $(+10)$ & 76 \\
\hline
\end{tabular}

$$
\mathrm{Ar}-\mathrm{C}_{4} \mathrm{H}_{10}(25 / 75)
$$

$$
\mathrm{P}=1 \mathrm{~atm}
$$

\begin{tabular}{|c|c|c|c|c|c|c|}
\hline$U_{A}$ kV & \multirow{M}{*}{ Layer } & 1 & 12 & 3 & 4 & $1 \ldots 1$ \\
\cline { 3 - 7 } & $\Delta$ & $\Delta$ & $\Delta$ & $\Delta$ & $\sigma$ \\
\hline 4.1 & prop. & $(+34)$ & $(-52)$ & $(+12)$ & $(+10)$ & 95 \\
\hline 4.5 & strîmer & $(+34)$ & $(-57)$ & $(+10)$ & $(+12)$ & 82 \\
\hline
\end{tabular}

$\sigma$-space resolution (um)

$\Delta$-systematic shifl (umi)

F1g. 6.1 .7 
SENT BY:DALLAS TX

DUBNA RDT: Ar-C RH $_{4} H_{10}(25 / 75)^{917088790048 ; \#}$ at 1 atm

Lढे $\ell_{2 y 2 r} \sim 85 \mu$

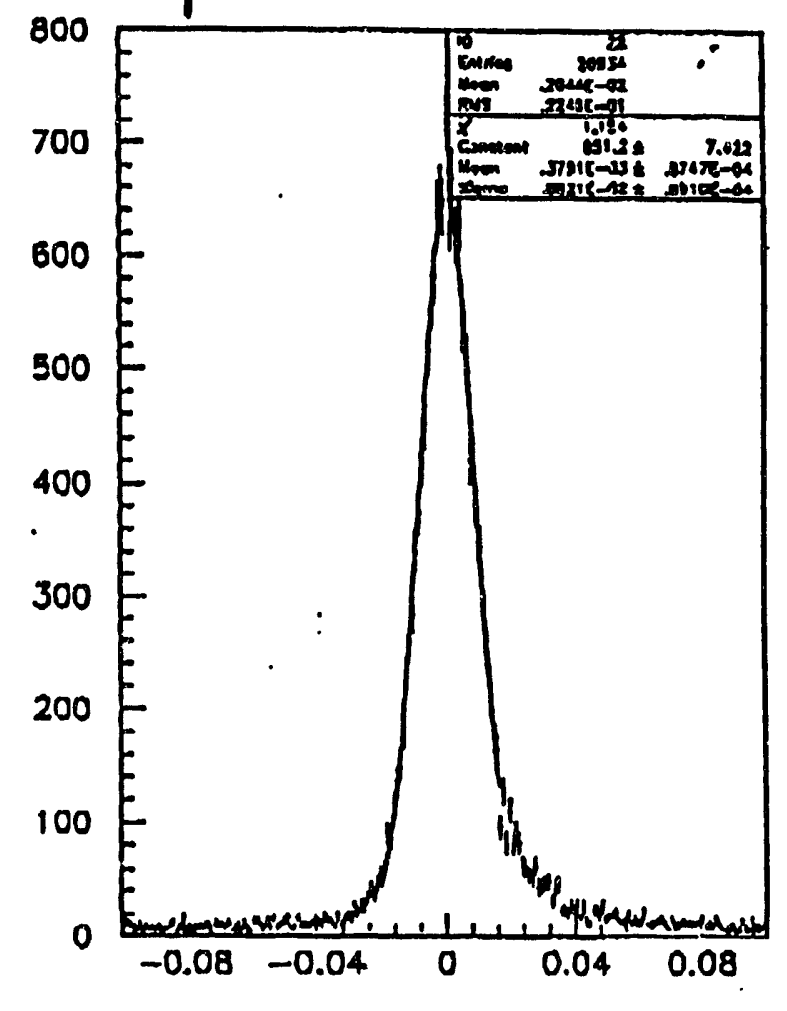

p582
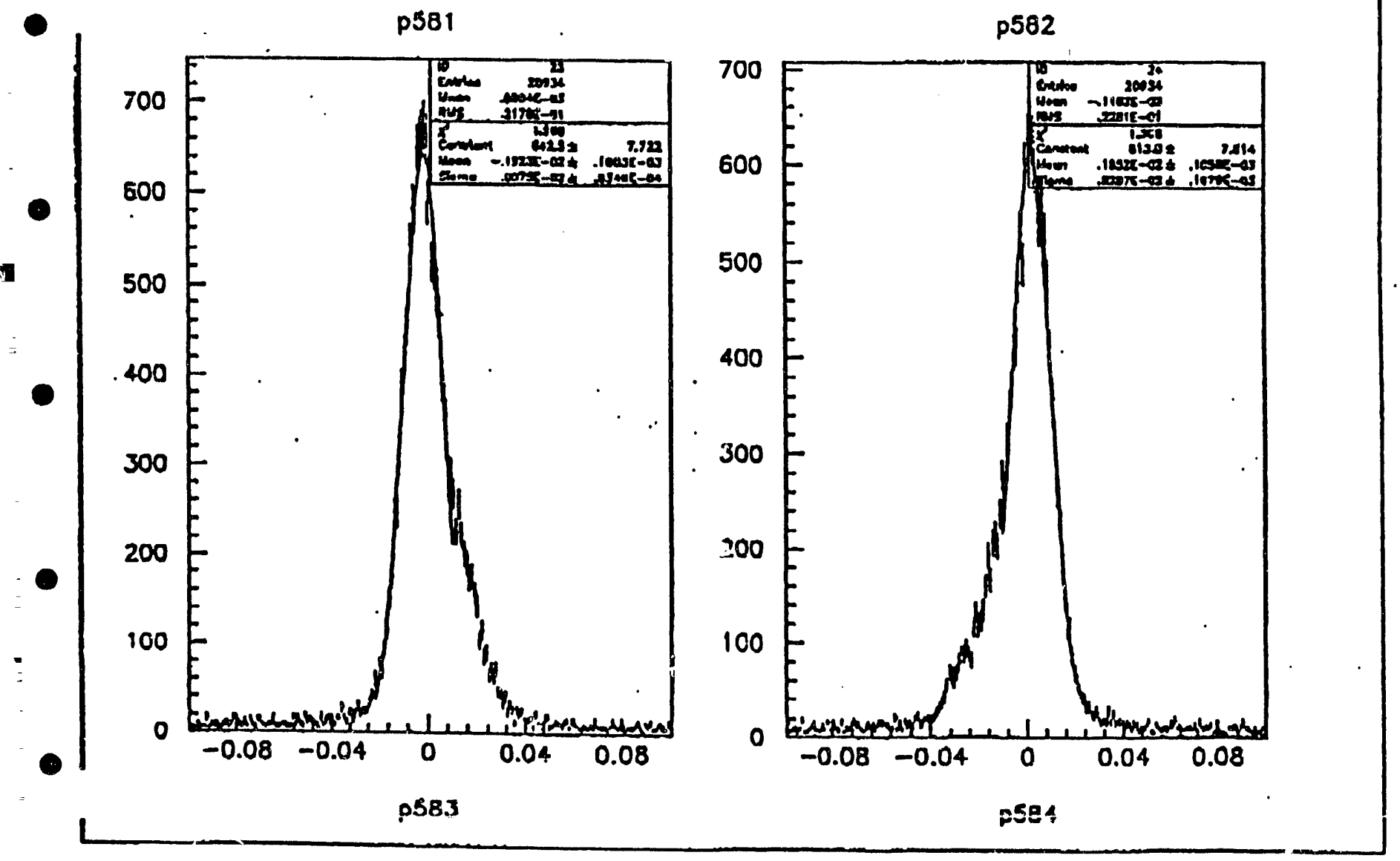

Fig. 6.1.8

- Asymmetrical tails are jue to 


\section{Residuals as a function of tube number}

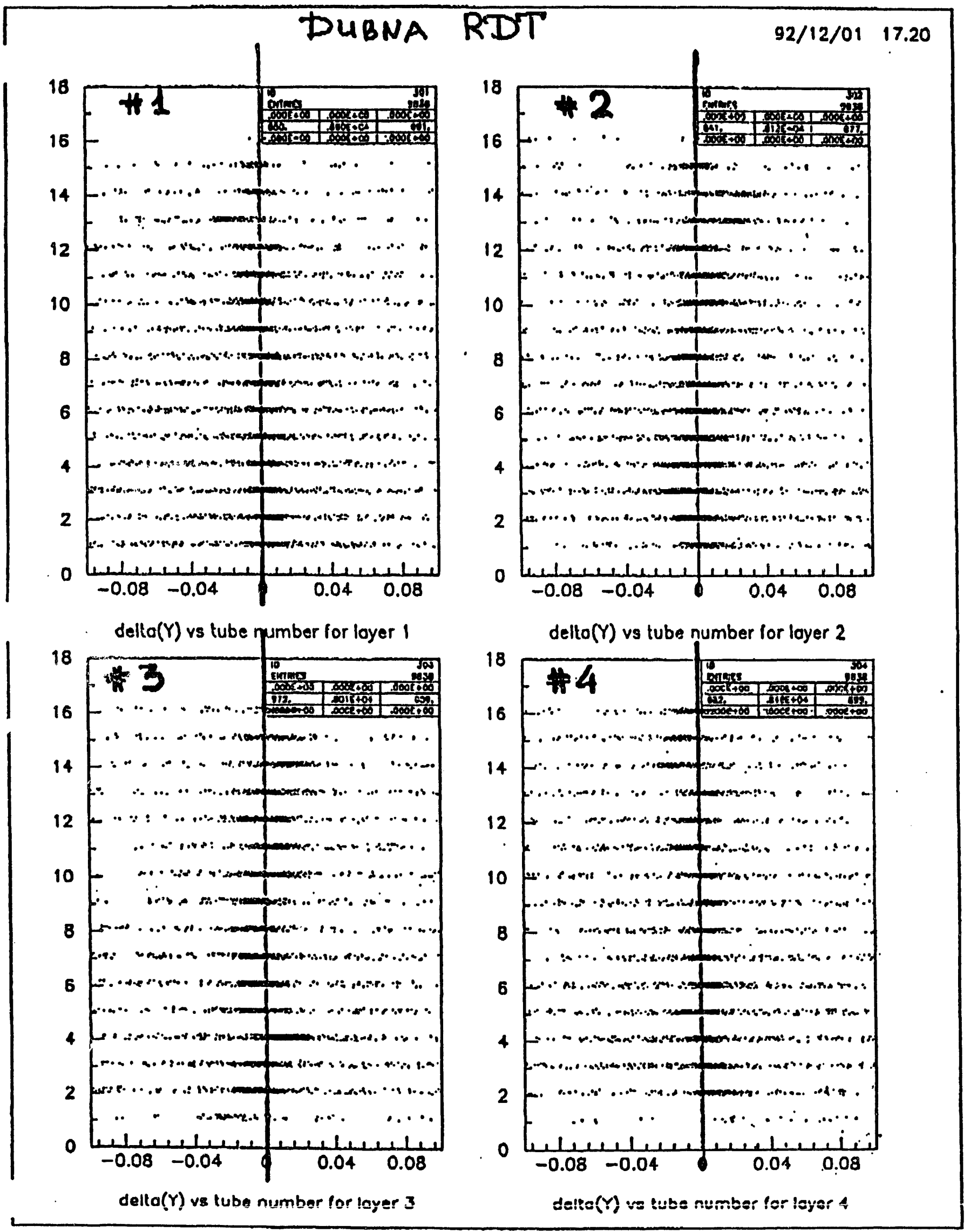




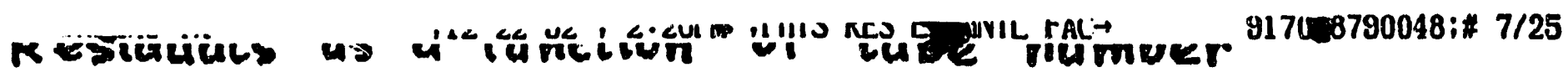
(after parallel shifts of wires)
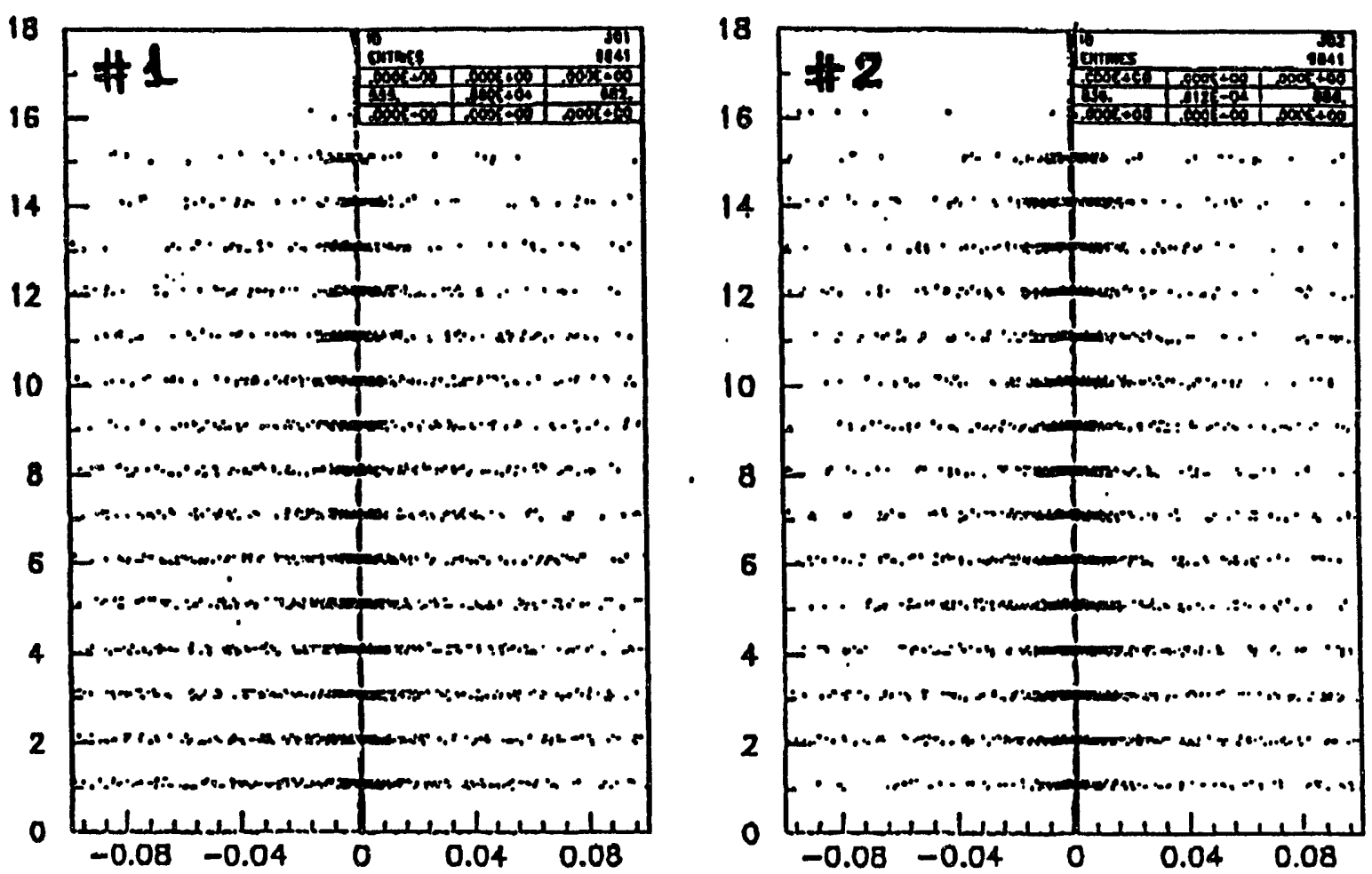

delta $(Y)$ ve tubo number for loyer 1
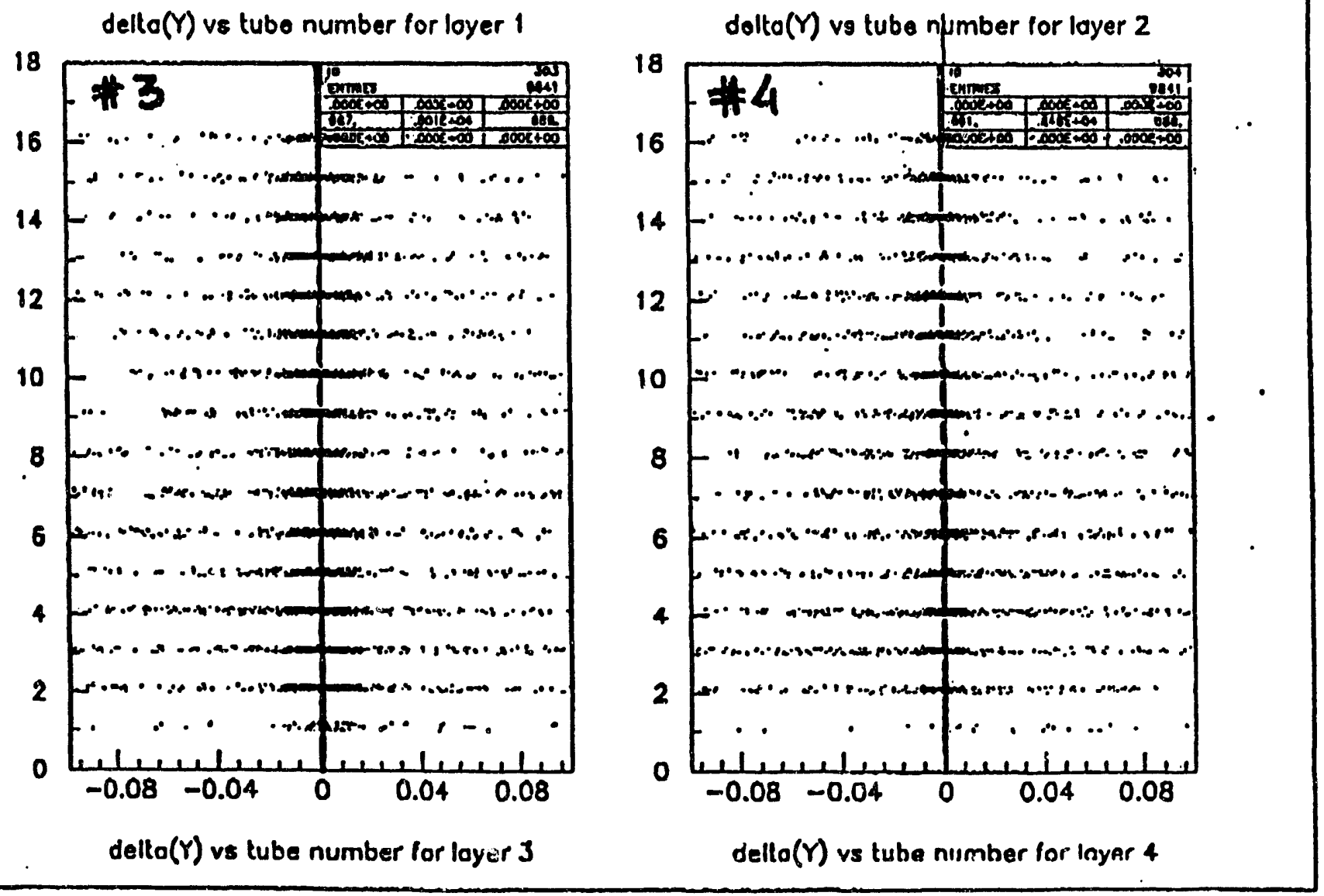

Fig. $6.1 .9 \mathrm{~b}$ 
MU I LI DALLAS IX

$: 12-22-92 ; 2: 27 P M$;PHYS RES EXPMNTL FAC $\rightarrow$

917088790018; $8 / 25$

Rotation of layers

$\uparrow_{\Delta y, \mathrm{~cm}}$ Profile histo's along the wire 92/12/01 08.28
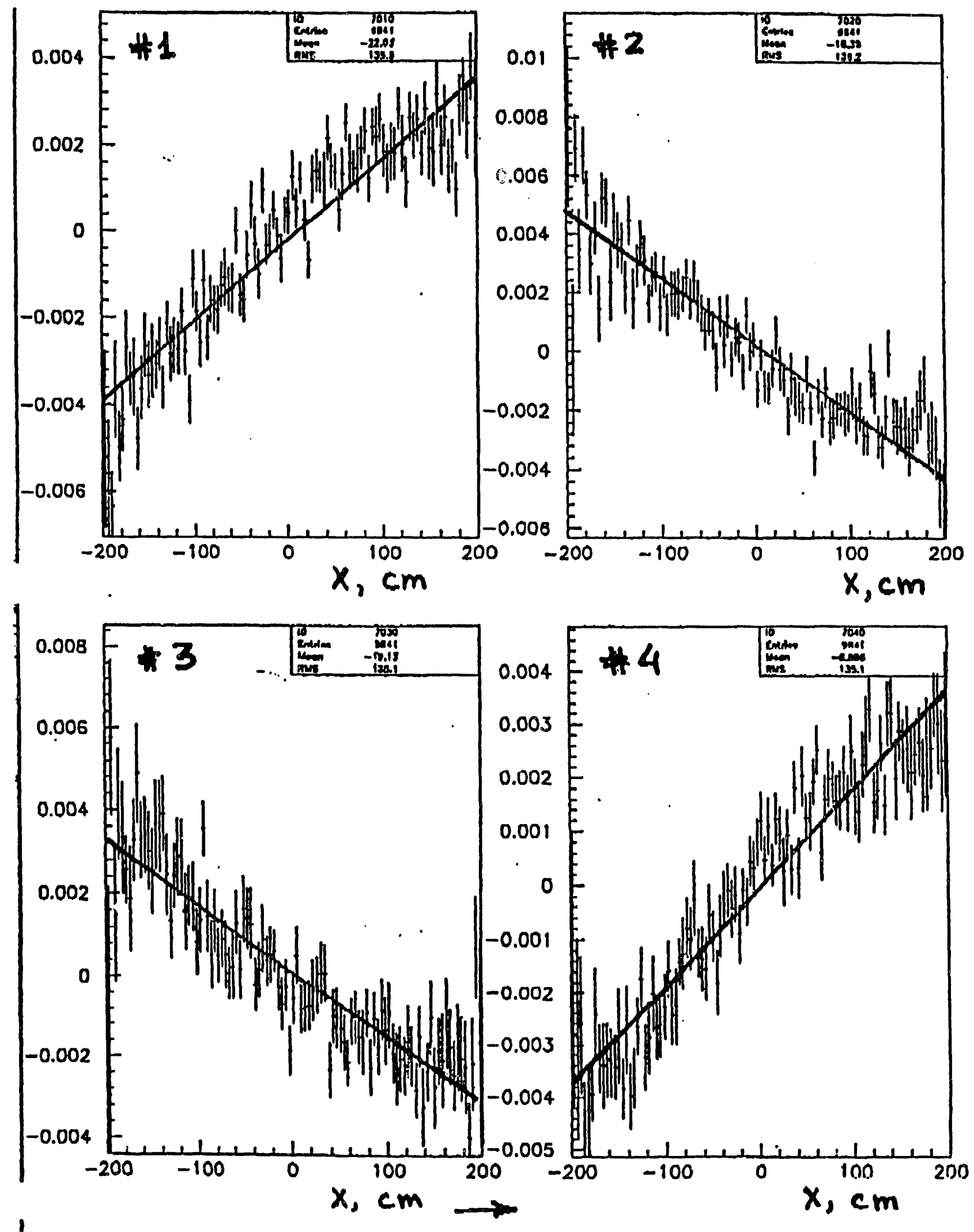

C

i

Fig. 6.1.10

88 


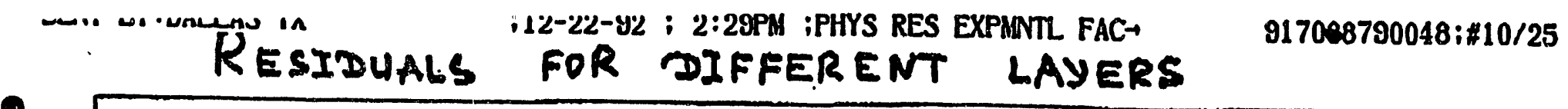

- \#1. 92/12/07 23.27
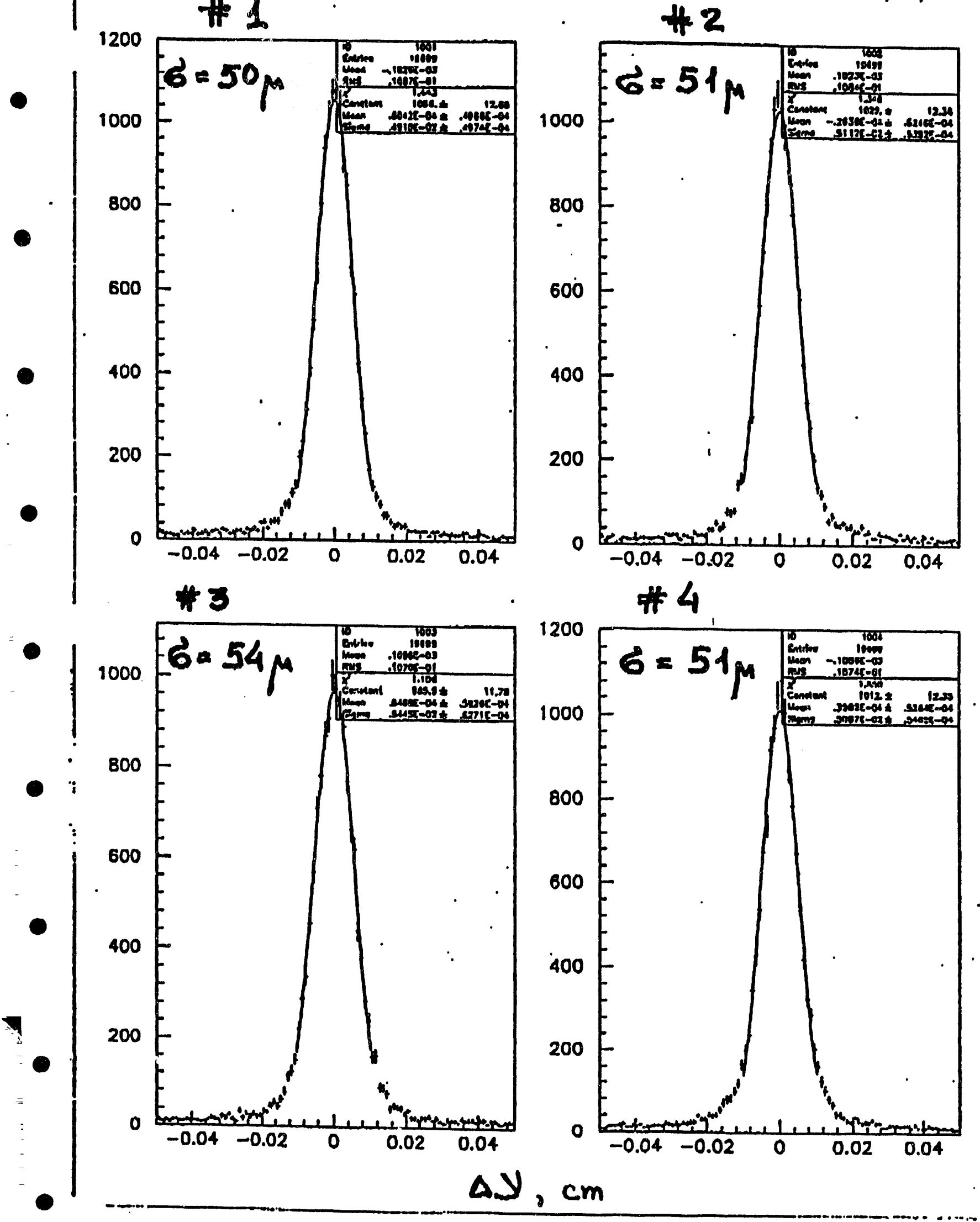

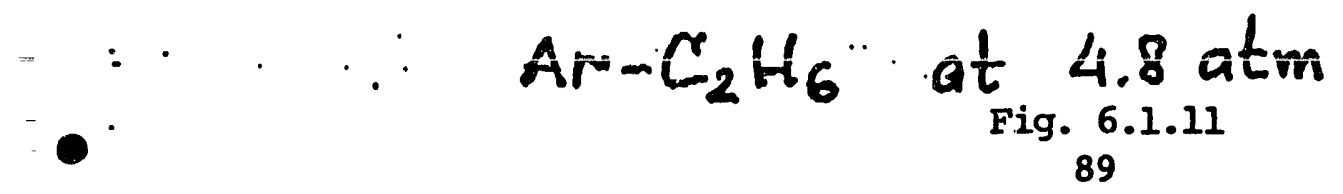


DUBNA RDT's

$\mathrm{Ar}+\mathrm{C}_{2} \mathrm{H}_{6}=50+50$

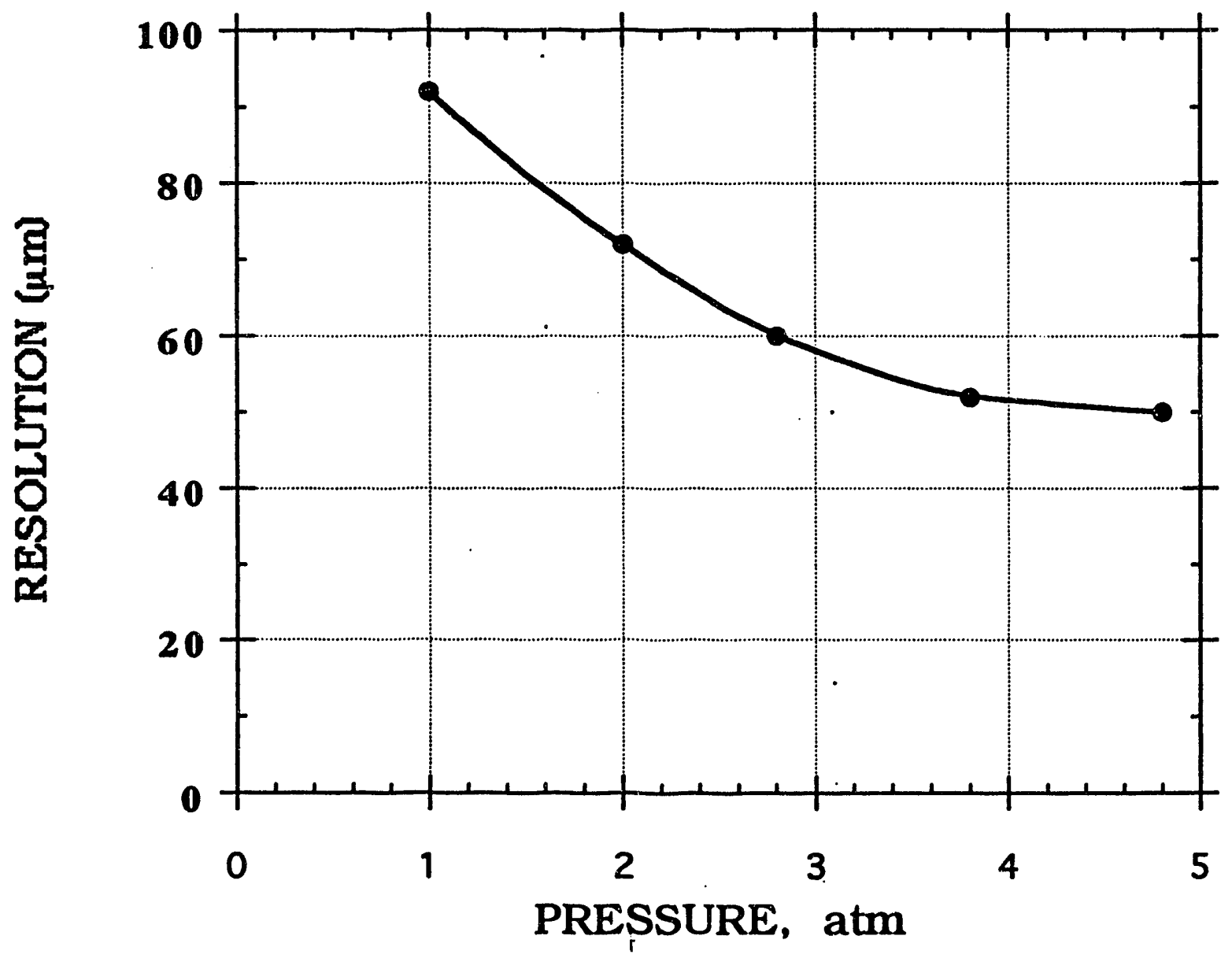

C

C

Fig. 6.1 .12 



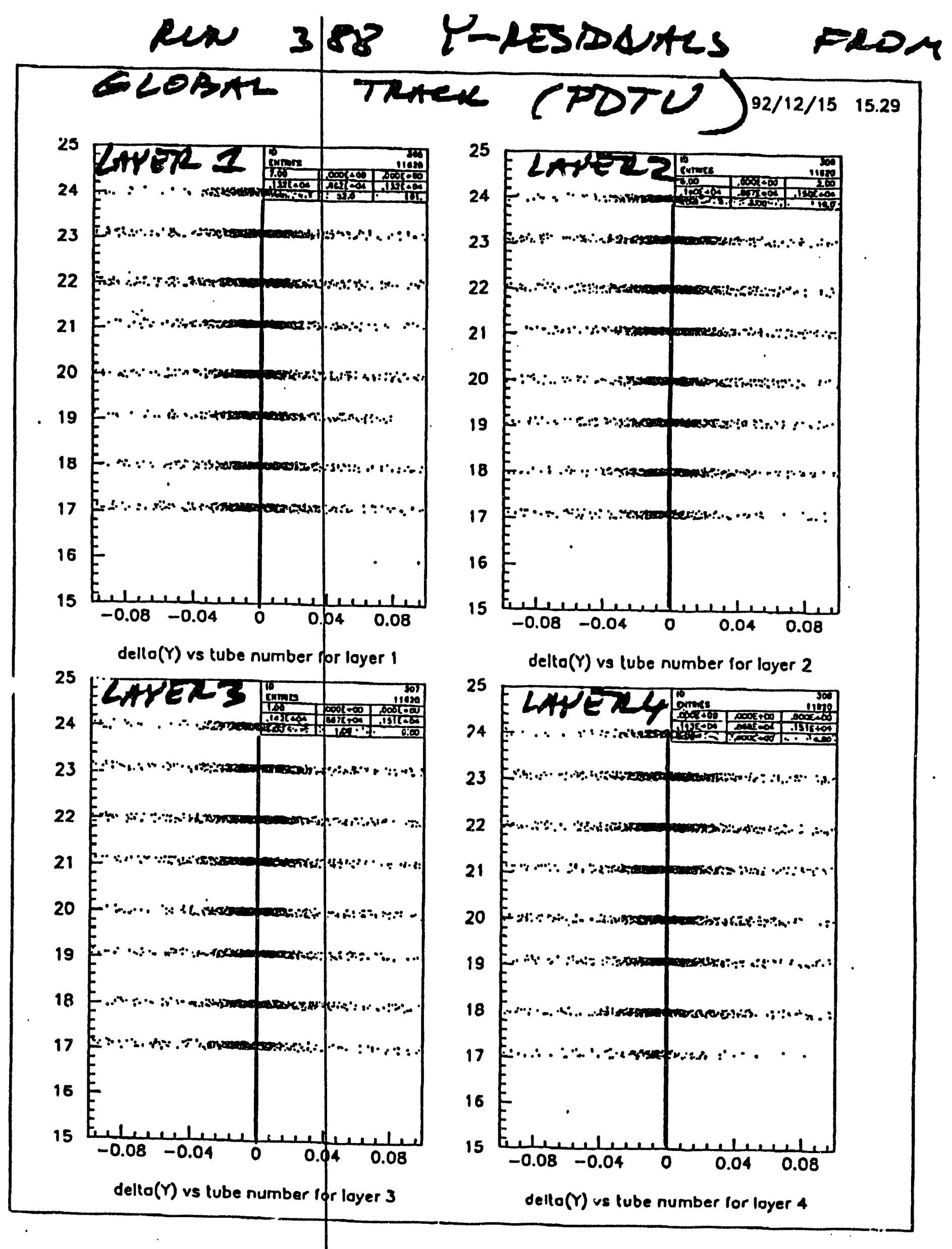

Fig. 6.1 .14 


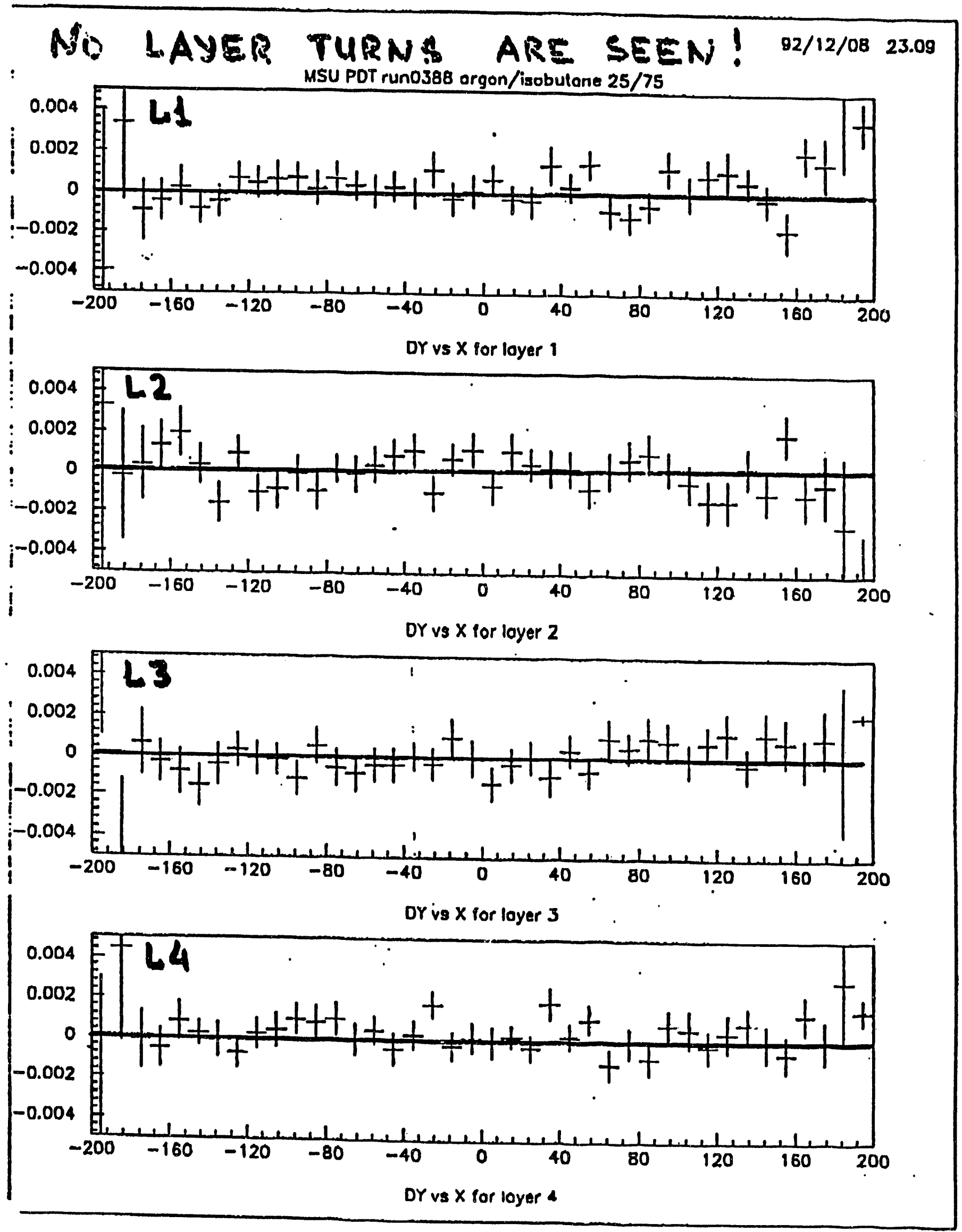

Fig. 6.1.15 


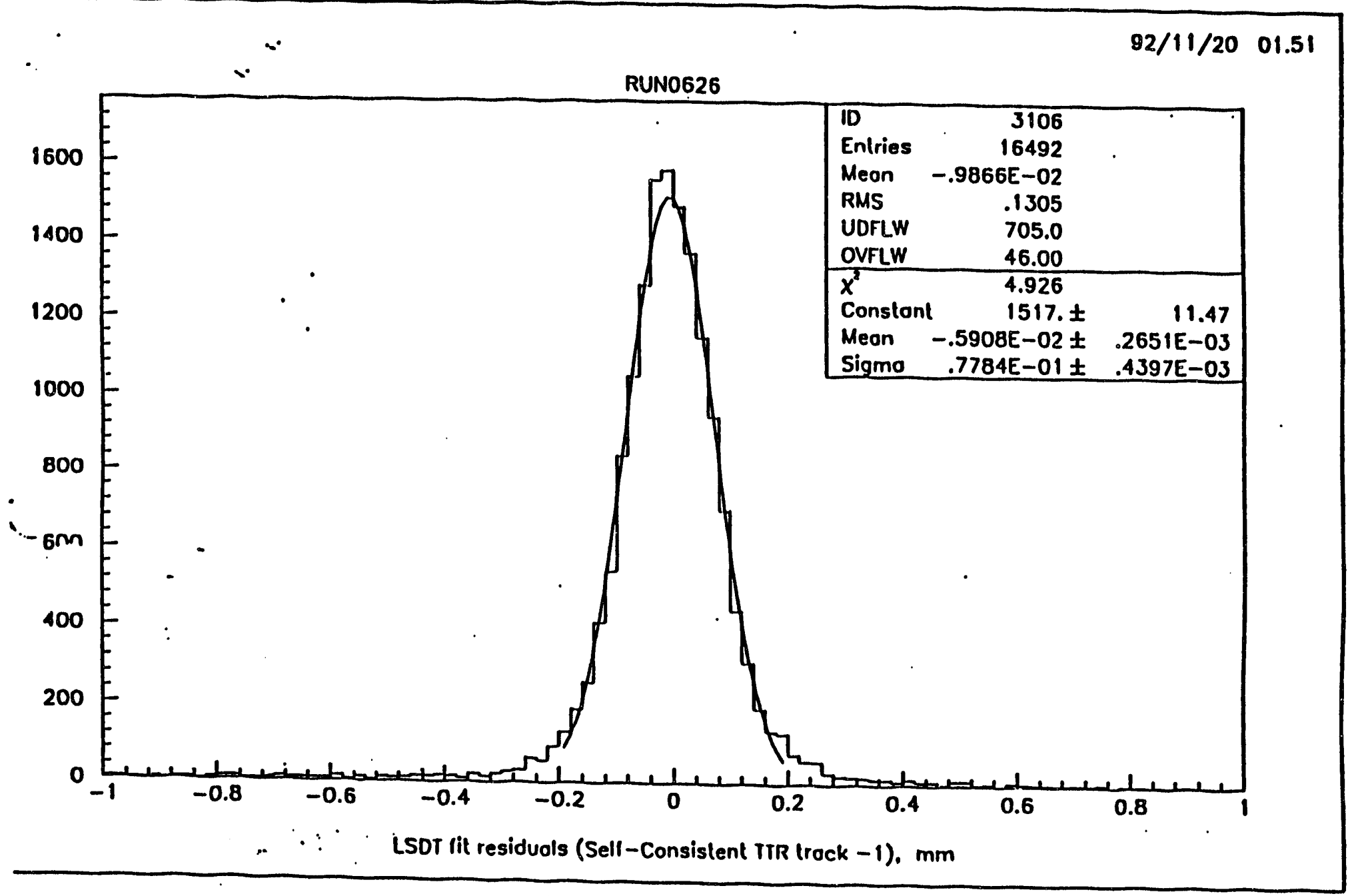

Fig. 6.1 .16

c 


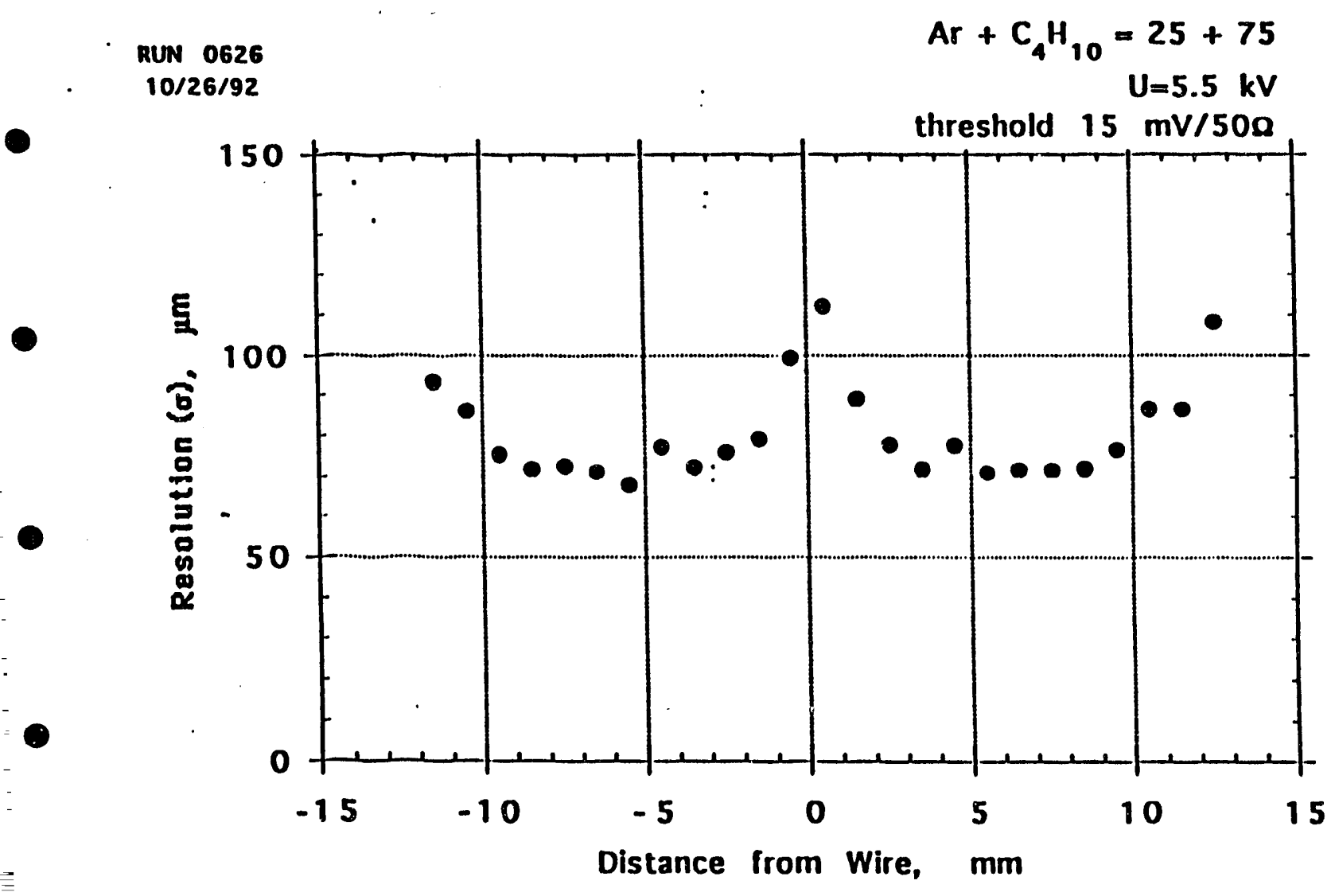

Fig. 6.1 .17 


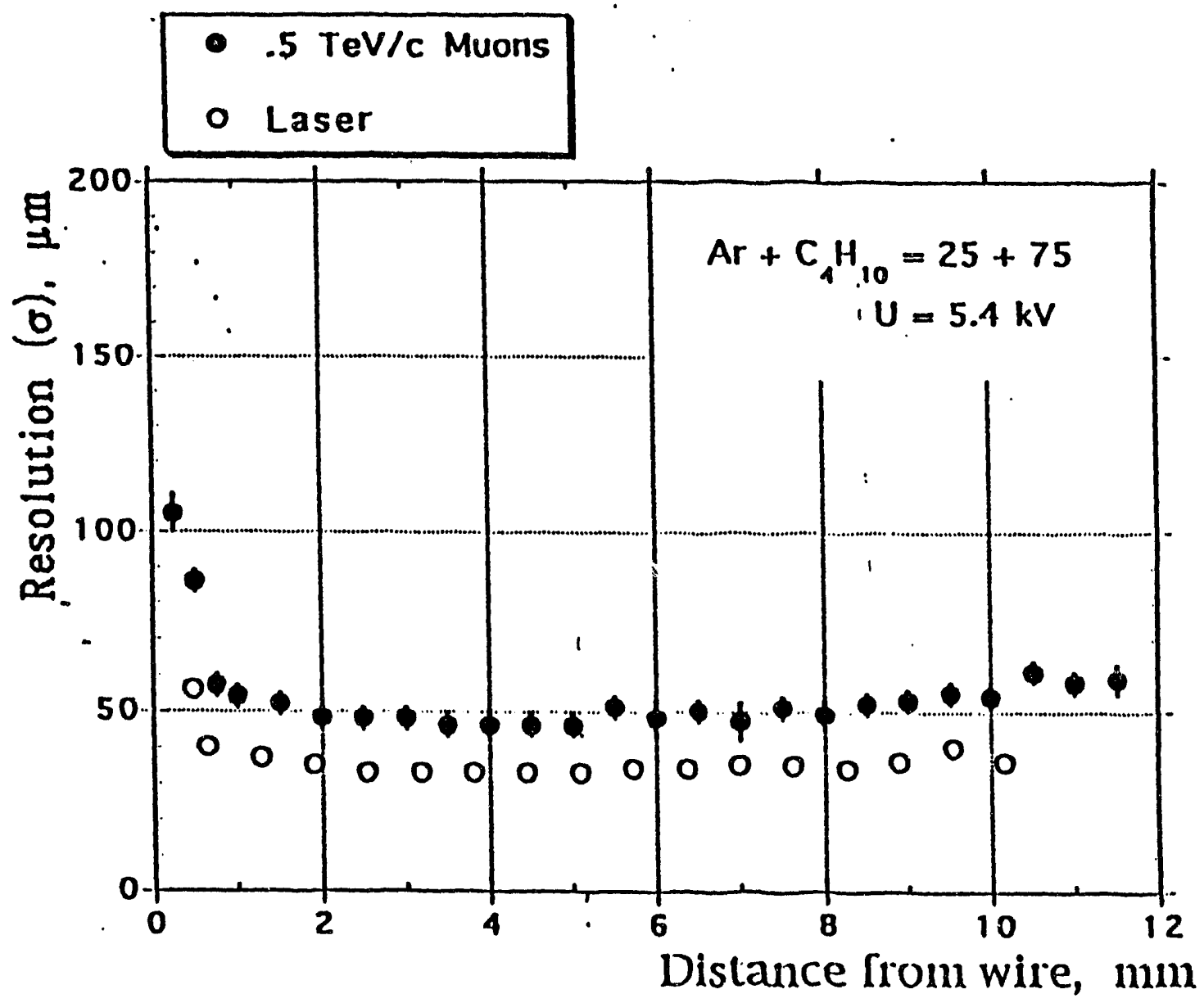

Fig. 6.1 .18 


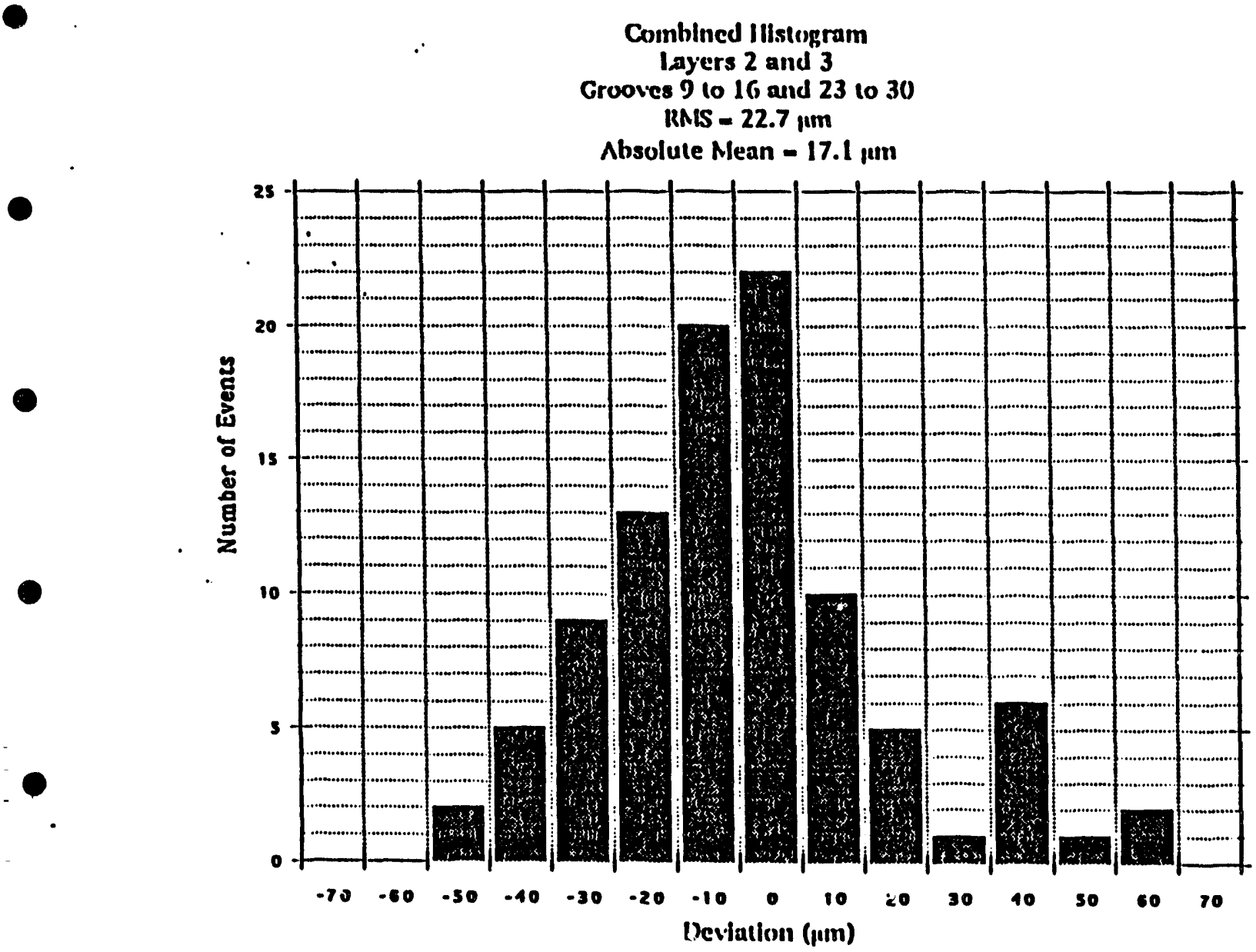

Fig. 6.1 .19 


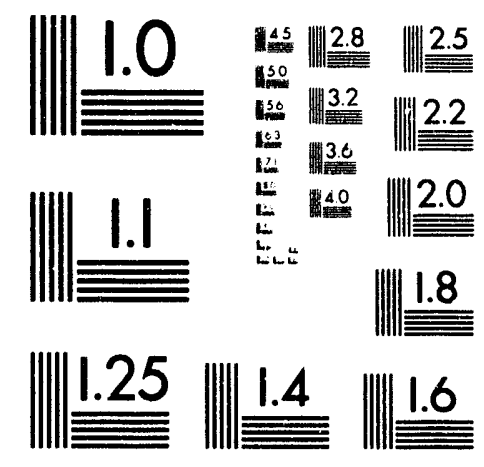



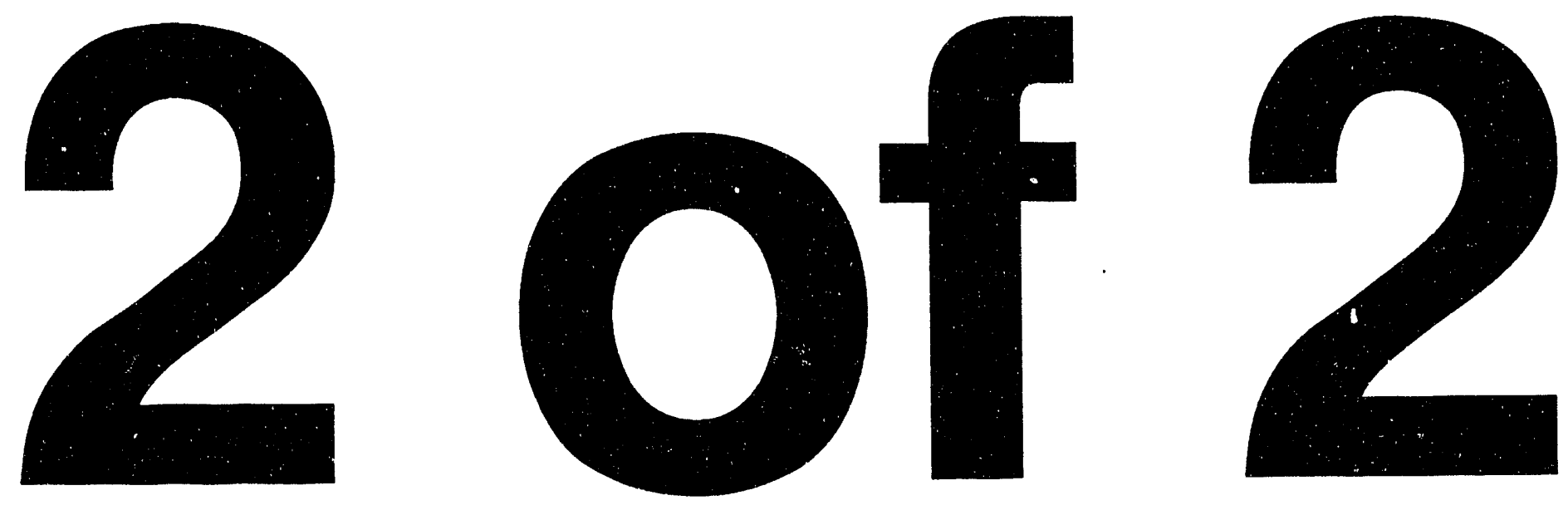


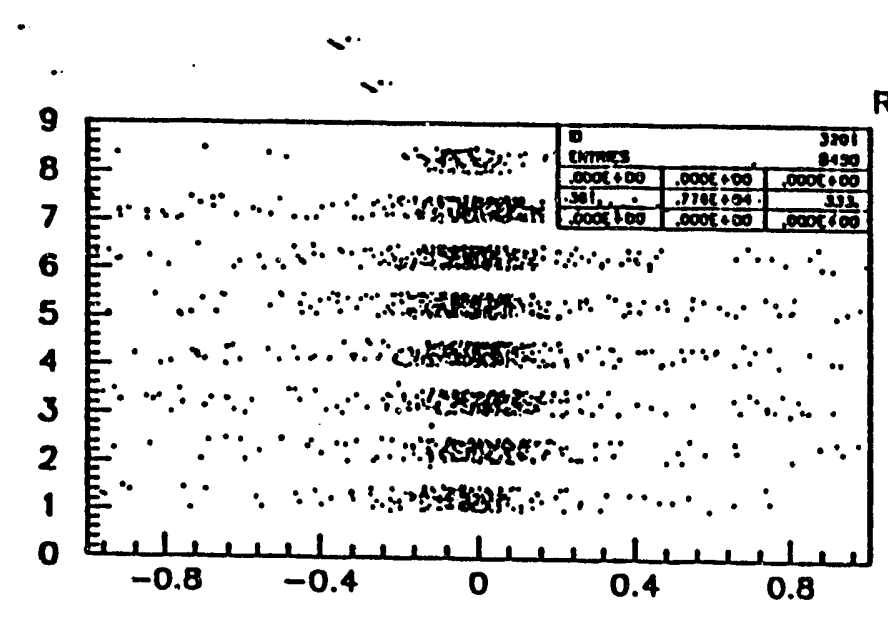

Plone No 1

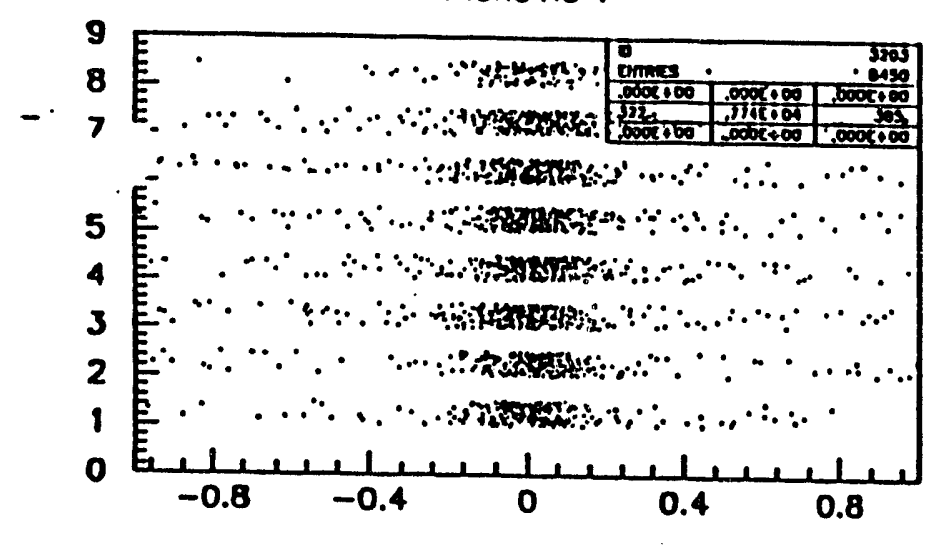

Plone No 3

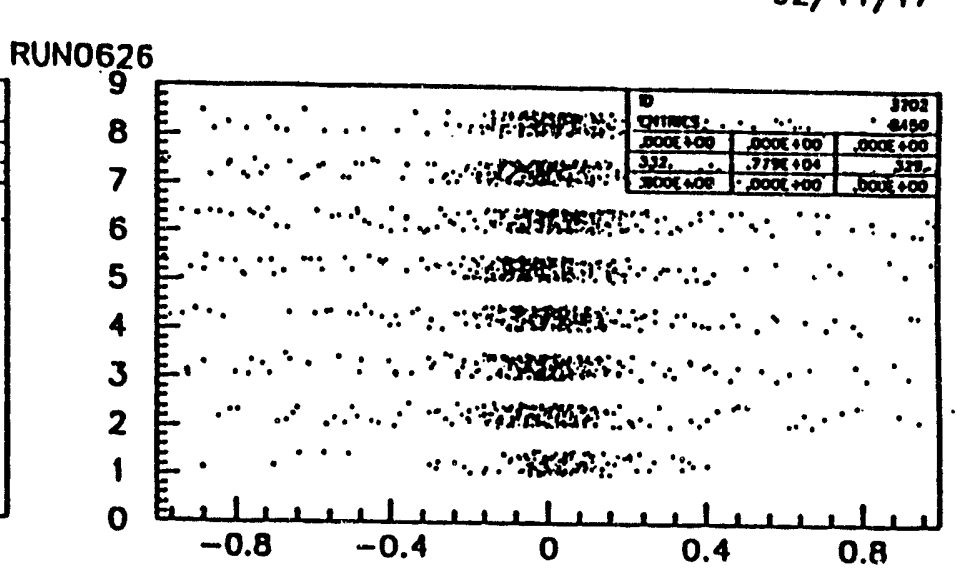

Plone No 2

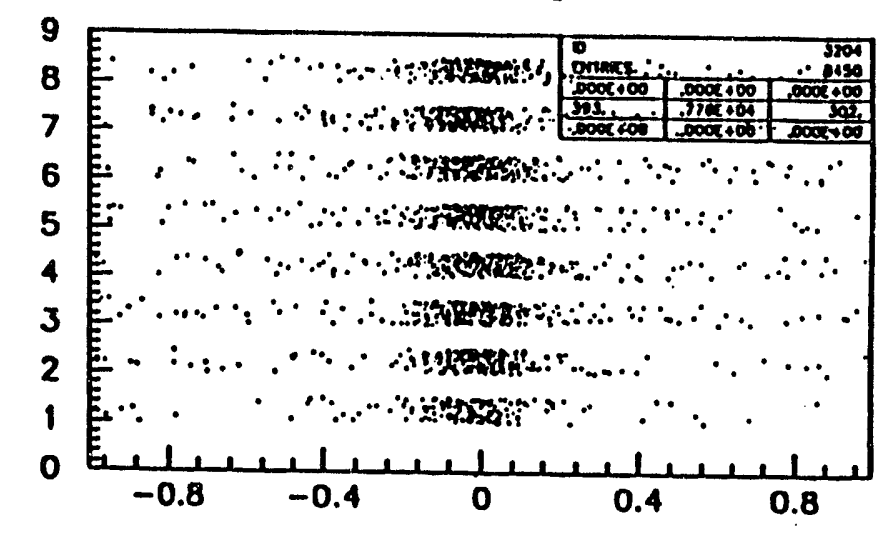

Plane No 4

Fig. 6.1.20a 


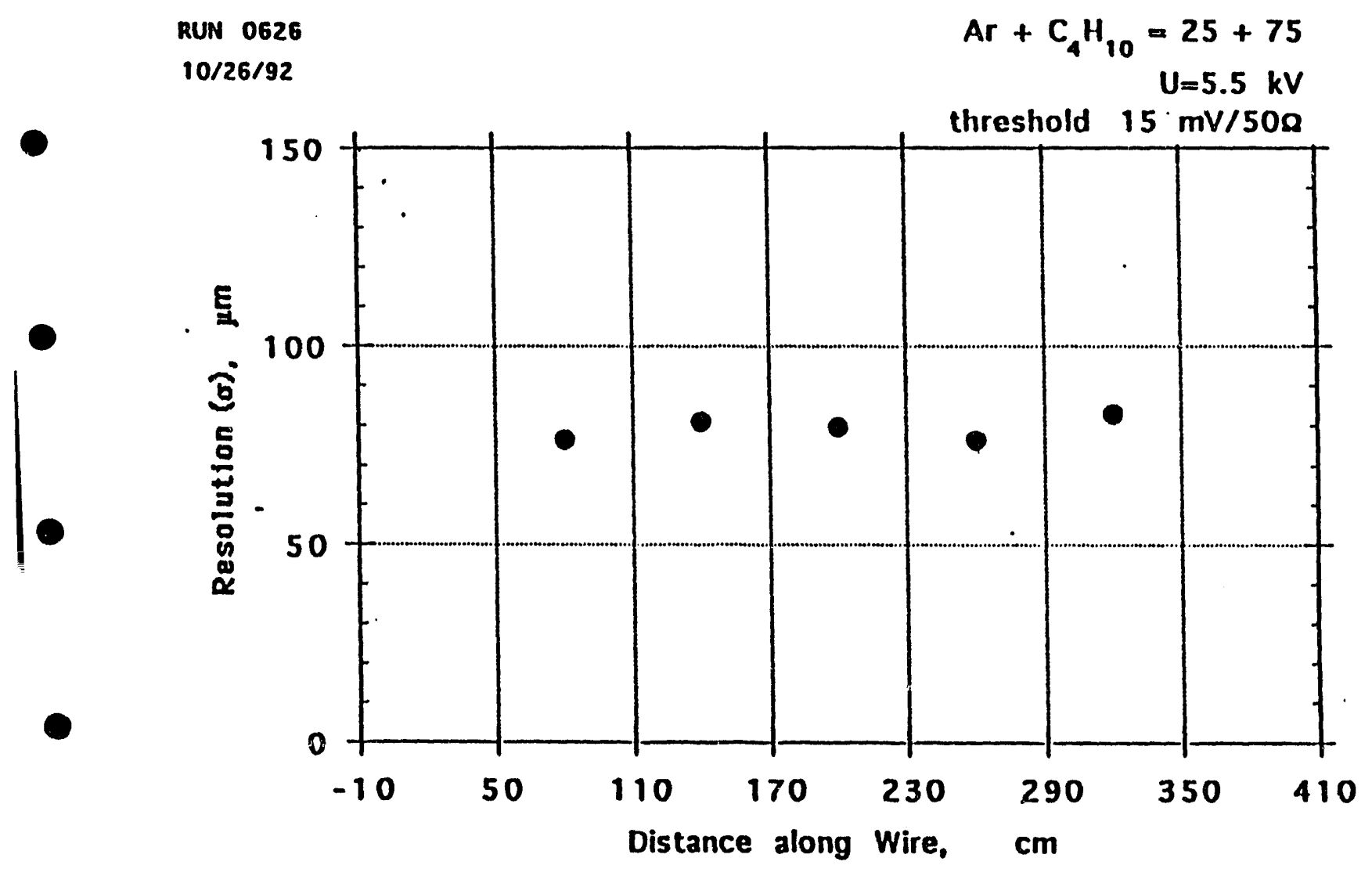

Fig. $6.1 .20 \mathrm{~b}$ 


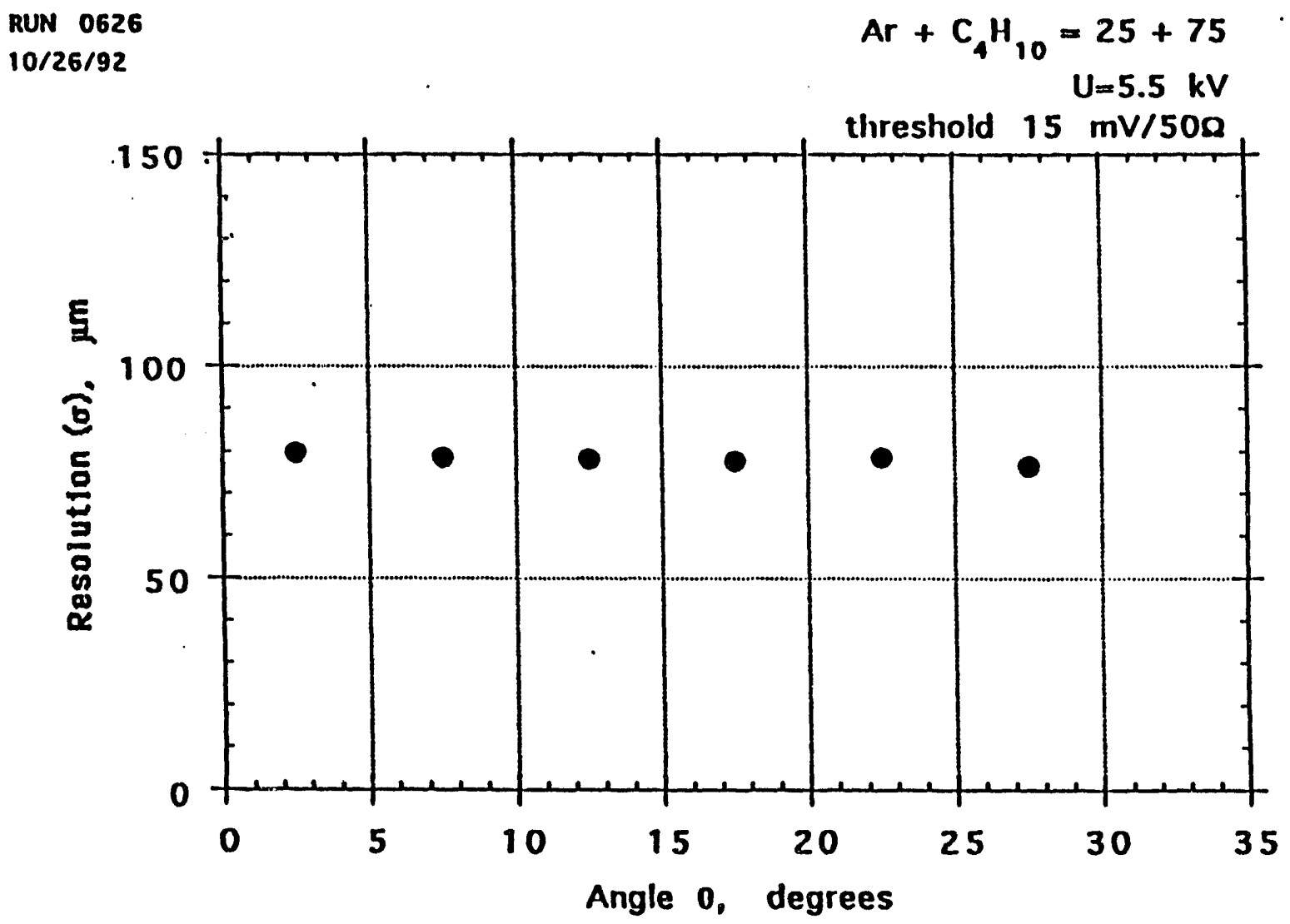

Fig. 6.1.20c 

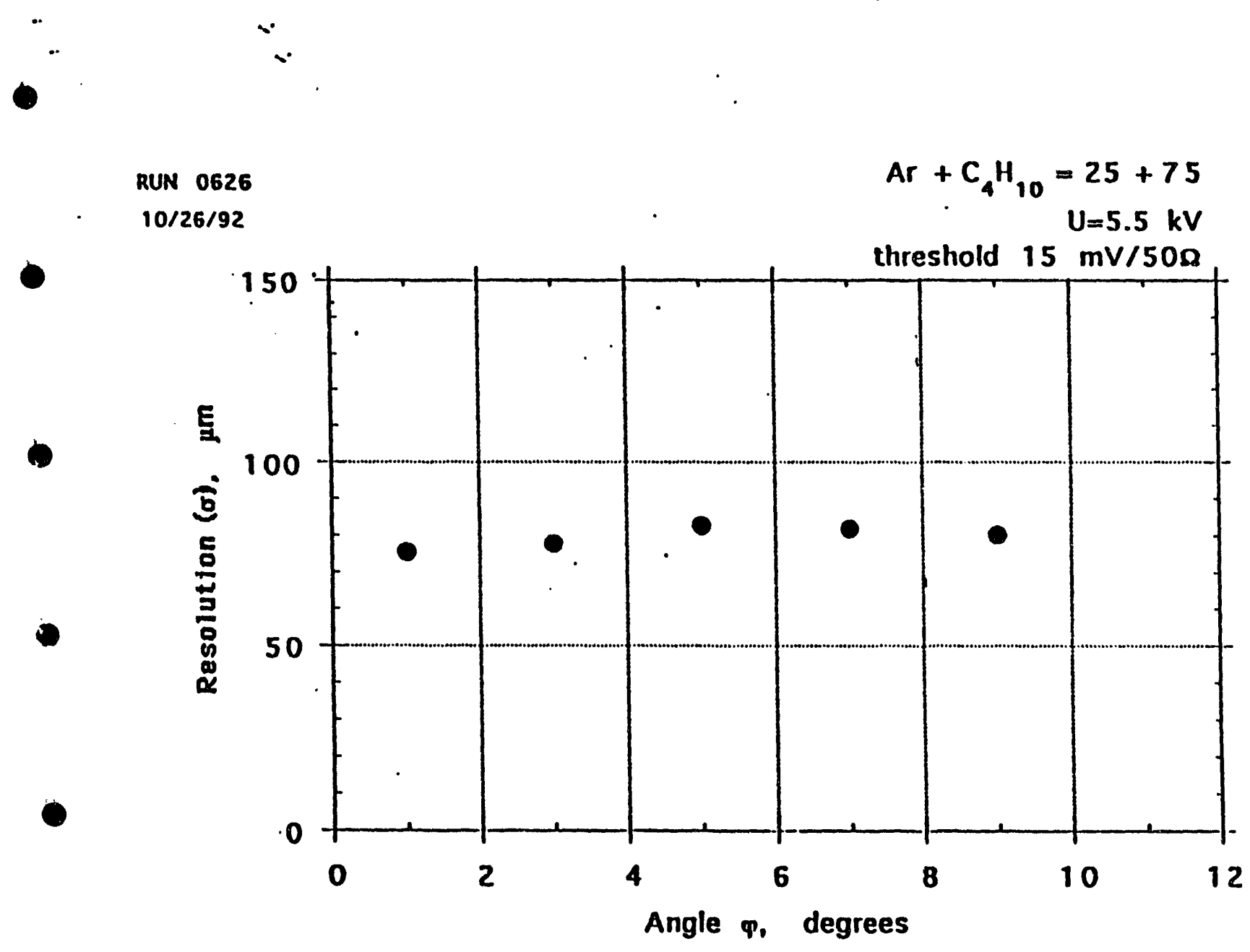

Fig. 6.1.20d 


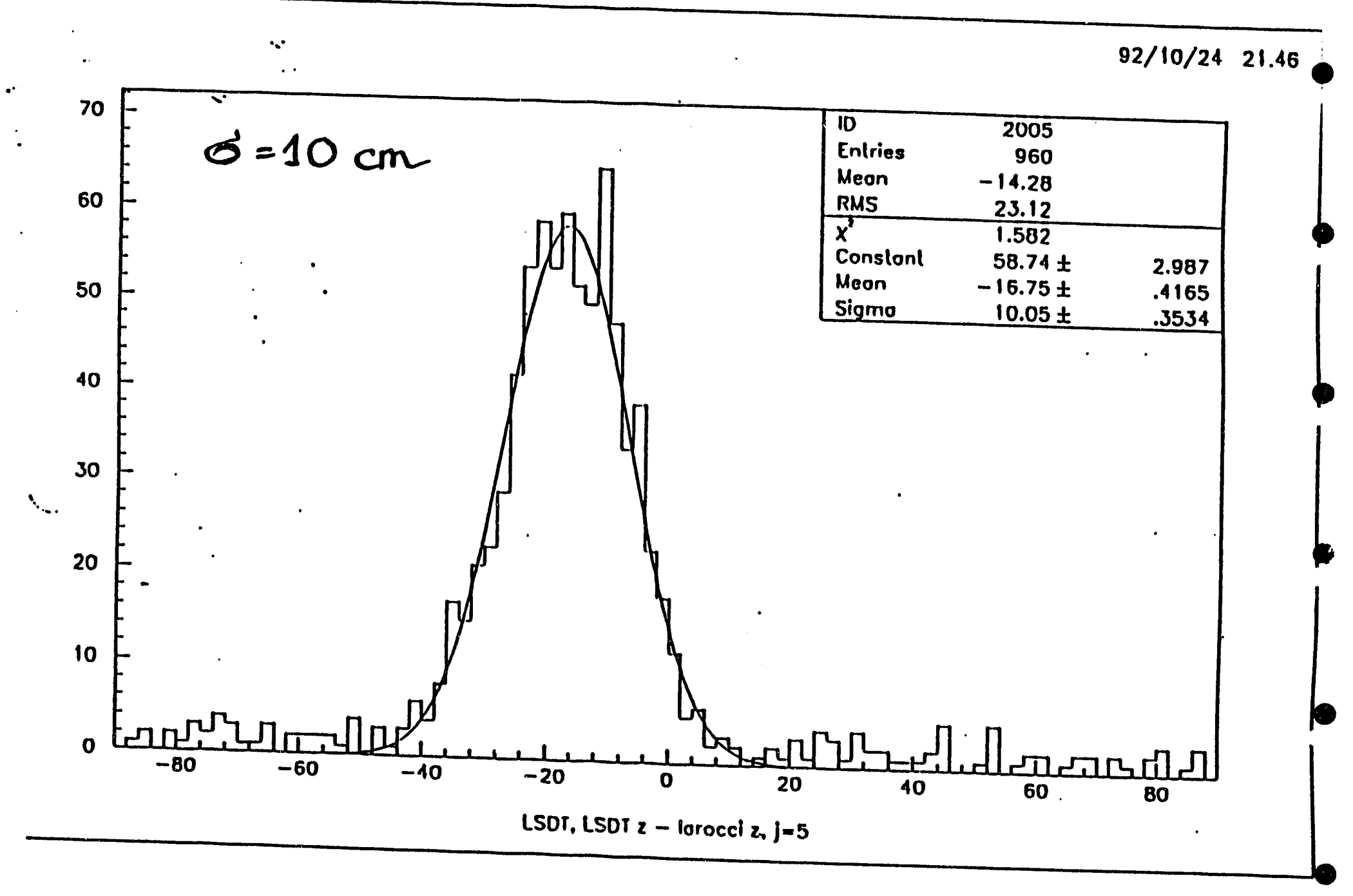

Fị. 6.1.21 


\section{(6.1.1) Correctiuns to Precision Drift Measurements}

\section{(6.1.1.1) Lorentz Angle}

Drift tubes determine the trajectory space point by measuring the drift time which is converted to a distance from the wire by knowing the drift velocity. The time ( $t)$-tospace $(\boldsymbol{r})$ function can be numerically evaluated as:

$$
t=\int_{a}^{r} d r^{\prime} / V_{r}\left(r^{\prime}\right),
$$

where $V_{r}\left(r^{\prime}\right)$ is the radial component (i.e. parallel to the tube) of the mean drift velocity for electrons drifting in the gas under the combined influence of the electric and magnetic fields. If one assumes the average collision time between the electrons and the gas molecules is independent of the magnetic field, the drift velocity can be expressed analytically as a function of the magnetic field by the following:

$$
V_{r}(r)=V_{0}\left(1+\left(\omega_{\tau} \tau\right)^{2}\right) /\left(1+(\omega \tau)^{2}\right),
$$

where $V_{O}$ is the drift velocity without magnetic filed, a quantity dependent on the gas properties and the electric field, and $\omega=|\omega|$ which depends on the gyration angular frequency for the electron circling in the magnetic field. The vector quantity $\omega=\left(\omega_{\mathrm{r}}, \omega \mathrm{T}, \omega_{\mathrm{Z}}\right)=(\mathrm{e} / \mathrm{m}) \mathrm{B}$ denotes the gyration components: $\mathrm{r}$ in the radial direction of the wire, $Z$ parallel to the wire, ant $T$ is perpendicular to $r$ and $Z$. The Lorentz angle $\theta$ is related to the magnetic field by the following relation:

$$
\tan \theta=\omega \tau \text {. }
$$

By measuring:

- the drift velocity $V_{O}$ as a function of electric field for $B=0$

- the Lorentz angle to obtain $\omega \tau$

we can obtain the space-time function needed to correct for the Lorentz angle using eq. (1) above. Since the correction is small we need to know the B-field to only about $10 \%$.

(a) Application in the Barrel Region:

The isochrons of the time-to-distance relation in the case of the B-field axial to the wire are circles concentric with the wire for the barrel case, or for the radial field component in the endcap. Hence the Lorentz angle correction in this case is independent of the angle of the muon incident to the plane of the muon chambers and is therefore especially simple. To study the influence of the magnetic field on $V_{r}$ 
in this case, a sample tube was operated in a $0.8 \mathrm{~T}$ magnetic field at MIT. The result is that for a typical gas (3:1 - argon-isobutane) the drift time is increased by about $8 \mathrm{~ns}$ out of about $250 \mathrm{~ns}$ for $\mathrm{r}=10 \mathrm{~mm}$ from the wire at $\mathrm{B}=0.8 \mathrm{~T}$. This small change is easy to correct and has been shown not to degrade the resolution by more than $10 \%$.

\section{(b) Application in the EndCaps:}

In this case the $\omega_{\mathbf{r}}$ term is not zero except in the case of normal incidence to the chamber plane. Letting $\xi$ be the angle of incidence of the muon to an endcap chamber plane, we find that $\xi<6.5^{\circ}$ and the term $\omega_{\mathrm{r}}=(\mathrm{e} / \mathrm{m}) \mathrm{B}_{\mathrm{Z}} \sin (\xi)<25 \%$ of the total Lorentz angle correction. In the off-line analysis the angle $\xi$ will be reconstructed, and the more complicated electron trajectory calculated.

As a demonstration of this well-known correction, a cosmic ray experiment (PBAR) employed drift tubes in a $0.9 \mathrm{~T}$ non-uniform field ( $30 \%$ variation) to precisely measure momentum. Fig. 6.1.1.1 shows the measured vs. calculated distance - time relation for the B-field parallel to the axis of the tube, and in Fig. 6.1.1.2 for the B-field perpendicular to the tube. Note that in both cases the measured distance-time relation agrees to within a few \% of the calculated one. Hence the resolution should not be significantly degraded by this effect.

\section{(6.1.2) Wire Sag}

The present design of RDTs for the muon system is based on cylindrical Al tubes of various lengths up to about 4 meter long and $2.5 \mathrm{~cm}$ in diameter. The wires would be strung down the tube centers and would have their ends fixed at precision end-plates without using bridges. We have shown that allowing the wires to sag will be acceptable, even for 4 meter RDTs, and the wire position will be calculable to sufficient accuracy based on knowledge of the resonant frequency of the wire and the high voltage. It has also been shown that deviation of concentricity of the wire center from the tube center is at an acceptable level and that electrostatic instability will not be a problem.

Fig. 6.1.2.1 illustrates the basic components of a drift tube, and formulae for wire displacement due to gravity and electrostatic forces are also given. The critical voltage at which the electrostatic instability sets in, V(critical), is also given, which, for our application, is much larger than the voltage required for RDT operation.

Fig. 6.1.2.2 gives formulae for calculating the electrical field inside the drift tube, for the general case of a wire which is offset from the tube center. Using this formulae, it is possible to calculate tolerances acceptable for wire/tube a-concentricity (which contribute to tracking resolution errors due to deviation of the true space-lime function from that assuming the wire and tube wall to be concentric). As examples, Fig. 6.1.2.3 shows the drift time error for the case of an offset of $1 \mathrm{~mm}$, for a $2 \mathrm{~cm}$ 


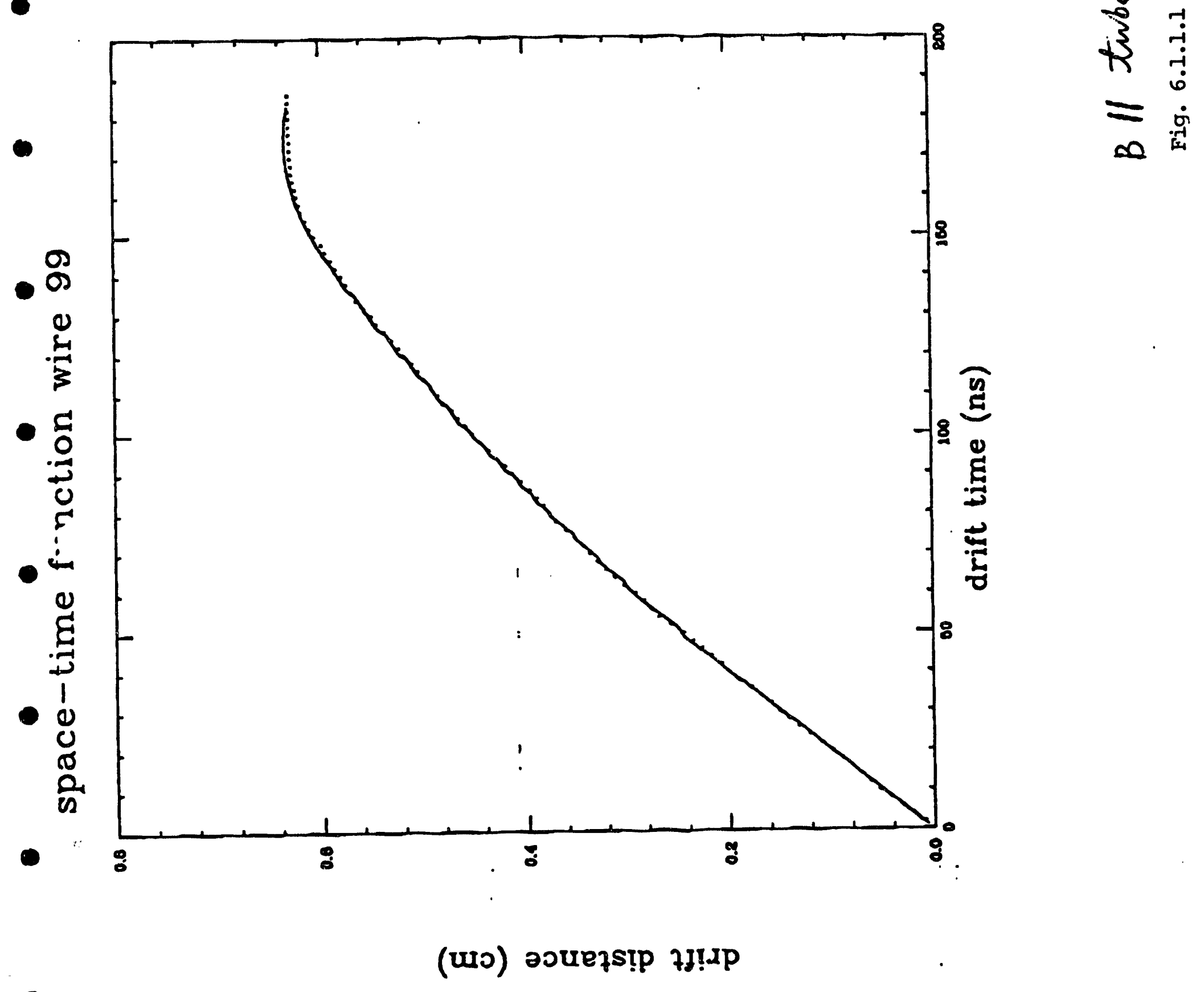




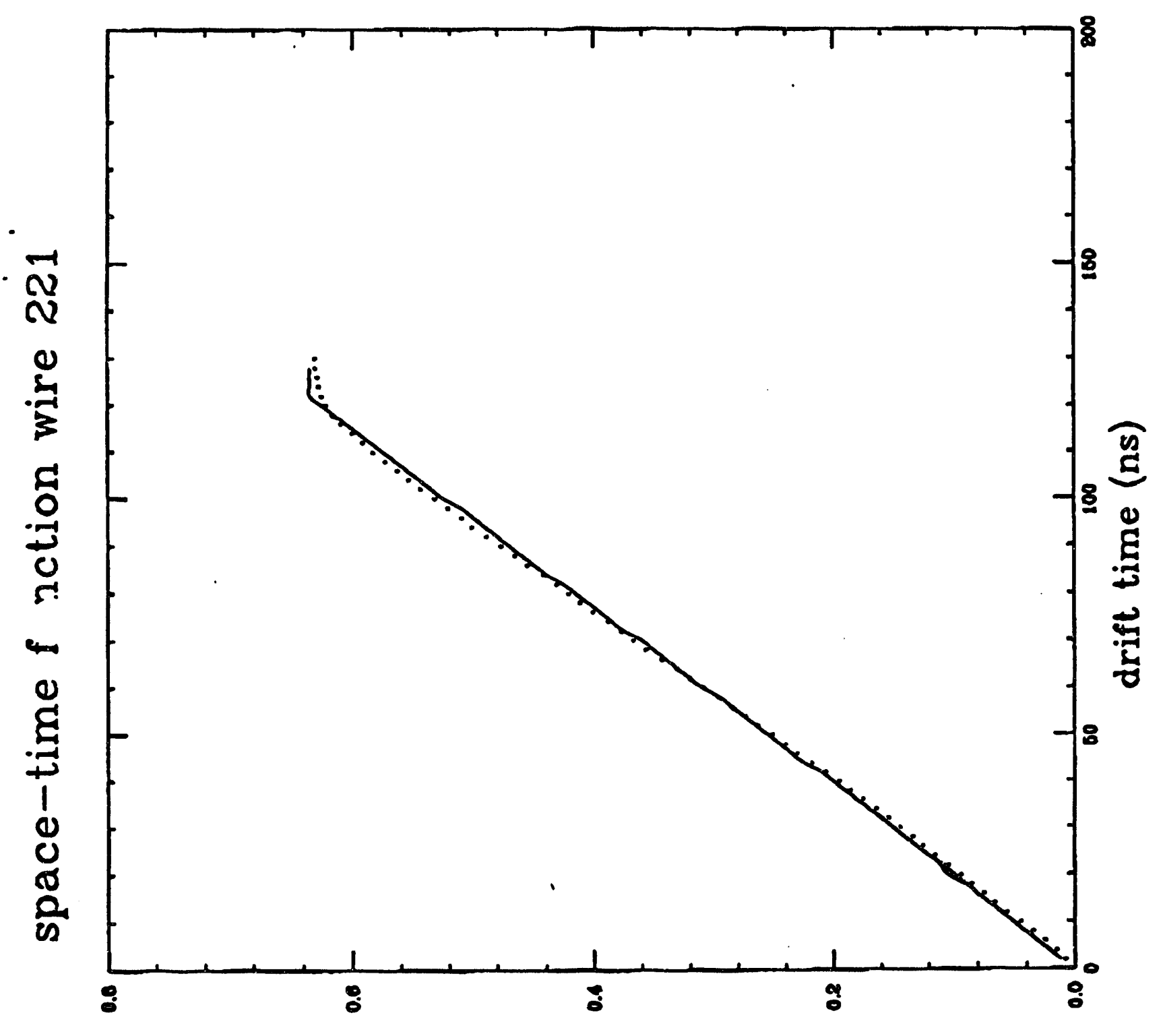

(wo) əoueqsip 7I!גp

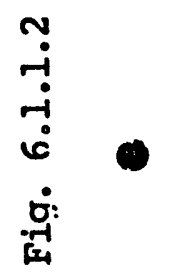

$\frac{4}{3}$

$\infty$

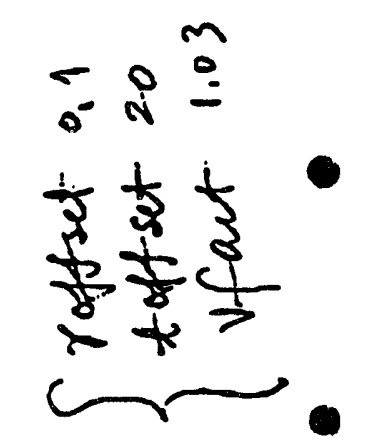


diameter tube and 25 micron wire, using the $\mathrm{HRS}$ gas $\left(89 \% \mathrm{Ar}, 10 \% \mathrm{CO}_{2}, 1 \% \mathrm{CH}_{4}\right)$ at $1 \mathrm{~atm}$ and 2500 Volts. Fig. 6.1.2.4 shows the resulting tracking error. Fig. 6.1 2.5 shows the tracking error for a $0.5 \mathrm{~mm}$ offset, which amounts to an average error of less than 25 microns (since the error is random, and small compared to the 100 micron goal for single wire resolution, it is acceptable).

Another important parameter is the wire positioning accuracy based on knowledge of wire tension. The RDT technique requires that wire sag by an amount of about 250 microns be corrected for accurately to enable an overall single wire resolution of 100 micron or less. The tests done at Boston University are summarized in Fig. 6.1.2.6, which shows the set-up and the relevant formulae. The tension in the main length of the wire was determined from the fundamental resonant frequency, $f$. This was determined by passing a sinusoidal current of different frequencies through the wire in the presence of a magnetic field. The wire tension can be determined using the measured resonant frequency, and consequently the wire sag can be calculated (see Fig. 6.1.2.6 ; note that the sag can be determined solely from the resonant frequency). The calculated sags were compared with the actual sags measured with the microscope. Fig. 6.1.2.7 shows a comparison of the actual position of the wire with that determined through the frequency measurements for various wire tensions. Fig. 6.1.2.8 shows the differences of these numbers: a precision of the order of 5 microns is achievable. Fig. 6.1.2.9 shows the differences between the wire tensions $\mathrm{T}_{1}, \mathrm{~T}_{2}$, and T3. Note that the wire tension is typically $10 \%$ lower than the weight, due to friction forces.

Fig. 6.1.2.10 shows the resonant frequency for the 7 different wire tensions. It is important to make sure that the vibration frequency spectra in the GEM experimental area are not such as to induce oscillations in the RDT wires. For comparison, it was observed at BU that large oscillation of the wires occurred at about $10 \mathrm{~Hz}$, reflecting the dominant building vibration frequency there. Thus we expect no problems since the operation of drift tubes will occur at resonant frequencies of about $35 \mathrm{~Hz}$. Fig. 6.1.2.11 shows the additional wire sag induced on a wire in a tube due to electrostatic forces. For $4 \mathrm{kV}$ for example, such sag is only of the order of 10 microns.

The Boston University group has participated in various accelerator and balloonborne experiments using cylindrical drift tubes of various sizes and dial..eters. Among these, the HRS drift tube array, built 12 years ago, offered an excellent opportunity to study wire tension stability over a long period of time. The BU group was fortunate in being allowed to move one of the spare octants of HRS tubes (112 tubes, $3.5 \mathrm{~m}$ long, $2.54 \mathrm{~cm}$ diameter, 50 microns diameter wire) to Boston. They then used the technique described previously to measure tension. The results are shown in Fig. 6.1.2.12. Note that the wire tension, set for $250 \mathrm{~g}$ in 1980, varies typically by no more than several percent for all wires (average tension $248 \mathrm{~g}$, standard deviation of $17 \mathrm{~g}$, and a standard deviation of the wire sag of only 16 microns). 


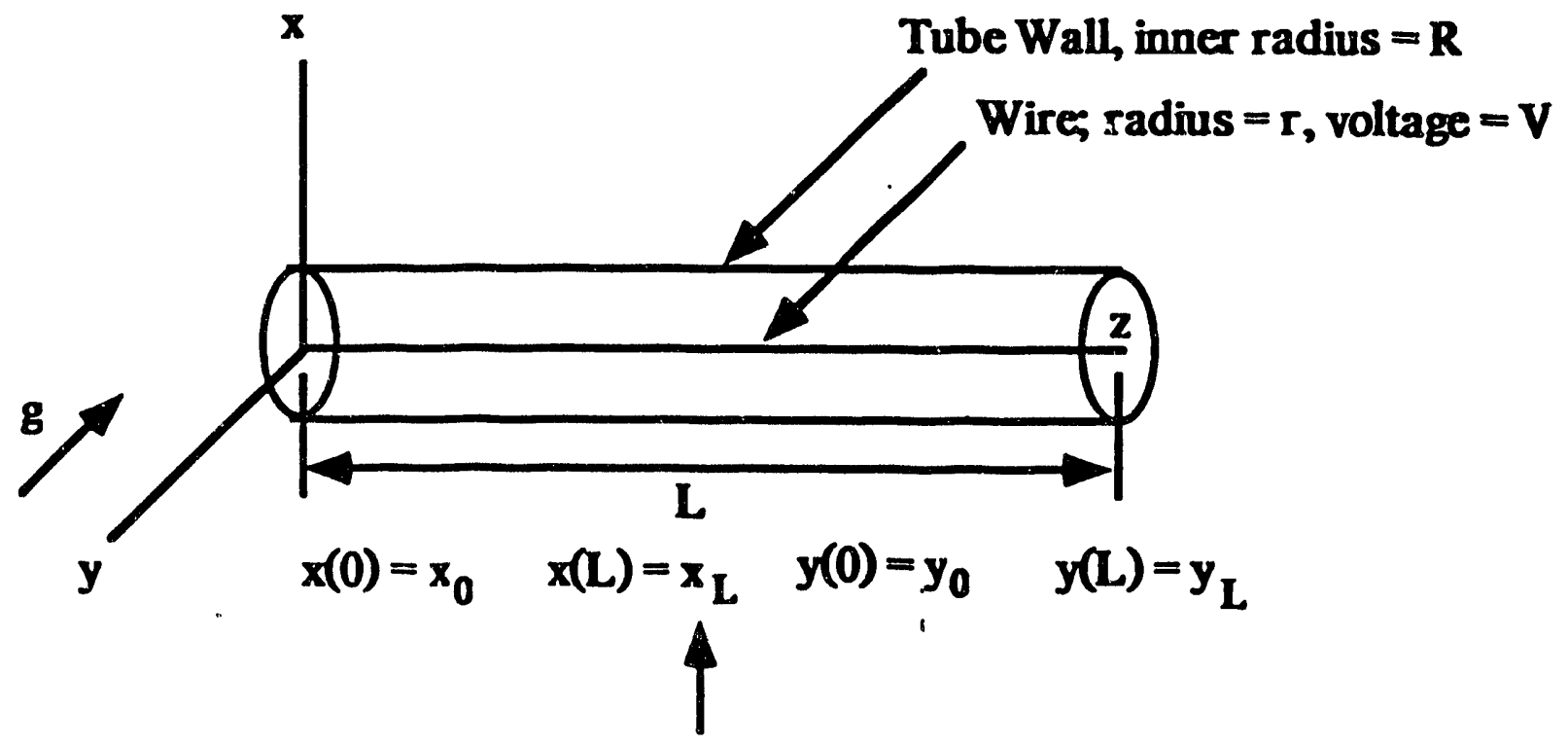

Coordinates of fixed ends of wire, tube center is along $\mathrm{z}$ axis

$$
\begin{gathered}
\stackrel{\rho}{\rho}=\text { mass density of wire } \\
T=\text { tension of wire } \\
K=2 \pi \dot{\varepsilon}_{0} V^{2} /[R \ln (R / r)]^{2} \\
: \\
y_{B}=\rho \pi r^{2} g / K \\
\omega_{1}^{2}=K r T
\end{gathered}
$$

$$
y(z)=\left(y_{0}-y_{g}\right) \cos (\omega z)+y_{g}+\left[y_{L}+\left(y_{g}-y_{0}\right) \cos (\omega L)-y_{g}\right] \sin (\omega z) / \sin (\omega L)
$$

$$
x(z)=x_{0} \cos (\omega z)+\left[x_{L}-x_{0} \cos (\omega L)\right] \sin (\omega z) / \sin (\omega L)
$$

$$
\mathrm{V}(\text { critical })=3611 \mathrm{~V}(\mathrm{~T} / 300 \mathrm{~g})^{0.5}(\mathrm{R} / 2 \mathrm{~cm})(4 \mathrm{~m} / \mathrm{L}) \ln (\mathrm{R} / \mathrm{r})
$$




\section{ELECTRIC FIELD IN DRIFT TUBE CAN BE EASILY CALCULATED PRECISELY}

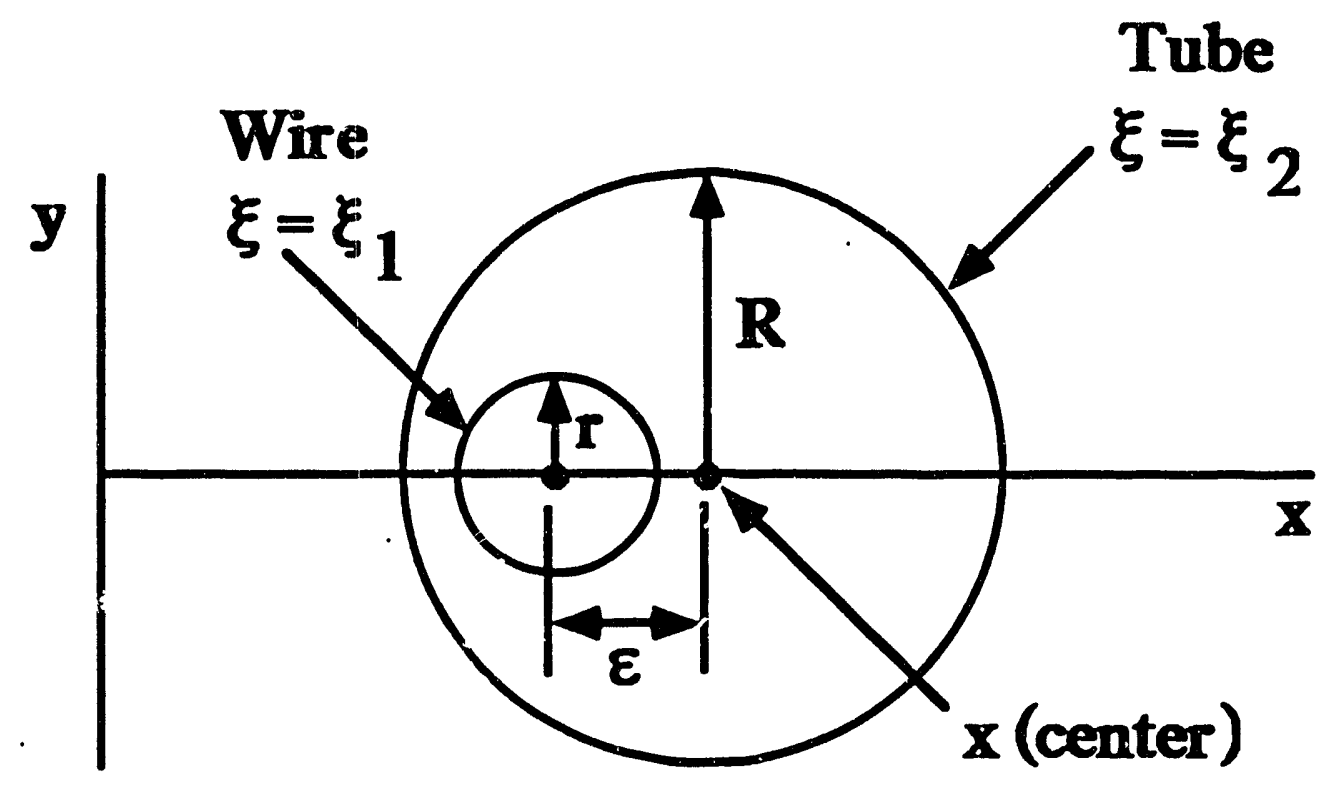

\section{BIPOLAR COORDINATES}

$\mathbf{x}=\mathbf{a} \sinh (\xi) /(\cosh (\xi)+\cos (\theta))$

$\mathbf{y}=\mathbf{a} \sin (\theta) /(\cosh (\xi)+\cos (\theta))$

$\varepsilon=\left(R^{2}+a^{2}\right)^{1 / 2}-\left(r^{2}+a^{2}\right)^{1 / 2}$

$x$ (center) $=a \operatorname{coth}\left(\xi_{1}\right)$

$\mathbf{r}=\mathbf{a} / \sinh \left(\xi_{1}\right)$

$\mathbf{R}=\mathbf{a} / \sinh \left(\xi_{2}\right)$

$E_{\mathbf{X}}=-V(\cos (\theta) \cosh (\xi)+1) /\left[a\left(\xi_{1}-\xi_{2}\right)\right]$

$\mathbf{E}_{\mathbf{y}}=\mathbf{V}(\sin (\theta) \sinh (\xi)) /\left[\mathbf{a}\left(\xi_{1}-\xi_{2}\right)\right]$

Fig. 6.1.2.2 

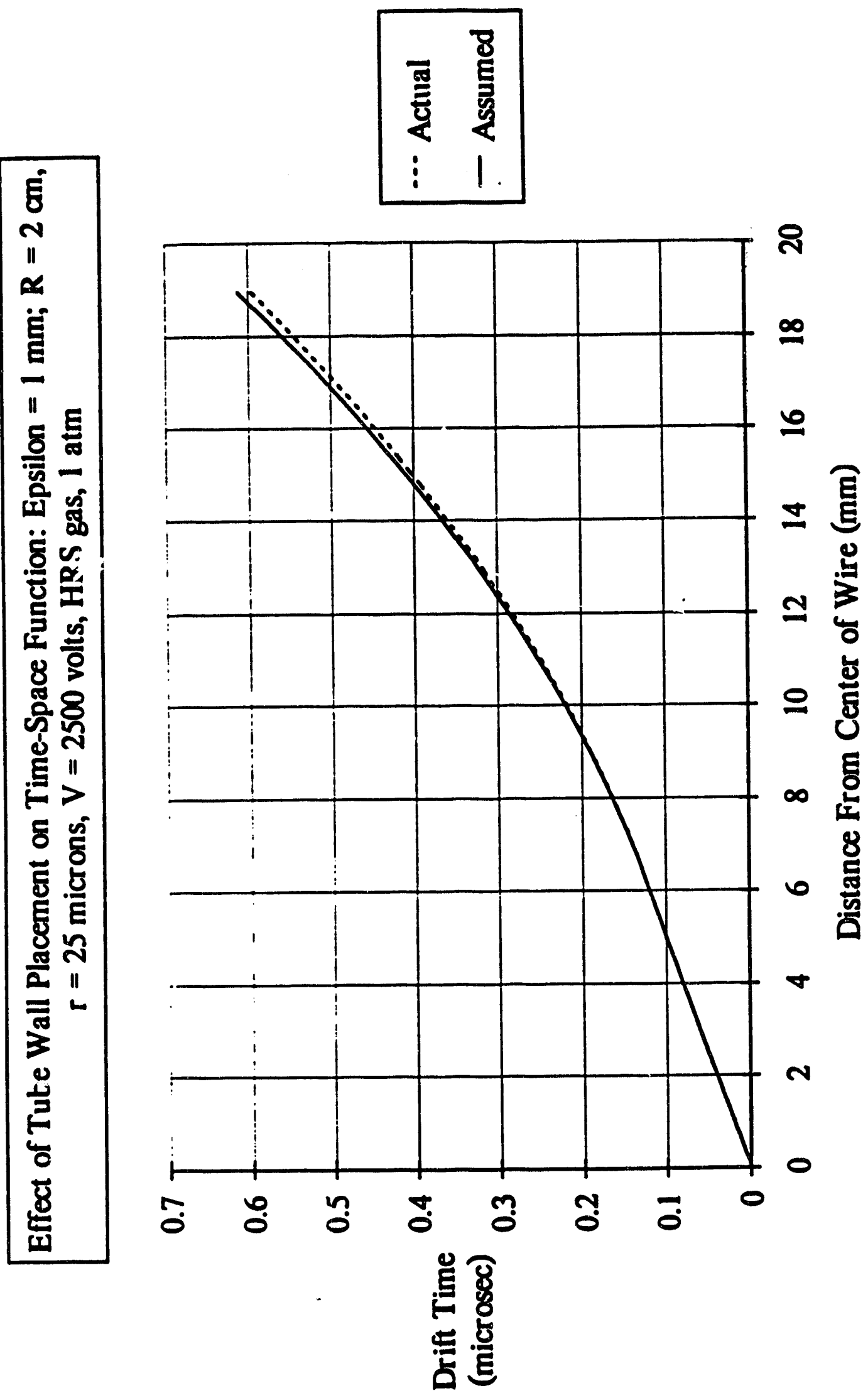

Fig. 6.1.2.3 


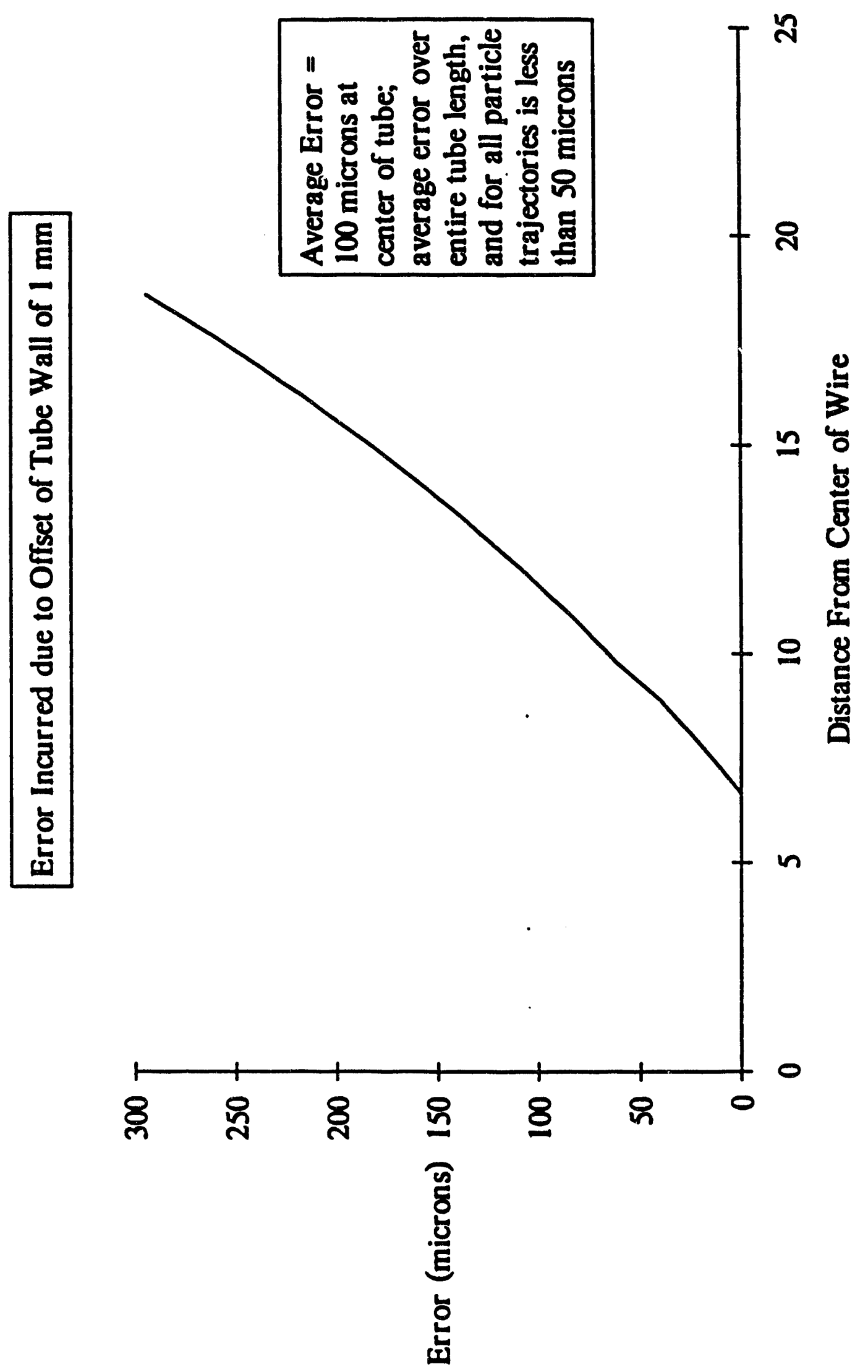

Fị̣. 6.1.2.4 


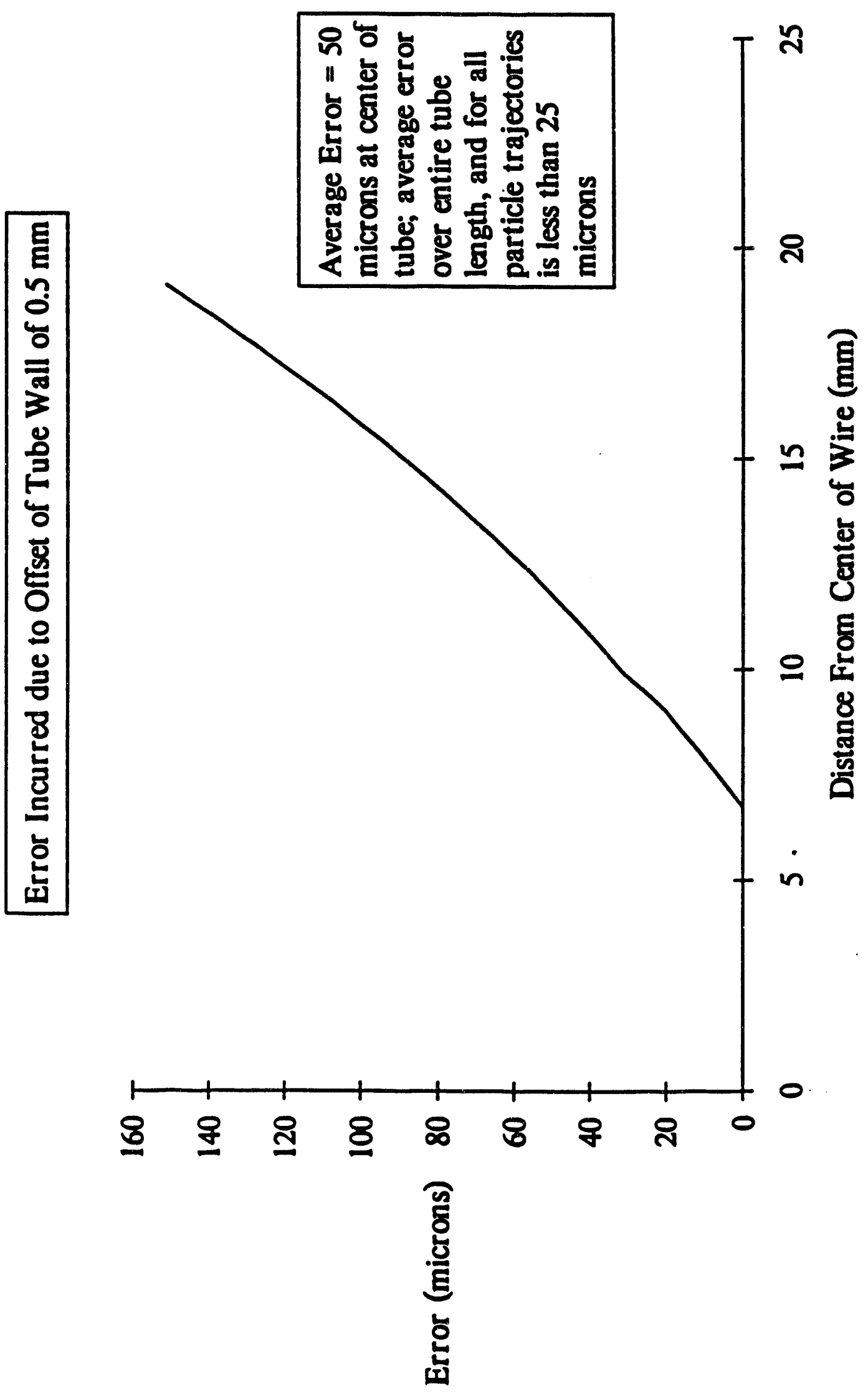

- 


\section{Wire Tests}

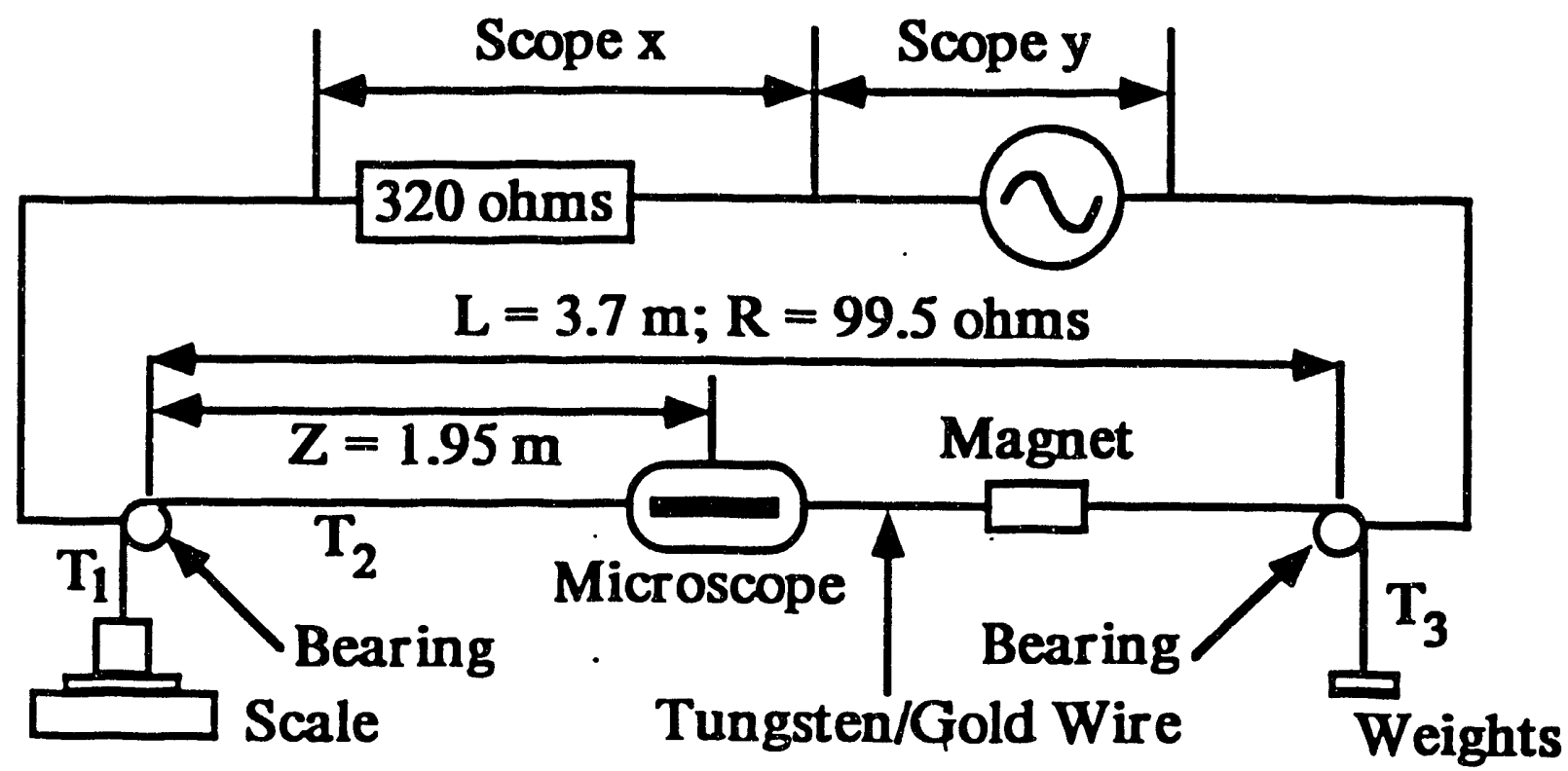

Scope y

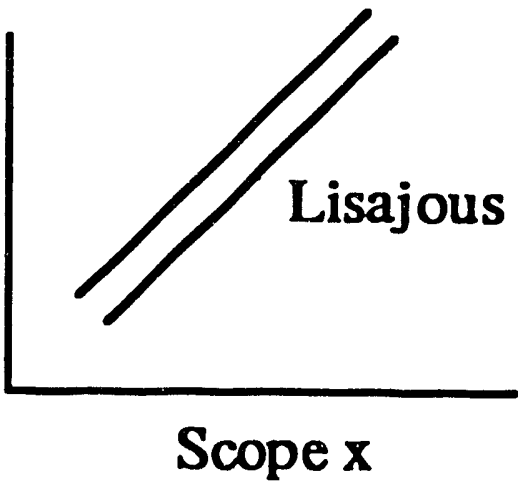

$R=99.5$ ohms implies diameter $=2 r=49.6$ microns $(70 \mathrm{~F})$

S = wire sag

$\rho=$ mass density $=19.3 \mathrm{~g} / \mathrm{cc}$ (gold and tungsten)

$\mathbf{g}=$ gravitational acceleration

$\mathrm{f}=$ fundamental resonant frequency

$T=$ tension in wire

$$
T=\rho \pi r^{2}(2 L f)^{2}
$$

$S=\rho \pi r^{2} g\left(Z^{2}-L Z\right) /(2 T)=g\left((Z / L)^{2}-(Z / L)\right) /\left(8 f^{2}\right)$

NOTE: $S$ depends on $f$ only, and has no additional dependence on $\mathbf{T}$ or $\mathbf{r}$

Fig. 6.1.2.6 


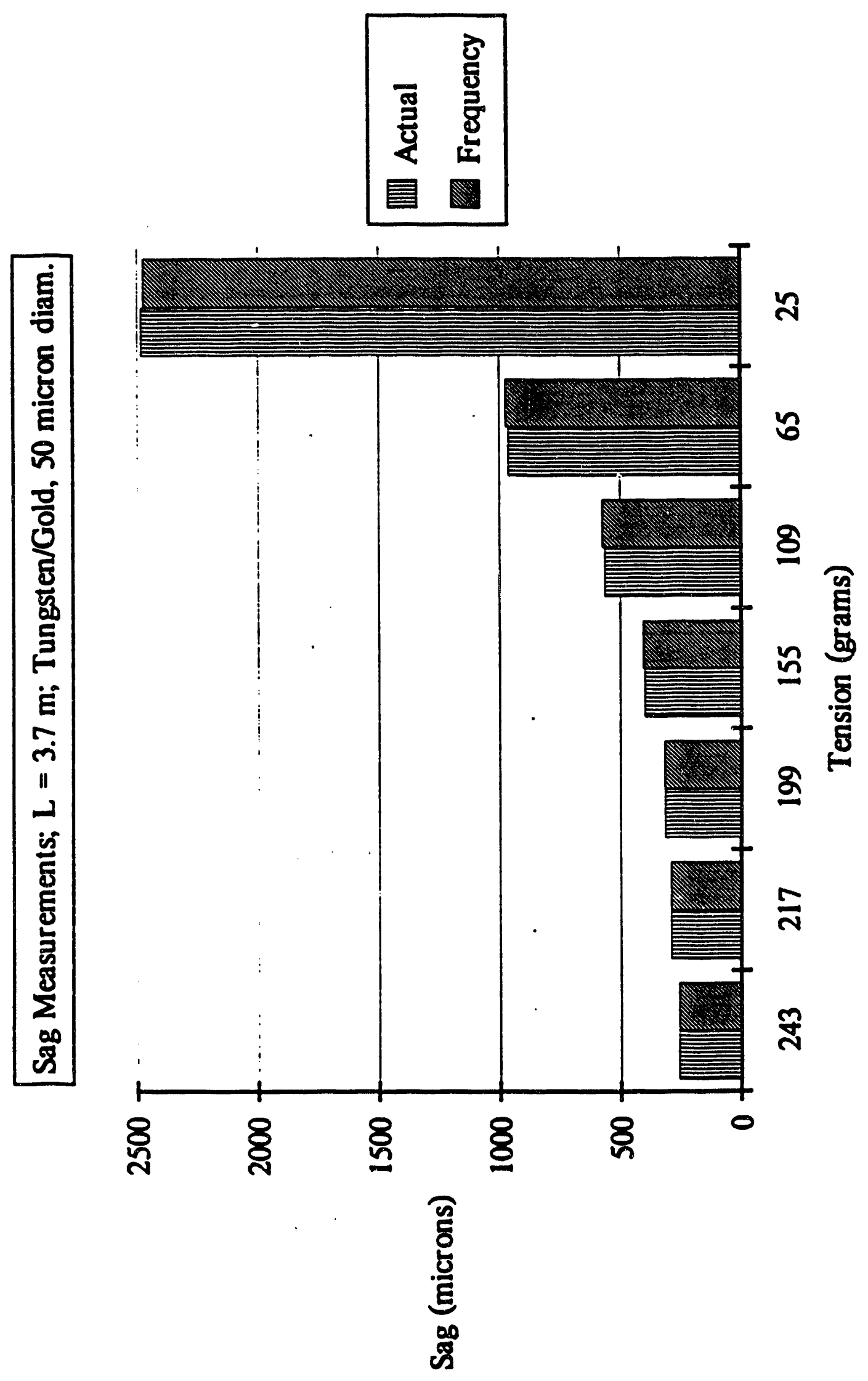

Fiq. 6.1.2.7 


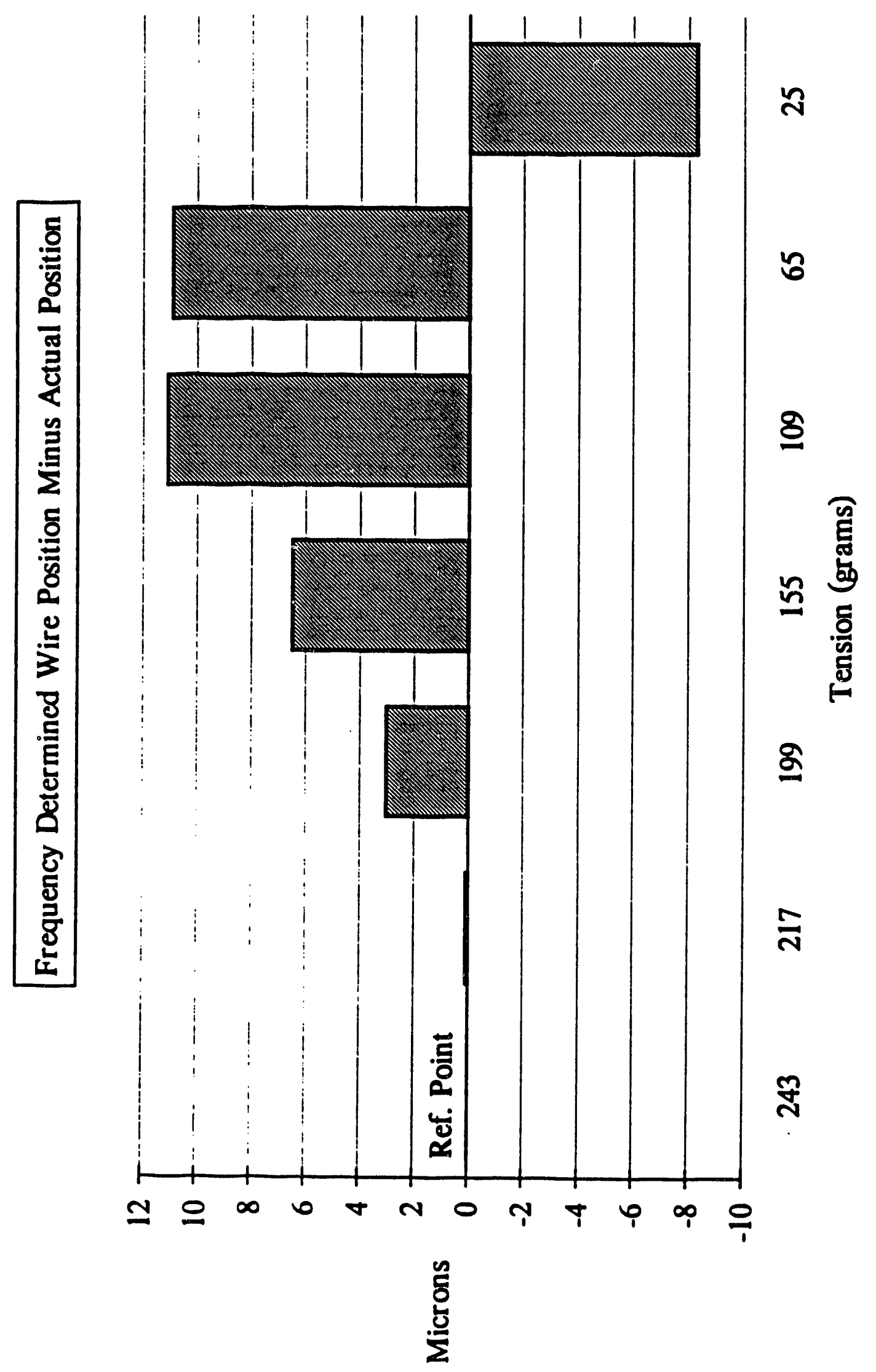

Fig. 6.1.2.8 


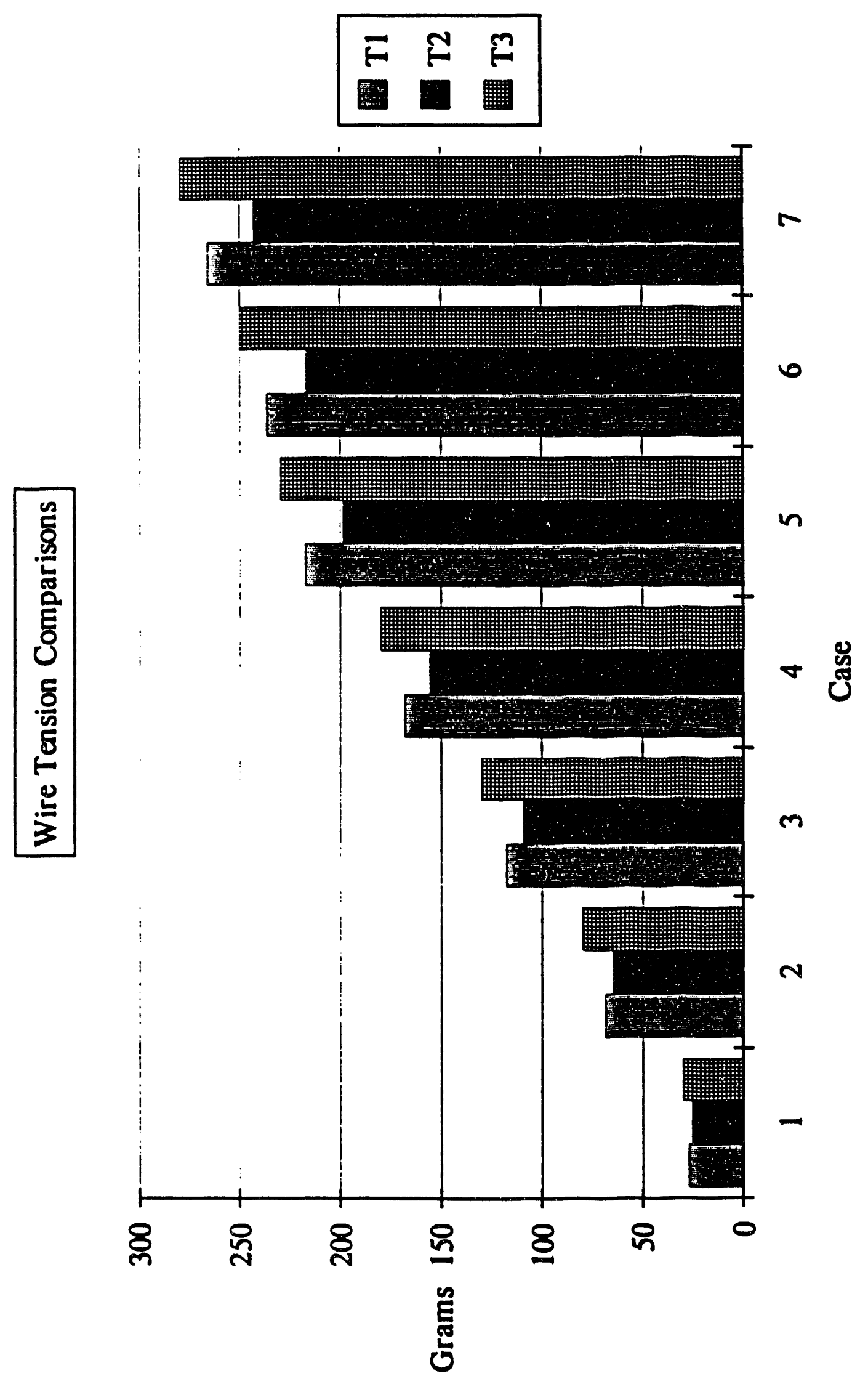

Fiq. 6.1.2.9 


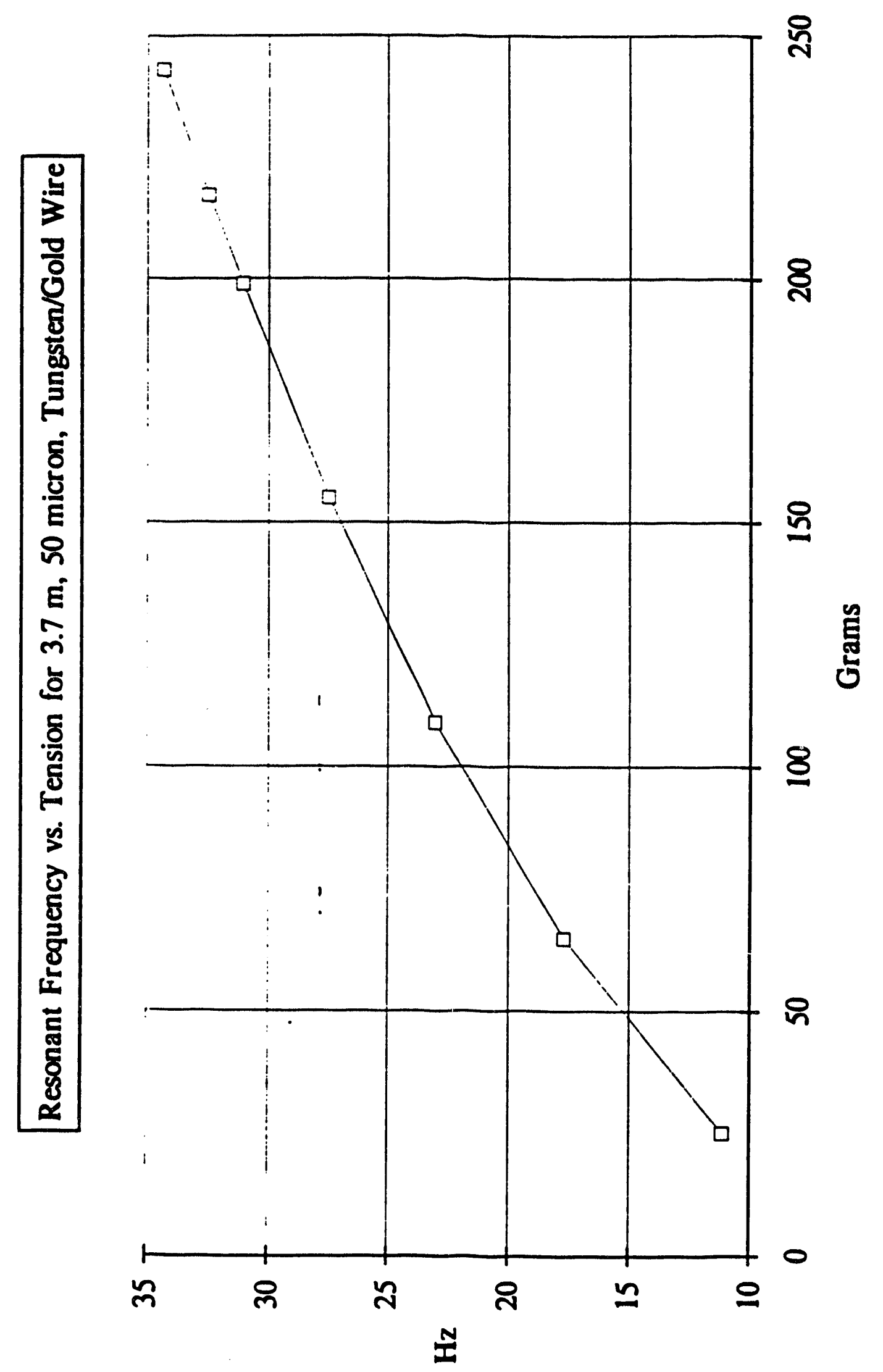

Fị̣. 6.1.2.10 

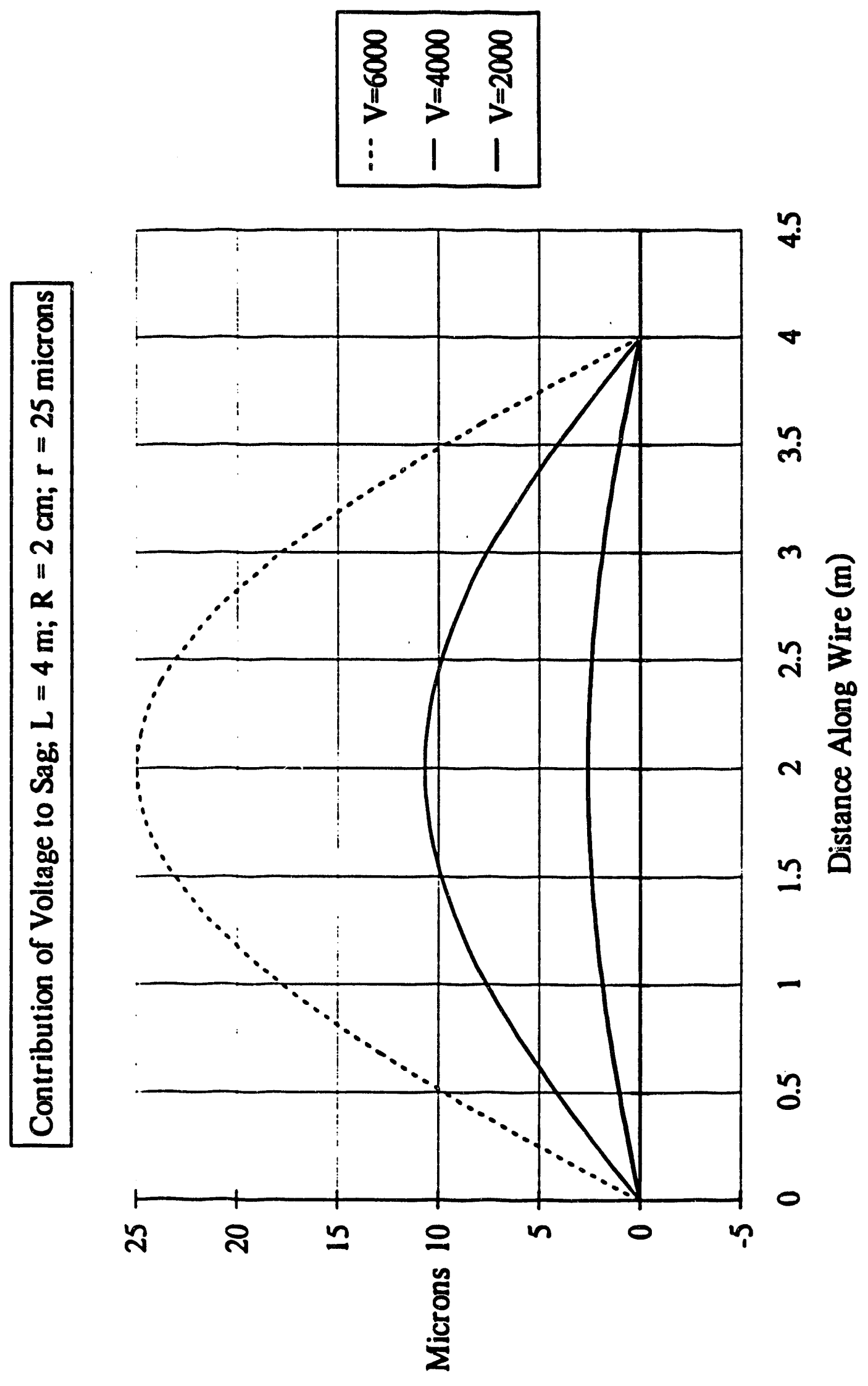

Fị̣. 6.1.2.11 

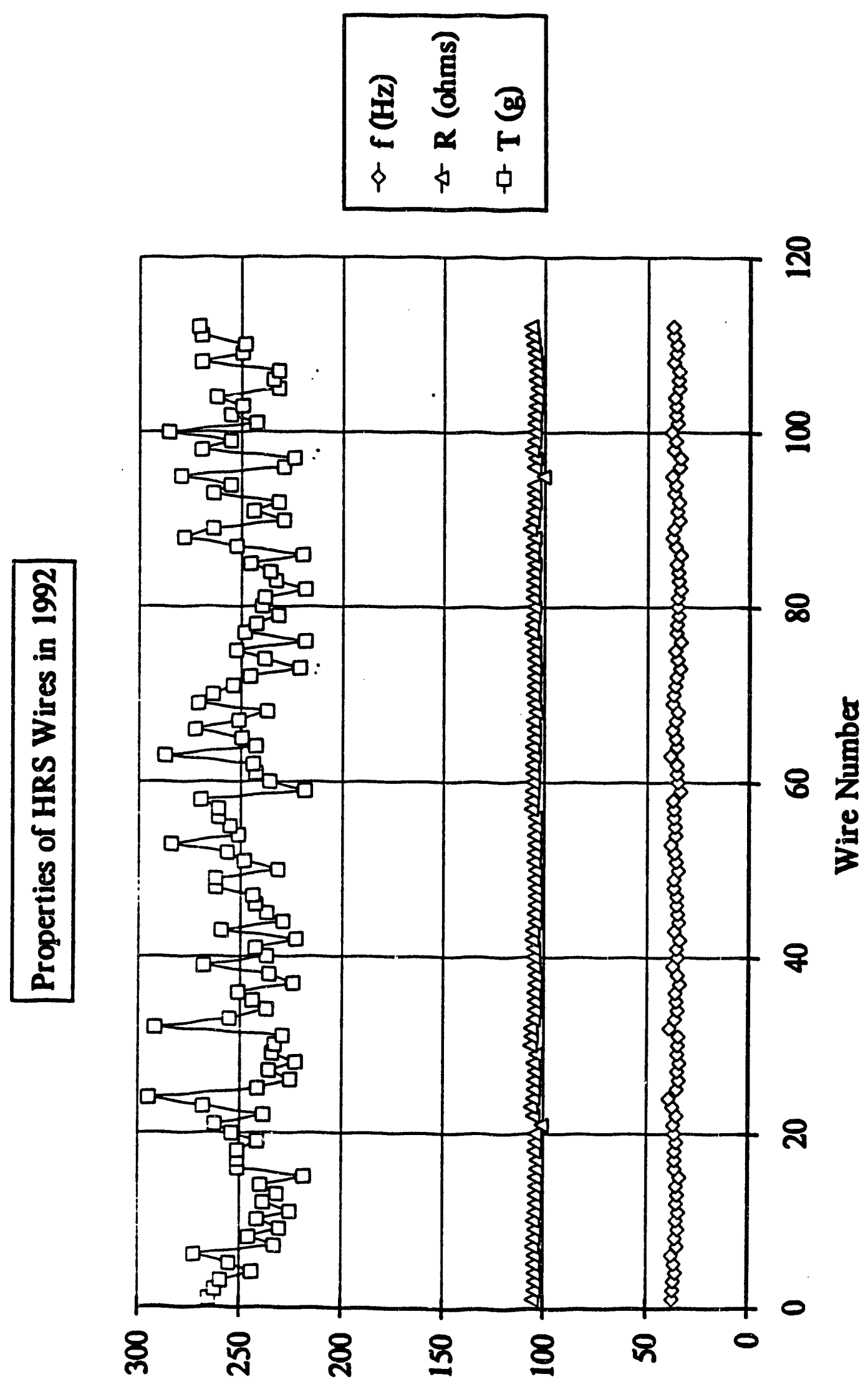

Fị̣. 6.1.2.12 


\section{(6.2) Tests of the RPC Technology:}

The R\&D program had the goal of a complete understanding of the operation and applicability of RPCs to the GEM Muon System. R\&D has focused on studying the existing state-of-the-art as exemplified by the standard Italian design already described, as well as new RPCs using non-standard materials and fabrication methods, with an emphasis on reduced mass, stability and long term performance, and increased rate capability.

\section{Standard Italian and Glass RPC Performance}

MIT and LLNL have now studied a number of different RPC designs, including a 1 $\mathrm{m} \times 2 \mathrm{~m}$ RPC provided by R. Santonico of the University of Rome, glass RPCs, cermet thin film RPCs and RPCs using a number of low resistivity materials such as staticdispersive plastics. Extensive testing of the Italian RPC, a custom built glass RPC, and, very recently, a plastic RPC, has been carried out at MIT, including measurements of efficiency, rise-time jitter, and response to neutrons, and the results of some of these tests are detailed in Appendix A. LLNL has also performed similar measurements on smaller glass RPCs.

In summary, the MIT and LLNL glass RPCs (resistivity $=5 \times 10^{12} \mathrm{ohm}-\mathrm{cm}$ ), with their relatively high bulk resistivity, exhibit a reduced rate handling capability and a reduced efficiency ( $70 \%$ for the MIT RPC with $2 \mathrm{~mm}$ glass and $90 \%$ for the LLNL RPC with 750 micron glass). The Italian Bakelite RPC (resistivity $=1-2 \times 10^{11} \mathrm{ohm}-$ $\mathrm{cm}$ ) exhibits about $95 \%$ efficiency using cosmic rays and a scintillator telescope for triggering. The Italian RPC has a measured saturated counting rate of about 560 $\mathrm{Hz} / \mathrm{cm}^{2}$ compared to a rate of about $50 \mathrm{~Hz} / \mathrm{cm}^{2}$ for the MIT glass RPC $\left(75 \mathrm{~Hz} / \mathrm{cm}^{2}\right.$ for the LLNL glass RPC). On the other hand, these different RPCs agree remarkably well in terms of pulse characteristics, e.g., pulse height, pulse width, rise time and pulse velocity along the strip. !

\section{Trigger Jitter}

Trigger jitter measurements. on the Italian RPC show time jitter on the order of $1 \mathrm{~ns}$. The MIT glass RPC exhibits a larger jitter of about $7 \mathrm{~ns}$ due to the inability to achieve an adequate high voltage across the gas gap as detailed in Appendix A. Trigger jitter measurements have also been performed on plastic RPCs using strontium-90 betas (2.3 MeV endpoint energy). For a $6 \mathrm{~cm} \times 6 \mathrm{~cm} \mathrm{RPC}$ the jitter was measured to be $5 \mathrm{~ns}$.

\section{Neutron Sensitivity}

Neutron sensitivity measurements were made using a strong Cf-252 source. The sensitivity of the RPC to $1-10 \mathrm{MeV}$ neutrons is measured to be $4.8 \times 10^{-3}$ and to $1-10$ $\mathrm{MeV}$ photons to be $6.6 \times 10^{-3}$. 


\section{Alternative RPC Materials for High Rate RPCs}

RPCs have been fabricated at LLNL using sputtered resistive cermet thin films on thin glass or plastic substrates. We have demonstrated that Cermets can function as RPCs, however the long term aging characteristics of these thin films is not clear. Indications are that the films are not strongly bonded to the substrate and are subject to sputtering effects because of ion bombardment. Alternative materials to Bakelites have been explored, concentrating on static-dispersive plastics with low bulk resistivities in the range $10^{8}-10^{11} \mathrm{ohm}-\mathrm{cm}$. We have successfully demonstrated RPCs with a number of different plastics. Five $0.5 \mathrm{~m} \times 0.5 \mathrm{~m} \mathrm{RPCs}$, and one $1.2 \mathrm{~m} \mathrm{x}$ $2.4 \mathrm{~m}$ RPC have been built using low resistivity plastic materials. All RPCs are single dimension read-out with $1.3 \mathrm{~cm}$ strips, and one of the small RPCs, is built with 2 dimensional read-out. Table 6.2.1 summarizes the properties of

Table 6.2.1

RPC Resistive Materials Properties

Material Thickness (cm) Bulk Resistivity (W-cm) Arc Resistivity (W- $\mathrm{cm}^{2} 2$

$\begin{array}{llll}\text { MIT mirror glass } & 0.300 & 5.00 \times 10^{12} & 1.50 \times 10^{12} \\ \text { LLNL mirror glass } & 0.066 & 4.90 \times 10^{12} & 3.23 \times 10^{11} \\ \text { Kodak projector glass } & 0.123 & 6.42 \times 10^{11} & 7.89 \times 10^{10} \\ \text { Italian RPC Bakelite } & 0.200 & 1.00 \times 10^{11} & 2.00 \times 10^{10} \\ \text { LLNL Bakelite } & 0.161 & 4.50 \times 10^{9} & 7.24 \times 108 \\ \text { Abstat-M310 plastic } & 0.072 & 5.78 \times 10^{9} & 4.16 \times 10^{8} \\ \text { Abstat-M310 plastic } & 0.060 & 5.78 \times 10^{9} & 3.47 \times 10^{8} \\ \text { MiTech-411 plastic } & 0.090 & 2.03 \times 10^{9} & 1.83 \times 10^{8} \\ \text { MiTech-411 plastic } & 0.030 & 2.03 \times 10^{9} & 6.19 \times 10^{7} \\ \text { Corning 0211 glass } & 0.056 & 6.70 \times 10^{7} & 3.75 \times 10^{6} \\ \text { Boron film } & 0.0001 & 1.00 \times 10^{6} & 1.00 \times 10^{2}\end{array}$

a number of materials used to make RPCs, including Bakelites and glasses.

We have measured the saturated counting rates from two different static-dispersive plastics, MiTech-411 and Abstat-M310. For the case of the MiTech-411 plastic, our SPICE model predicts a saturated counting rate capability of about $2.3 \times 10^{4} \mathrm{~Hz} / \mathrm{cm}^{2}$. We have assembled a number of RPCs with this material and in fact measure a count rate of approximately $1.5 \times 10^{4} \mathrm{~Hz} / \mathrm{cm}^{2}$. Fig. 6.2 .1 plots the counting rate versus high voltage for an RPC made with MiTech-411 plastic. This rate has not been determined to be saturated and further work is in progress to determine the absolute saturated count rate of the RPC with this very interesting material.

This count rate is substantially larger than any other RPC materials measured to date, and constitutes a significant improvement in the state of the art. In addition, 


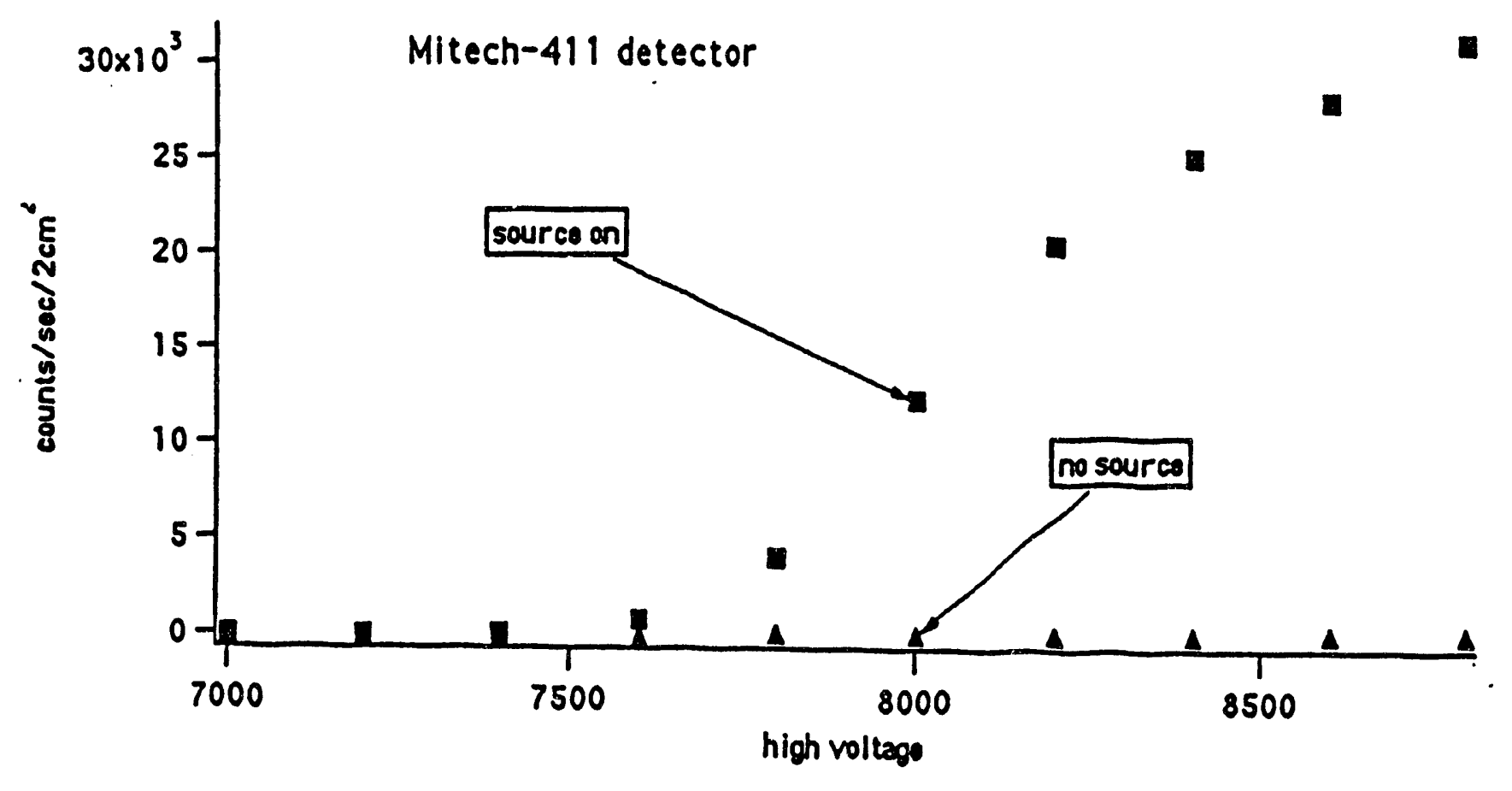

Fig. 6.2.1 
the Abstat M310 plastic has a significantly higher dielectric strength than the PVCbased MiTech-411 plastic. Large area RPCs have been constructed using thinner plastic sheets than those used initially (500 microns versus 720 microns), which could potentially give an increase of 5-10 times the rate of the earlier versions of the plastic RPCs. Measurements of efficiency of large area plastic RPCs show that they operate at or near the full geometric efficiency expected, about $98 \%$. Also, systematic studies of gas mixtures on RPC performance (plateau voltage, efficiency, noise, pulse height, etc.) have been performed for large area plastic RPCs.

\section{Lifetime Testing of the Italian RPC}

Lifetime (aging) tests have also been performed using the talian $1 \mathrm{~m} \times 2 \mathrm{~m}$ Bakelite RPC. We chose an area of this RPC approximately $5 \mathrm{~cm} \times 10 \mathrm{~cm}$ which was irradiated with a radioactive source. The counting rate on this area was approximately 250 $\mathrm{Hz} / \mathrm{cm}^{2}$. We placed a cosmic ray scintillation counter telescope which had approximately the same $5 \mathrm{~cm} \times 10 \mathrm{~cm}$ area over the chosen area of the RPC. We chose the RPC region so that one of the PVC spacers was contained in the area.

We define one SSC year (at a luminosity of $10^{33}$ ) to be $2 \times 10^{7}$ counts per square centimeter. This is the expected neutron interaction rate at the $10^{33}$ luminosity. We accumulated $16.4 \times 10^{7}$ counts per square centimeter in our test, which is equivalent to 8.2 SSC standard years. The data is shown in Fig. 6.2.2.

Before irradiation the chosen area had an efficiency of $93.6 \pm 3 \%$. The fact that the measured efficiency is not about $97 \%$ is a reflection of the mismatch between the cosmic ray hodoscope and the RPC area. After an irradiation equivalent to $8.2 \mathrm{SSC}$ years of operation, we measured the efficiency of this area to be $92.4 \pm 3 \%$. As can be seen from Fig. 6.2.2, within the 3\% errors, we find no change in efficiency for the equivalent $8.2 \mathrm{SSC}$ year exposure at a luminosity of $10^{33}$.

\section{Measurements of RPC-induced Noise on RDT Systems}

A number of measurements of RDT sensitivity to RPC signals have been made at the Texas Test Rig (TTR) at SSCL. Measurements have been made using both the Italian $1 \mathrm{~m} \times 2 \mathrm{~m}$ RPC installed in the TTR and $0.5 \mathrm{~m} \times 0.5 \mathrm{~m}$ plastic RPCs. Both MSU $(4 \mathrm{~cm}$ diameter) and Dubna ( $2.5 \mathrm{~cm}$ diameter) RDT systems have been measured as well as the UH cathode strip chambers. Measurements of signals induced on RDT wires were made by examining the signals off the wires on an oscilloscope while triggering on RPC signals. The MSU RDT system contained amplifiers with $\times 1000$ gain, whereas the Dubna RDT system was unamplified. The RPC was placed approximately $1 \mathrm{~m}$, and $2 \mathrm{~m}$ from the MSU and Dubna RDTs, respectively. Under these conditions, no induced signals were observed at a level of 5 microvolts and 2 millivolts for the MSU and Dubna RDTs, respectively. Also, no induced signals were observed on the UH CSC system at a level of 5 microvolts. 


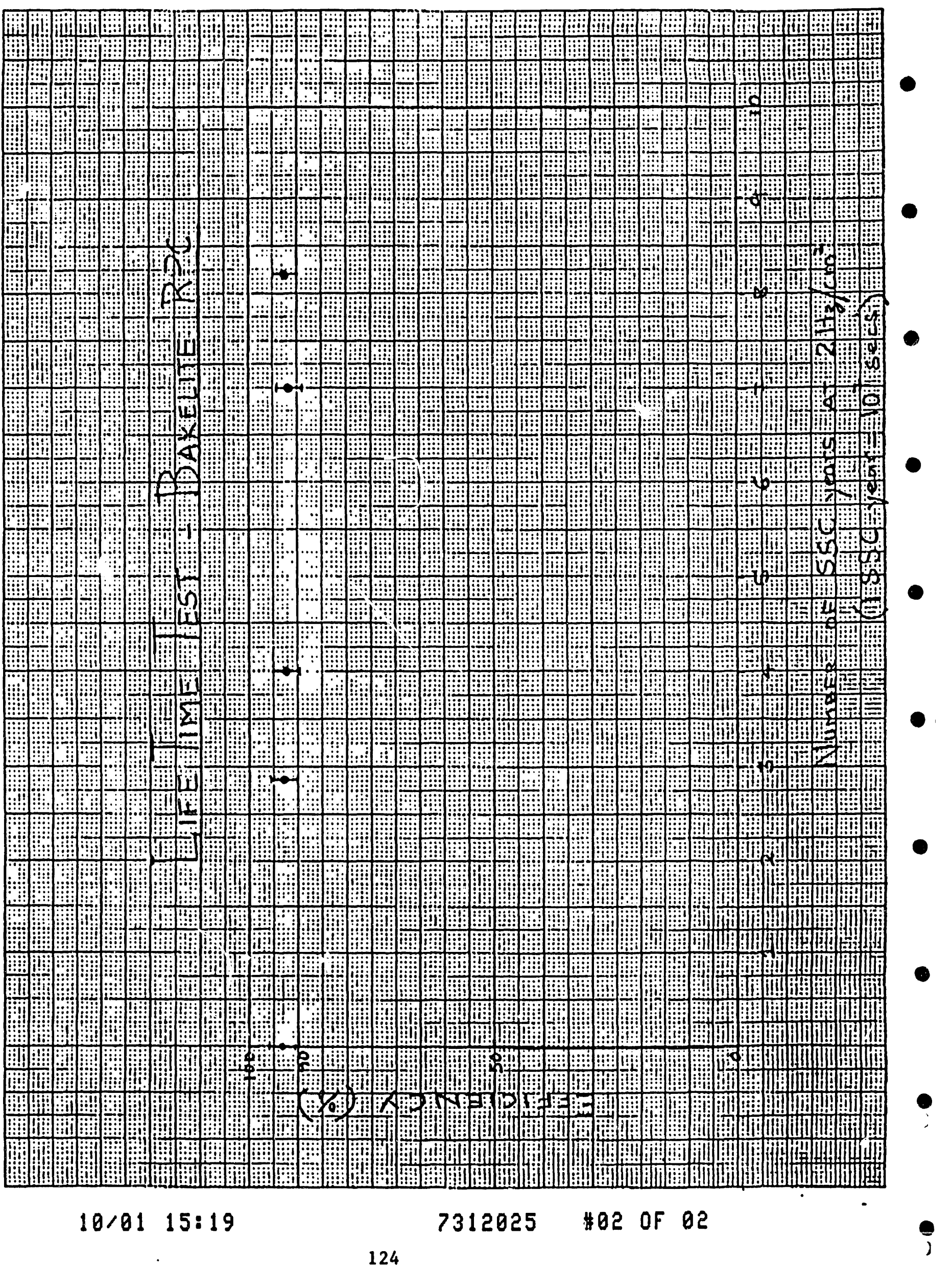


Another measurement was made by measuring the effect of RPC operation on RDT singles rates. In the case of the MSU RDTs, no increase in singles rate was observed for either the Italian RPC or the LLNL RPC as these RPCs had their voltages increased. In this case the singles rates of the RPCs increased by three and five orders of magnitude, respectively, as their voltages were increased from $6 \mathrm{kV}$ to $8 \mathrm{kV}$. Similar measurements were made for the Dubna RDT and the UH CSC for the Italian RPC only. Again, no increase in singles rates were observed for a wide range of RPC operating voltages. 


\section{(7.0) Pipeline Muon Trigger based on RPCs}

The basis for the proposed muon trigger is the resistive plate technology. The properties of this technology are:

1. Pulse rise time jitter $\leq 1.4$ nanoseconds.

2. Pulse full width, half height $\leq 20$ nanoseconds.

3. Pulse height @ 500 millivolts into 50 ohms.

A PWC instrumented with the same segmentation will provide a trigger in essentially the same manner.

\section{SIMPLIFIED LOGIC FOR THE BARREL TRIGGER}

\section{A. Introduction}

The barrel trigger is based on 250 megahertz shift register logic. One can adjust cable lengths and gate widths so that all relevant signals from the non-bend strips and the bend strips can be loaded synchronously into shift registers. The outputs of these shift resistors can be put into simple logic to form the trigger and beam crossing tag. The trigger is formed in 100 nanoseconds. At a neutron flux of $10^{5}$ Hertz per square centimeter per second the accidental trigger rate in the barrel is about 29 Hertz. In the end cap the accidental rate is even less. The dead time is about $6 \%$ for a gate width of 100 nanoseconds.

\section{Geometry of an RPC Barrel Plane}

In Fig. 7.0.1 is the typical $X$ and $Y$ strip lay out. The crucial thing to note is that a hit anywhere on the plane generates signals on an $X$ strip and $Y$ strip that arrive at the collection point in coincidence. Another point to note is that half the bend plane strips are in coincidence with the lower half of the non-bend plane strips while the other half of the bend plane strips are in coincidence with the upper half of the nonbend plane strips. This division has two virtues, the first it reduces the time resolution in tagging the beam crossing by about a factor two and it also reduces the ambiguity of double hits by a factor two. Note that this is achieved without increasing the electronic channel count. These points will be discussed in detail in later sections of this paper.

It should be noted that each RPC super layer consists of three identical RPC counters with the requirement that two out of three strips fire when a track passes through the super layer. This increases the low rate efficiency of the RPC trigger system from about $90 \%$ to essentially $97 \%$ without increasing the electronic channel count. It does, however, increase the total number of signal cables and electronic inputs. This is discussed in detail in Appendix B. 


\section{B. Geometry of an RPC Sector}

Fig. 7.0.2 is a simplified sketch of the three RPC super layer chambers that form an RPC sector.

As can be seen from Fig. 7.0.2, there is an outer RPC super layer which we will label SL3, a central RPC super layer which we will label SL2, and an inner RPC super layer which we will label SL1 in accordance with the GEM Base Line 1 notation.

The non-bend strips form projective towers with respect to the interaction point. The non-bend plane strip widths are:

$\begin{array}{ll}\text { SL1 }(A \text { and B) } & =3.9 \mathrm{~cm} \\ \text { SL2 }(A \text { and B) } & =6.5 \mathrm{~cm} \\ \text { SL3 }(A \text { and B) } & =8.9 \mathrm{~cm}\end{array}$

The number of non-bend plane chambers per sector are given below:

Non-bend plane channel count:

$\begin{array}{ll}\text { SL1 } & =166 \text { channels } \\ \text { SL2 } & =166 \text { channels } \\ \text { SL3 } & =166 \text { channels }\end{array}$

All bend plane strips are each $1.3 \mathrm{~cm}$ wide. The number of bend plane strips per sector are given below:

Bend plane channel count:

SL1 = 345 channels

SL2 $=552$ channels

SL3 $=762$ channels

The total number of electronic channels we have for each sector is:

Non-bend plane $=498$ electronic channels

Bend plane $\quad=1659$ electronic channels

As we stated above, note, that we have more cables and connectors than we have electronic channels.

The total number of electronic channels is 69,024 .

\section{Simplified Trigger and Beam Crossing Logic}

The bend plane logic for the barrel is the most difficult to work out. Hence we will discuss this case first. It can be shown that with proper choice of cable lengths 
between SL1 and SL3; and SL2 and SL3, corresponding strips in the three super layers can be placed in coincidence using predetermined delays as inputs to the shift registers. This is discussed in Appendix B.

The proposed trigger is a three layer trigger. Due to the multiple scattering in the calorimeter, a number of possibilities must be considered. Fig. 7.0.3 illustrates this.

For each pair of strips in SL3 and SL1 there is a range of strips in SL2 that determine the momentum of the track.

For a given strip in SL3 and a given strip in SL1 there is a shift register logic for forming a trigger.

A trigger can be formed under computer control. The trigger momentum for each strip in SL3 that forms a trigger is chosen by which strips in SL1 are selected, and then for each selected strip in SL1 which strips in SL2 are selected. Fig. 7.0.4 illustrates the situation for a $20 \mathrm{GeV} / \mathrm{c}$ track.

As a specific example we consider a trigger for momentum greater than $30 \mathrm{GeV} / \mathrm{c}$. In this case, for each sign of charge, three strips in SL1 must be selected for each strip in SL3. In addition, for each strip selected in SL3, two strips must be selected for SL2. If we consider both possible charges, then we activate six similar sets of shift register logic. We define the following symbols:

SL3i

SL1j $\quad(j=1$ to 6$)$

SL2k ( $k=1$ to 3$)$
The strip in super layer three that has been struck

The strip in super layer one that has been struck. The six possible strips are determined by the 30 $\mathrm{GeV} / \mathrm{c}$ sagitta at super layer 1 with respect to an infinite momentum track that passes through SL3i and the interaction point and the multiple scattering due to a $30 \mathrm{GeV} / \mathrm{c}$ muon passing through the calorimeter.

The strip in super layer two that has been struck. The three strips are determined by the $30 \mathrm{GeV} / \mathrm{c}$ sagitta at super layer 2 with respect to an infinite momentum track that passes through strips SL3i and SL1j.

The shift register logic for this situation is shown in Fig. 7.0.4.

Every four nanoseconds the first eight shift registers are loaded with the eight bit address of strip SL3i. The next shift register is the input of SL3i, which we have assumed has been hit. The next 3 shift registers contain the hit pattern in SL2. The following 8 shift registers contain the eight bit address of SL1j. The following shift 
register is the input of SL1j, which we have assumed has been hit. The last shift register is the local trigger flag for this logic circuit. Note that we have allowed five clock ticks to fan out all strips to their required logic. Clock ticks 6 and 7 form an or of the SL2 strips. Clock ticks 8 and 9 form a triple coincidence between SL3i, SL1j, and the or of the SL2 strips. This is the actual trigger. Clock ticks 10 and 11 form a fanout of the trigger. Clock tick 12 sets the local trigger flag and feeds the pipelined 256 or circuit, one for each strip of SL3. Clock ticks 13 through 16 form this or. The output at clock tick 18 is the bend plane master trigger. Clock tick 18 and 19 form the coincidence between the bend plane master trigger and the nonbend plane master trigger. The formation of the non-bend plane master trigger is discussed later in this paper. Clock ticks 20 and 21 open a 64 line data bus. Clock ticks 22 and 23 activate the 64 line data bus. Clock ticks 24 and 25 load the data bus. Note that the local trigger addresses the bus in the usual memory fashion. Clock ticks 24 and 25 send the trigger and the complete information to the second level trigger. We use a 64 bit data bus so that we can transfer 22 bits of bend plane and 42 bits of non-bend plane information. Hence at the end of 25 clock ticks we have the decoded addresses of the SL3 and SL1 hit strips and the SL2 hit strips. Tine same is true for the non-bend plane. The non-bend plane also furnishes the clock time of the beam crossing. The complete trigger is accomplished in 100 nano seconds.

Triggers for $20 \mathrm{GeV} / \mathrm{c}$ and $10 \mathrm{GeV} / \mathrm{c}$ can be produced in similar fashions. By including appropriate gates, which are under computer control, one can select the desired trigger.

\section{Non-Bend Plane Trigger Logic and Beam Crossing Tag}

Fig. 7.0.5 shows the geometry of the non-bend plane. The projective strip design has been optimized to account for multiple scattering.

As indicated in Fig. 7.0.5 for each strip in SL3 there is only one strip in SL2 and three possible hits in SL1. Hence only strips in SL1 are shared by other logic. Each strip in SL1 is fanned out to three logic circuits.

For the non-bend plane, the logic is the same for all momentum. Also for the nonbend plane we only need the 8 bit address of SL3i. SL2j has the same address. The SL1k strips have addresses one less than SL3i, the same as SL3i and one more than SL3i. As we have noted earlier each non-bend strip is effectively divided in half. The uncertainty in time is therefore given by:

$$
\begin{aligned}
& \text { SL1 }=1.485 / 2 \times 5=3.7 \text { nanoseconds } \\
& \text { SL2 }=2.390 / 2 \times 5=6.0 \text { nanoseconds } \\
& \text { SL3 }=3.296 / 2 \times 5=8.3 \text { nanoseconds }
\end{aligned}
$$


Since we have a four nanosecond clock and a 1.5 nanosecond pulse rise time jitter, all three super layers can uniquely determine the beam crossing time. However, since we will be using cable and electronic delays to place the relevant strips in coincidence, only one superlayer can be kept in absolute synch with the beam crossing time. We chose SL1 as the beam crossing timing superlayer. The gate widths of superlayer 1 channels will be four nanoseconds, while the gate widths of the other two superlayers will be larger. This is discussed in Appendix B. Fig. 7.0.6 is a sketch of the non-bend plane logic.

The first shift register indicates whether or not the hit in SL3 is on the lower half of SL3i. In this example we assume the lower half of SL3i was hit. The second shift register indicates whether or not the upper half of SL3i was hit. The next 8 shift registers contain the eight bit address of SL3i. The next shift register indicates whether or not SL3i was hit. In this example we assume SL3i was hit. The next three shift registers contain the hit pattern in SL1. In this example we assume only SL( $i+1)$ was hit. The following 10 shift registers contain the 10 least significant bits of the master beam crossing clock which is loaded every clock tick. The next 6 bits contain the fixed, known delay time from strip SL2i to the master clock. This includes all cable delays and flight time delays from the interaction point. Any delays to the master beam clock from the interaction point can be included in these six bits. The next 10 shift registers contain the beam crossing time. The final shift register contains the non-bend plane trigger.

On clock tick 1 all data is loaded into the 42 shift registers. There is no fan out delay as there is in the bend plane trigger. On clock ticks 2 and 3 we perform the subtraction between the clock 10 bits and SL2i Dt six bits. Also on clock ticks 2 and 3 we perform the or between SL1 ( $i-1)$, SL1 ( $i)$, and SL1 $(i+1)$. On clock tick 4 we deposit the ten bit beam crossing time. Hence in four clock ticks we have the unique beam crossing time. On clock ticks 4 and 5 we form the and between SL3i, SL2 $i$ and the or of the three strips centered on SL1i. This forms the non-bend plane trigger. Hence in 5 clock ticks we have the non-bend plane trigger. On clock ticks 6 and 7 we fan out the non-bend trigger to the trigger bit and to the 166 fan in system. On clock ticks 8 and 14 we generate the non-bend plane trigger. Clock tick 16 - 18 places the master non-bend trigger in coincidence with the master bend plane trigger. Clock ticks 19 to 25 places the non-bend plane information on the 64 bit bus to the level 2 trigger. As indicated in the discussion of the bend plane trigger, the complete muon trigger is formed in 25 ticks or 100 nanoseconds.

It should be noted that the beam crossing tag is now at this level with a maximum error of $3.7+4.0+4=11.7$ nanoseconds. The 3.7 comes from the effective strip length of SL1, the 4.0 is the clock period and the 4 is the three sigma error of the rise time jitter. This can be verified to within a maximum error of 5 nanoseconds at the second level.

\section{END CAP MUON TRIGGER}


Fig. 7.0.7 is the calculated sagitta including the energy loss in the calorimeter for the GEM muon system for different transverse momenta. We see from Fig. 7.0.7 that the sagitta at $16^{\circ}$ is about $6 \mathrm{~mm}$ for $50 \mathrm{GeV} / \mathrm{c}$ transverse momentum. Therefore to resolve $50 \mathrm{GeV} / \mathrm{c}$ in the endcaps we must choose our strip width for the region $16^{\circ}$ to $29^{\circ}$ to be $6 \mathrm{~mm}$. In the region of $9.75^{\circ}$ the sagitta is roughly 4.4 millimeters for 50 $\mathrm{GeV} / \mathrm{c}$ transverse momentum. Thus we must take our strip width to be $4.4 \mathrm{~mm}$ in the region between $9,75^{\circ}$ and $16^{\circ}$. The performance of the trigger with this segmentation is discussed in a later section.

For the non-bend plane, the strip width has to be consistent with multiple scattering and the precision we wish to know the non-bend coordinate. For the barrel, SL2 determines the non-bend coordinate and that was chosen as $6.5 \mathrm{~cm}$ which about matched the multiple scattering requirement. For the end cap it seems $6.5 \mathrm{~cm}$ will match the requirement for the non-bend plane. It also seems that the 6.5 $\mathrm{cm}$ will also about match the multiple scatter reqquirement. Hence we will choose a tower geometry with the width at SL2 $=6.5$ centimeters. Table 7.1 lists the strip widths and the number of strips for the six chambers in the end cap. 
Strip Information for the End Caps

SL3 Non-Bend Plane

CHAMBER STRIP

WIDTH NUMBER STRIPS

$29^{\circ} \quad 93 \mathrm{~mm}$

$16^{\circ} \quad 93 \mathrm{~mm}$

SL2 Non-Bend Plane

CHAMBER STRIP

WIDTH NUMBER STRIPS

$29^{\circ} \quad 65 \mathrm{~mm}$

$16^{\circ} \quad 65 \mathrm{~mm}$

SL1 Non-Bend Plane

CHAMBER STRIP

WIDTH NUMBER STRIPS

$29^{\circ} \quad 38 \mathrm{~mm}$

$16^{\circ} \quad 38 \mathrm{~mm}$
WIDTH

40

40
26

WIDTH

40

26

WIDTH

40

26
SL3 Bend Plane

NUMBER STRIPS STRIP

$6 \mathrm{~mm} \quad 583$

$4.4 \mathrm{~mm} \quad 486$

SL2 Bend Plane

NUMBER STRIPS STRIP

$6 \mathrm{~mm} \quad 409$

$4.4 \mathrm{~mm} \quad 320$

SL2 Bend Plane

NUMBER STRIPS STRIP

$6 \mathrm{~mm}$

238

$4.4 \mathrm{~mm}$

186

Total number non-bend strips $=198$ per sector.

Total number of bend strips $=\mathbf{2 , 2 2 2}$ per sector.

Total number of channels per sector $=\mathbf{2 , 4 2 0}$.

Total number of channels for GEM $=77,440$.

This is about 2.4 times the number of channels in the barrel.

The trigger logic for each bend plane set of 3 chambers (three $29^{\circ}$ chambers and three $16^{\circ}$ chambers) is identical to the barrel bend plane trigger logic. The same is true for the non-bend plane. Since there are two separate sets of chambers in the end cap, there will be two 64 bit data buses to carry the information to the second level trigger. The accidental rate and dead time calculation, however, are different. These calculations are discussed in Appendix B.

The layout and wiring of each endcap RPC chamber will be similar to the layout and wiring of the RPC chamber in the barrel. 


\section{Electronics}

The electronics for the trigger has to have the following features:

- Four momentum ranges - each range programmable

- Relative positions of each RPC plane programmable.

- Correction for interaction point programmable.

In addition the electronics must determine the centroid of the hit cluster - especially important in the endcaps. The beam crossing time must be determined, the strips which participated in the trigger must be isolated and recorded at Level 2, and multiple triggers - with their strip numbers are stored in memory for access at Level 2. Fig. 7.0.8 is a schematic of the electronics.

\section{SUMMARY}

The RPC trigger scheme has the following properties:

\section{Barrel}

1. Number of Channels

Non-bend plane

Bend plane

TOTAL

$\begin{array}{lll}498 \times 32 & = & 15,936 \\ 1659 \times 32 & = & 53,088 \\ 69,024\end{array}$

Strip Widths

Non-bend plane

SL1

SL2

$3.9 \mathrm{~cm}$

SL3

$6.5 \mathrm{~cm}$

Bend plane

$8.9 \mathrm{~cm}$

SL1

$1.3 \mathrm{~cm}$

SL2

$1.3 \mathrm{~cm}$

SL3

$1.3 \mathrm{~cm}$

\section{Strip Length}

Non-bend plane

SL1

SL2

SL3

Bend plane

SL1

SL2

SL3

$148.5 \mathrm{~cm}$

$239.0 \mathrm{~cm}$

$329.6 \mathrm{~cm}$

$631.9 \mathrm{~cm}$

$1,042.6 \mathrm{~cm}$

$1,475.0 \mathrm{~cm}$

Trigger acquisition time

100 nanoseconds 
Beam crossing error

First level trigger

11.7 nanoseconds

Second level trigger

Accidental rate at $\mathrm{L}=10^{34}$

5.0 nanoseconds

(transverse momentum $>50 \mathrm{GeV} / \mathrm{c}$

Dead time at a neutron flux of $10^{5}$ neutrons

$29 \quad$ Hertz

per square centimeters per second

(transverse momentum $>50 \mathrm{GeV} / \mathrm{c}$

Signal dead time gate $\quad=100$ nanoseconds $\quad 6 \%$

Signal dead time due to possible high rate inefficiency $16 \%$

End Cap

Detailed strip information is in Table 1.

Total number of end cap channels

Trigger acquisition time

77,440

Beam crossing error

First level trigger

100 nanoseconds

Second level trigger

11.7 nanoseconds

5 nanoseconds

All accidental rates and dead times are less than the barrel by more than an order of magnitude except if we assume each RPC chamber is only $90 \%$ efficient due to the high rate. In that case, the dead times in the barrel and end caps are the same. 


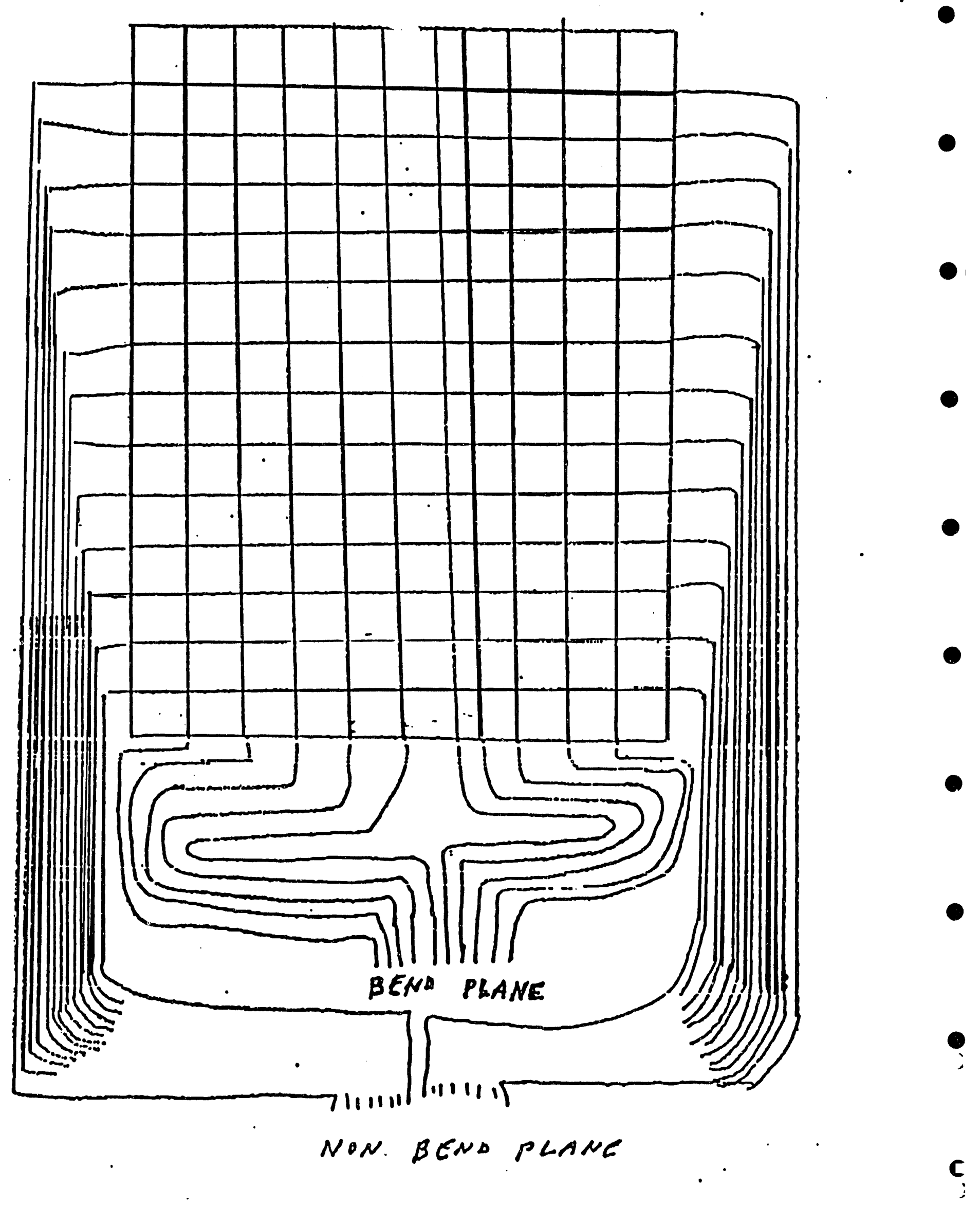

Fig. 7.0.1. 
ต
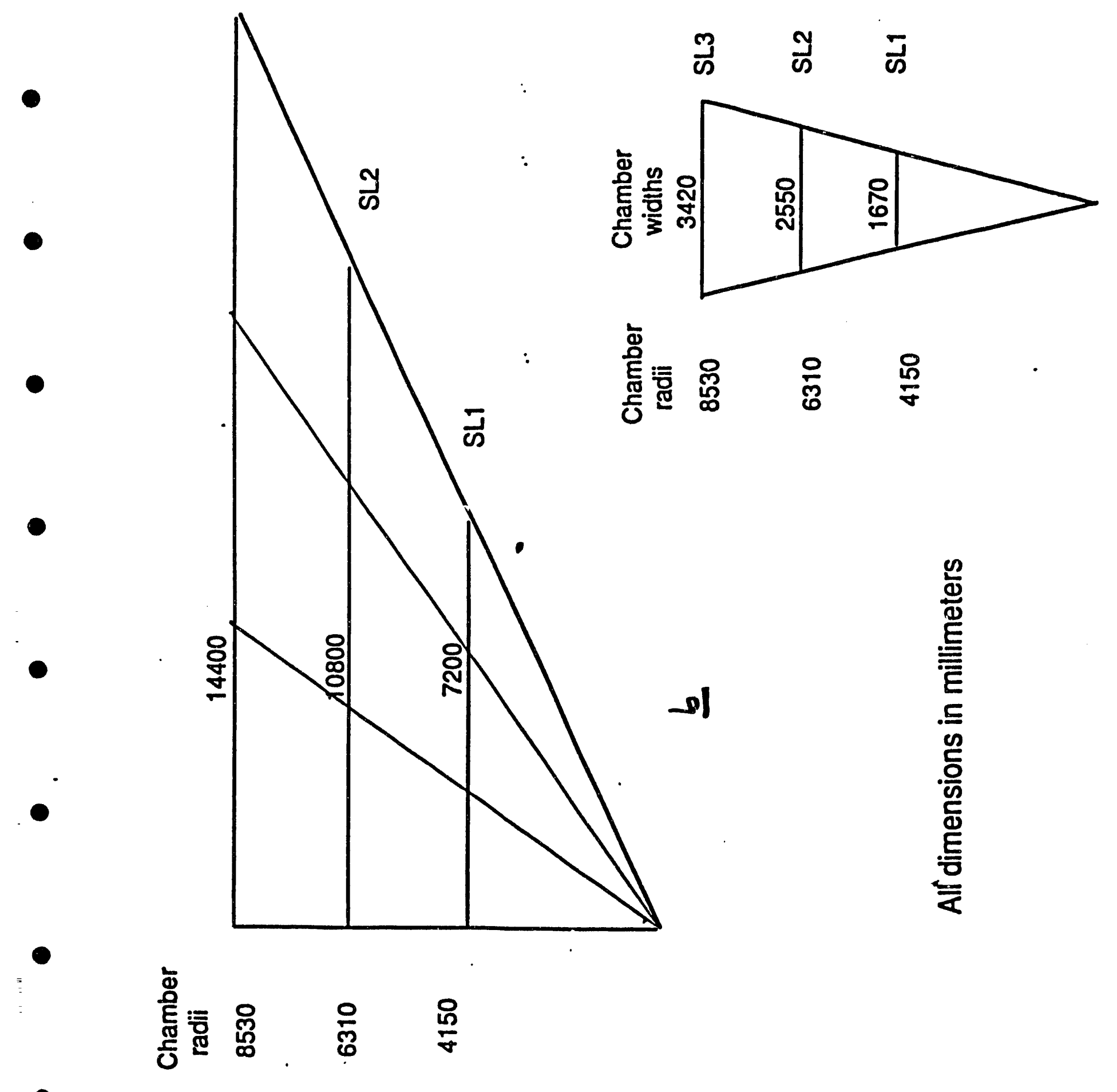

$\because$
$\dot{0}$
$\dot{0}$
$\dot{0}$ 


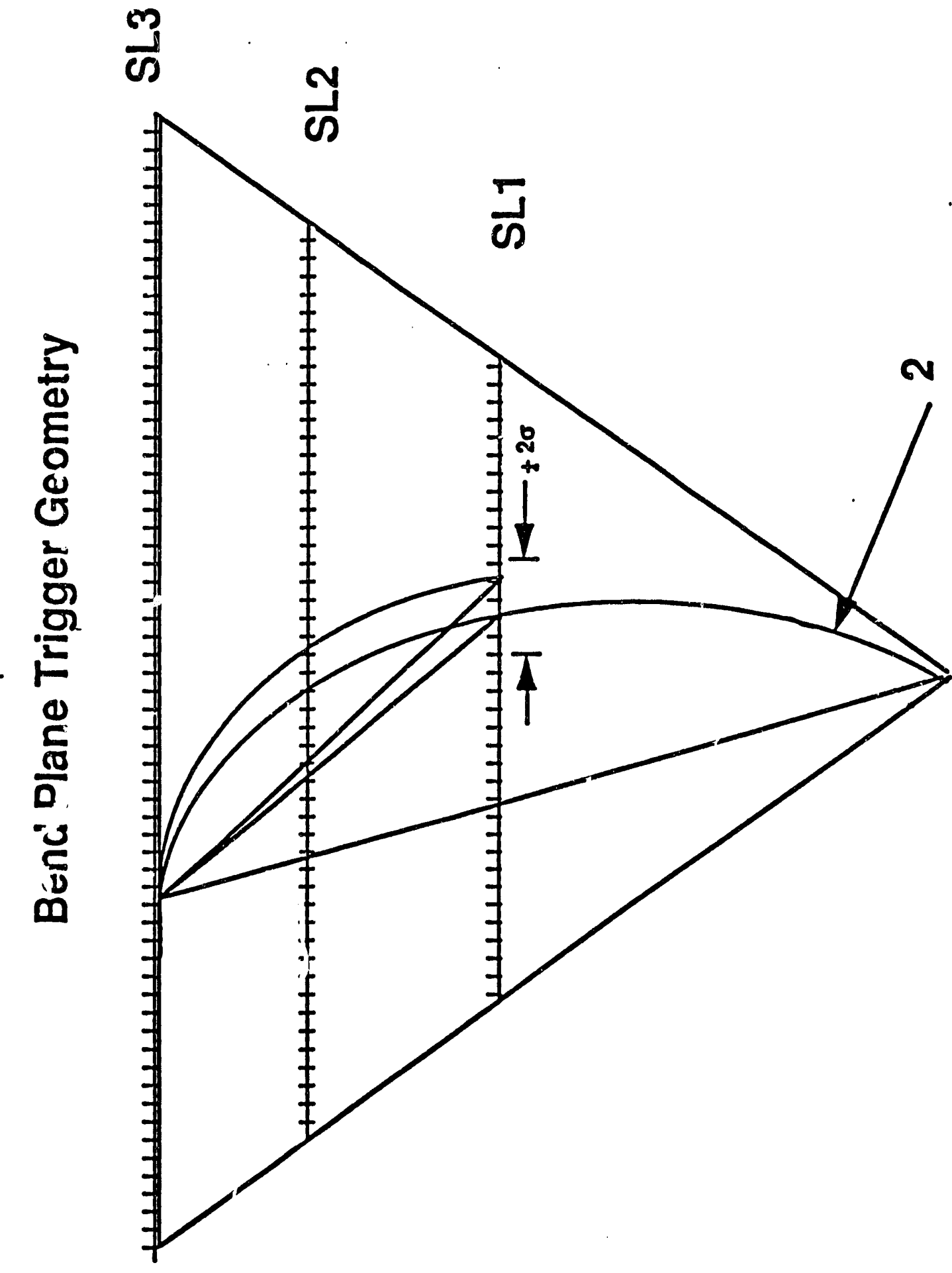

ro 


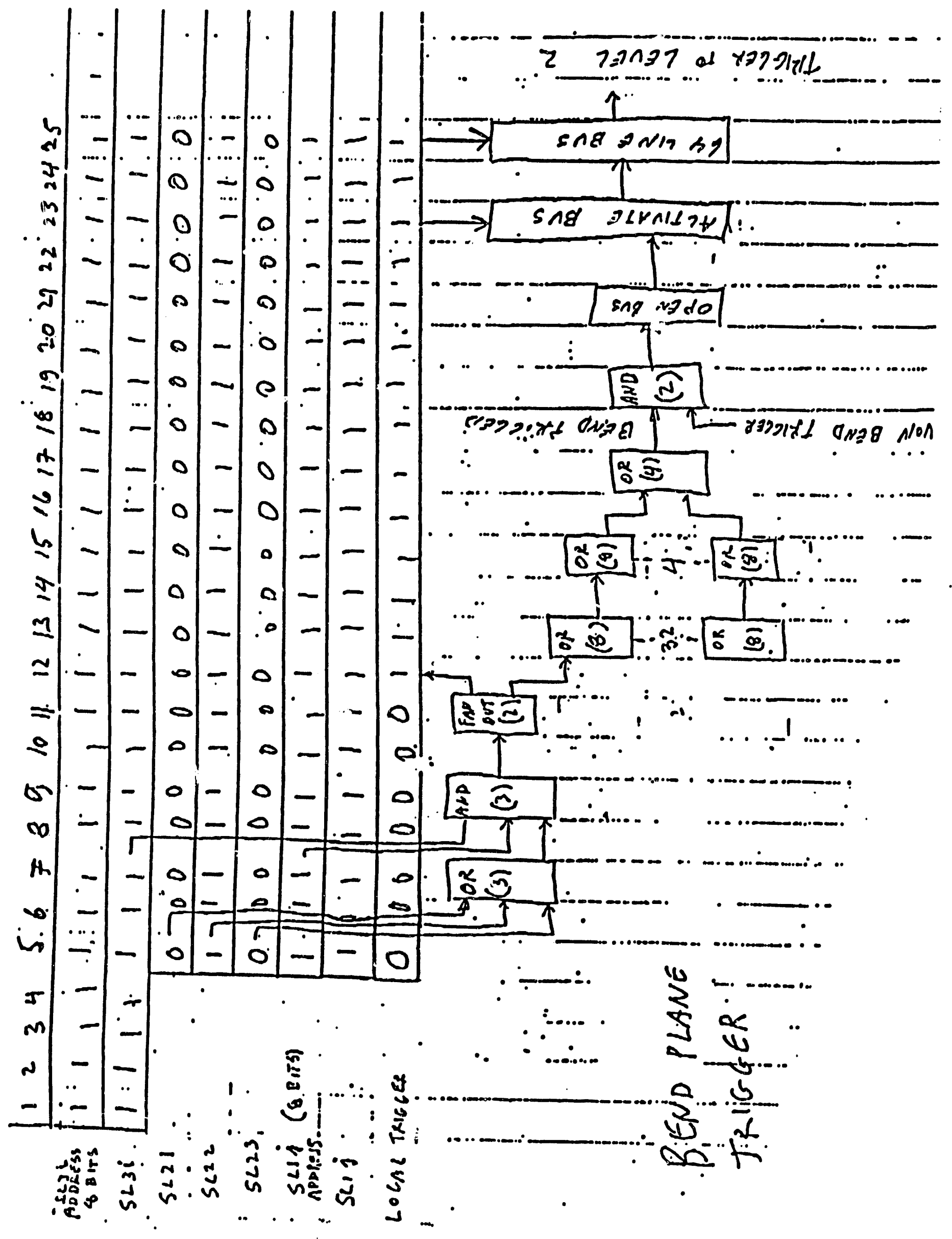




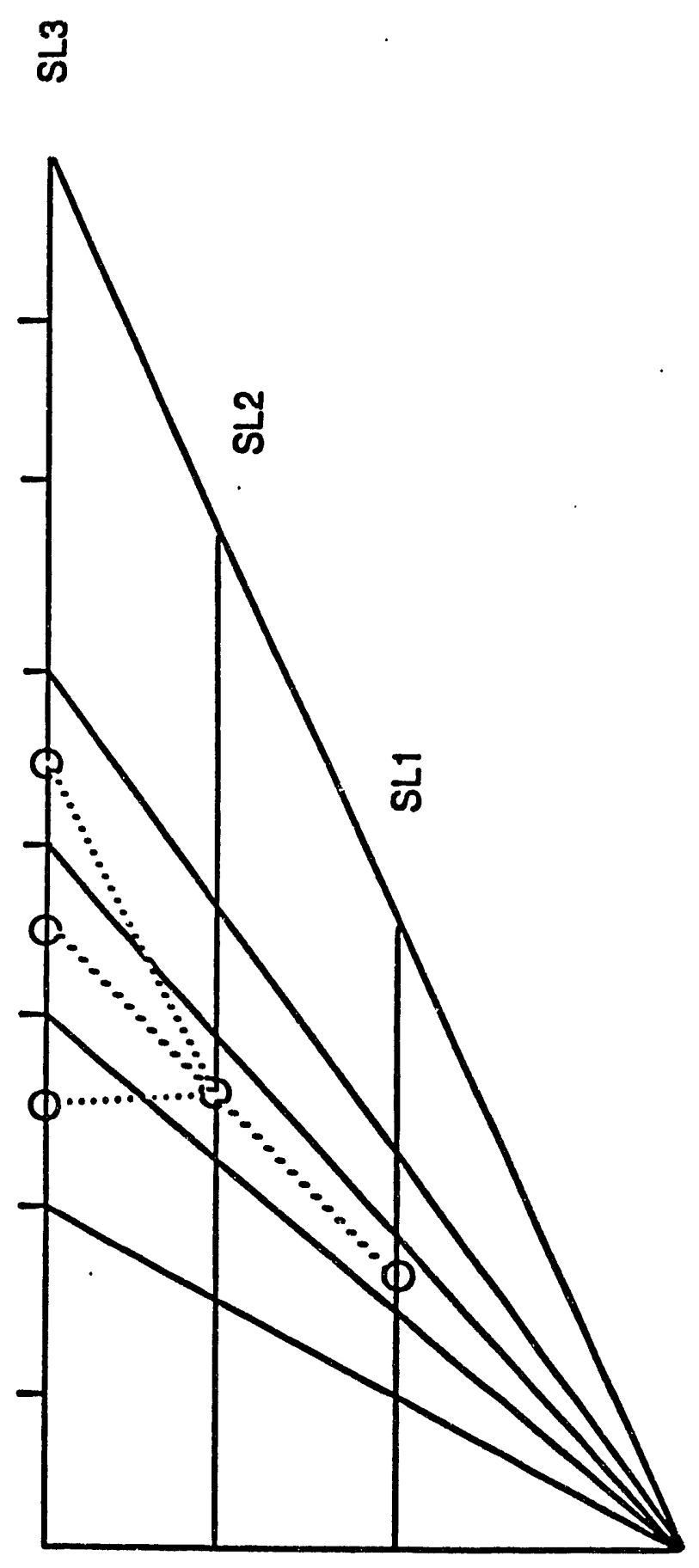

140 


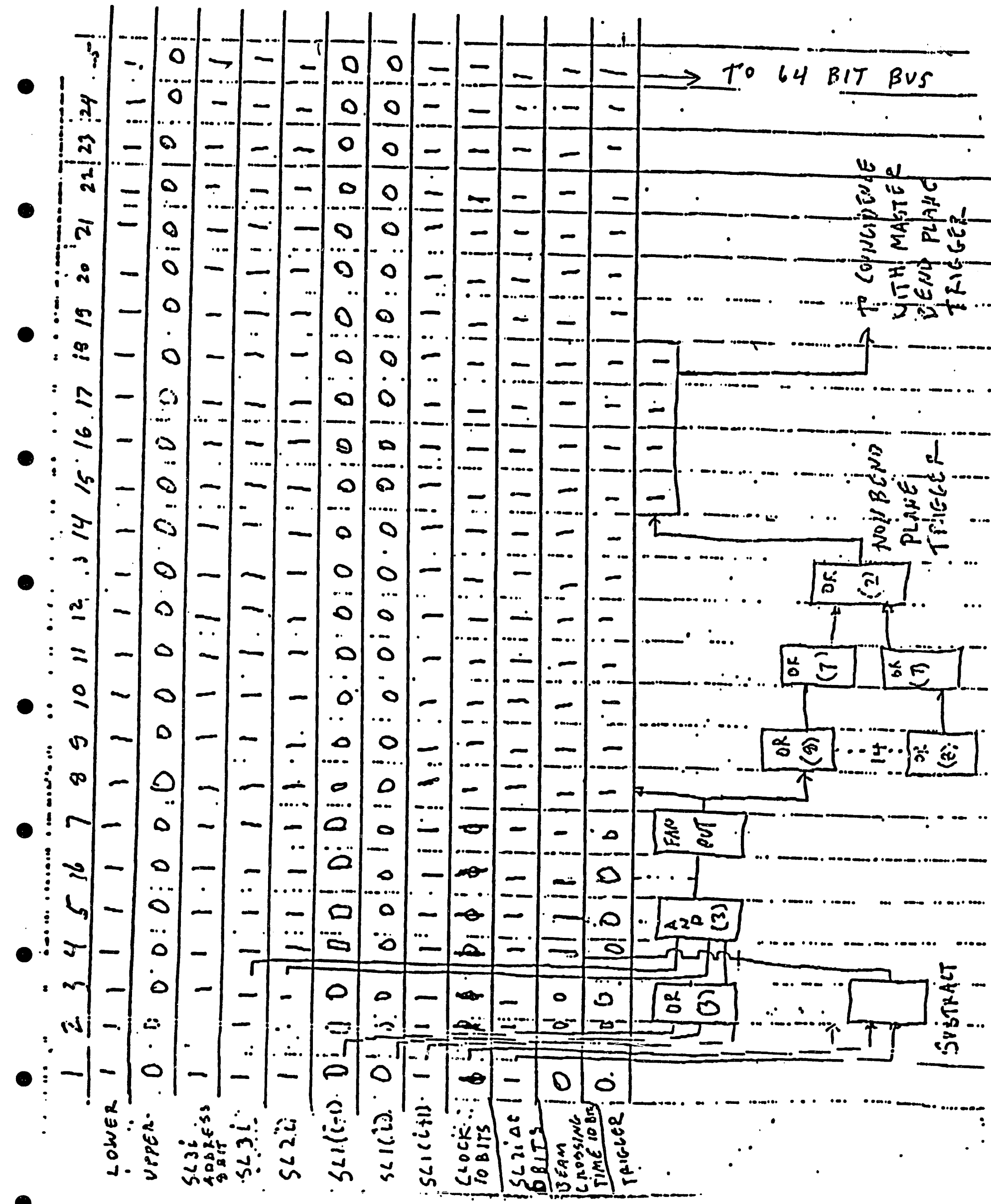

0
$\dot{0}$
$\dot{0}$
$\dot{0}$
$\dot{0}=1$ 


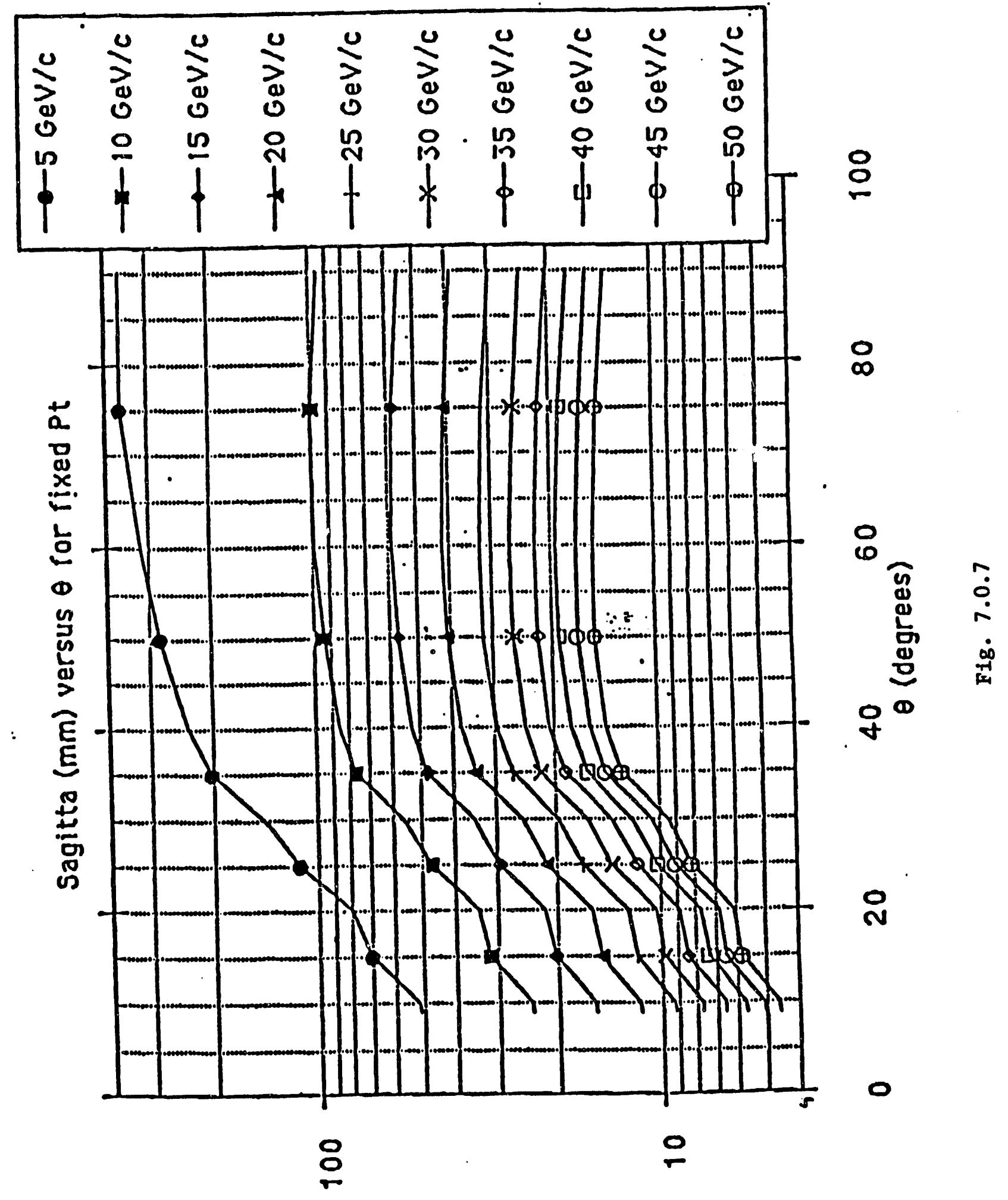




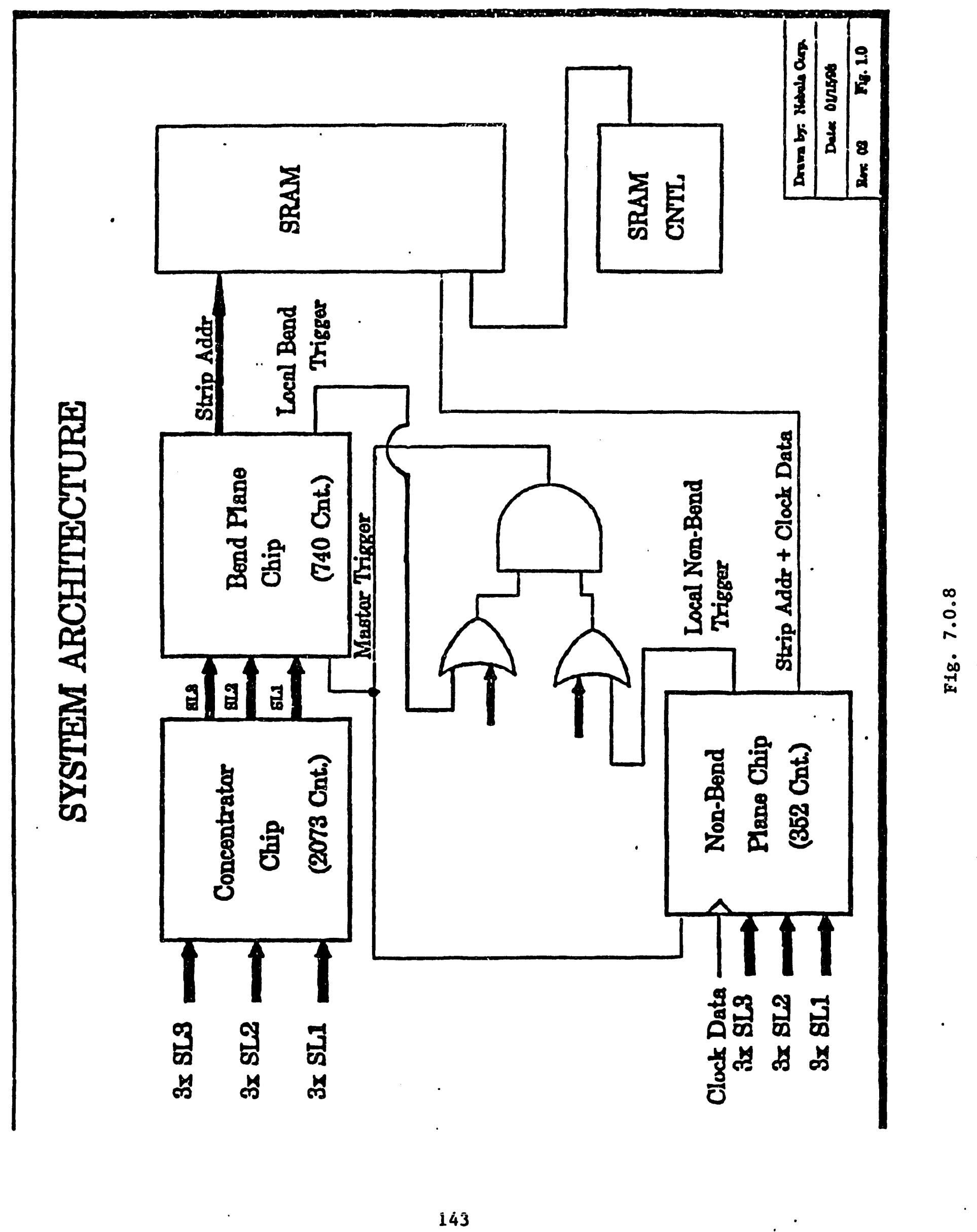




\section{(7.1) RDT Data Acquisition}

It is proposed that the readout of the RDT chamber data of a Level-1 trigger be track driven; only tubes which are part of a local track segment (3-of-4 tubes) are readout. Each super-layer contains two packages of either 4 or 6-tube layers that are grouped into sections with at most 24 tubes per section as shown in the figure below (i.e. 6 tubes/layer, the package with $6 x$-layers will have two such groups with two layers in common).

Superlayer Track Sections

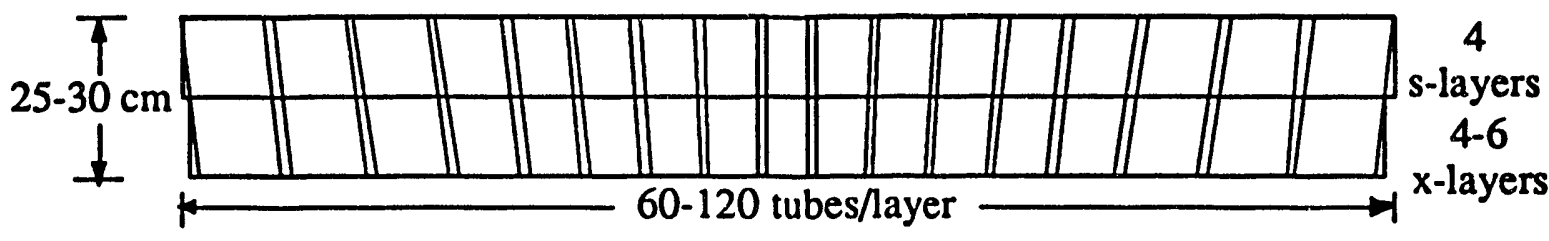

There is one tube/layer overlap between sections to allow a track to cross between sections or for stereo angle effects. The fast pattern logic shown below determines if a track segment exists in a section.

Track-Segment Pattern Logic

(Max. Effective Rates)

(single-double buffered timing)

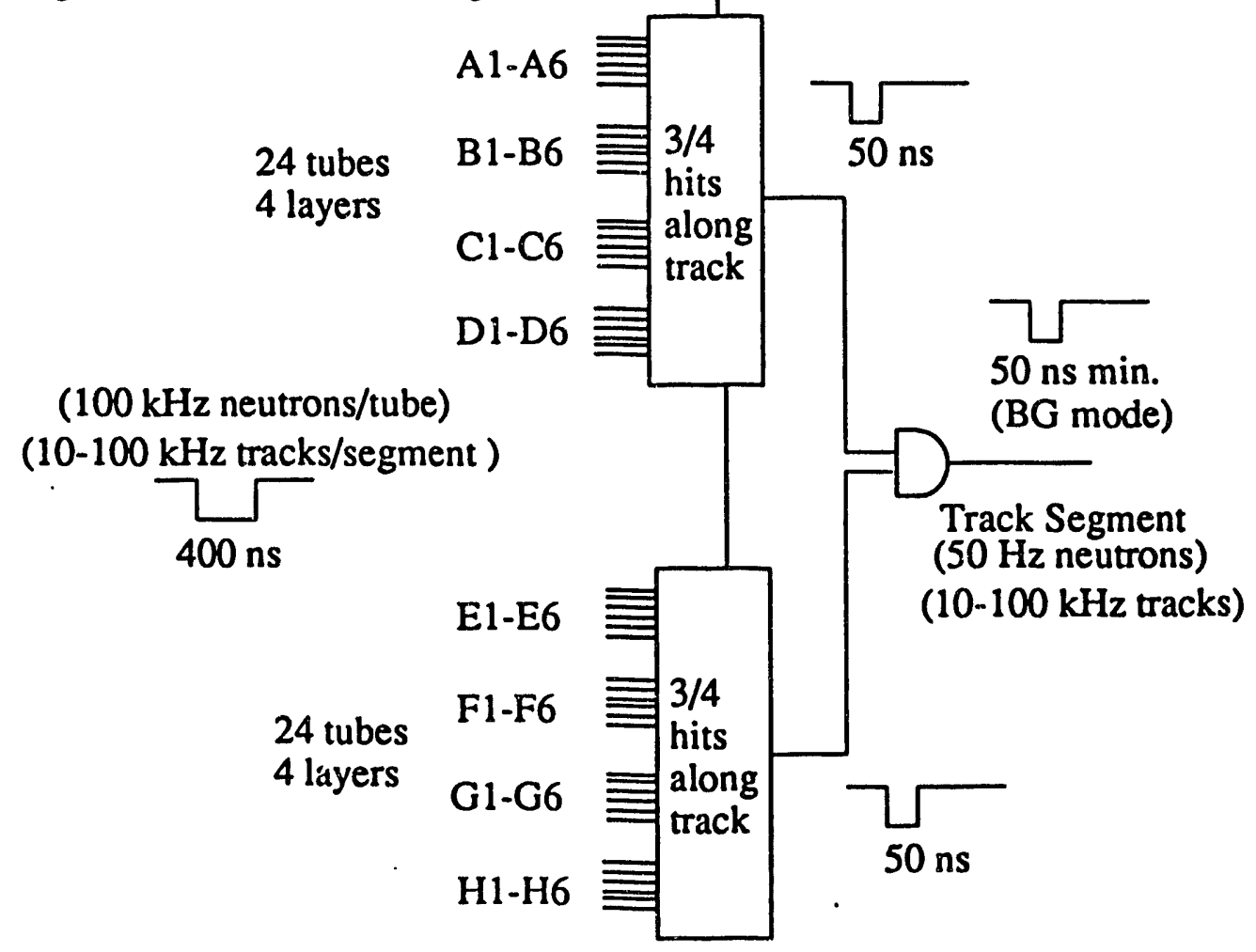


The 6 tubes/layer in each section are grouped into two 16M-bit memories (224). A 3-of- 4 requirement is coded into the memories. A 1-bit in the memory signifies a legal track pattern is formed by the 24 tubes (address lines of the memory) in a section. If the upper(A-D) and lower(E-H) sections when strobed (every $50 \mathrm{~ns}$ ) both satisfy the 3-of-4 requirement a track signal is formed.

This logic will reduce the neutron rate to well below the true track rate. This means that only true tracks will be readout on a Level-1 trigger and the large number of random neutron hits will be suppressed. The dead time of this logic is not serious if the subsequent timing is double buffered. In the event that two tracks cross a section within a drift-time the width of the Track Segment output will signify when it is safe to stop the second buffer. In this manner two tracks with any relative timing within the 2 msec Level- 1 trigger delay can be recorded for readout. Dead-time can be incurred only if there are three tracks within a section within the Level-1 trigger delay.

The time of the most recent hit is latched via a common stop signal generated soon after a Track Segment signal is transmitted from the pattern logic. The stop time is referenced to a fixed phase of the accelerator clock to better than 1 nsec.

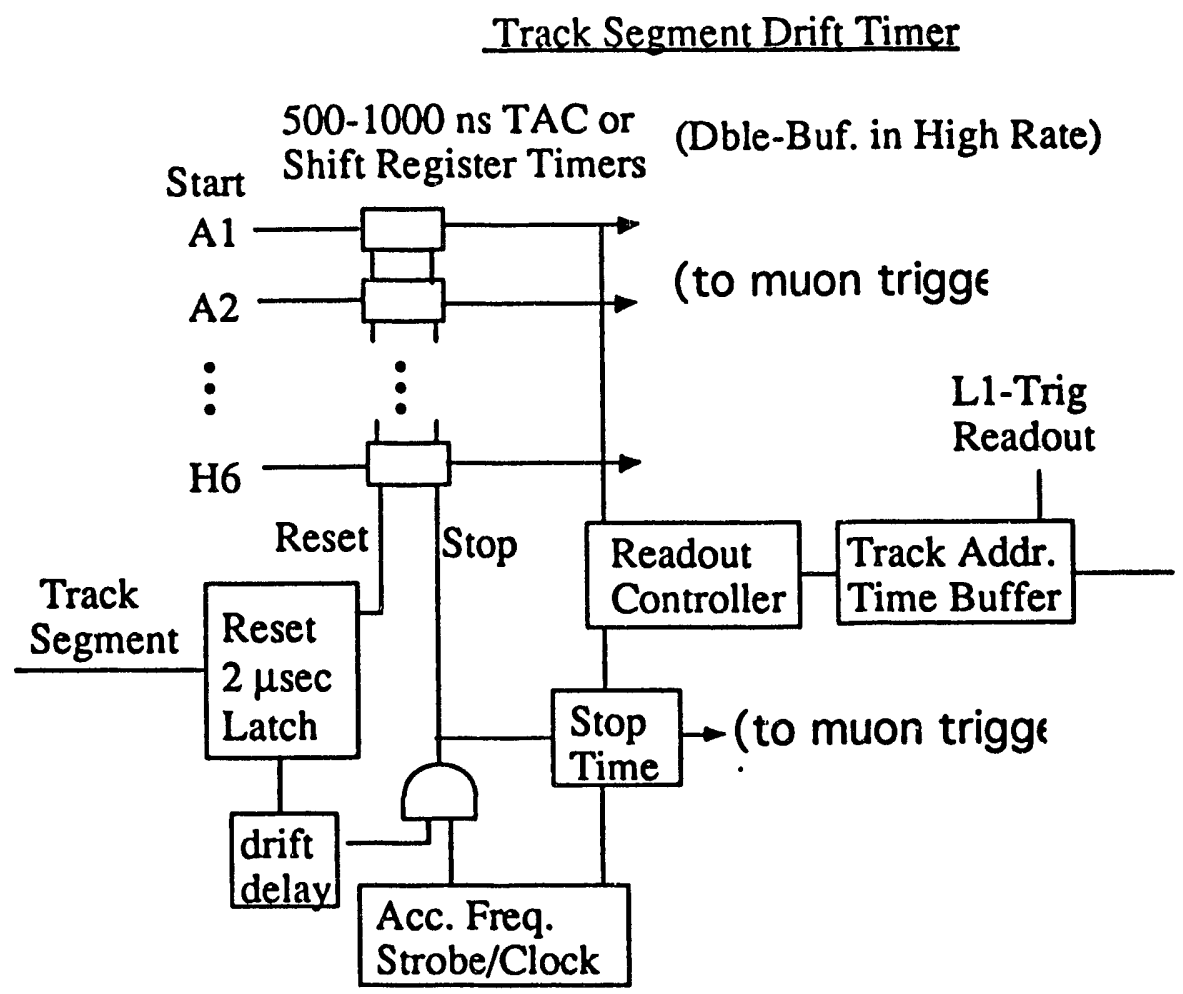

The timing element will be a shift-register or a time-to-amplitude converter. In either case the common stop time from an absolute bucket scaler is also latched by the stop signal. In less than $1.5 \mu \mathrm{sec}$ while a Level-1 trigger from any source is being formed 
the non-zero times and tube addresses are loaded into a buffer. If a level-1 trigger does not arrive by $2.0 \mu \mathrm{sec}$ the timing elements are reset. The timing elements will be double buffered in regions where the track-rate is high enough to cause significant dead-time. The individual times and the stop time are also made available to a RDT stand-alone trigger system.

The common stop time is relevant only for the track in this section. Each track segment will have its own stop time recorded. In the chambers at the smallest angles to the beam the expected track rate in a section may exceed $10 \mathrm{kHz}$ ( $2 \%$ dead time for the Timers). For these chambers and any other region where the rate exceeds 10 $\mathrm{kHz} /$ section the Timer circuitry will be double buffered to allow two tracks within $2 \mu \mathrm{sec}$ to be latched. Since a small loss of trigger efficiency can be tolerated at small angles it does not seem necessary to duplicate the trigger logic for the second track.

In the RDT baseline there are 26 tubes which will lie along a track in either the barrel or the endcap. The time of these signals relative to the stop time is known off-line to better than 1 nsec and can be determined on-board the chambers quickly to better than \pm 5 nsec. 


\section{(7.2) PT-Trigger Based on RDT}

A number of viable $\Upsilon_{0}$ and $P_{T}$-triggering schemes have been discussed within the RDT group. The RDT system can be auto-triggered, as in the scheme of A. Korytov and B. Barabash (see Appendix E). Another scheme is to use the scintillators of the tail catcher of the calorimeter or a standalone PWC system (as a substitute to the $\mathrm{RPCs}$ ) to generate a T0 signal needed for the drift trigger.

A description of one of these auto-triggering schemes is presented here. Track segments in the three superlayers consistent with the trigger $\mathrm{P}_{\mathrm{T}}$ determine the slope of the track $b_{0}$ at each superlayer to better than $10 \mathrm{mrad}$. At most one hit tube in each layer is then identified with the track from the observed hit-pattern. Shown below are a set of hit patterns and typical track limits defined by the $b_{0}$ constraint. The $b_{0}$ constraint can also be used to resolve the small number of ambiguous cases. The object here is to identify the direction of the drift in each tube which is needed to determine $t_{0}$. 
4 Tube Patterns and Range of Track Solutions

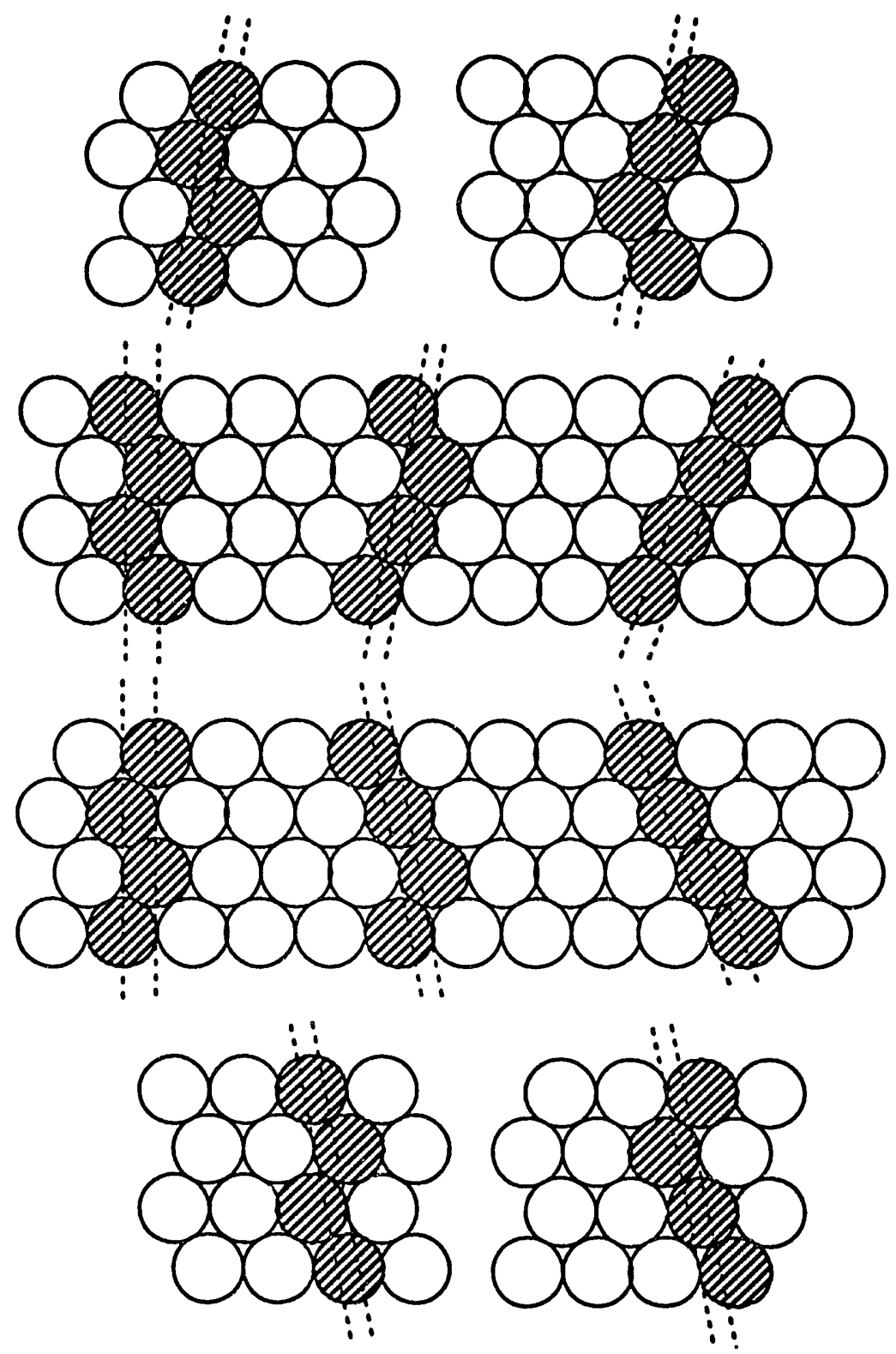

Of course if to is determined by another device, such as a PWC, the a measurement of the transverse momentum can be made from these patterns alone.

An improved superlayer crossing point $\alpha$ and a preliminary interaction time to can then be calculated in each package using a constant drift velocity. The example shown below has a 1-r-1-r pattern of drifts, however, only the signs change in algorithm for other patterns. For less than 4 good hits the averaging is done only over the good hits. 
Let: $Z_{i}=(+3,+1,-1,-3) \Delta Z, \Delta Z=1 \mathrm{~cm}, v i= \pm v_{s}$ and $c_{i}=n_{i} t_{i}+b_{0} Z_{i}$. Then superlayer crossing point and the estimator of the interaction time is given by:

$$
\begin{aligned}
& \alpha=\langle c\rangle=\frac{v\left(-t_{1}+t_{2}-t_{3}+t_{4}\right)}{4} \text { for } v i=-v,+v,-v,+v \\
& \tau_{0}=\frac{(v c)}{\left\langle v^{2}\right\rangle}=\frac{\left(t_{1}+t_{2}+t_{3}+t_{4}\right)}{4}+\frac{b_{0} \Delta Z}{v}
\end{aligned}
$$

Once $t_{0}$ and $\alpha$ are determined the correct velocities using the true time-to-distance relationship can be used to obtain the precise interaction time $t_{0}$, crossing point $\alpha$, and slope b. Each of these computations is performed via table lookups rather than a true calculation. With 26 tubes the identification of the true bucket crossing for the track and the momentum seem assured. 


\section{(8.0) System Performance}

In this section the resolutions of the trigger, momentum reconstruction, and angle reconstruction are described. All have been calculated from the parameters of the system given in Section (3) above.

\section{(8.1) Trigger Performance:}

\section{(8.1.1) Momentum Resolution:}

The trigger algorithm is based on a measurement of the sagitta in the barrel and the projected sagitta (within a small-angle cosine correction equal to the true sagitta). This method has been adapted over the $\Delta \phi$ method since it has superior resolution which will be crucial in limiting the Level 1 trigger rate at high luminosity. A typical set of parameters for this trigger scheme is shown in the table below:

Table 8.1

RPC Trigger Parameters for $\mathrm{Pt}=10 \mathrm{GeV} / \mathrm{c}$

\begin{tabular}{|c|c|c|c|c|}
\hline Region number & \multicolumn{2}{|c|}{ angle limits element size } & $\eta$ limits & sagitta cuts \\
\hline 1 & 57 to 90 & $1.3 \mathrm{~cm}$ & 0 to 0.6 & $9.0 \mathrm{~cm}$ \\
\hline 2 & 39 to 57 & $1.3 \mathrm{~cm}$ & 0.6 to 1.04 & $9.0 \mathrm{~cm}$ \\
\hline 3 & 29 to 39 & $1.3 \mathrm{~cm}$ & 1.04 to 1.35 & $8.0 \mathrm{~cm}$ \\
\hline 4 & 22.5 to 29 & $0.6 \mathrm{~cm}$ & 1.35 to 1.61 & $7.5 \mathrm{~cm}$ \\
\hline 5 & 16 to 22.5 & $0.6 \mathrm{~cm}$ & 1.61 to 1.96 & $5.0 \mathrm{~cm}$ \\
\hline 6 & 9.75 to 16 & $0.44 \mathrm{~cm}$ & 1.96 to 2.46 & $2.7 \mathrm{~cm}$ \\
\hline
\end{tabular}

The trigger threshold performance was simulated by computing the (projected) sagitta as would be measured with the finite trigger elements of system. Multiple scattering and energy loss with fluctuations were included in the calculation. But note that the sagitta method is not sensitive to the multiple scattering in the calorimeter. The muon was allowed to have the full $\phi$ range of a given chamber (11.50 for the barrel and $22.5^{\circ}$ for the endcaps). The results are shown in Fig. 8.1.1(a), 8.1.1(b), 8.1.1(c) for (primordial) $\mathrm{Pt}=10,25,50 \mathrm{GeV} / \mathrm{c}$, respectively. Note that the projected sagitta in the endcaps leads to a small additional smearing of the trigger threshold. However the thresholds are still quite good even with this effect. To reduce (but is not needed) the $\phi$ smearing arising from the rectangular chambers in the endcaps the following strategy may be adapted. If a track penetrates the overlap region, the coincidence can be detected and trigger cut be made on the larger of the 

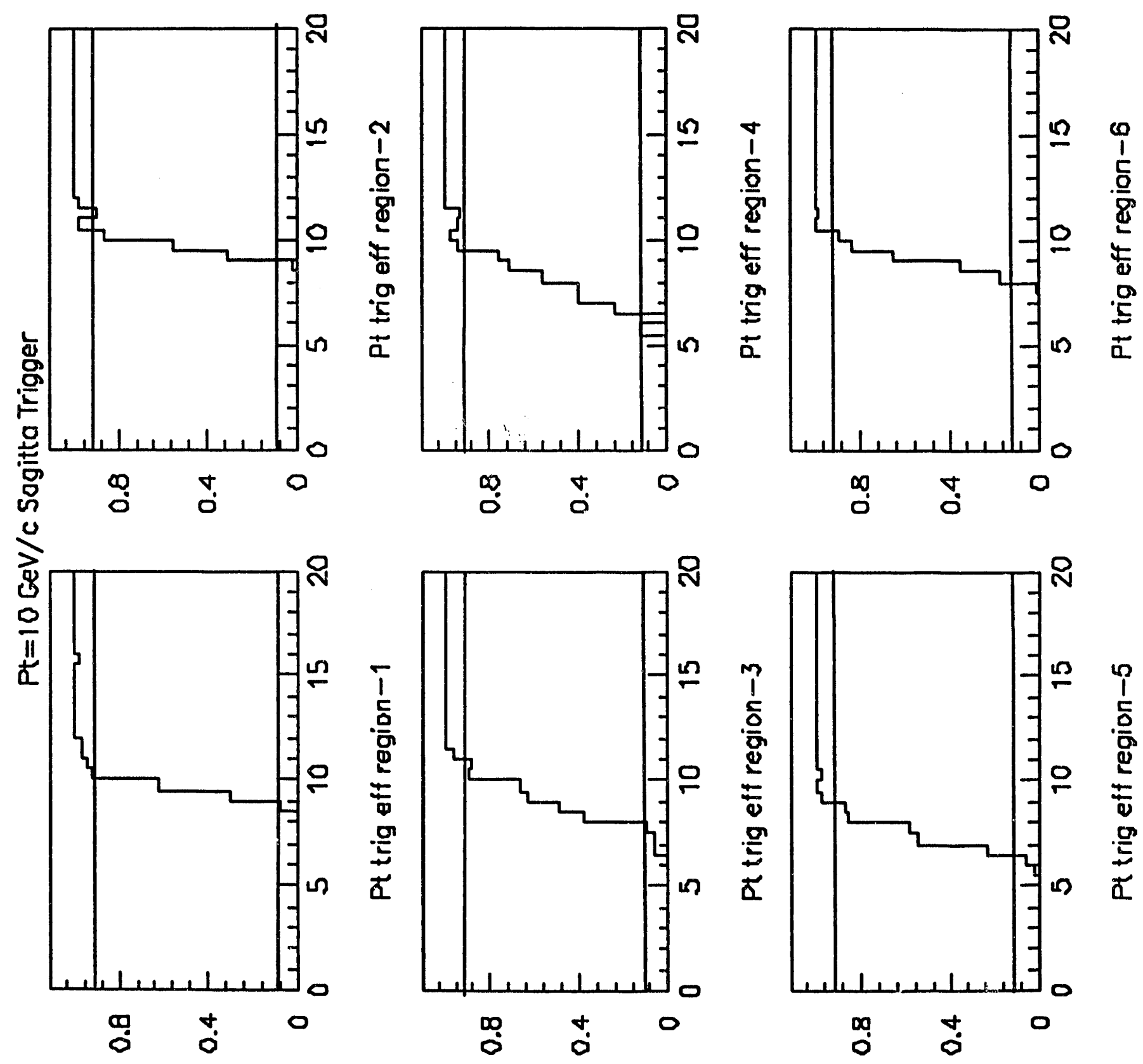


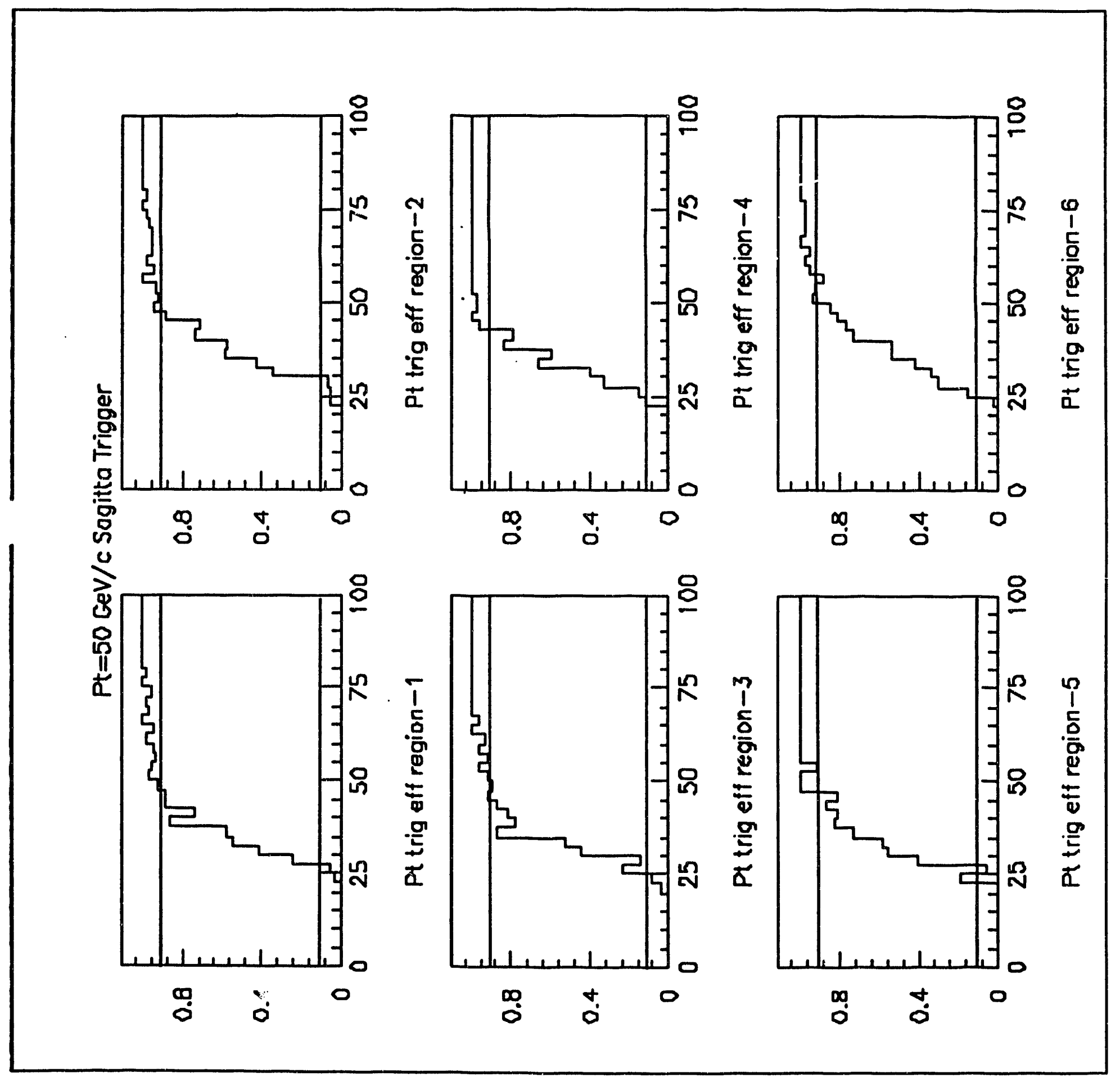


two projected sagitta values. Where the $\phi$ smearing is large in one chamber it is small in the other.

In contrast to the sagitta method adapted here, the $\Delta \phi$ method with radial strips in the endcaps as applied between superlayers 2 and 3 as presently proposed has considerably less resolution since it is dominated by multiple scattering in the calorimeter. A typical threshold curve computed by this scheme is shown in Fig. 8.1.2. This trigger method will not achieve the needed low $\mathrm{P}_{\mathrm{T}}$-rejection at high luminosities.

\section{(8.1.2) Rate Performance:}

The difficulty with the present RPC trigger arises from the rate limitation (97\% efficient for rates $<50 \mathrm{~Hz} / \mathrm{cm}^{2}$ ) of the present version of this technology. Neutron fluences of order $10^{4} / \mathrm{cm}^{2} \mathrm{sec}$ are expected in the barrel region at $\mathrm{L}=10^{33} / \mathrm{cm}^{2} \mathrm{sec}$ and roughly 10 times higher in the endcaps. At these rates and with the measured sensitivity of the RPC to neutrons of $0.5 \%$, we find the neutron rate "burden" in the barrel to be $50 \mathrm{~Hz} / \mathrm{cm}^{2}$ and $500 \mathrm{~Hz} / \mathrm{cm}^{2}$ in the endcaps. The barrel environment is then just at the rate limit of the Italian RPC and the endcaps severely beyond. The charged particle flux in the very forward angle region is calculated to be $<50$ $\mathrm{Hz} / \mathrm{cm}^{2}$ at $\mathrm{L}=10^{33}$. Hence the $\mathrm{RPC}$ in its present design is marginal at $\mathrm{L}=1033 \mathrm{in}$ the barrel region and not viable in the endcaps.

The neutron fluence calculations are very uncertain and there is some reason to believe that shielding will mitigate the problem. Furthermore there have been promising results in finding new materials for the RPC resistive layer which would withstand much higher rates (Peskov - FNAL private communication). Major uncertainties remain however and it is for these reasons that we propose a PWC trigger system to replace the RPCs. Such a device could be an inexpensive version of the present CSC design where roughly $80 \%$ of the costs go into high precision elements. At the present pricing of chambers a 4-3-3 trigger system would cost roughly $\$ 8 \mathrm{M}$. See the Cost Appendix C.

The random hit occupancy for a typical drift tube (where two tubes are tied together at one end) is about $5 \%$ for a neutron fluence of $10^{4} \mathrm{~Hz} / \mathrm{cm}^{2}$. Roughly the same numbers pertain to a CSC system with an integration time of $500 \mathrm{~ns}$.

\section{(8.2) Momentum Reconstruction:}

The momentum resolution of the system is determined by the number of chamber layers, the layer resolution, the multiple scattering in the middle superlayer, and the fluctuations of the energy loss in the calorimeter. The event reconstruction of two important processes drive the specification of the system. 


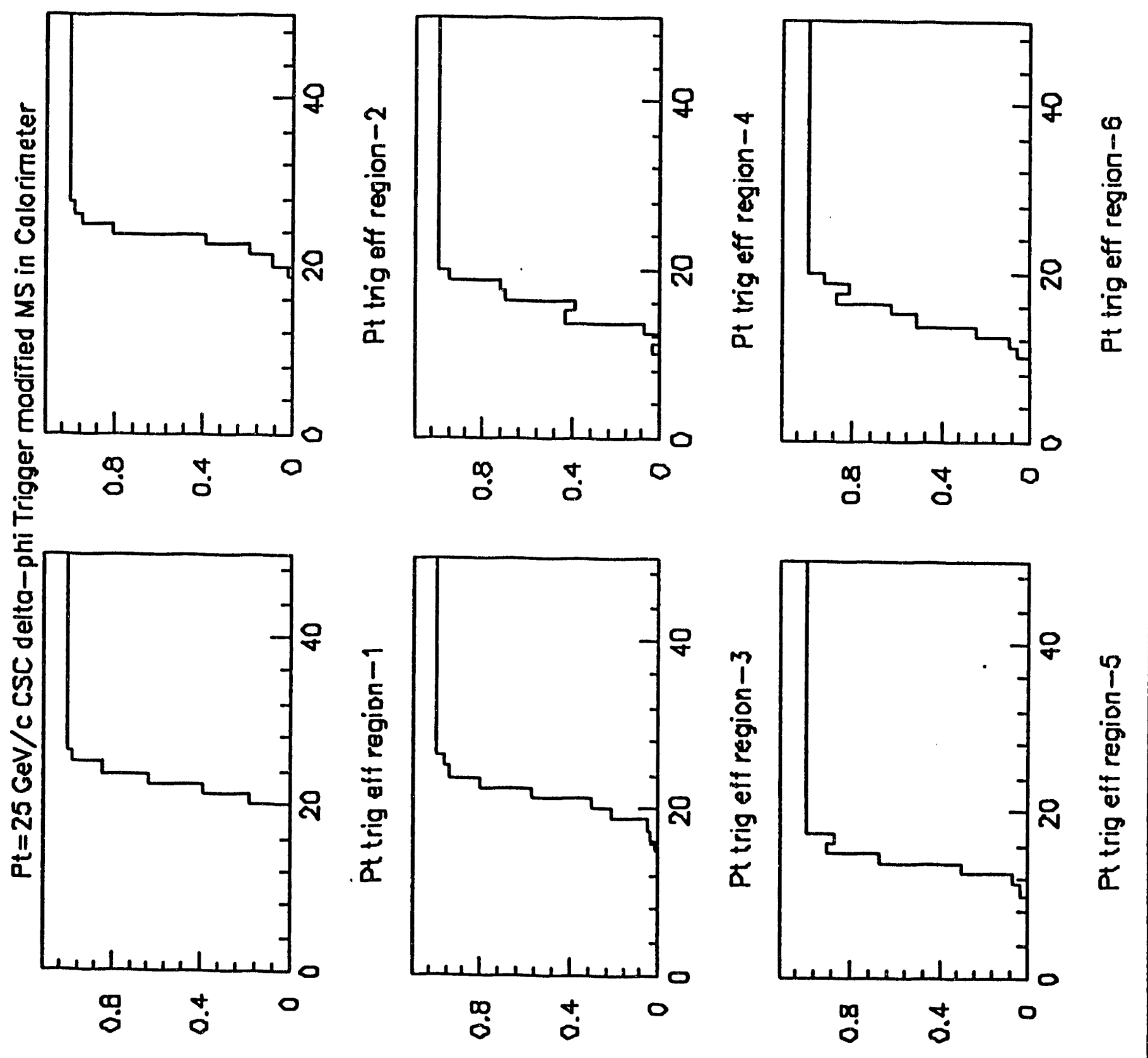


- Low mass Higgs boson $\mathrm{MH}=130 \mathrm{GeV} / \mathrm{c}^{2}$ where the transverse momenta of the decay muons range from $10 \mathrm{GeV} / \mathrm{c}$ to $100 \mathrm{GeV} / \mathrm{c}$.

- High mass $Z^{\prime}$ boson $\mathrm{MZ}^{\prime}=1 \mathrm{TeV} / \mathrm{c}^{2}$, where the analysis of muons in 0.5 to $1 \mathrm{TeV} / \mathrm{c}$ range is needed.

In the low momentum region, the fluctuations of the energy loss of the muons traversing the calorimeter is the dominate factor. The energy loss of the muon in the calorimeter can in principle be reconstructed, but would require isolating the muon energy loss ( $\Delta \mathrm{E} \mu=5$ to $10 \mathrm{GeV}$ ) from a noisy background of much greater energy deposition and resolving the $\Delta \mathrm{E} \mu$ with an error of $20 \% / \sqrt{ }(\Delta \mathrm{E} \mu)$. Less important is the multiple scattering of the muon in the middle superlayer.

Key to the reconstruction of high energy momenta is to have enough chamber planes with clean hits. This is why we have exploited the economic chamber construction of this system to allow for high redundancy. We believe that this is an important design parameter of the system. To illustrate this point Table 8.2 give the fraction of clean events observed for a $1 \mathrm{TeV} / \mathrm{c}$ muon traversing the detector at 90 degrees. These parameters have been used in a fast simulation of the high energy system performance. We have assumed that the single layer resolution of the RDT system is $100 \mu \mathrm{m}$ even though full-scale prototypes have demonstrated a resolution as good as $60 \mu \mathrm{m}$.

\section{Table 8.2}

Ratio of good hits (golden hits) for $1 \mathrm{TeV} / \mathrm{c}$ Muons at 90 degrees

\begin{tabular}{|c|c|c|c|}
\hline hits required & Inner & Middle & Outer \\
\hline $3 / 4$ & $0.873(0.825)$ & $0.918(0.888)$ & $0.943(0.910)$ \\
\hline $4 / 4$ & $0.743(0.703)$ & $0.810(0.785)$ & $0.835(0.813)$ \\
\hline $4 / 6$ & $0.893(0.830)$ & $0.928(0.900)$ & $0.958(0.928)$ \\
\hline $5 / 6$ & $0.810(0.760)$ & $0.888(0.855)$ & $0.893(0.863)$ \\
\hline $6 / 6$ & $0.675(0.638)$ & $0.758(0.730)$ & $0.745(0.728)$ \\
\hline $4 / 8$ & $0.933(0.880)$ & $0.910(0.925)$ & $0.968(0.948)$ \\
\hline $6 / 8$ & $0.870(0.795)$ & $0.858(0.880)$ & $0.940(0.913)$ \\
\hline $8 / 8$ & $0.630(0.605)$ & $0.700(0.683)$ & $0.710(0.700)$ \\
\hline
\end{tabular}

We note that at least 8 layers are required in the inner superlayer for at least 4 good hits $>90 \%$ time, and at least 6 layers are required in the outer superlayer for the same criterion. Our 8:10:8 chamber configuration meets this requirement. Both the small angle stereo planes and axial layers determine [within a very small effect of order $1 / \cos (4 \mathrm{mr})$ ] the same coordinate for the momentum reconstruction. In addition 
the U-V planes are paired to determine the $Y$ coordinate with a resolution indicated in Section 2.1 of this Baseline.

The momentum resolution in the $\mathrm{PT}=10$ to $100 \mathrm{GeV} / \mathrm{c}$ region is given in Fig. 8.2.1a. (The worse resolution sector of the endcaps was chosen for this calculation - see below.) Note that the resolution improves with increasing momentum in this region since the dominate effect is the un-reconstructed energy fluctuations in the calorimeter. The resolution is computed in the overlap region of the endcaps in Fig. 8.2.1b. There the chamber configuration is effectively 16-20-16 and the multiple scattering in the middle superlayer will be a larger effect. That we observe in the 25 to $50 \mathrm{GeV} / \mathrm{c}$ range where the resolution is degraded from $2.5 \%$ to $3 \%$.

The high energy performance, where the energy fluctuations in the energy loss in the calorimeter can be neglected, is shown in Fig. 8.2.2a and 8.2.2b for the two angle sectors of the endcaps. For this calculation we have assumed that all the chamber planes provide unspoiled hits. The resolution in the overlap region of the endcaps is somewhat better. In that region the number of planes in the middle superlayer is effectively 20 providing a better resolution, or equivalently there are two determinations of the momentum which can be treated as independent measurements, but with $2 x$ multiple scattering. The resolution in that region is indicated in Fig. 8.2.2c. Roughly 30\% of the single muons will traverse this region.

From the table above we note that the resolutions of Fig. 8.2 are optimistic and do not account for spurious hits associated with a high energy muon track. The get a measure of this effect the probabilities of the Table 8.2 were converted to a binomial distribution to provide an estimator (uncorrelated) of the number of good hits participating in the track reconstruction. These probabilities are illustrated in Fig. 8.2.3a,b for the barrel and endcaps, respectively. Putting these into the computation of the resolutions Fig. 8.2.3c indicates that the resolution degrades from only about $23.3 \%$ to $24 \%$ for $\eta=2.5$ for an 8-10-8 at $1 \mathrm{TeV} / \mathrm{c}$. A muon system with fewer planes degrades more rapidly, again illustrating the need for a large number of planes in the muon system.

The angle resolutions are shown in Figs. 8.2.4a and 8.2.4b. We have used the $Y$ resolution obtained by the small angle stereo to achieve the $\theta$ resolutions shown. The RPC also determines this angle and will be used as a redundant measurement. At low momentum the polar angle resolution is dominated by the multiple scattering in the calorimeter and at high momenta by the spatial resolution of the system. The $\phi$ resolution is dominated by the multiple scattering in the superlayers at low momentum and by the spatial resolution of the precision coordinate measurement at high momentum.

In summary the RDT system proposed has good performance and a high redundancy of chamber planes - needed for a good muon system at the SSCL. 


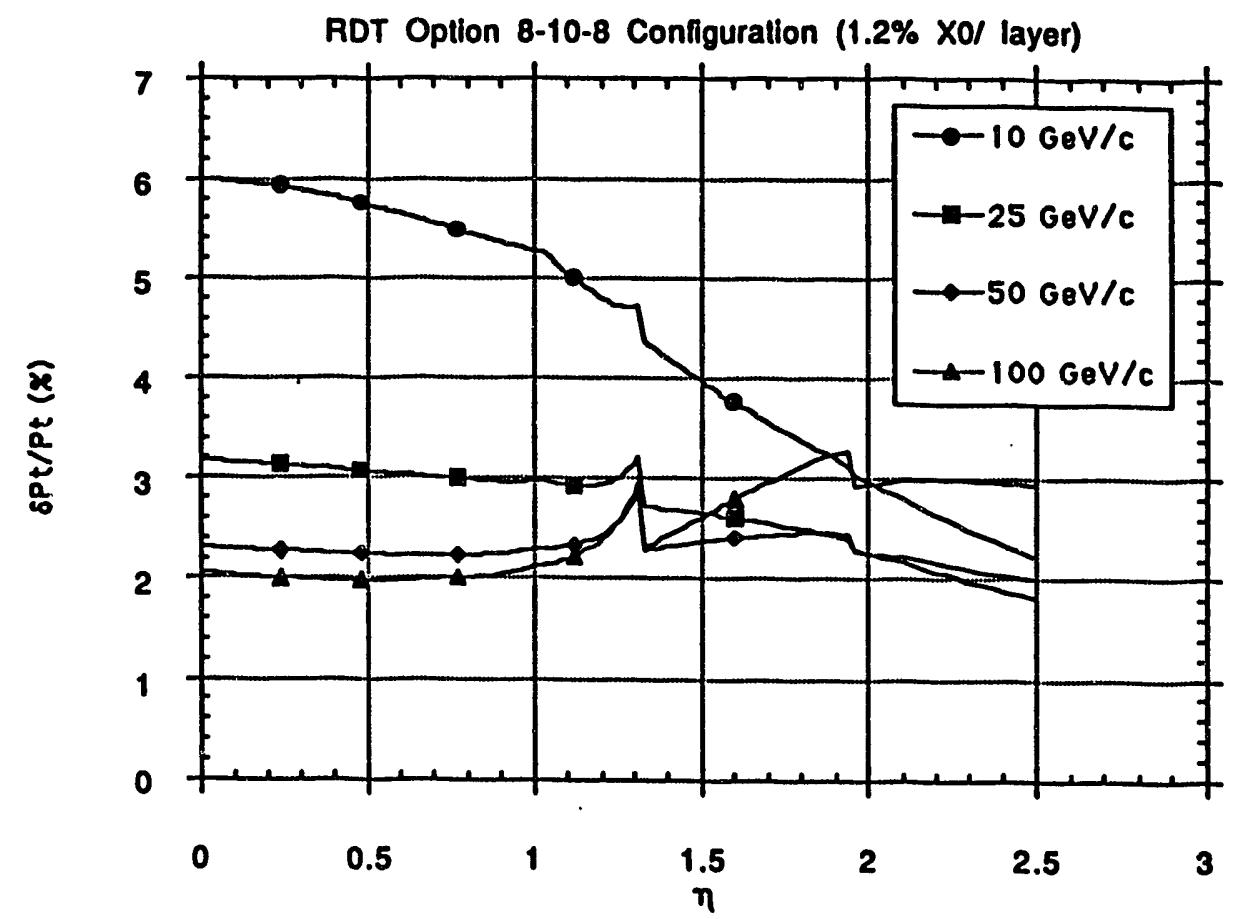

Fig. 8.2.1a

RDT Oplion 8-10-8 Configuration (1.2\% Xonayer)

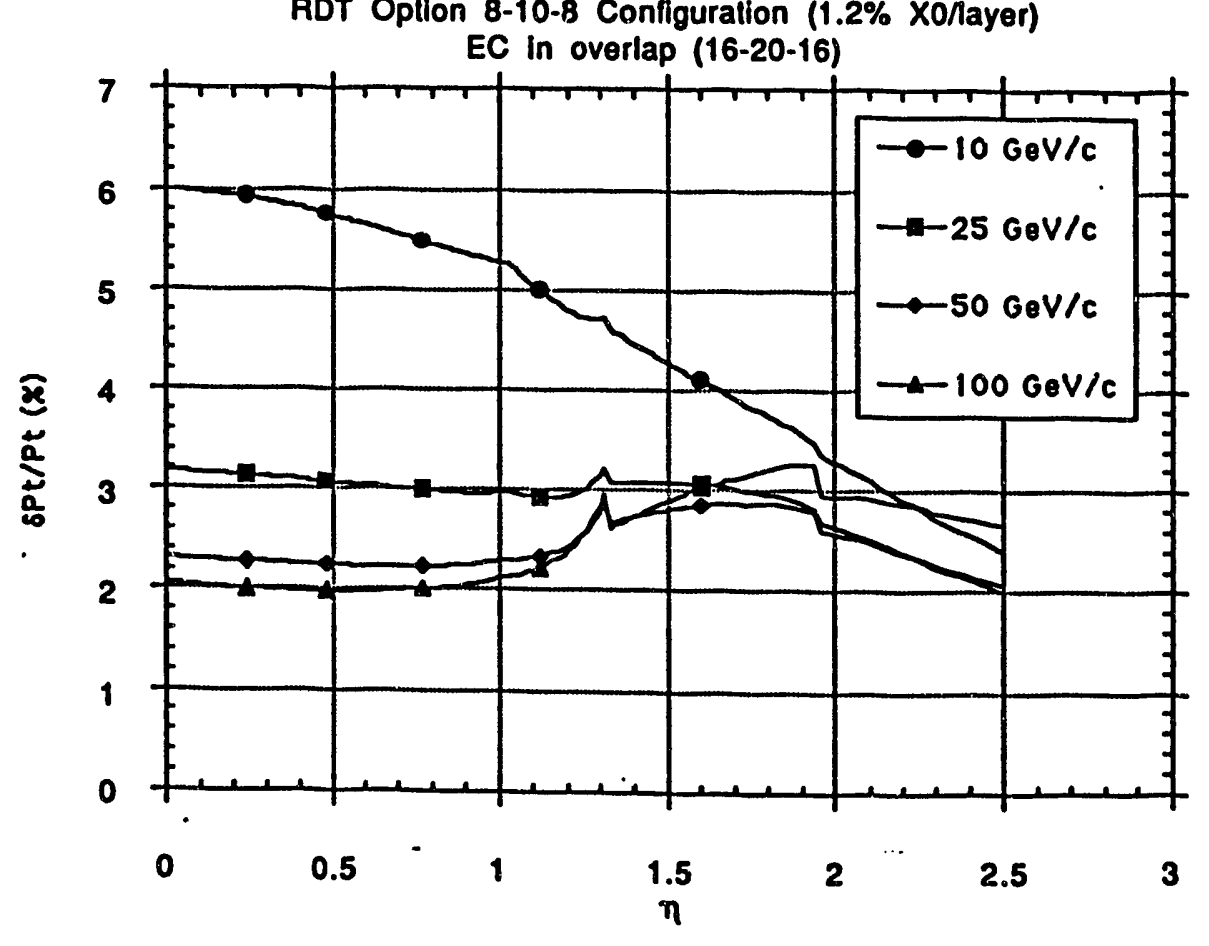




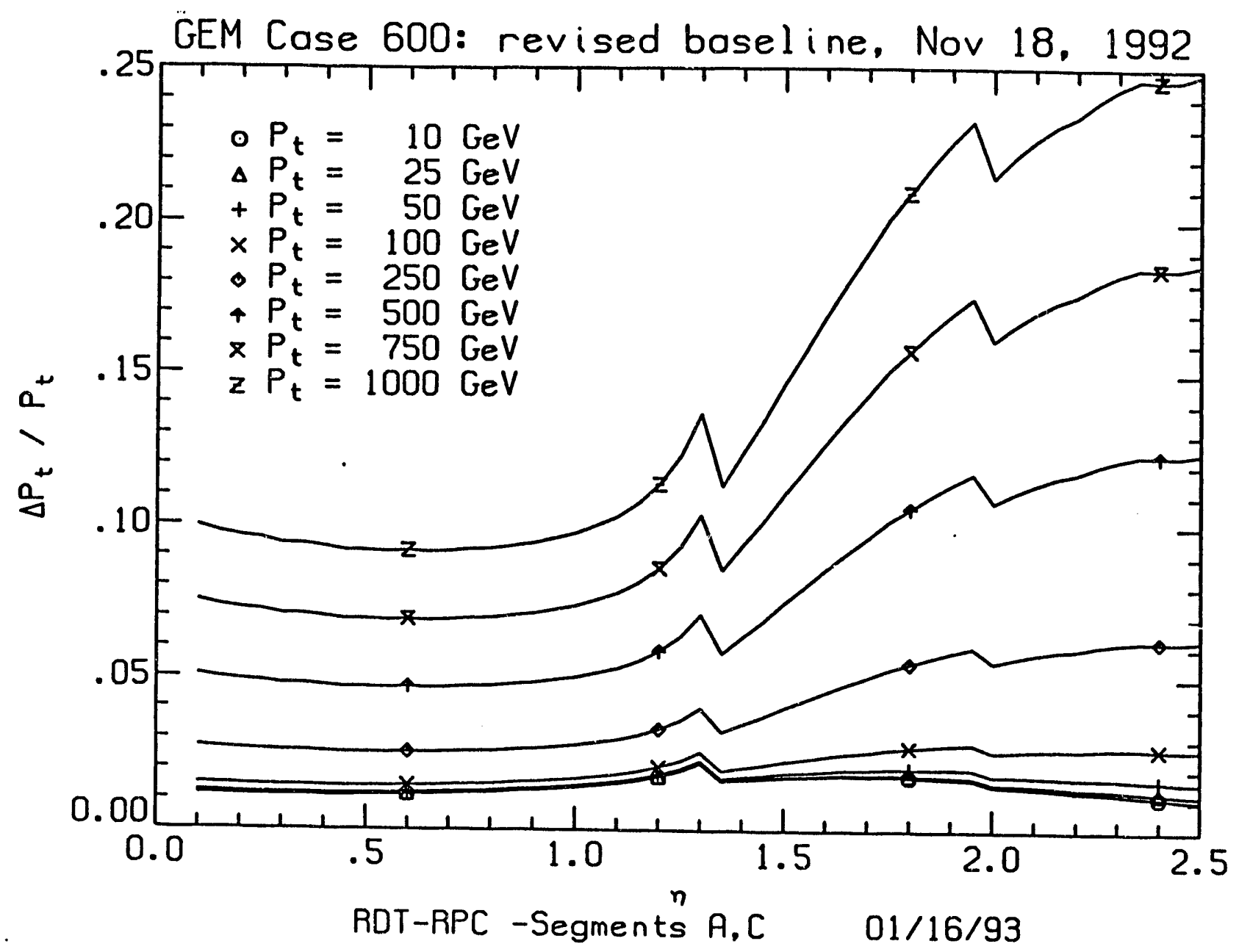

Fig. 8.2.2a

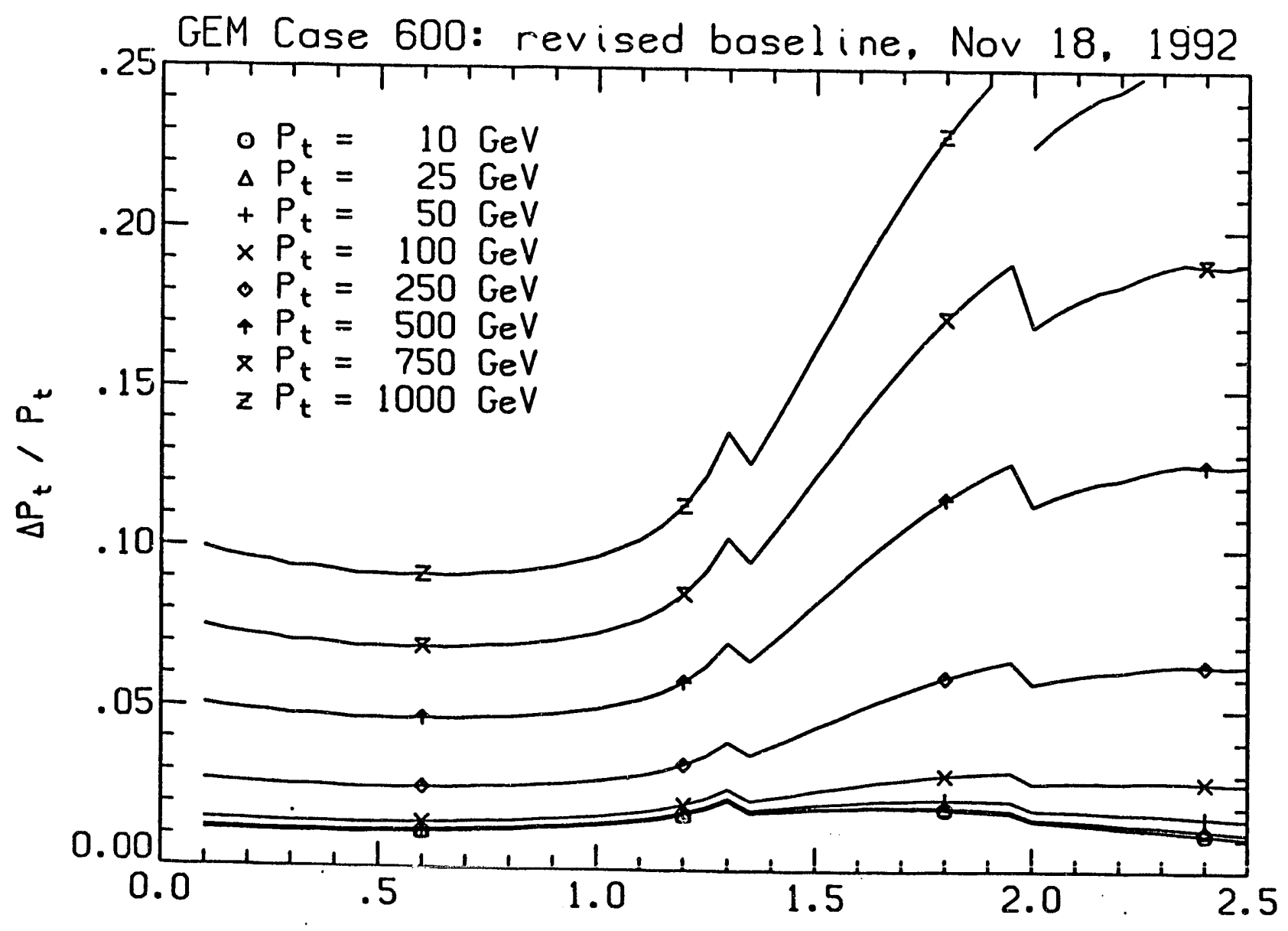

DnT Dor an . . n n 


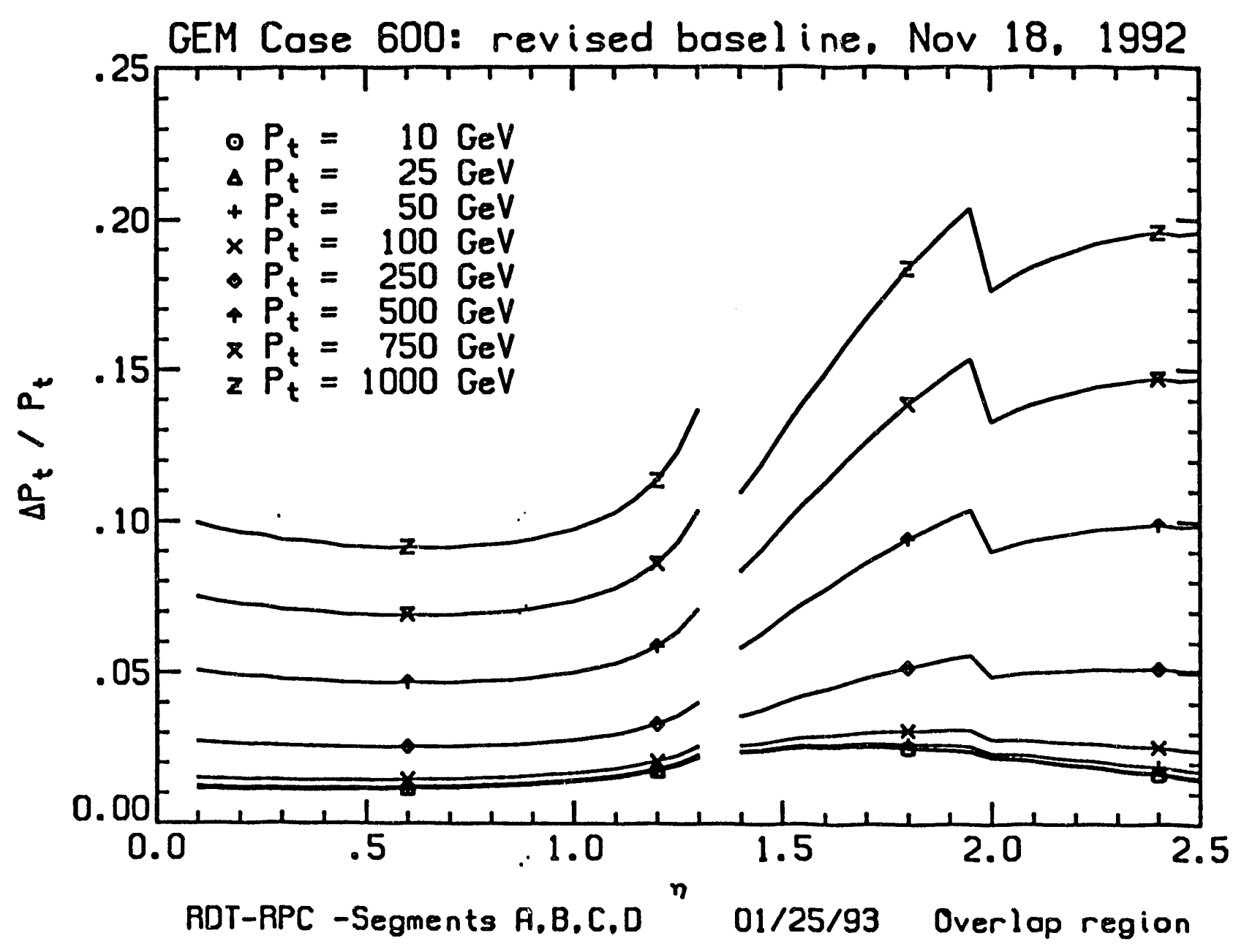

Fig̣. 8.2.2c 

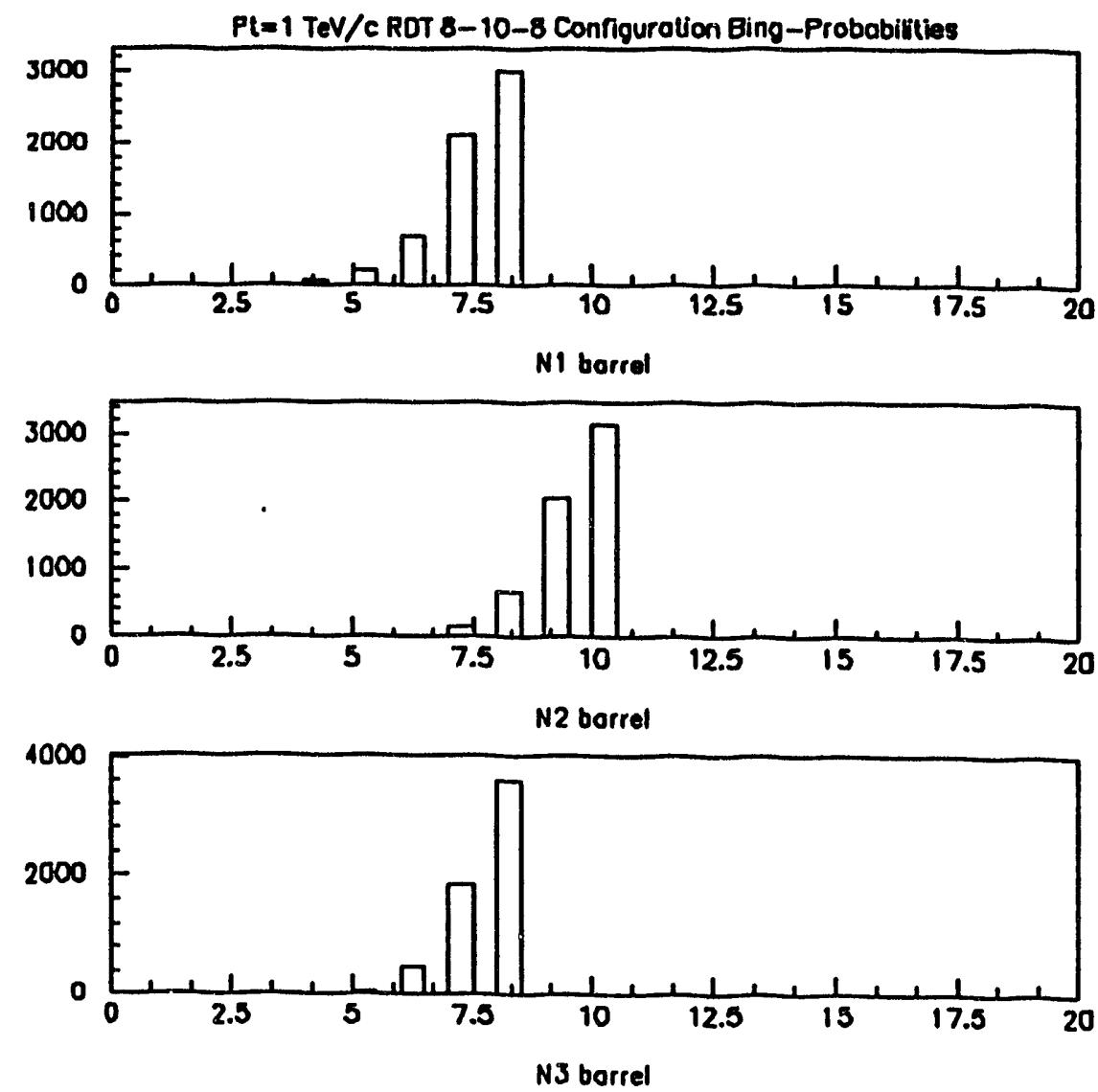

Fig. 8.2.3a
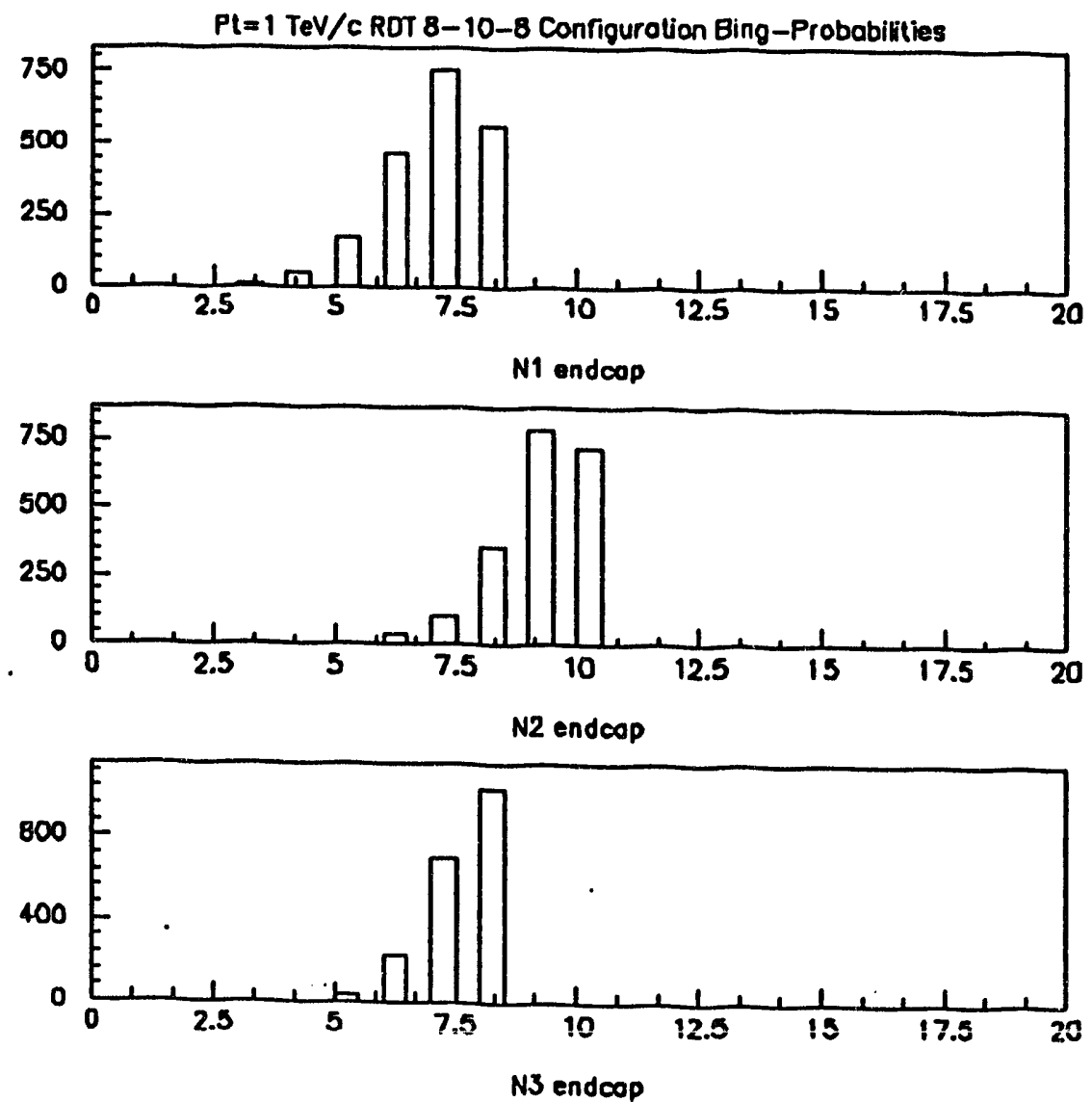

Fig. 8.2.3b 

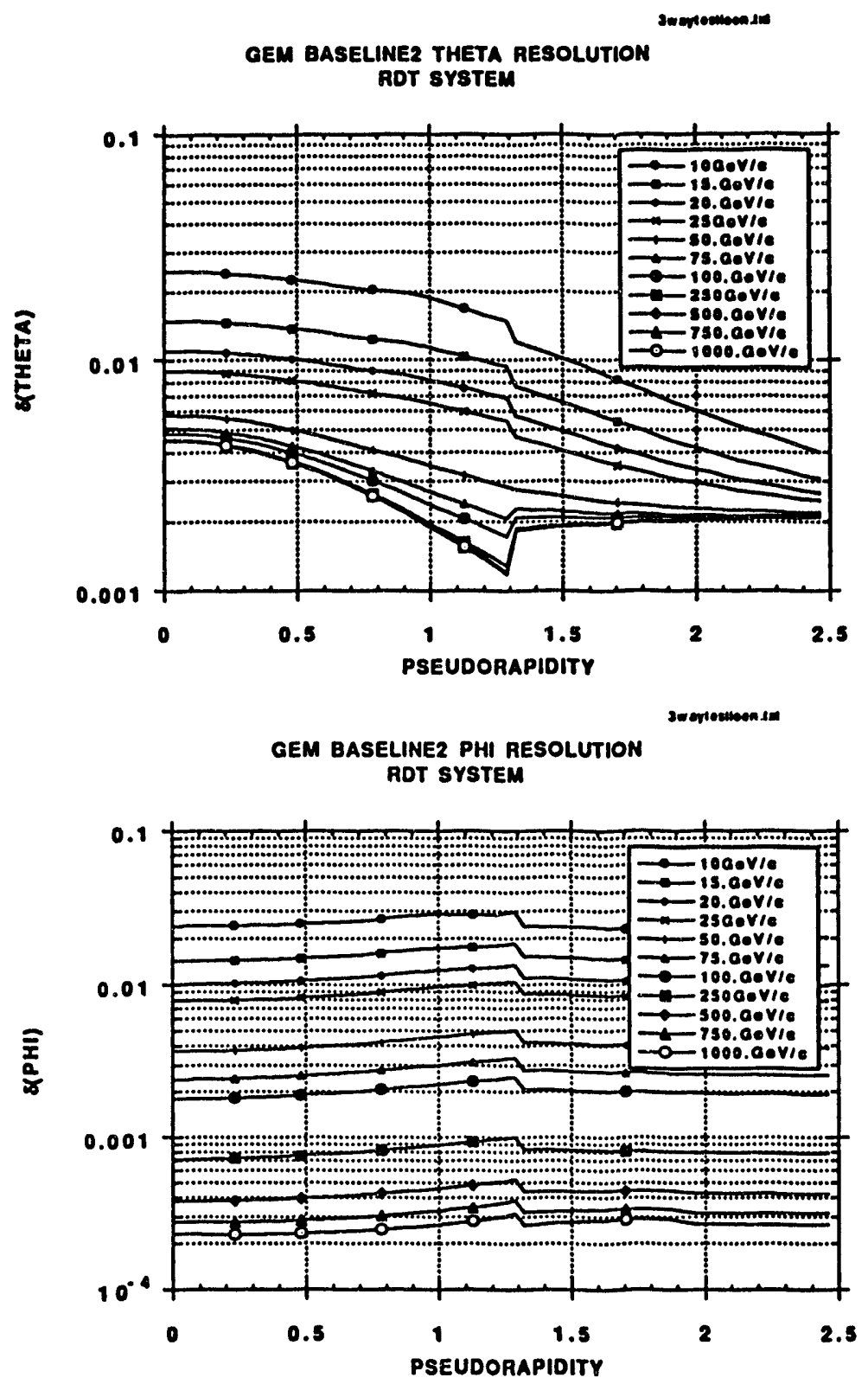

Fig. 8.2.4a

Fig. 8.2.4b

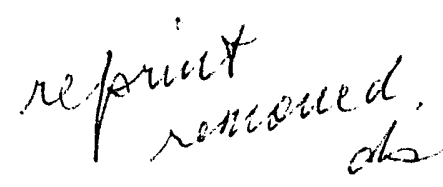




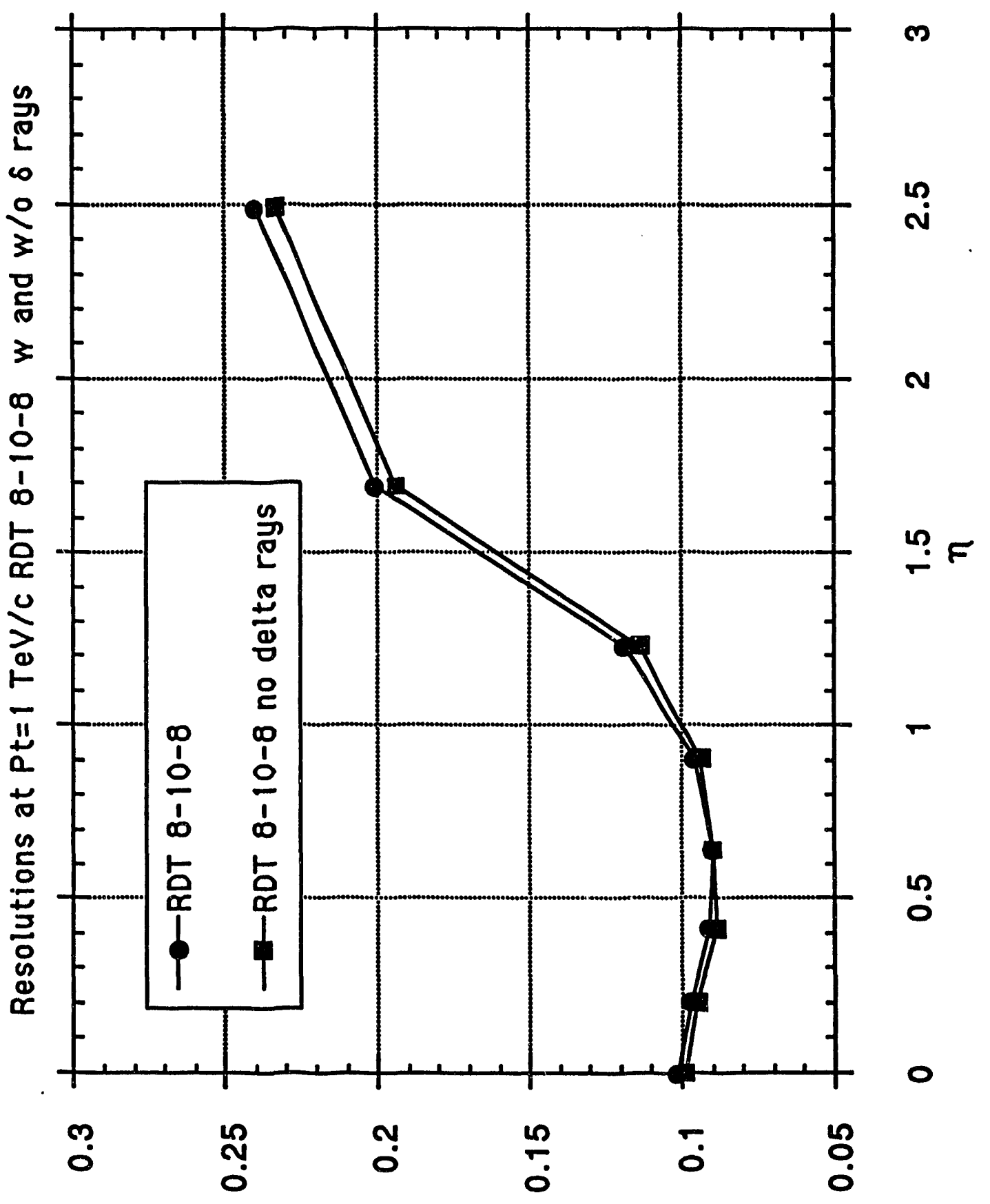

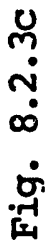




\section{APPENDIX B}

Considerations of strip timing, accidental rates and system dead time.

\section{Barrel Trigger \\ I. Timing}

The basic barrel trigger depends on the non-barrel plane timing. We will label each of the 166 non-bend strips with an index $k(k=0-155)$ where strip $D$ is the strip closest to the interaction point. The non-bend plane strips form a projective tower with the interaction point. Every three corresponding strips (i.e. the three strips with the same index) can be brought into time coincidence at the input to the shift register logic. This is done by having a fixed cable length difference for each strip of SL2 and SL1 with respect to SL3. The fixed cable lengths [measured in nanoseconds] for SL2 and SL1 is listed in Table A.1. Following the cable delay which is common for all strips on a superlayer there is a timing gate delay, the width of which is determined by the bend strip index. Following the gate delays there is generated a signal gate whose length is determined by the superlayer. These timings are also listed in Table A.1.

The bend plane strips also have the same cable differences as the non-bend plane strips as indicated in Table A.1. The bend plane strips do not have any timing gate delays following the cable but rather generate just a signal gate whose width is superlayer dependent. This is also listed in Table A.1.

If the numbers listed in Table A.1 are used in a straight forward, but tedious, hand calculation one finds that all the signals from any track with a transverse momentum greater than $10 \mathrm{GeV}$ which passes through SL1, SL2 and SL3 will arrive in coincidence at the shift register logic with an overlap in time greater than 2 nanoseconds.

It should be noted that this system requires $\pm 5 \%$ logic throughout. This has been included in the above calculations.

\section{A. Accidental Rates}

\section{Uncorrelated Background}

We assume then the neutron flux is $10^{5} \mathrm{Hertz}$ per square centimeter per second everywhere in GEM. Hence our RPC rate is 500 Hertz per square centimeter per second.

We calculate the accidental trigger rate for all tracks with transverse momentum greater than $50 \mathrm{GeV} / \mathrm{c}$. In order to handle this high rate, we place the three RPC 
counters in all super layers in a two out of three coincidence. This yields a 95\% efficient RPC system which can handle the $10^{5}$ neutrons per square centimeter.

The non-bend plane accidental trigger rate $=$ NBA is given by the following formula:

$$
\begin{aligned}
N B A= & {[2]\left[R_{1} R_{2} R_{3}\left(t_{1}+t_{2}\right)\left(t_{1}+t_{3}\right)\right][166][32] } \\
R_{1}= & \text { Rate in superlayer } 1 \\
R_{2}= & \text { Rate in superlayer } 2 \\
R_{3}= & \text { Rate in superlayer } 3 \\
t_{1}= & \text { Signal gate width in superlayer } 1=16 \text { nanoseconds } \\
t_{2}= & \text { Signal gate width in superlayer } 2=16 \text { nanoseconds } \\
t_{3}= & \text { Signal gate width in superlayer } 3=16 \text { nanoseconds } \\
R_{1}= & 3\left(R \times W_{1} L_{1}\right)^{2} \times 2 t_{1} \times[2] \\
& R=500 \\
& W_{1}=3.9 \\
& L_{1}=148.5 \\
& t_{1}=4 \times 10^{-9}
\end{aligned}
$$

The factor [2] is because $2 R_{1}$ strips are involved in the trigger. The factor 3 is due to the combinatorics of the two out of three coincidence.

$$
\begin{aligned}
& R_{1}=2 \times 10^{4} \mathrm{Hertz} \\
& R_{2}= 3\left(\mathrm{R} \times W_{2} \mathrm{~L}_{2}\right)^{2} \times 2 \mathrm{t}_{2} \\
& R=500 \\
& W_{2}=6.5 \\
& L_{2}=239 \\
& t_{2}=12 \times 10^{-9} \\
& t_{3}=16 \times 10^{-9} \\
& R_{2}= 4.3 \times 10^{5} \mathrm{Hertz} \\
& R_{3}= 3\left(\mathrm{R} \times \mathrm{W}_{3} \times \mathrm{L}_{3}\right)^{2} \times 2 \mathrm{t}_{3} \\
& R_{3}= 3(500 \times 8.9 \times 329.6)^{2} \times 2 \times 16 \times 10^{-9}=2.1 \times 10^{6}
\end{aligned}
$$

$\mathrm{NBA}=2 \times 10^{4} \times 4.3 \times 10^{4} \times 21 \times 10^{6} \times 1.6 \times 10^{-8} \times 2.0 \times 10^{-8} \times 166 \times 32=3.2 \times 10^{4}$ Hertz

The non-bend trigger must be placed in coincidence with the bend trigger. Since for every non-bend hit there is a random hit in coincidence in the bend plane, we 
have to multiply NBA by the combinatorial factor $=\mathrm{C}$ to calculate the GEM false trigger rate $=\mathrm{F}$.

$$
\begin{array}{ll}
\mathrm{F}= & \mathrm{NBA} \times \mathrm{C} \\
\mathrm{C}= & \mathrm{N} 3 \times \mathrm{N} 2 \times \mathrm{N} 3
\end{array}
$$

The factor:

N3 $=1$ as one strip in SL3 is hit in coincidence with NBA.

N2 $=3 / 184$ as there are only 3 strips determined by the strip hit in SL3. .h can form a bend plane trigger of either charge that has a transverse momentum of $50 \mathrm{GeV} / \mathrm{c}$.

$\mathrm{N} 1=6 / 115$ as there are only six strips in super layer one that can form a trigger for the hit in SL1.

$$
\begin{array}{ll}
C= & 8.5 \times 10^{-4} \\
F= & 3.2 \times 10^{4} \times 8.5 \times 10^{-4}=27 \text { Hertz }
\end{array}
$$

\section{B. Correlated Background caused by Muons with less than $50 \mathrm{GeV} / \mathrm{c}$ Transverse Momentum}

The trigger rate, in the barrel, for muons with momentum greater than $10 \mathrm{GeV} / \mathrm{c}$ is about 1,000 Hertz. . 'though there is no real calculation, I will assume the number of muon $\mathrm{n}$ the barrel less than $10 \mathrm{GeV} / \mathrm{c}$ is

$6 \times 10^{6}$ Hertz (one low energy muon per 10 crossings), which is of course, essentially the same for muons less than $50 \mathrm{GeV} / \mathrm{c}$. These muons do not make a trigger in the bend plane. The hit they make in :..1 and SL2 in the bend plane is wrong for the hit they make in the bend plane SL3. Hence for this case we must calculate the accidental rate per sector for a $50 \mathrm{GeV} / \mathrm{c}$ bend plane trigger. The accidental rate turns out to be so high that we have to segment the SL1 into three sections along the $Z$ coordinate. Hence each SL1 super layer has a length of $\mathbf{2 1 0 . 6}$ centimeters.

$$
B P A=R 1 R 2 R 3\left(t_{1}+t_{2}\right)\left(t_{1}+t_{3}\right)(254)(32)
$$

We note here that like the non-bend plane counter strips for SL1, SL2 and SL3 are in coincidence.

$$
\begin{array}{ll}
R_{1}=(3)(2)[(210.6)(1.3)(500)]^{2}(6)(16)\left(10^{-9}\right)=1.1 \times 10 \\
R 2= & (3)(2)[(1,042.6)(1.3)(500)]^{2}(3)(32) \times 10^{-9}=26.4 \times 10^{4} \\
R 3= & \text { (3) }(2)[(1.475)(1.3)(500)]^{2}(48)\left(10^{-9}=26.5 \times 10^{4}\right.
\end{array}
$$




$$
\begin{aligned}
& t_{1}=16 \times 10^{-9} \\
& t_{2}=32 \times 10^{-9} \\
& t_{3}=48 \times 10^{-9}
\end{aligned}
$$

$\mathrm{BPA}=(1.1)\left(10^{4}\right)(26.4)\left(10^{4}\right)(26.5)\left(10^{4}\right)(48)\left(10^{-9}\right)(64)\left(10^{-9}\right)(254)=6.0 \times 10^{2}$

This must be put in coincidence with the rate in the non-bend plane per small sector $=$ NBPA.

$$
\mathrm{NBPA}=6 \times 10^{6} / 32 \times 3=6.3 \times 10^{4}
$$

Accidentals per small sector $=$ ASS

$$
\begin{aligned}
\text { ASS } & =(N B P A)(B P A)\left(t_{1}+t_{2}\right) \\
& =(6.3)\left(10^{4}\right)\left(6\left(10^{2}\right)(16+16)\left(10^{-9}\right)=1.2\right.
\end{aligned}
$$

The accidental for the full sector = AFS is given by:

$$
\text { AFS }=(3 \text { ASS })=3.6
$$

Total accidental for GEM $=(3.6)(32)=115$ Hertz. To this we add 27 Hertz which gives the GEM accidental rate of 142 Hertz.

\section{Dead Time}

To calculate the dead time we must consider what is the probability that a track will generate a signal in all three RPC super layers. In each super layer a track goes through three pairs of bend and non-bend strips. Since we use 2 out of 3 logic we must calculate the probability that at least two pairs of bend and non-bend strips are "alive." Let this probability for any bend plane super layer $B_{j}=(L T P)_{B j}, j=1,2$, 3.

$$
\begin{aligned}
& (L T P)_{j}=\left[3 D_{B j}\left(1-D_{B j}\right)^{2}+\left(1-D_{B j}\right)^{3}\right] \\
& D_{B j}=\text { Dead time probability for bend plane strip } B j .
\end{aligned}
$$

Total bend live time $=(\mathrm{LTP})_{\mathrm{B} 1}(\mathrm{LTP})_{\mathrm{B} 2}(\mathrm{LTP})_{\mathrm{B} 3}=\mathrm{L}_{\mathrm{B}}$

Total bend dead time $=1-(\text { LTP })_{1}(\text { LTP })_{2}(\text { LTP })_{3}=D_{B}$ 
We will assume that after each hit on any strip the strip is "dead" for $100 \times 10^{-9}$ seconds. From the previous information we calculate:

$$
\begin{aligned}
& D_{B 1}=(631.9)(1.3)(500)(100)\left(10^{-9}\right)=.041 \\
& D_{B 2}=(1,042.6)(1.3)(500)(100)\left(10^{-9}\right)=.068 \\
& D_{B 3}=(1,475)(1.3)(500)(100)\left(10^{-9}\right)=.096 \\
& D_{N B 1}=(148.5)(3.9)(500)(100)\left(10^{-9}\right)=.028 \\
& D_{N B 2}=(239)(6.5)(500)(100)\left(10^{-9}\right)=.077 \\
& D_{N B 3}=(329)(8.9)(500)(100)\left(10^{-9}\right)=.147 \\
& D_{N B j} j=1,2,3 \text { is the dead time probability for non-bend plane strip } B_{j} \\
& (L T P)_{B 1}=(3)(.04)(.96)^{2}+(.96)^{3}=.995 \\
& (L T P)_{B 2}=(3)(.068) .(.932)^{2}+(.932)^{3}=.987 \\
& (L T P)_{B 3}=(3)(.096)(.904)^{2}+(.904)^{3}=.974
\end{aligned}
$$

Total bend live time $=\mathrm{LB}=(.995)(.987)(.974)=.957$

Total bend dead time $=1-\mathrm{LB}=1-.957=.043$

Let $(\mathrm{LTP})_{\mathrm{NBj}}=$ Live time probability of the non-bend plane $j$.

$$
\begin{aligned}
& (\mathrm{LTP})_{\mathrm{NB} 1}=(3)(.028)(.972)^{2}+(.972)^{3}=.998 \\
& (\mathrm{LTP})_{\mathrm{NB} 2}=(3)(.077)(.923)^{2}+(.923)^{3}=.983 \\
& (\mathrm{LTP})_{\mathrm{NB3}}=(3)(.147)(.853)^{2}+(.853)^{3}=.942
\end{aligned}
$$

Let $\mathrm{LNB}=$ total non-bend live time.

$$
\mathrm{LNB}=(\mathrm{LTP})_{\mathrm{NB} 1}(\mathrm{LTP})_{\mathrm{NB} 2}(\mathrm{LTP})_{\mathrm{NB}}
$$

Total non-bend dead time $=1-$ LNB

$$
\text { LNB }=(.998)(.983)(.942)=.924
$$


Total non-bend dead time $=1-.924=.076$

Let total GEM live time $=\mathrm{LT}$

$$
\mathrm{LT}=(\mathrm{LB})(\mathrm{LNB})=(.957)(.924)=.88=88 \%
$$

GEM dead time $=12 \%$

If we assume our pulse dead time is 50 nanoseconds instead of 100 the dead time is reduced to about $6 \%$. Both numbers are acceptable. If we assume, because of the high rates, the efficiency of the RPC degrades then there will be a different calculation for the GEM live time and dead time. Assume that the efficiency of an RPC plane is $E$.

\begin{tabular}{|c|c|c|}
\hline E & GEM Live Time & GEM Dead Time \\
\hline $95 \%$ & $96 \%$ & $4 \%$ \\
\hline $90 \%$ & $84 \%$ & $16 \%$ \\
\hline $85 \%$ & $69 \%$ & $31 \%$ \\
\hline $80 \%$ & $52 \%$ & $48 \%$ \\
\hline
\end{tabular}

Obviously the dead time is sensitive to the efficiency of the individual RPC plane. A proper, by definition, high rate RPC will have an efficiency greater then $95 \%$. It should be noted that at efficiencies greater than $95 \%$, the previous calculation is the relevant calculation.

\section{End Cap}

The problems of the end cap are much simpler than the barrel due to the much greater segmentation. Without presenting the details, all accidentals and dead times are reduced by about two orders of magnitude. The timings are essentially as in the barrel, but all delays are reduced by about a factor two. 


\section{TABLE B.1}

Cable length difference for all strips (both bend plane and non-bend plane):

$\begin{array}{ll}\text { SL3 and SL2 } & =30.65 \text { (nanoseconds) } \\ \text { SL3 } 3 \text { and SL1 } & =59.82 \text { (nanoseconds) }\end{array}$

(Note about half this (difference) is used just to get the signals to the input to the shift registers). This cable delay should be accurate to \pm 0.5 nanoseconds or about \pm 10 centimeters of cable.

The delay gate width $\mathrm{DT}_{k}$ for non-bend strip $\mathrm{k}$ superlayer SL3:

$$
\mathrm{DT}_{\mathrm{k}}=(\mathrm{k} / 165)(31) \text { nanoseconds }(\mathrm{k}=0 \text { to } 165)
$$

\section{Superlayer SL2:}

$$
\mathrm{DT}_{\mathrm{k}}=(\mathrm{k} / 165)(13) \text { nanoseconds }(\mathrm{k}=0 \text { to } 165)
$$

$$
\begin{aligned}
& \text { Superlayer SLI: } \\
& \mathrm{DT}_{\mathrm{k}}=0
\end{aligned}
$$

The error on any delay gate width can be as late as $\pm 5 \%$.

The signal gate width $\mathrm{DT}_{\mathbf{S}}$ for non-bend strips:

Superlayer SL3:

$\mathrm{DT}_{\mathrm{S}}=11.5$ nanoseconds

Superlayer SL2:

$\mathrm{DT}_{\mathrm{S}}=10$ nanoseconds

Superlayer SL1:

$\mathrm{DT}_{\mathrm{S}}=4$ nanoseconds

The signal gate width $\mathrm{DT}_{\mathbf{S}}$ for bend strips superlayer SL3:

$\mathrm{DT}_{S}=42.5$ nanoseconds

Superlayer SL2:

$\mathrm{DT}_{\mathrm{S}}=23$ nanoseconds

Superlayer SL1:

$\mathrm{DT}_{\mathrm{S}}=4$ nanoseconds

The error on any signal gate width can be as large as $\pm 5 \%$. 
The accidental rate turns out to be so high that we have to segment the SL1 into three sections along the $\mathbf{Z}$ coordinate. Hence each SLI super layer has a length of 210.6 centimeters.

$$
R_{1}=(3)(2)[(210.6)(1.3)(500)]^{2}(6)(16)\left(10^{-9}\right)=1.1 \times 10^{4}
$$

$\mathrm{BPA}=(1.1)\left(10^{4}\right)(26.4)\left(10^{4}\right)(26.5)\left(10^{4}\right)(48)\left(10^{-9}\right)(64)\left(10^{-9}\right)(254)=6.0 \times 10^{2}$

This must be put in coincidence with the rate in the non-bend plane per small sector $=$ NBPA.

$$
\mathrm{NBPA}=6 \times 10^{6} / 32 \times 3=6.3 \times 10^{4}
$$

Accidentals per small sector $=$ ASS

$$
\text { ASS }=(N B P A)(B P A)\left(t_{1}+t_{2}\right)=(6.3)\left(10^{4}\right)\left(6\left(10^{2}\right)(16+16)\left(10^{-9}\right)=1.2\right.
$$

The accidental for the full sector = AFS is given by:

$$
\text { AFS }=(3 \text { ASS })=3.6
$$

Total accidental for GEM $=(3.6)(32)=115$ Hertz. To this we add 27 Hertz which gives the GEM accidental rate of 142 Hertz. 


\section{Appendix C}

\section{Cost of Baseline - RDT/RPC System}

RDT: $8-10-8$ configuration in both the barrel and endcaps ( 348,000 drift channels) RPC: 3-3-3 configuration

\begin{tabular}{|l|l|l|l|}
\hline Item & Barrel & Endcaps & Total \\
\hline Sector assembly & 4835 & 3905 & 8741 \\
\hline RDT Chambers & 21191 & 13596 & 34787 \\
\hline RPC Chambers & 6595 & 4579 & 11174 \\
\hline Support Structure & 3216 & 2415 & 5630 \\
\hline Gas Systems & 3501 & 3355 & 6856 \\
\hline Cooling Systems & 143 & 150 & 292 \\
\hline Alignment & 2192 & 809 & 3001 \\
\hline Installation & 2890 & 1475 & 4364 \\
\hline Mechanical Subtotal & 37968 & 25704 & 74845 \\
\hline $\begin{array}{l}\text { RDT Electronics } \\
\text { Subtotal }\end{array}$ & 5607 & 9818 & 15425 \\
\hline $\begin{array}{l}\text { RPC Electronics } \\
\text { Subtotal }\end{array}$ & 1300 & 1300 & 2600 \\
\hline Subtotal & 6907 & 11118 & 18025 \\
\hline R\&D & 2423 & 2246 & 4669 \\
\hline Conceptual design & & & 1692 \\
\hline Project Mazadgement & & & 3731 \\
\hline Subtota & & & 10092 \\
\hline $\begin{array}{l}\text { Total } \\
\text { (w/o contingency) }\end{array}$ & & & 102962 \\
\hline Contingency (28\%) & & & 28829 \\
\hline Grand total & & 131791 \\
\hline
\end{tabular}

Notes:

(a) The RDT electronics were computed at Costs $=\$ 2.1 \mathrm{M}+\$ 38.29 \times$ channels (Dan Marlow).

(b) The RPC electronics costs were doubled to include frontend electonics and boards mounted on chambers.

Source of information: Rick Sawicki and Dick Gustavson 1/17/93. 


\section{Cost of Baseline - RDT/PWC System}

RDT: $8-10-8$ configuration in both the barrel and endcaps ( 348,000 drift channels) PWC: 4-3-3 configuration - scaled from CSC barrel chamber costs (see below).

\begin{tabular}{|l|l|l|l|}
\hline \hline Item & Barrel & Endcaps & Total \\
\hline Sector assembly & 4835 & 3905 & 8741 \\
\hline RDT Chambers & 21191 & 13596 & 34787 \\
\hline PWC Chambers & 6000 & 2000 & 8000 \\
\hline Support Structure & 3216 & 2415 & 5630 \\
\hline Gas Systems & 3501 & 3355 & 6856 \\
\hline Cooling Systems & 143 & 150 & 292 \\
\hline Alignment & 2192 & 809 & 3001 \\
\hline Installation & 2890 & 1475 & 4364 \\
\hline Mechanical Subtotal & & & 71671 \\
\hline $\begin{array}{l}\text { RDT Electronics } \\
\text { Subtotal }\end{array}$ & 5607 & 9818 & 15425 \\
\hline $\begin{array}{l}\text { PWC Electronics } \\
\text { Subtotal }\end{array}$ & 1300 & 1300 & 2600 \\
\hline Subtotal & 6907 & 11118 & 18025 \\
\hline R\&D & 2423 & 2246 & 4669 \\
\hline Conceptual design & & & 1692 \\
\hline Project Management & & & 3731 \\
\hline Subtotal & & & 10092 \\
\hline $\begin{array}{l}\text { Total } \\
\text { (w/o contingency) }\end{array}$ & & & 99788 \\
\hline Contingency (28\%) & & & 27941 \\
\hline Grand total & & & 127729 \\
\hline
\end{tabular}

Notes:

(a) The RDT electronics were computed at Costs $=\$ 2.1 \mathrm{M}+\$ 38.29 \times$ channels.

(b) The PWC electronics costs taken to be same as RPC costs above.

(c) The PWC costs were determined from the CSC barrel chamber costs by the following scaling:

- reduce cost of precision strip panels by 0.10

- reduce cost of hexcell panels by 0.10

- fraction of CSC cost in above two items is $25 \%$ and $61 \%$, respectively

- cost of 6-6-4 CSC barrel chambers $\$ 41 \mathrm{M}$

- area of 4-3-3 PWC system for GEM is $11785.6 \mathrm{~m}^{2}$

- cost of PWC system so scaled $\$ 679 / \mathrm{m}^{2}$

Source of information: Rick Sawicki and Dick Gustavson 1/17/93. 


\section{Appendix D}

\section{RDT System with a PWC - To Trigger}

The prompt nature of a PWC signal is the main advantage of the CSC proposal while the natural compensation for ExB effects and lower costs are the RDT strong points. We combine both with a proposal to use two layers of PWC (in a wire-OR) at each superlayer and use a coincidence of the three superlayers to measure the time of the muon crossing. The time of the crossing is then combined with the momentum determined from the RDT hits to form the muon trigger. It should be noted that the time of the muon crossing is needed only for muons which are in muon triggers, i.e., for high momentum tracks. In all other triggers the time of the muon crossing can be determined off-line from the RDT data.

The PWC wires are laid on a $2.5 \mathrm{~mm}$ pitch in a direction perpendicular to the drift tubes with projective staggering at each superlayer. They are grouped into $\Delta \eta=.025$, $\Delta \phi=0.2$ trigger elements ( total) with a maximum length of $1.6 \mathrm{~m}( \pm 4 \mathrm{~ns})$. In superlayer- 2 the segments are $15 \mathrm{~cm}$ wide at $\eta=0$ decreasing to $5.0 \mathrm{~cm}$ wide at $\eta$ $=2.0$. In the high muon-flux region $\eta=2.0-2.5$ the orientation of the PWC wires is changed to parallel to the drift. tubes with $\Delta \eta=0.25, \Delta \phi=.02$ trigger elements (3768 total). The to pre-trigger is a coincidence of 1 hodoscope element in each of the three superlayers in a straight line pointing toward the collision point. The coincidence is strobed with the accelerator clock and the earliest time in the coincidence is the to for the muon track at that $h$.

A neutron fluxxefficiency of $100 \mathrm{~Hz} / \mathrm{cm}^{2}$ yields a maximum signals rate of about $0.6 \mathrm{MHz}$ in the largest hodoscope elements (near $\eta=0$ ). Assuming a 20 ns wide signal the triple-coincidence probability is $8 \times 10^{-6}$; the coincidence rate from neutrons is then $<5 \mathrm{~Hz}$. The coincidence rate from tracks near $\eta=0$ is very low due to the filtering of the calorimeter. Near $\eta=2.5$ the energy spectrum is much harder and the rate can be as high as $25 \mathrm{kHz}$ in a hodoscope element and also in the three-element coincidence. Tracks in this region separated by more than $100 \mathrm{nsec}$ (>99\%) will have a unique to association with the drift information.

Since this proposal uses a PWC trigger, it has many similarities to the CSC proposal, however, the momentum information is obtained from the drift tubes alone while only to need be obtained from the PWC planes. Consequently, only a small number of PWC channels are needed at each superlayer that yield a total channel count of Since the PWCs have continuous cathodes that are easy to miake and have easily achievable tolerance requirements, an estimate of the PWC cost is not more than $1 \mathrm{k} \$ / \mathrm{m}^{2}$. In the system proposed there are about $3500 \mathrm{~m}^{2}$ of PWC and thus the cost will be $3.5 \mathrm{M} \$$ with contingency added the total price tag will be arourd $5 \mathrm{M} \$$. 


\section{RDT/PWC Trigger and Layout}

Drift Tube Sections for DA and Momentum Trigger

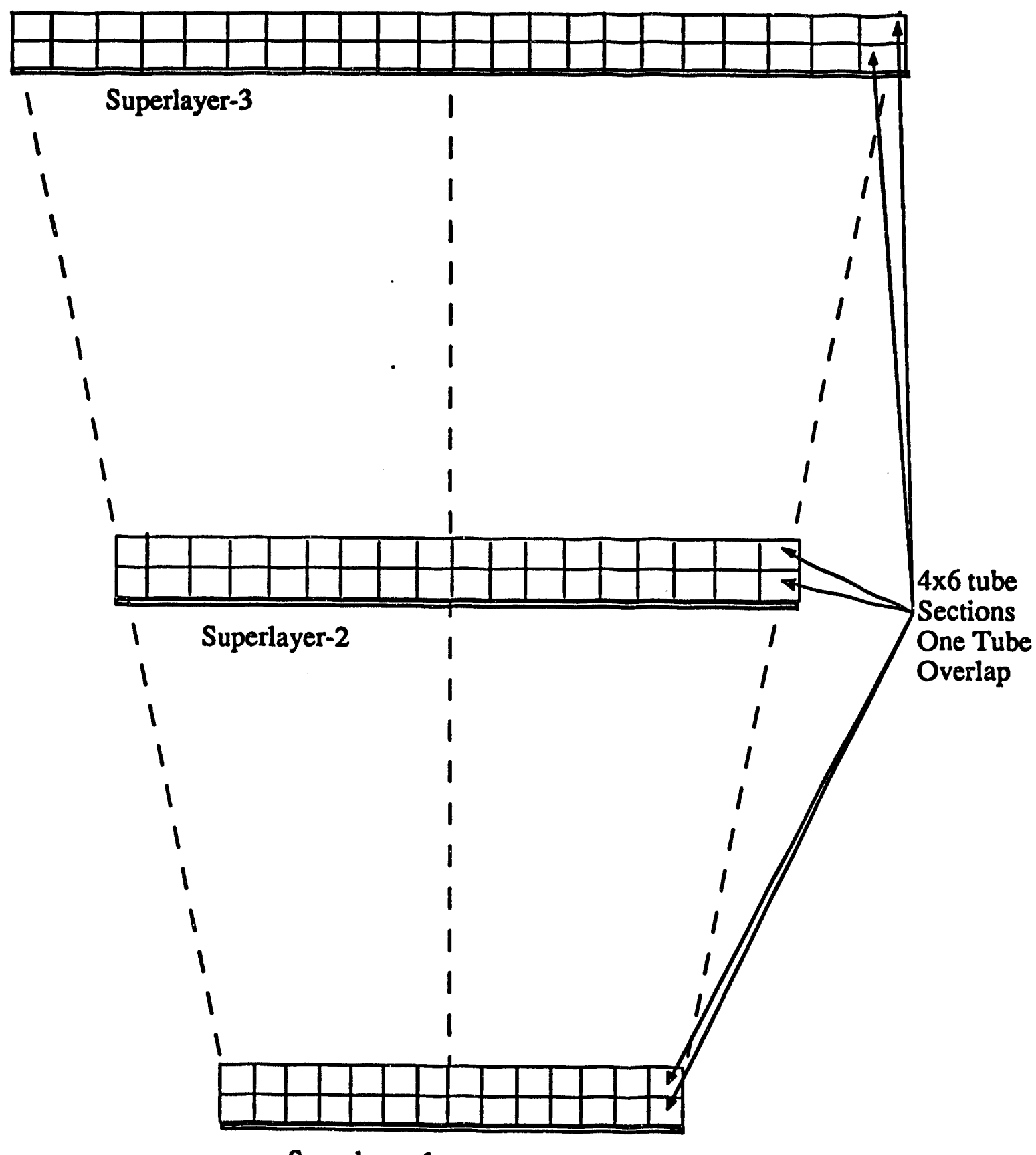

Superlayer-1 
Muon Pt Trigger Components
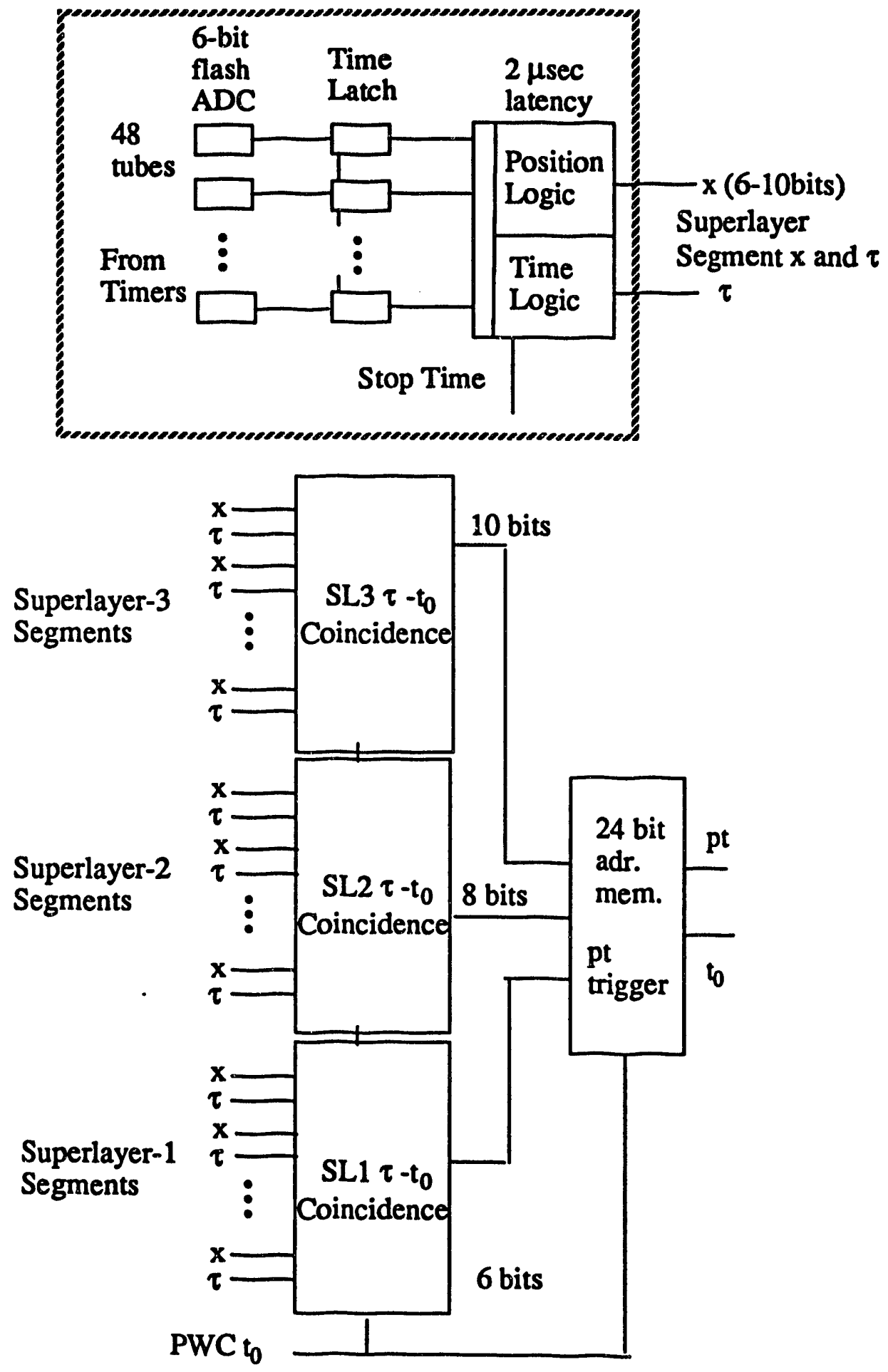
PWC segmentation in $\eta$ and $\phi$

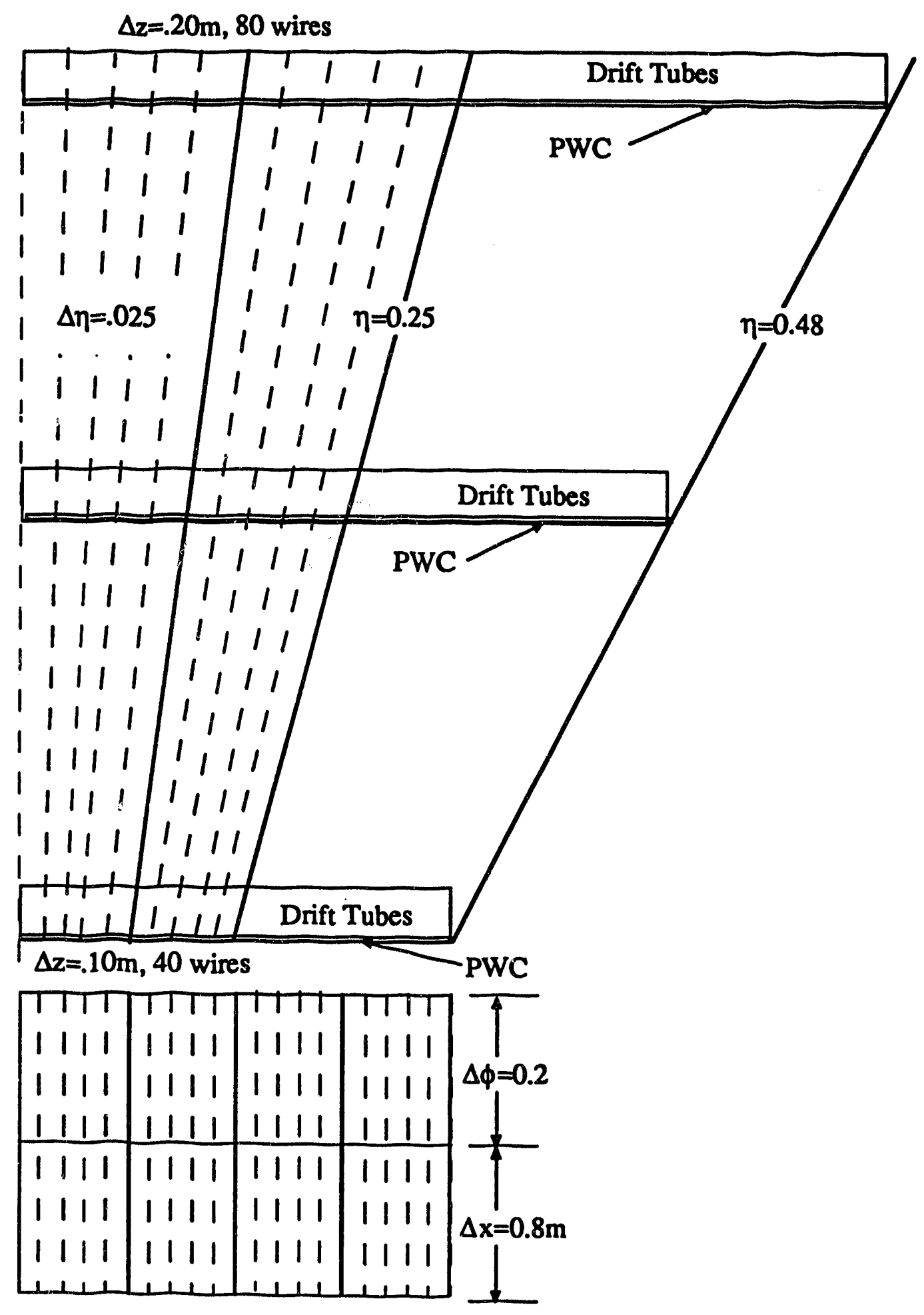


Track-Segment Pattern Logic

(Max. Effective Rates)

(single-double buffered timing)

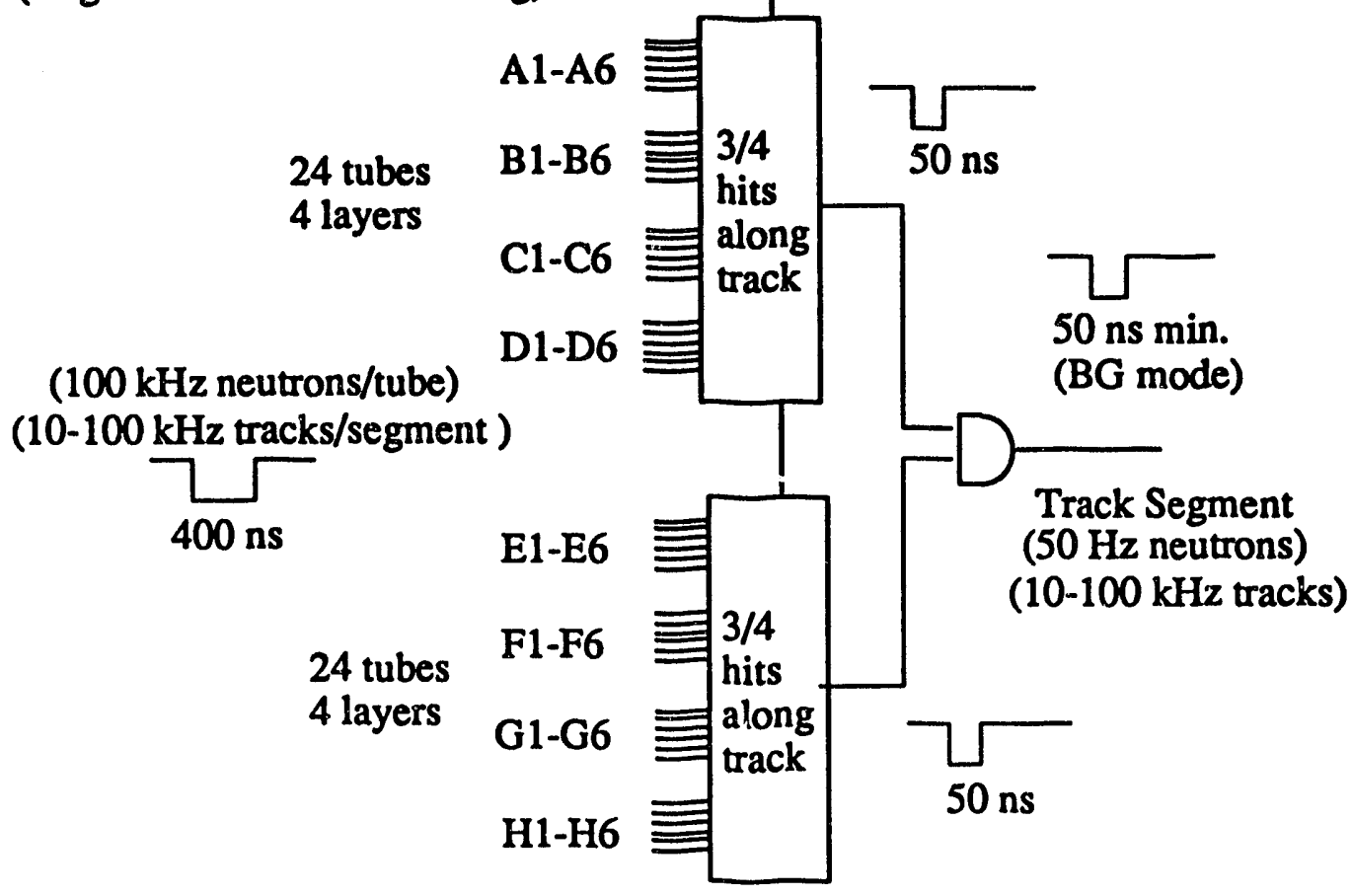

Track Segment Drift Timer

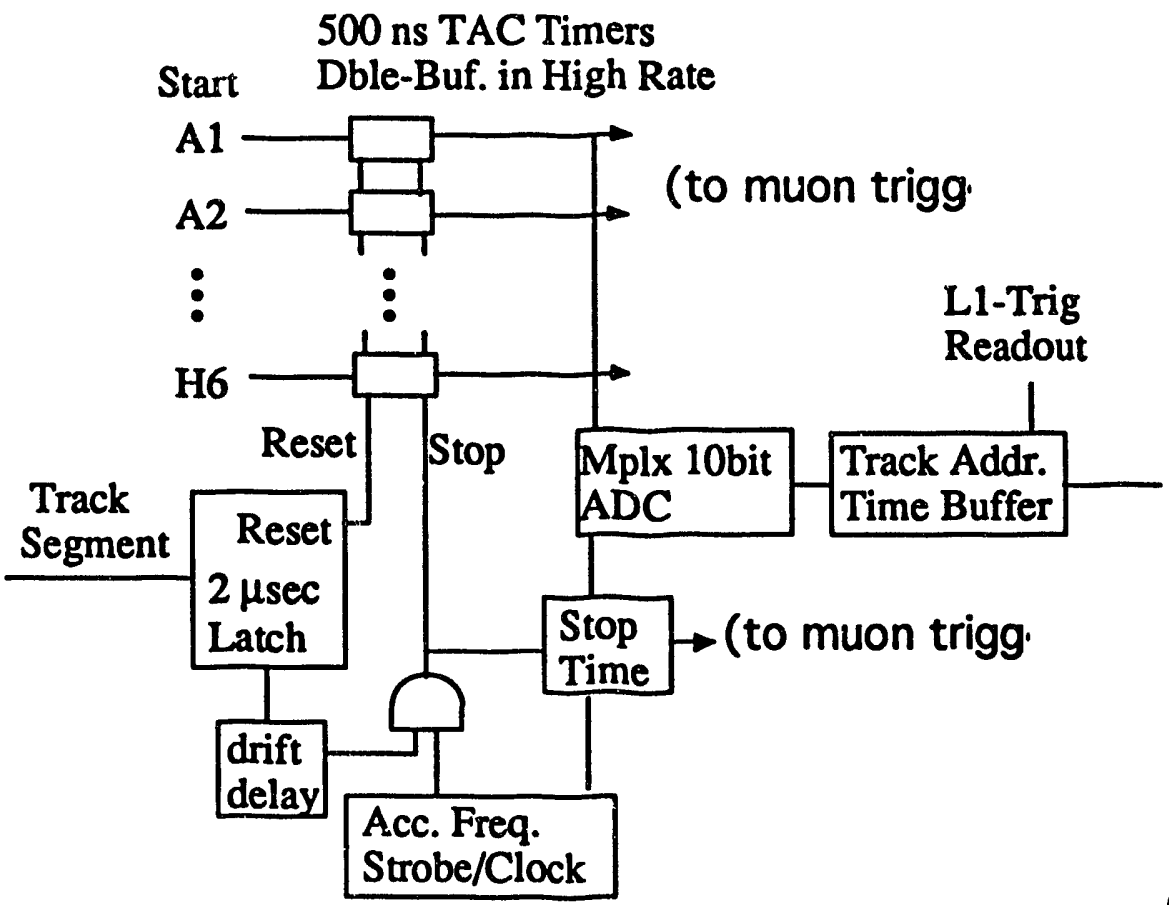

Acc. Freq. $20 \mathrm{MHz}$ Strobe 

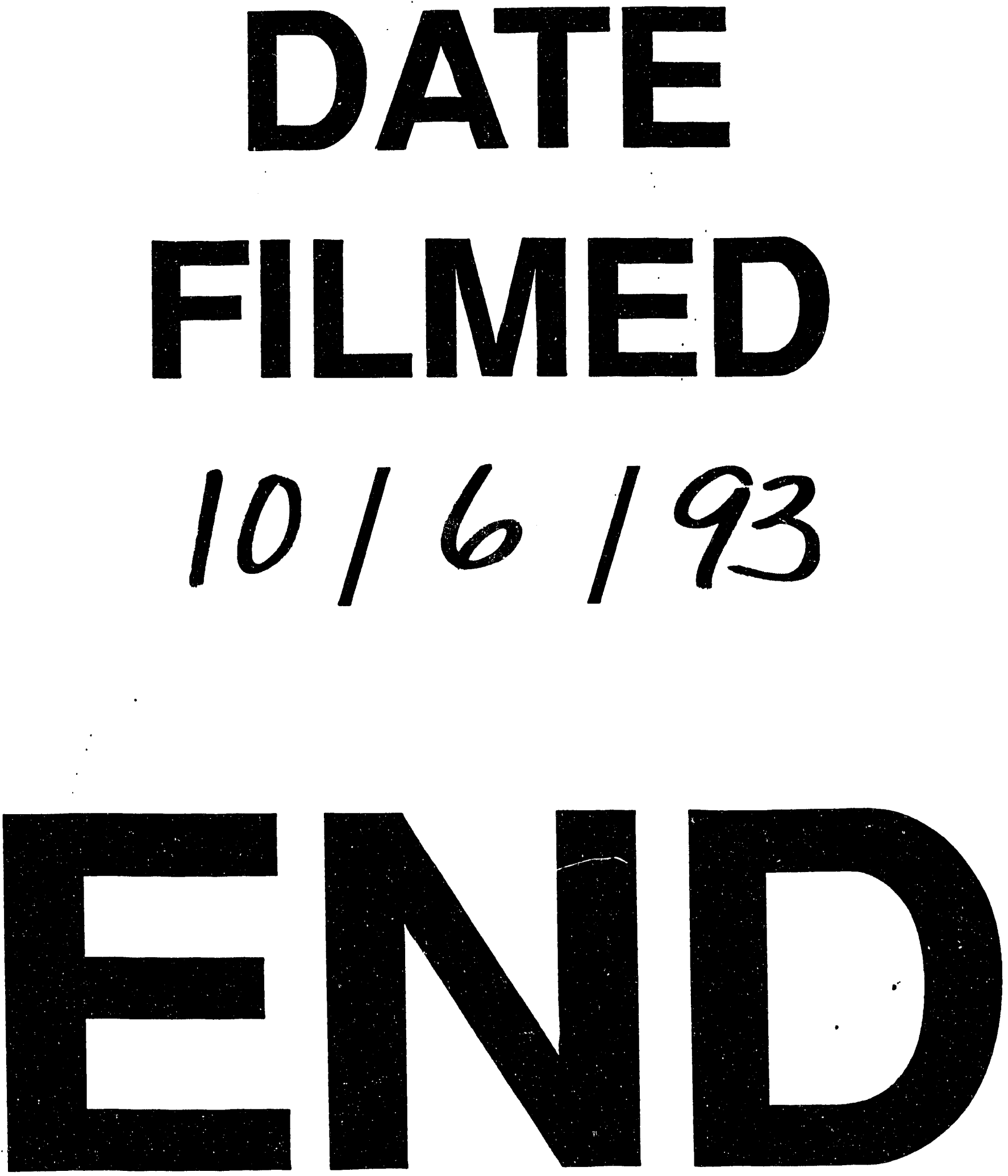